

\section{ON A SLIDING FRICTION MODEL OF BOUNDARY LUBRICATION}

Can Wang 


\section{Graduation Committee:}

Chair / secretary:

prof.dr.ir. H.F.J.M. Koopman

University of Twente

Supervisor:

prof.dr.ir. D.J. Schipper

University of Twente

Committee Members: prof. dr. ing. A. Fischer

University of Duisburg-Essen

prof. dr. J. Jamari

University of Diponegoro

prof.dr. G.J. Vancso

University of Twente

prof.dr.ir. J.E. ten Elshof

University of Twente 


\title{
ON A SLIDING FRICTION MODEL OF BOUNDARY LUBRICATION
}

\section{DISSERTATION}

\author{
to obtain \\ the degree of doctor at the Universiteit Twente, \\ on the authority of the rector magnificus, \\ prof. dr. ir. A. Veldkamp, \\ on account of the decision of the Doctorate Board \\ to be publicly defended \\ on Thursday 23 September 2021 at 14.45 hours
}

by

\section{Can Wang}

born on the 26th of July, 1990

in Anhui, China 
This dissertation has been approved by:

Supervisor

prof. dr. ir. D.J. Schipper

This work was financially supported by Bosch Transmission Technology B.V. under the project number 20918349.

ISBN: 978-90-365-5251-6

DOI: $10.3990 / 1.9789036552516$

(C) 2021 Can Wang, The Netherlands. All rights reserved. No parts of this thesis may be reproduced, stored in a retrieval system or transmitted in any form or by any means without permission of the author. Alle rechten voorbehouden. Niets uit deze uitgave mag worden vermenigvuldigd, in enige vorm of op enige wijze, zonder voorafgaande schriftelijke toestemming van de auteur. 


\section{Summary}

When tribological contacts operate under boundary lubrication, lubricant films are not thick enough to fully separate the two surfaces. In these cases, surface to surface contacts occur, while asperity interactions and surface adhesion dominate the friction. In this thesis, a boundary lubrication friction model will be described with these two mechanisms considered. Both numerical analysis and experimental works were employed to build and validate the model.

To characterize the tribofilms formed during boundary lubrication, the thickness and chemical composition of the tribofilms were firstly quantified by AFM, FIB-SAM and XPS. Afterwards, nano-indentations were performed on the tribofilms to determine their mechanical properties.

A numerical model based on the semi-analytical method was developed to calculate the tangential forces caused by asperity interactions. Elasto-plastic deformations of both tribofilm and substrate were considered. The effects of tribofilm thickness, interference depth and mechanical properties of tribofilm on friction were investigated.

The sensitivity of SAM calculating real contact areas is dealt with in this thesis. The real contact areas of a sinusoidal wavy surface on a flat were calculated by both SAM and an analytical method. The results were compared and an extrapolation was proposed to acquire a better estimation of the real contact area calculated by SAM.

Finally, the effect of adhesive forces and asperity interactions on friction were combined and the total frictions were calculated. The results were validated by pinon-disc test results.

The thesis consists of two parts. The first part (part A) summarizes the work in six chapters. The second part (part B) lists the journal articles in which the details are described. 


\section{Samenvatting}

Wanneer tribologische contacten werken onder condities van grenssmering dan zijn de smeerfilmdiktes te klein om de oppervlakken volledig te scheiden. In deze situaties zijn de oppervlakken, via de ruwheden, met elkaar in contact waardoor ruwheidsinteracties en oppervlakteadhesie de wrijving bepalen. In dit proefschrift is een grenssmering-wrijvingsmodel beschreven op basis van deze twee mechanismen. Zowel numeriek als experimenteel is het wrijvingsmodel bestudeerd en gevalideerd.

Om de gevormde tribofilms tijdens grenssmering te karakteriseren alsmede de dikte en chemische samenstelling van deze tribofilms te kwantificeren is AFM, FIB-SAM en XPS ingezet. Daarnaast zijn nano indentaties uitgevoerd om de mechanische eigenschappen van de gevormde tribofilms te bepalen.

$\mathrm{Er}$ is een numeriek model ontwikkeld op basis van de semi-analytische methode (SAM) om de tangentiële krachten veroorzaakt door ruwheidsinteracties te kunnen berekenen. Elasto-plastische vervormingen van zowel de tribofilm als substraat zijn hierbij meegenomen. De effecten van tribofilmdikte, interferentie en mechanische eigenschappen van de tribofilm op wrijving zijn bestudeerd. De gevoeligheid van SAM-berekening van het werkelijk contactoppervlak is onderzocht door het werkelijk contactoppervlak van een twee dimensionaal sinus vormig oppervlak in contact met een vlak analytisch te berekenen als ook met de SAM. Op basis van de verkregen resultaten is een extrapolatie voorgesteld om een betere schatting te krijgen van het door SAM berekende werkelijke contactoppervlak.

Ten slotte is het effect van de adhesieve krachten en ruwheidsinteracties gecombineerd en de totale wrijving berekend. De resultaten werden gevalideerd door pinon- schijf testresultaten.

Het proefschrift bestaat uit twee delen. Het eerste deel (deel A) vat het werk samen in zes hoofdstukken. Het tweede deel (deel B) geeft een overzicht van de tijdschriftartikelen waarin de details staan beschreven. 


\section{CONTENTS}

\section{Part A}

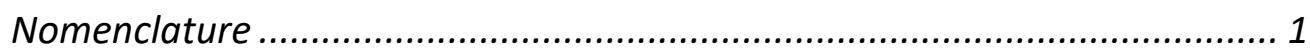

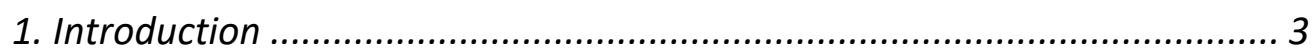

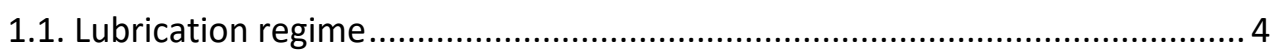

1.2. Friction mechanisms in boundary lubrication .............................................. 6

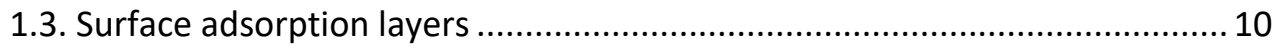

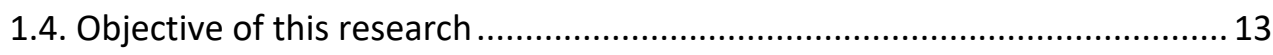

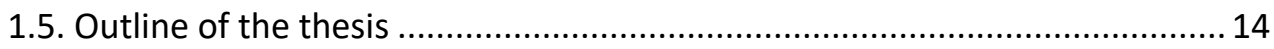

2. Morphology, chemical and mechanical properties of the triboflim ..........17

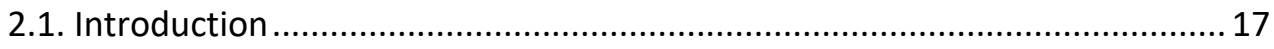

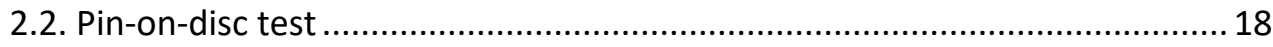

2.3. Morphology and thickness of the tribofilms.............................................. 21

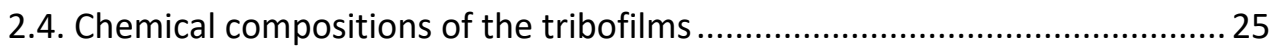

2.5. Elastic modulus and nanoscale hardness of the tribofilms............................ 27

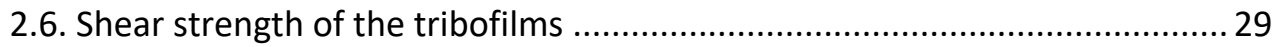

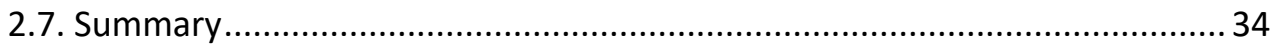

3. A single asperity sliding friction model .................................................. 39

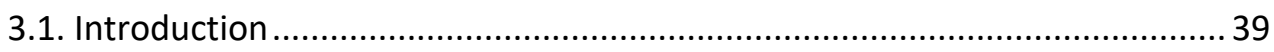

3.2. Development of single asperity sliding model.............................................. 40

3.3. FEM verification of displacement-driven contact model.................................4 47

3.4. Effects of interference depth and tribofilm properties on sliding friction .... 52

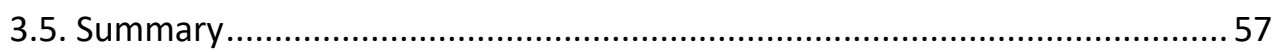

4. Real contact area in rough surface contact ............................................ 59

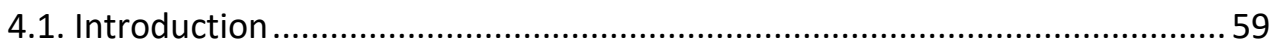


4.2. Real contact area between a wavy surface and a flat ..............................60

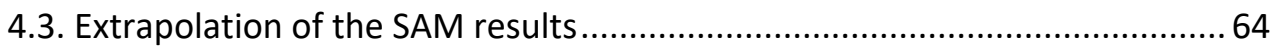

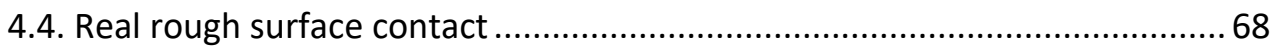

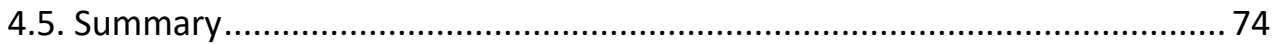

5. A combined friction model for boundary lubrication ......................... 77

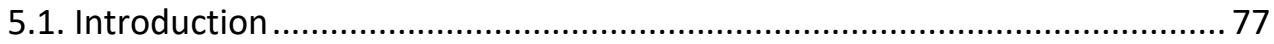

5.2. Calculation of the deformation force .............................................. 77

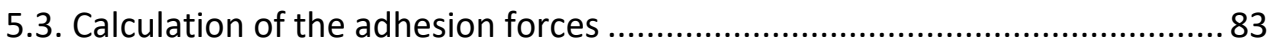

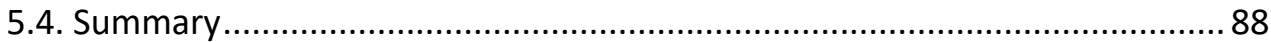

6. Conclusions and recommendations .......................................... 89

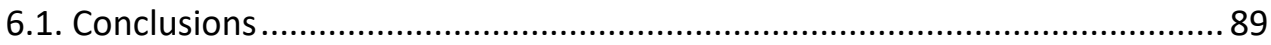

6.2. Recommendations for future research .............................................. 90

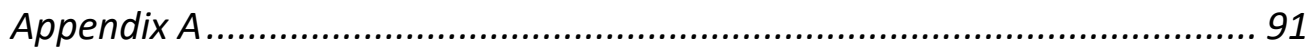




\section{Part B}

Paper A. Wang, C., \& Schipper, D. J. (2018). On an elastoplastic sliding model for a coated single asperity. Lubricants, 6(4), 96.

Paper B. Wang, C., \& Schipper, D. J. (2020). A numerical-analytical approach to determining the real contact area of rough surface contact. TribologyMaterials, Surfaces \& Interfaces, 1-11.

Paper C. Wang, C., Gojzewski, H., \& Schipper, D. J. (2020). A multi-technique characterization of the tribofilm formed by a fully formulated CVT fluid. Tribology International, 146, 106201.

Paper D. Wang, C., \& Schipper, D. J. (2021). A sliding dry friction model considering surface plastic deformation and adhesion. To be submitted. 

Part A 



\section{Nomenclature}

\begin{tabular}{|c|c|c|}
\hline$A_{c}$ & Contact area (hertzian) & $m^{2}$ \\
\hline$A_{p}$ & Projected area of the remaining deformation & $m^{2}$ \\
\hline$A_{r}$ & Real contact area & $\mathrm{m}^{2}$ \\
\hline $\boldsymbol{E}$ & Young's modulus of tribofilm & $\mathrm{Pa}$ \\
\hline $\boldsymbol{E}^{*}$ & Reduced modulus of elasticity & $\mathrm{Pa}$ \\
\hline $\boldsymbol{E}_{\boldsymbol{c}}$ & Young's modulus of cantilever & $\mathrm{Pa}$ \\
\hline $\boldsymbol{F}_{\boldsymbol{a}}$ & Friction force contributed by surface adhesion & $\mathrm{N}$ \\
\hline $\boldsymbol{F}_{a d v}$ & Adhesion force & $\mathrm{N}$ \\
\hline$F_{\text {contact }}$ & Total force during contact & $\mathrm{N}$ \\
\hline $\boldsymbol{F}_{d}$ & Friction force contributed by deformation & $\mathrm{N}$ \\
\hline$F_{D M T}$ & Applied load based on DMT model & $\mathrm{N}$ \\
\hline$F_{l}$ & Lateral load & $\mathrm{N}$ \\
\hline$F_{n}$ & Normal force & $\mathrm{N}$ \\
\hline$G_{c}$ & Shear modulus of cantilever & $\mathrm{Pa}$ \\
\hline $\boldsymbol{h}$ & Height of tip & $\mathrm{m}$ \\
\hline $\boldsymbol{h}_{0}$ & Initial gap between two surfaces & $\mathrm{m}$ \\
\hline$h_{\min }$ & Minimum film thickness & $\mathrm{m}$ \\
\hline $\boldsymbol{H}$ & Hardness of tribofilm & $\mathrm{Pa}$ \\
\hline $\boldsymbol{k}_{l}$ & Cantilever spring constant (lateral) & $\mathrm{N} / \mathrm{m}$ \\
\hline $\boldsymbol{k}_{n}$ & Cantilever spring constant (normal) & $\mathrm{N} / \mathrm{m}$ \\
\hline $\boldsymbol{l}$ & $\begin{array}{l}\text { Length of cantilever (from the end of the chip to the apex of the } \\
\text { tip) }\end{array}$ & $\mathrm{m}$ \\
\hline
\end{tabular}




\begin{tabular}{|c|c|c|}
\hline$p$ & Pressure distribution & $\mathrm{Pa}$ \\
\hline $\boldsymbol{R}$ & Radius of tip & $\mathrm{m}$ \\
\hline $\boldsymbol{R}_{\boldsymbol{q}}$ & Root mean squared profile roughness & $\mathrm{m}$ \\
\hline$S_{l}$ & Sensitivity of AFM optical system (lateral) & $\mathrm{V} / \mathrm{m}$ \\
\hline$S_{n}$ & Sensitivity of AFM optical system (normal) & $\mathrm{V} / \mathrm{m}$ \\
\hline $\boldsymbol{t}$ & Thickness of cantilever & $\mathrm{m}$ \\
\hline $\boldsymbol{u}_{\boldsymbol{e}}$ & Surface displacement caused by elastic deformation & $\mathrm{m}$ \\
\hline $\boldsymbol{u}_{r}$ & Surface displacement caused by residual deformation & $\mathrm{m}$ \\
\hline$V_{l}$ & Photo-potential of PSPD (lateral) & $\mathrm{V}$ \\
\hline$V_{n}$ & Photo-potential of PSPD (normal) & $\mathrm{V}$ \\
\hline $\boldsymbol{w}$ & Width of cantilever & $\mathrm{m}$ \\
\hline $\boldsymbol{\delta}$ & Indentation depth & $\mathrm{m}$ \\
\hline $\boldsymbol{\delta}_{\mathrm{z}}$ & Rigid body displacement in $z$ direction & $\mathrm{m}$ \\
\hline$\Delta$ & Amplitude of sinusoidal waviness & $\mathrm{m}$ \\
\hline$\lambda$ & Lambda ratio & 1 \\
\hline $\boldsymbol{v}$ & Poisson's ratio & 1 \\
\hline $\boldsymbol{\tau}$ & Shear strength of tribofilm & $\mathrm{Pa}$ \\
\hline
\end{tabular}




\section{Introduction}

In this chapter, the scope and the theory framework of the thesis are discussed. The lubrication regimes, the friction mechanisms of boundary lubrication and the surface adsorption layers are briefly reviewed. The research objectives are subsequently defined. Finally, the outline of this thesis is given, linking the first and second part of the thesis.

Friction is defined as the force resisting the relative motion of two surfaces in contact sliding against each other [1]. During this process, kinetic energy is converted into thermal energy, i.e. work is converted to heat.

Friction influences the society in both good and bad ways. As a dissipative process, friction is unwanted on many occasions due to energy loss and subsequent material damage. It is estimated that every year a quarter of energy is lost because of friction worldwide, which adds to $\mathrm{CO}_{2}$ emissions and global warming [2]. On the other hand, higher friction levels are desired in certain cases. Brake pads and clamps rely on friction to decelerate cars; airport conveyor belts need friction to move luggage; without friction between shoes and ground, people cannot even walk.

Due to the significant role friction plays in day-to-day life, it has been studied for thousands of years. In ancient Greek and Roman times, Aristotle, Vitruvius, and Pliny the Elder already started to investigate the cause and mitigation of friction [3][4][5]. Research into friction was further pushed forward during the $15^{\text {th }}$ to $18^{\text {th }}$ centuries by da Vinci [6], Amontons, Coulomb [7] and other tribologists in both theory and experiment. Three famous empirical laws were proposed [8]:

- Amontons' First Law: the force of friction is directly proportional to the applied load.

- Amontons' Second Law: the force of friction is independent of the apparent area of contact.

- Coulomb's Law of Friction: kinetic friction is independent of the sliding velocity.

While these three laws are well attested for many situations, the physical mechanisms behind them are not explicitly elaborated and therefore desired. Research to gain a deeper understanding of friction continues even today. Numerous 
manuscripts have been published, with many different friction mechanisms being proposed and studied. It is now clear that friction is a system response [9], while many factors including but not limited to material properties, surface roughness, temperature, load, and lubricating status, will affect the friction level of a given system.

In this thesis, the friction between two steel surfaces lubricated by a fully formulated oil is studied. The aim is to acquire a fundamental and physical understanding of the friction mechanisms of this tribosystem.

\subsection{Lubrication regime}

Stribeck [10] illustrated that the coefficient of friction is correlated to the viscosity of the lubricant and the velocity of the contacting surfaces, while being inversely correlated to the pressure exerted on the contact surfaces. With different combinations of these three terms, different lubrication regimes will occur.

A common way to determine the lubrication regime is through the lambda ratio [11], which is the ratio between minimum film thickness and the composite roughness of the surfaces. The lambda ratio is defined as:

$$
\lambda=\frac{h_{\text {min }}}{\sqrt{R_{q 1}^{2}+R_{q 2}^{2}}}
$$

Depending on the value of the lambda ratio, three regimes are defined from the Stribeck diagram: boundary lubrication, mixed lubrication and hydrodynamic lubrication, see Fig. 1.1. 


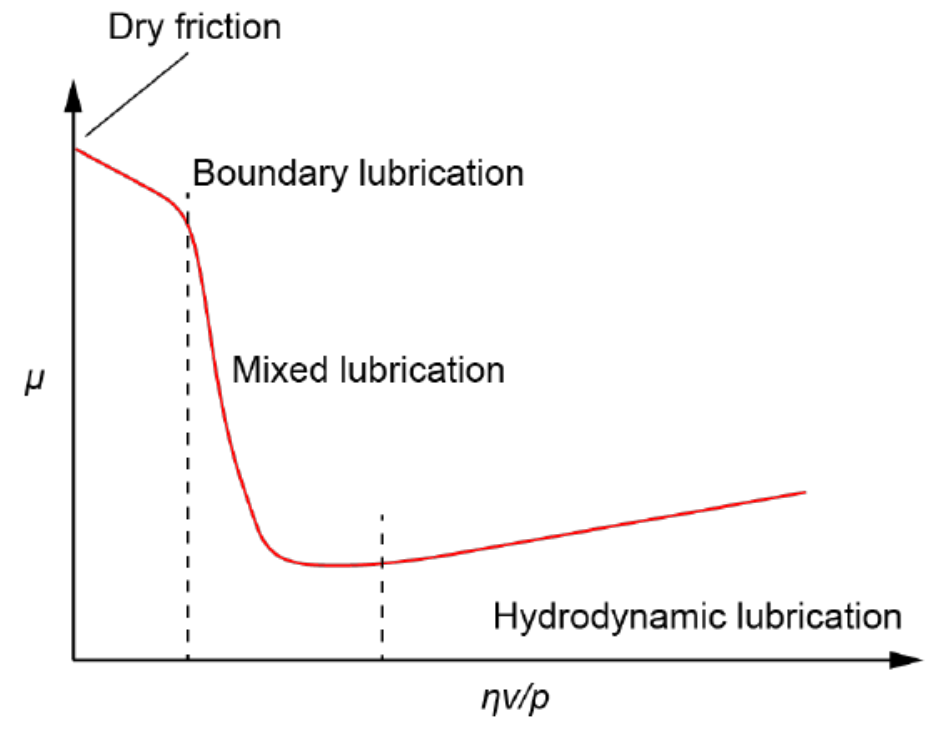

Fig. 1.1. Stribeck diagram and lubrication regimes [12].

(1) (Elasto-)hydrodynamic lubrication

If the lambda ratio is greater than 3 [13], which indicates a thick film compared to the roughness, the load will be borne entirely by the fluid film. In this case, friction force will be a function of the rheological properties of the lubricant under the operating conditions of load, temperature, shear rate, etc. The influence of the surface roughness will be negligible. Depending on the pressure generated in the lubricant and elastic properties of the contact surfaces, hydrodynamic or elastohydrodynamic lubrication may occur.

(2) Boundary lubrication

If the lambda ratio is lower than 1 [13], the film is too thin to separate the contact surfaces. Consequently, the load will be carried solely by the interacting asperities separated by boundary layers. The friction force originates mainly from this interacting process, while the shear properties of the boundary layers, whether formed by adsorption or reaction, become significant.

(3) Mixed lubrication

This is the intermediate regime between the (elasto-)hydrodynamic lubrication and boundary lubrication regimes, where the load is carried partly by the lubricant and 
partly by the interacting asperities. In this regime, the friction force will still be determined by the rheological properties of the lubricant but the local contact conditions of asperity interaction will need to be considered.

\subsection{Friction mechanisms in boundary lubrication}

In boundary lubrication, the influence of the hydrodynamic film on the friction force can be ignored. The solid to solid interactions between contacting surfaces dominate the friction mechanisms. The friction model proposed by Bowden and Tabor [14] states that the frictional force arises from two main sources: the force to supply the energy of deformation $\left(\boldsymbol{F}_{\boldsymbol{d}}\right)$ and the force needed to shear the adhered junctions $\left(\boldsymbol{F}_{\boldsymbol{a}}\right)$. Therefore, the total frictional force $(\boldsymbol{F})$ can be written as:

$$
F=F_{d}+F_{a}
$$

These two components of the total friction are introduced briefly below.

\subsubsection{Deformation force}

Plastic deformation consumes energy as friction when yielding occurs without energy feedback [9]. Two types of interactions may occur during the relative sliding of two contacting surfaces: the microscopic interaction where primarily caused by plastic deformation and displacement of the interacting surface asperities, and the more macroscopic interaction where the asperities of the harder surface plough grooves in the softer surface.

(1) Microscopic asperity interaction

Energy can be dissipated through the interactions of surface peaks, i.e. asperities, with no groove (macroscale deformation) being produced. When asperities slide over each other, local plastic deformation occurs and energy is dissipated as friction. This energy dissipation manner is studied extensively by researchers by means of single asperity modelling [15][16][17].

A brief illustration of this mechanism is shown in Fig. 1.2. Two single asperities slide over each other assuming no surface adhesion, see Fig. 1.2a. The tangential forces 
are shown in Fig. 1.2b for both pure elastic and elasto-plastic contact. In the pure elastic deformation case, the friction force curve acts in a centrosymmetric manner, and thus the net frictional force is zero. However, when plastic deformation is involved, asymmetry of the tangential force is observed and the net frictional force is non-zero. Evidently, the level of plastic deformation dominates the friction level of this mechanism.

\section{Sliding}
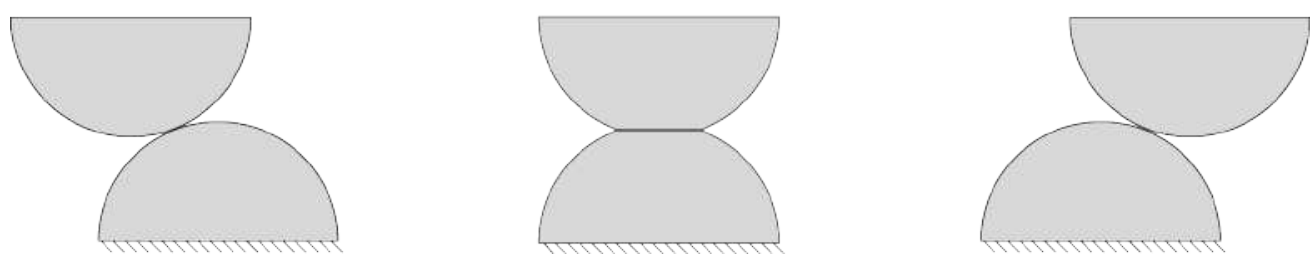

(a)

\section{Sliding}
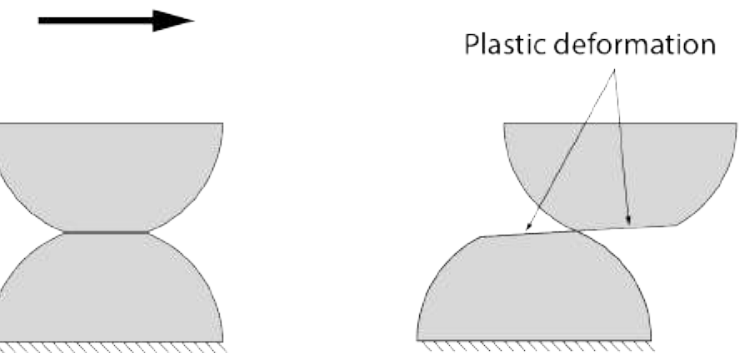

(b) 


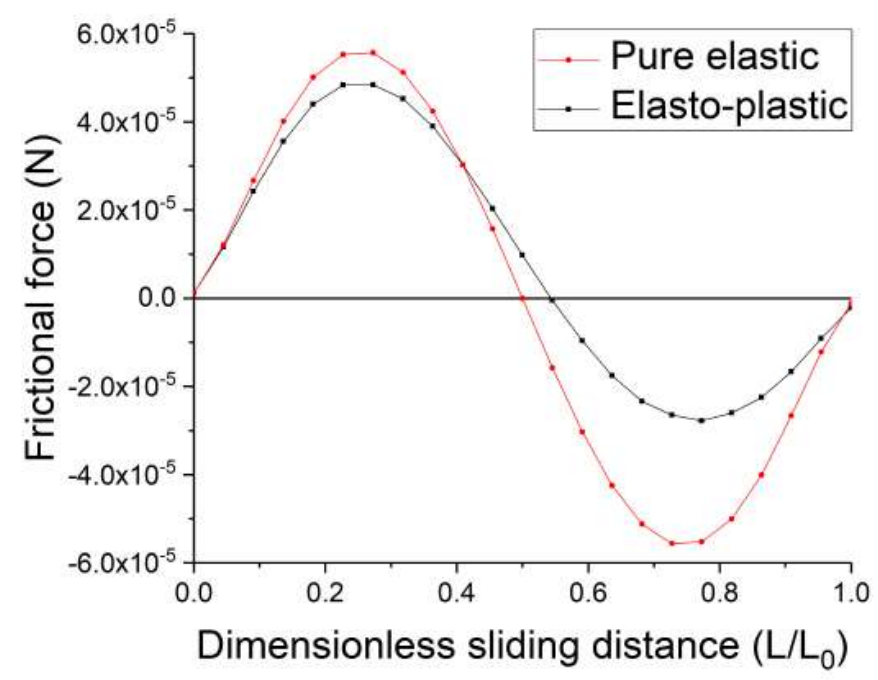

(c)

Fig. 1.2. Single asperity sliding: (a) elastic sliding; (b) elastoplastic sliding and (c) tangential forces during sliding.

(2) Macroscopic interaction

Macroscopic interaction of asperities occurs if one of the sliding surfaces is harder than the other, for instance the contact between sheet and die in sheet metal forming processes. The asperities of the harder surface may penetrate and plough into the softer surface and produce macroscopic grooves. Because of this ploughing deformation, a certain tangential force level is required to maintain motion, which is the frictional force.

A variety of models were developed with different asperity shapes. One of the most commonly used shapes is a conical asperity [8], which is shown in Fig. 1.3. In this case the hard cone asperity slides through the soft counter surface. Obviously, a tangential force, which is regarded as friction, is needed to maintain this movement. 


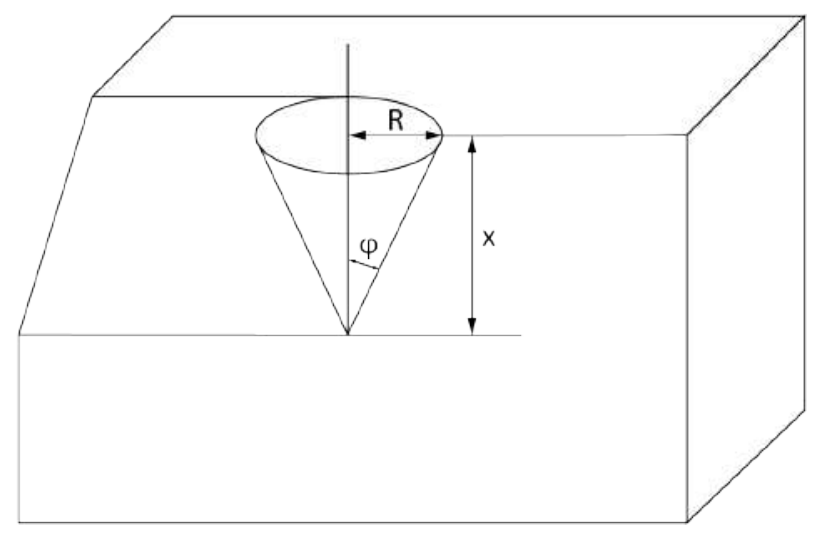

Fig. 1.3. Conical asperity ploughing model.

\subsubsection{Adhesive force}

When two surfaces are placed in contact via load, the contact will take place only at the tips of the asperities and will form local contact junctions. The sum of the area of all these junctions constitutes the real contact area $\left(\boldsymbol{A}_{\boldsymbol{r}}\right)$. For most materials under normal load, the real contact area is only a small fraction of the nominal contact area. The entire load will be supported by the real contact area instead of the nominal contact area. Consequently, local contact pressures are usually high.

Due to the high local contact pressure, adhesive bonds form on the real contact area caused by either physical or chemical interaction. Therefore, a tangential force is needed to shear the adhesive bonds to allow the two contacting surfaces to move relative to each other. This tangential force needed is the friction force caused by adhesion.

Because the adhesion force arises from the bonds at the surfaces, it shares the same nature of the inter-molecular forces and the interface may be as strong as the bulk materials that come into contact. Therefore, the friction force would depend on the bulk shear strength of the materials.

It is proposed by Bowden and Tabor [18] that 


$$
\boldsymbol{F}_{\boldsymbol{a}}=\boldsymbol{\tau} * \boldsymbol{A}_{\boldsymbol{r}}
$$

where $\boldsymbol{\tau}$ is the average shear strength of the contact interface and $\boldsymbol{A}_{\boldsymbol{r}}$ is the real contact area.

Note that the shear strength used in Eq. (1-3) is addressed to the contact interface. This shear strength may differ from the shear strength of the substrate material due to the formation of a surface adsorption layer. This topic will be deliberated in Section 1.3.

\subsubsection{The interaction between the two forces}

It should be noted that Eq. (1-2) is a rough approximation. It assumes implicitly that there is no interaction or coupling between $\boldsymbol{F}_{\boldsymbol{d}}$ and $\boldsymbol{F}_{\boldsymbol{a}}$. Further investigations [19] suggest that this assumption is too simplistic: these two terms are not strictly independent. Local deformations take place when both forces occur, which are difficult to distinguish. As a consequence, there may be a continuous interplay between the two components.

\subsection{Surface adsorption layers}

The lubrication mechanisms in boundary lubrication vary under different loads and temperature levels, and different surface layers are formed. With a relative low temperature (up to $150^{\circ} \mathrm{C}$ ) and high contact pressure ( $\sim \mathrm{GPa}$ ), the mechanism of "adsorption lubrication" is crucial [13]. The surface layers formed by this mechanism are called surface adsorption layers.

In this lubrication mechanism, mono-molecular layers form by adsorption of lubricant or lubricant additives on the worn surfaces. See Fig 1.4. This layer is thin enough that the mechanisms of asperity contact are identical to that of dry surfaces in contact. However, due to the different shear strength of the adsorption layer, the friction forces with and without the layer show different levels. 


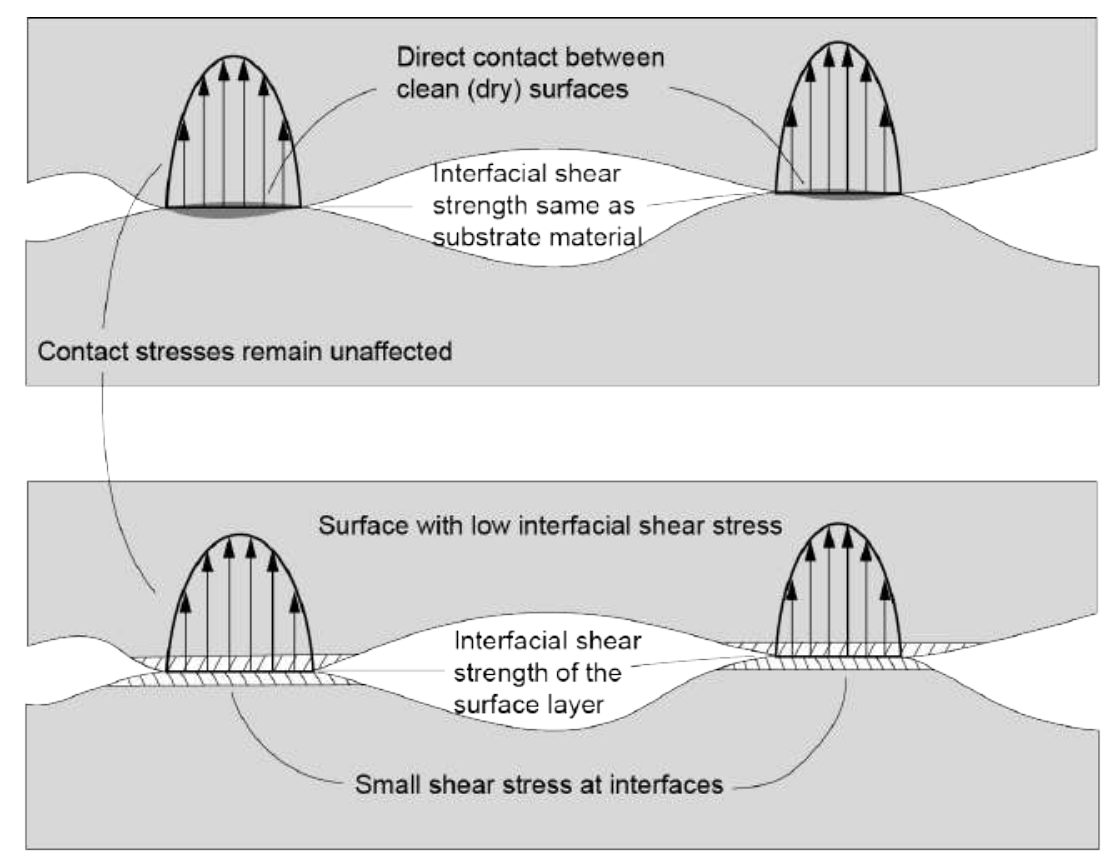

Fig. 1.4. Schematic of surface layers, reproduced from [13].

From the viewpoint of lubrication, adsorption layers can be divided into two basic categories: physically absorbed layers and chemically absorbed layers. Both will be introduced briefly below.

\subsubsection{Physically absorbed layer}

Physical adsorption is the classical form of adsorption that occurs at relatively low temperature. Molecules of the adsorbate attach or detach from a surface without any irreversible changes to the surface or the adsorbate, see Fig. 1.5. The bonding forces between surface and adsorbate are van der Waals and/or dispersion forces, as seen in Fig. 1.5. 


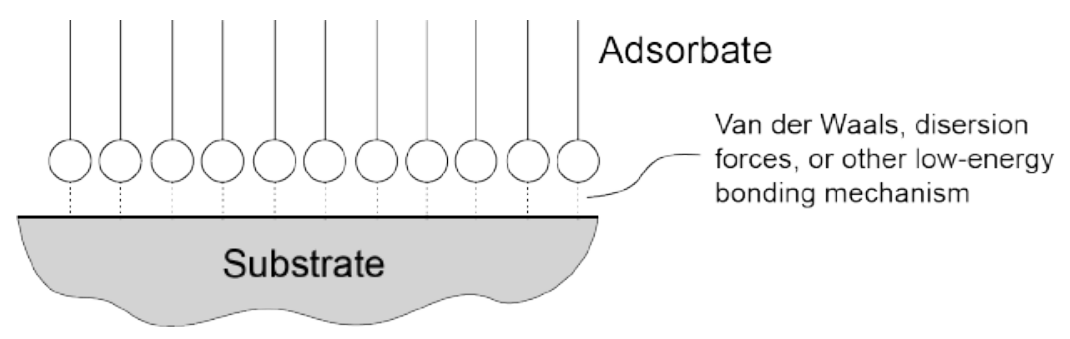

Fig. 1.5. Physically absorbed layer, reproduced from [13].

However, physical adsorption is effective only provided that temperatures do not exceed the ambient temperature too much: there is almost always an upper temperature limit to the physical adsorption process. An example of this temperature effect is shown in Fig. 1.6, showing the lubrication failure of two lubricants above critical temperatures [20].

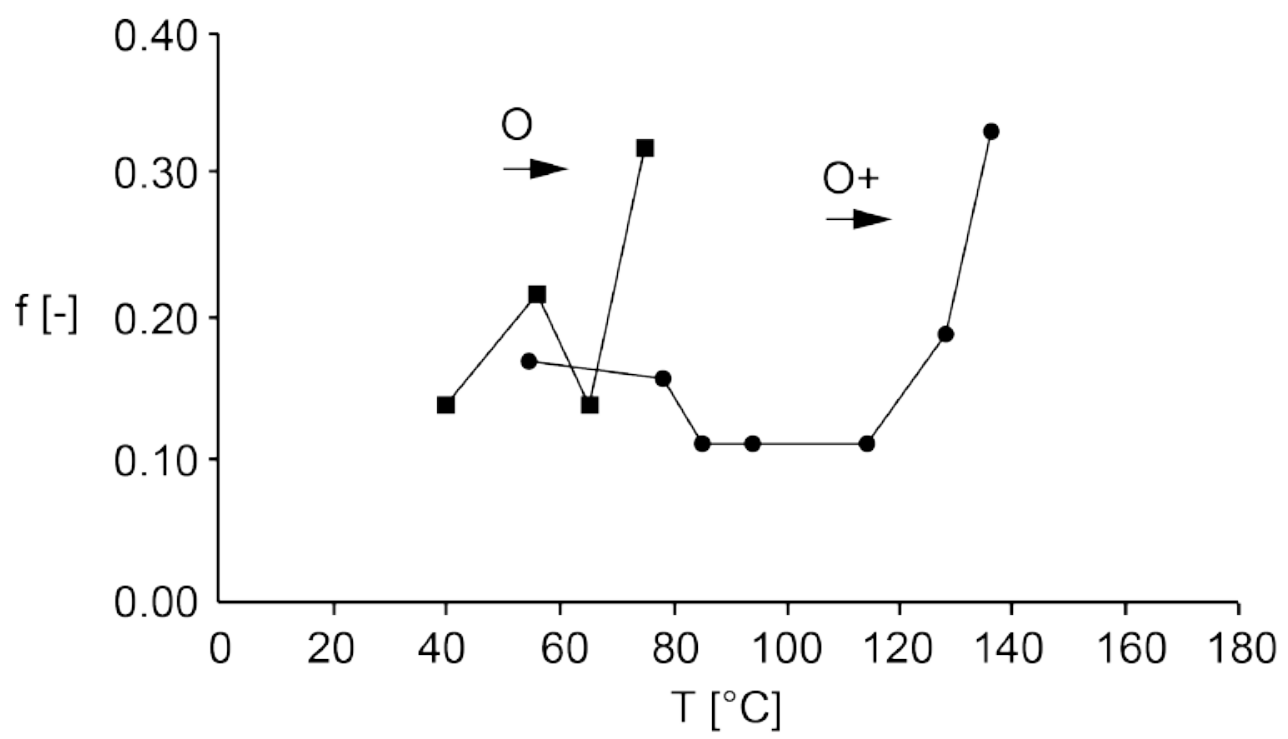

Fig. 1.6. Coefficient of friction as a function of the oil bath temperature measured with a pin-on-disc device, reproduced from [20]. 
Take O+ lubricant for example: it can be seen that the coefficient of friction constantly remains between 0.1 to 0.2 when the temperature is below $120^{\circ} \mathrm{C}$. However, for a higher temperature, a sharp transition occurs and the coefficient of friction increases to 0.35 . This indicates the failure of the physically absorbed layer, while direct metal to metal contact occurs.

\subsubsection{Chemically absorbed layer}

Chemical adsorption forms irreversible bonds between surfaces and adsorbates by chemical reactions, see Fig. 1.7. Most common engineering materials, e.g. iron, are reactive. Therefore, chemical adsorption is of great significance in adsorption lubrication. The strength of chemical bonding between the adsorbate and the substrate is usually firm. Even washing by strong solvents, which removes physical adsorption films, does not remove chemical adsorption films.

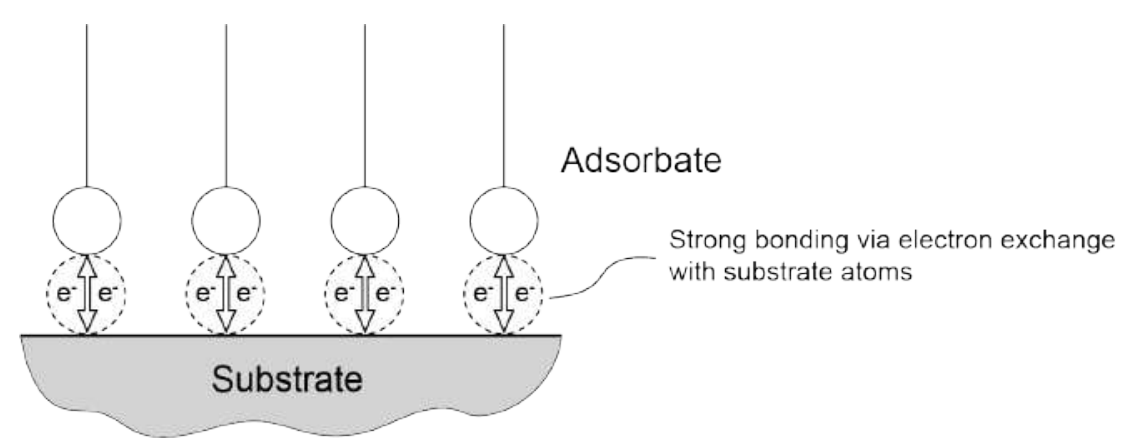

Fig. 1.7. Chemically absorbed layer, reproduced from [13].

Due to the stability of the chemical adsorption, lubricant additive packages are applied to form a chemical absorbed film with favourable properties for frictionmodifying and anti-wear purposes. Zinc dialkyldithiophosphates (ZDDP), for example, is a very widely used additive. The chemically absorbed layer formed by ZDDP during sliding process plays an important role in both friction and wear control [21].

\subsection{Objective of this research}


The main objective of this thesis is to gain a physical understanding of the friction in a boundary lubrication tribo-system lubricated by a CVT fluid, where a surface adsorption layer exists. To achieve this goal, experimental work and numerical modelling are combined. First, the surface adsorption layers are characterized in terms of morphology, chemical composition and mechanical properties. Subsequently, the contribution of deformation force and adhesive force respectively to the total friction is investigated. The modelling results are validated by pin-on-disc tests. The principal objectives are summarized as follows:

- Determine the real contact area of two rough surfaces with a predefined normal load.

- Investigate the morphological, chemical and mechanical properties of the surface adsorption layer generated by a CVT fluid to determine the adhesive force.

- Determine the deformation forces generated by asperity interactions by a single asperity sliding model, ignoring the surface adhesion force.

- Model the friction force and validate the results by experiments.

\subsection{Outline of the thesis}

This thesis is composed of two parts. The first part is a summary of the whole work and consists of six chapters, while the second part provides the published papers coming from the findings of this research.

In the first part, Chapter 1 explains boundary lubrication and its friction mechanisms, as well as the surface adsorption layer. Chapter $\mathbf{2}$ is a summary of the experimental work on the surface adsorption layer itself, where the morphological, chemical and mechanical properties of the surface layer are reported. Chapter $\mathbf{3}$ focuses on the calculation of the deformation force caused by asperity interactions using a single asperity sliding model. Chapter 4 introduces a method to determine the real contact area of rough surface contact, for the calculation of the adhesive force. In Chapter 5, findings in previous chapters are combined, and friction contributed by deformation forces and adhesion forces is calculated separately and compared with the experimental results. A summary of conclusions and recommendations for future research is presented in Chapter 6. 
The second part consists of four papers related to the research. The relation between the papers in the second part and the chapters in the first part of this thesis is illustrated in Fig. 1.8.

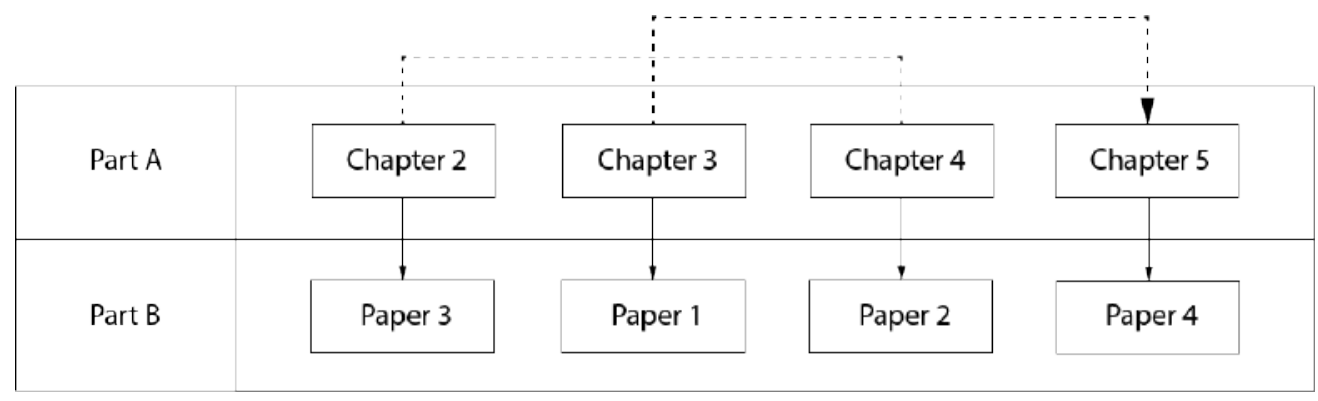

Fig. 1.8. Schematic outline of the thesis.

\section{References}

[1] Menezes, P. L., Nosonovsky, M., Ingole, S. P., Kailas, S. V., \& Lovell, M. R. (Eds.). (2013). Tribology for scientists and engineers (pp. 3-4). New York: Springer.

[2] Holmberg, K., \& Erdemir, A. (2017). Influence of tribology on global energy consumption, costs and emissions. Friction, 5(3), 263-284.

[3] Chatterjee, S. (2008). Tribological properties of pseudo-elastic nickel-titanium. University of California, Los Angeles.

[4] Tipler, P. A., \& Mosca, G. (2007). Physics for scientists and engineers. Macmillan.

[5] Sambursky, S. (2014). The physical world of late antiquity. Princeton University Press.

[6] Hutchings, I. M. (2016). Leonardo da Vinci's studies of friction. Wear, 360, 5166.

[7] Popova, E., \& Popov, V. L. (2015). The research works of Coulomb and Amontons and generalized laws of friction. Friction, 3(2), 183-190.

[8] Hutchings, I., \& Shipway, P. (2017). Tribology: friction and wear of engineering materials. Butterworth-Heinemann.

[9] Bhushan, B. (2013). Introduction to tribology. John Wiley \& Sons.

[10] Stribeck, R. (1902). Characteristics of plain and roller bearings. Zeit. VDI, 46. 
[11] Tallian, T. E. (1967). On competing failure modes in rolling contact. ASLE transactions, 10(4), 418-439.

[12] Dowson, D. (1978). History of tribology. Addison-Wesley Longman Limited.

[13] Stachowiak, G., \& Batchelor, A. W. (2013). Engineering tribology. ButterworthHeinemann.

[14] Bowden, F. P., Bowden, F. P., \& Tabor, D. (2001). The friction and lubrication of solids (Vol. 1). Oxford University Press.

[15] Boucly, V., Nélias D., \& Green, I. (2007). Modeling of the rolling and sliding contact between two asperities. Journal of Tribology, 129(2), 235-245.

[16] Mulvihill, D. M., Kartal, M. E., Nowell, D., \& Hills, D. A. (2011). An elastic-plastic asperity interaction model for sliding friction. Tribology International, 44(12), 1679-1694.

[17] Jackson, R. L., Duvvuru, R. S., Meghani, H., \& Mahajan, M. (2007). An analysis of elasto-plastic sliding spherical asperity interaction. Wear, 262(1-2), 210-219.

[18] Bowden, F. P., \& Tabor, D. (1973). Friction: an introduction to tribology. RE Krieger Publishing Company.

[19] Bowden, F. P., \& Young, J. E. (1951). Friction of clean metals and the influence of adsorbed films. Proceedings of the Royal Society of London. Series A. Mathematical and Physical Sciences, 208(1094), 311-325.

[20] van der Heide, E. (2002). Lubricant failure in sheet metal forming processes. University of Twente.

[21] Spikes, H. (2004). The history and mechanisms of ZDDP. Tribology Letters, 17(3), 469-489. 


\section{Morphology, chemical and mechanical properties of the triboflim}

In this chapter, the experimental works are summarized briefly. The friction levels of a boundary lubricated sliding pair were tested on a pin-on-disc tribo-tester. The tribofilms formed on the contact surfaces were analyzed by multiple techniques. The morphology and thickness of the tribofilms were measured by AFM and FIB-SEM. The chemical and mechanical properties were subsequently analyzed by XPS and AFM/LFM. The friction levels of the tribosystem were linked to the properties of the tribofilms.

\subsection{Introduction}

Tribofilms, as one type of chemical absorbed surface layers, are long been regarded as crucial in friction and wear control of boundary lubrication, where film thicknesses are not sufficient to separate surfaces, and asperity to asperity contacts occur. Generated by chemical reactions between additives and contact surfaces, tribofilms are constantly formed and removed in boundary lubrication and serve as selfsacrificing layers. In the past decades, numerousresearch papers have been published investigating the role that tribofilms play in the tribosystem and the mechanisms of their friction and wear control.

Morphology, thickness and chemical composition of the tribofilms are investigated by researchers, due to their close linkage to the frictional and wear characteristics of the tribosystem. Various surface analytical techniques have been applied in this field. Scanning electron microscopy (SEM) [1]-[4] and atomic force microscopy (AFM) [5][8] are extensively employed for the morphology of the tribofilms. A focused ion beam microscope (FIB) is frequently used to measure the thickness of the tribofilms [9]-[12], by providing straightforward cross-section profile images. As to the chemical composition of tribofilms, energy dispersive X-ray spectroscopy (EDX) is commonly employed to carry out the qualitative analysis in combination with SEM [13][14][15]. X-ray photoelectron spectroscopy (XPS) and X-ray absorption near edge structure (XANES) also serve as powerful tools to analyze the chemical state of elements in tribofilms [16][17][18]. 
Further, the mechanical properties of the tribofilms are also of great concern. In microscale and nanoscale tribology, it is proposed that the friction is controlled by the effective shear strength of the contacting surface layers and the real contact area. [19][20][21]. In boundary lubrication with tribofilms existing, this theory is also widely supported by literature. Mortier et al. [22] suggested that some tribofilms have low shear strength properties and allowed shearing within the film structure itself, leading to lower friction. The shear strength of the ZDDP tribofilm were linked to their friction modifying effects by different research groups [23]-[26]. Therefore, investigating the mechanical properties of tribofilms helps understanding the friction in layered boundary lubrication. However, due to the small thicknesses (usually 50$200 \mathrm{~nm}$ ) and the non-uniform distributions of the tribofilms, research in this area is rather limited. The nano-hardness of tribofilms, which serves as an indirect indication of the shear strength of tribofilms, has been investigated by researchers. Bec et al. [27] performed in-situ imaging of different tribofilms and discovered that the hardness of the tribofilms varied in depth. For ZDDP, the hardness started from about $1 \mathrm{GPa}$ on the surface and increased to $3 \mathrm{GPa}$ at $20 \mathrm{~nm}$ depth. Aktary et al. [28] reported that the hardness of ZDDP tribofilms formed on AISI 52100 steel ranged from 2.3 to $5.0 \mathrm{GPa}$. Pereira et al. [29] measured the hardness of ZDDP tribofilms at different temperatures and found a 2-4.5 GPa range. As to the researches on shear strength of the tribofilms, Pidduck and Smith [30] proposed the idea of employing AFM and lateral force microscopy (LFM) to study the lateral force of the tribofilm. Kano and Yasuda [31] employed the AFM to study the shearing forces of the ZDDP tribofilm. However, only the electrical signals were presented, without being translated into forces.

In this chapter, a pin-on-disc tribosystem running under boundary lubrication is studied. The friction levels of the contact samples were measured under different temperatures, after which the post-test surfaces were investigated by a series of characterizing techniques. The morphology and thickness of the tribofilms and their chemical and mechanical properties were determined and linked to the friction levels.

\subsection{Pin-on-disc test}

A sliding boundary lubricated tribosystem was evaluated by a pin-on-disc tribometer. The sample used is a ball and disc pair, see Fig. 2.1. The steel ball is of $10 \mathrm{~mm}$ in 
diameter and made of AISI 52100 with hardness of 62-66 HRC. The centre-line average roughness $R_{a}$ is $20 \mathrm{~nm}$. The disc is of the same material as the ball (AISI 52100 ), with a centre-line average roughness $R_{a}$ of $120 \mathrm{~nm}$. Both the ball and the disc were ultrasonically cleaned by 2-propanol for $10 \mathrm{~min}$ prior to the test.

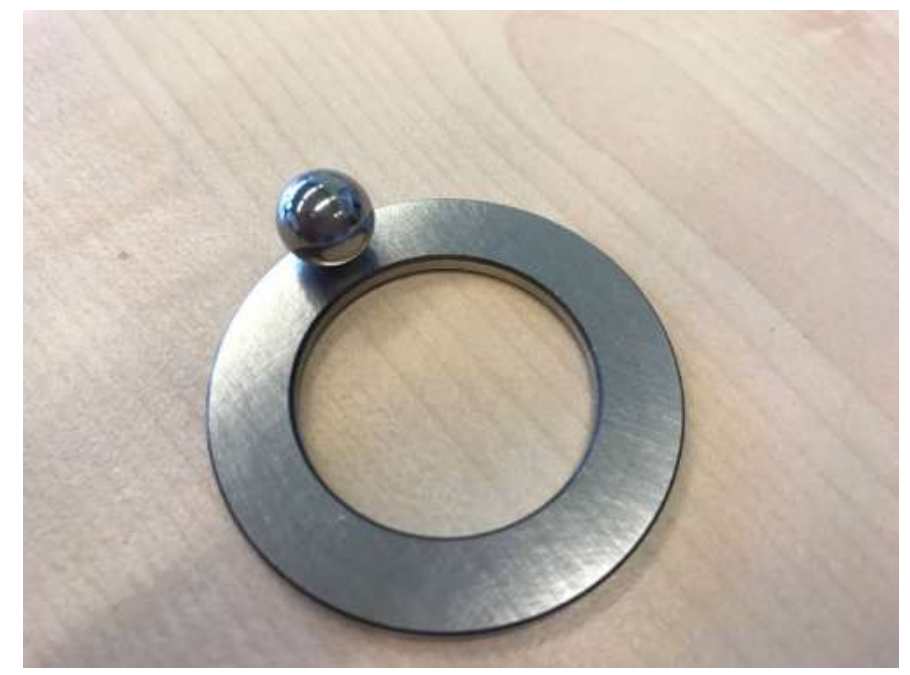

Fig. 2.1. Ball and disc samples used in the pin-on-disc test.

After the ball and disc were fitted to the machine, $10 \mathrm{~mL}$ lubricant (Shell NS-3) was applied and the temperature was raised to the set value without applying any load. When the system reached the test temperature, the normal load was applied and the test started. The tribological data, including lateral force and coefficient of friction, were monitored and recorded by the sensors. The detailed tests conditions are listed in Tab. 2.1. 
Tab. 2.1. Running conditions of the pin-on-disc test.

\begin{tabular}{cc}
\hline Parameter & Value \\
\hline Load $(\mathrm{N})$ & 10 \\
Max. Hertzian pressure $(\mathrm{GPa})$ & 1.01 \\
Avg. Hertzian pressure $(\mathrm{GPa})$ & 0.67 \\
Hertzian contact diameter $(\mu \mathrm{m})$ & 138 \\
Sliding velocity $(\mathrm{m} / \mathrm{s})$ & 0.1 \\
Oil temperature $\left({ }^{\circ} \mathrm{C}\right)$ & 80 to 150 \\
Duration $(\mathrm{h})$ & 12 \\
\hline
\end{tabular}

The steady state coefficients of friction of the tribosystem under different temperatures are shown in Fig. 2.2. It is evident that in the temperature range between 80 and $150{ }^{\circ} \mathrm{C}$ the coefficient of friction increases with increasing temperature. At $80^{\circ} \mathrm{C}$, a COF of 0.12 is observed. The COF rises smoothly with temperature. At $150^{\circ} \mathrm{C}$, the $\mathrm{COF}$ is 0.14 , more than $15 \%$ higher than at $80^{\circ} \mathrm{C}$.

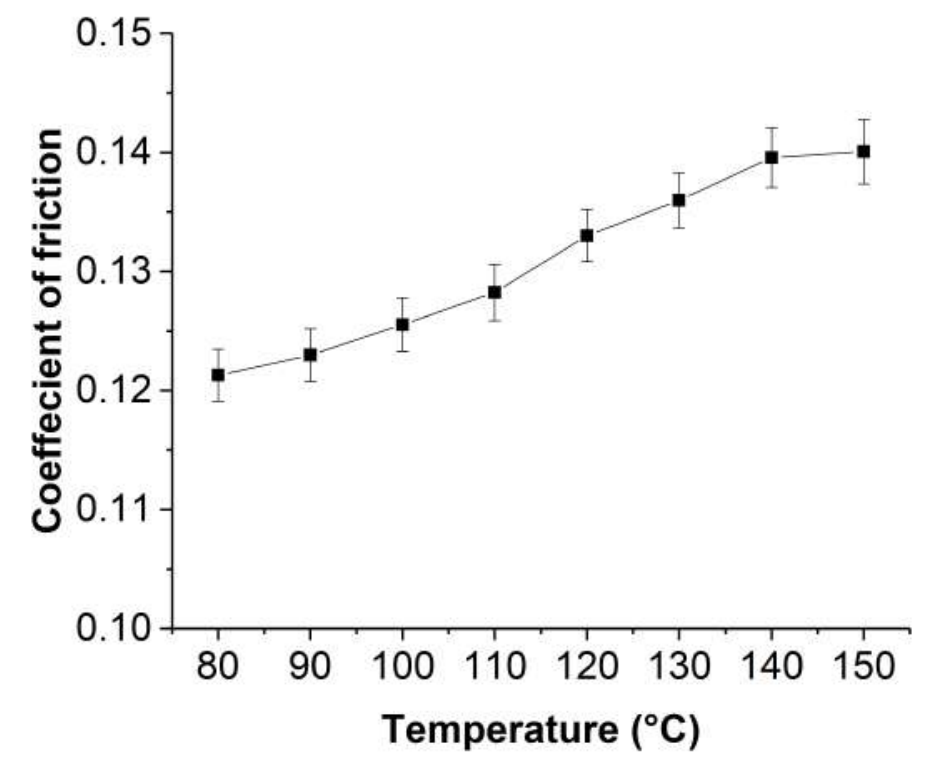

Fig. 2.2. The coefficient of friction at different temperatures. 
The difference in friction at different temperatures suggests that temperature plays a role in this tribological process. The reason may be attributed to the fact that the tribofilms formed at different temperatures differ in morphology, thickness, chemical contents or mechanical properties. To better understand this tribological process, further investigation on the tribofilms was carried out and is presented in the following sections.

\subsection{Morphology and thickness of the tribofilms}

To determine the morphology and thickness of the tribofilm, the wear scar on the post-test surface of the ball sample was analysed by AFM and SEM/FIB.

Representative z-height AFM images of post-test ball samples are shown in Fig. 2.3. The rubbing direction is along the $y$ axis. The corresponding cross-section profiles taken perpendicular to the sliding direction - are also presented for comparison. 

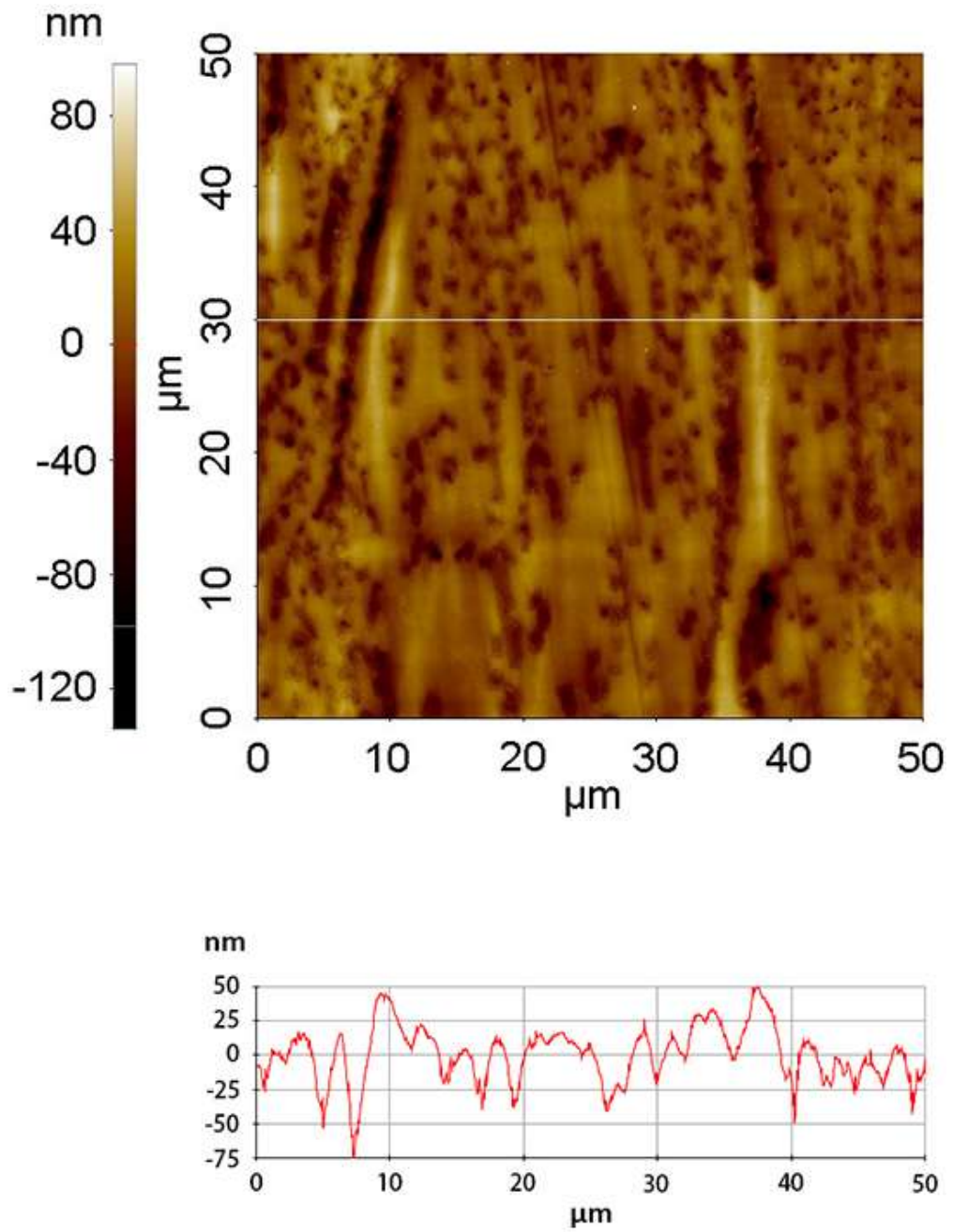

(a) 

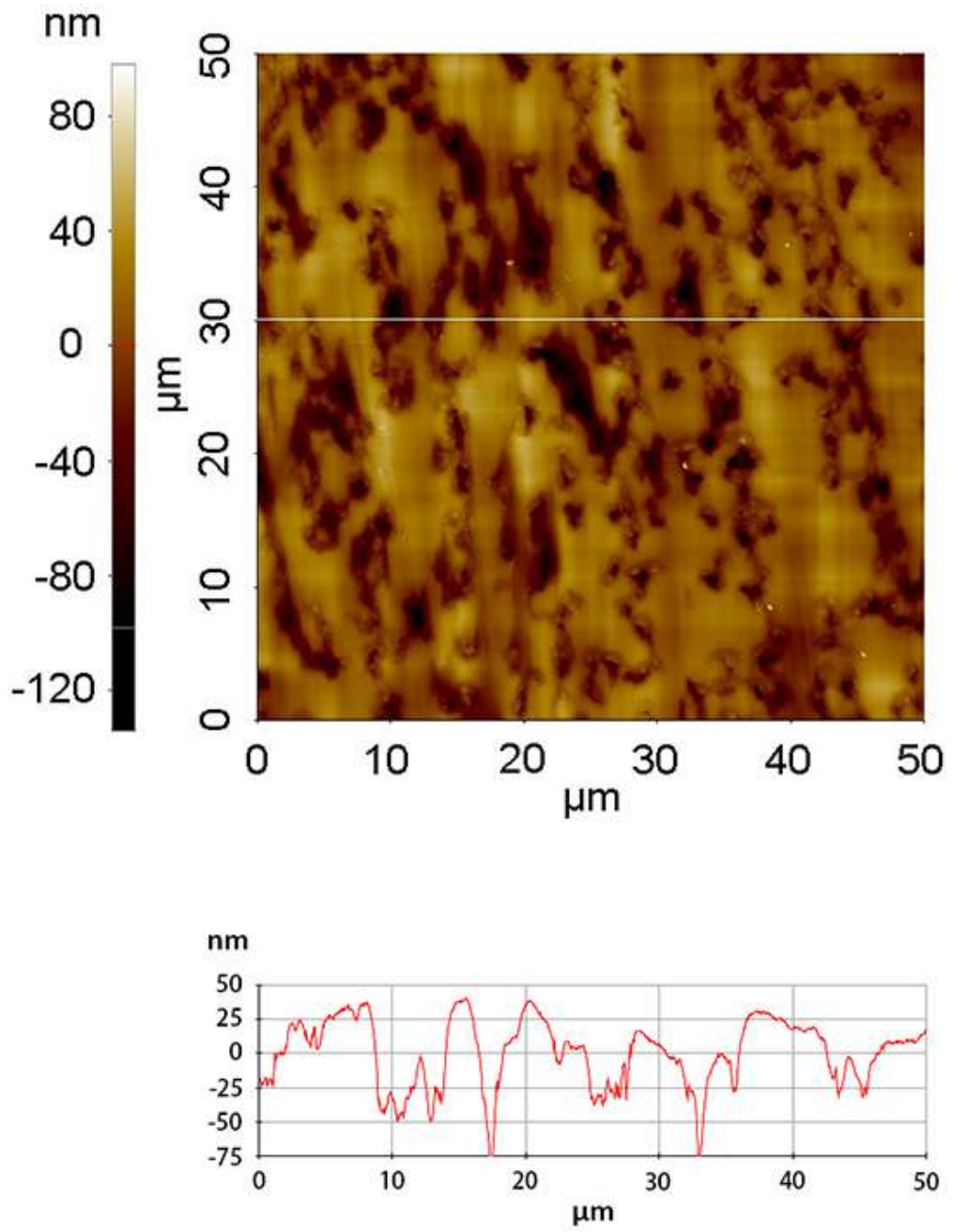

(b)

Fig. 2.3. AFM height images and topography profiles (along the white section lines) of the tribofilms formed at: (a) $80^{\circ} \mathrm{C}$ and (b) $150{ }^{\circ} \mathrm{C}$.

Similar morphology of the tribofilms formed at different temperatures are observed. The post-test surfaces are inhomogeneous and pad-like structures are observed along the sliding direction. The bright, elevated pads are tribofilms and the dark valleys is the steel substrate not being covered (this is further confirmed by the SEMFIB images below). Large tribofilm pads $(10-20 \mu \mathrm{m})$ form and elongate in the sliding direction, while small tribofilm pads $(1-5 \mu \mathrm{m})$ are also observed between the 
substrate valleys. For both samples, the height differences between peaks and valleys are around 100 to $125 \mathrm{~nm}$, which provide hints of the tribofilm thicknesses. It can be summarized that, because of minor differences of the tribofilm morphology formed at 80 and $150^{\circ} \mathrm{C}$, larger and more continuous tribofilm pads are formed on the wear scar at $150^{\circ} \mathrm{C}$ than at $80^{\circ} \mathrm{C}$. This can be identified by both the z-height images and the cross-section profiles.

A focused ion beam microscope was used to generate images of the cross-section of the tribofilm. Representative SEM images are presented in Fig. 2.4. It can be identified that the morphology and thickness of the tribofilms formed at different temperatures are rather similar. Most of the surfaces are covered with a darkcolored tribofilm, a small proportion of the surfaces are steel substrate without tribofilm. This is in good agreement with the AFM topography results. The thicknesses of the tribofilms are measured and labelled in Fig. 2.4. For both samples, the thicknesses of the tribofilms are around 120 to $135 \mathrm{~nm}$.

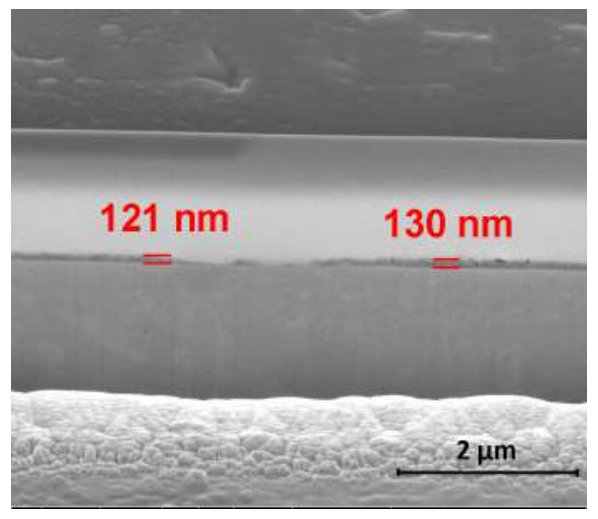

(a)

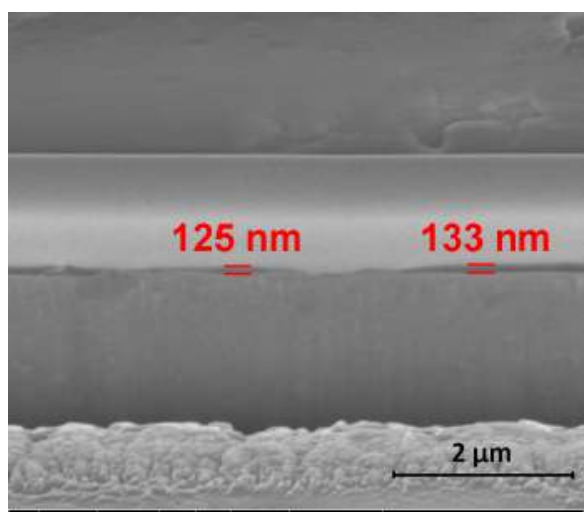

(b)

Fig. 2.4. FIB-SEM images of the tribofilms: (a) $80^{\circ} \mathrm{C}$ and (b) $150^{\circ} \mathrm{C}$.

To sum up, the morphology and thickness of the tribofilms formed at different temperatures show no significant differences. Therefore, the chemical and mechanical properties of the tribofilms may dominate the friction and need to be analysed. 


\subsection{Chemical compositions of the tribofilms}

The atom concentrations of the tribofilms were investigated by XPS. The depth profiles are shown in Fig. 2.5. It can be seen that the atom concentrations follow a similar trend: $\mathrm{P}$ and $\mathrm{Ca}$ decreases with depth and the Fe concentration increases with depth. At the depth of $125 \mathrm{~nm}, \mathrm{P}$ and Ca almost disappear in both cases, while the Fe concentrations go beyond $80 \%$. This suggests the tribofilm thicknesses for both $80^{\circ} \mathrm{C}$ and $150{ }^{\circ} \mathrm{C}$ are around $130 \mathrm{~nm}$, in good agreement with the AFM and FIB-SEM results. However, the element concentrations vary for the two samples tested at different temperatures. It can be seen that, at the surface, the tribofilm generated at $80{ }^{\circ} \mathrm{C}$ contains around $12.5 \%$ of $\mathrm{Fe}$, and almost $40 \%$ in the tribofilm generated at $150{ }^{\circ} \mathrm{C}$. The concentrations of $\mathrm{P}$ and $\mathrm{Ca}$ are lower in the $150{ }^{\circ} \mathrm{C}$ case (both around $5 \%$ ), in contrast to the $80^{\circ} \mathrm{C}$ case (over $10 \%$ ). This clearly suggests that even though the chemical compositions of the tribofilms generated at $80^{\circ} \mathrm{C}$ and $150{ }^{\circ} \mathrm{C}$ are comparable, the concentrations of the elements are rather different. The tribofilm generated at $150{ }^{\circ} \mathrm{C}$ is more Fe rich than that generated at $80^{\circ} \mathrm{C}$. This will probably lead to different mechanical properties of the tribofilm and further affect the friction levels.

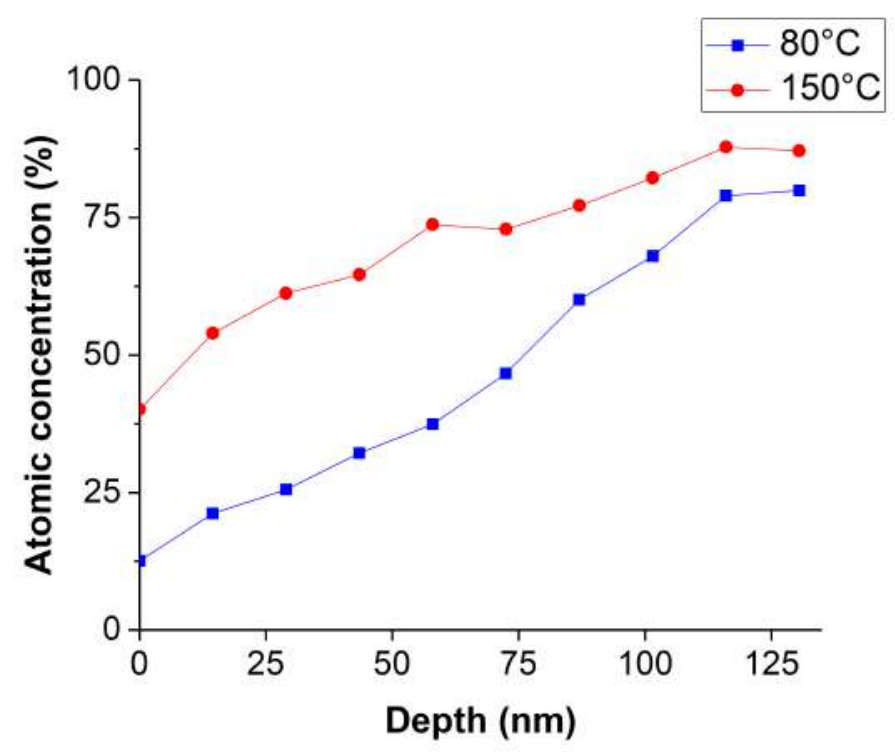

(a) 


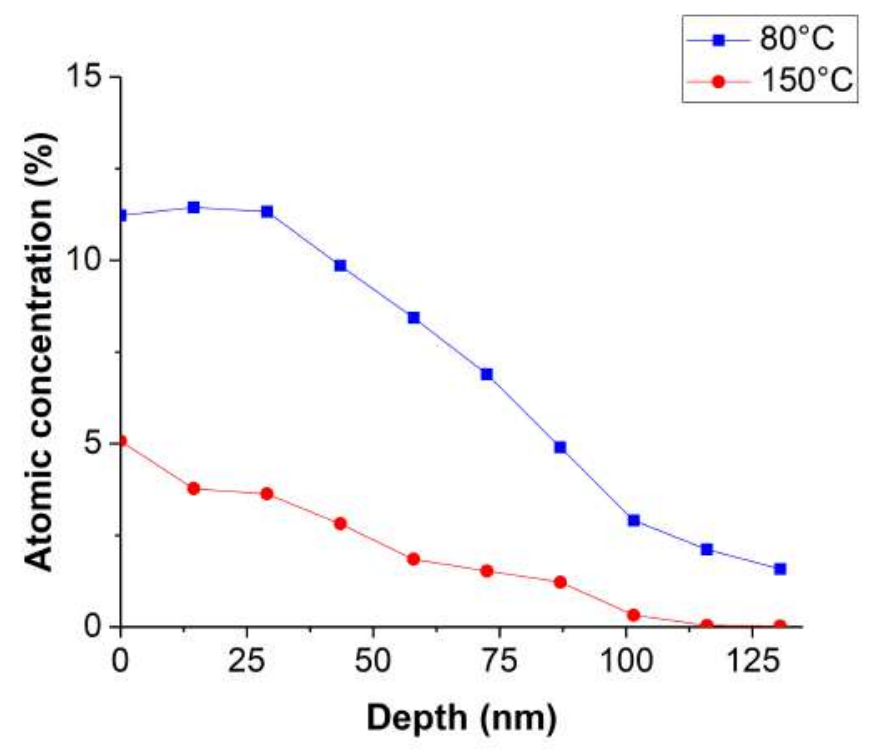

(b)

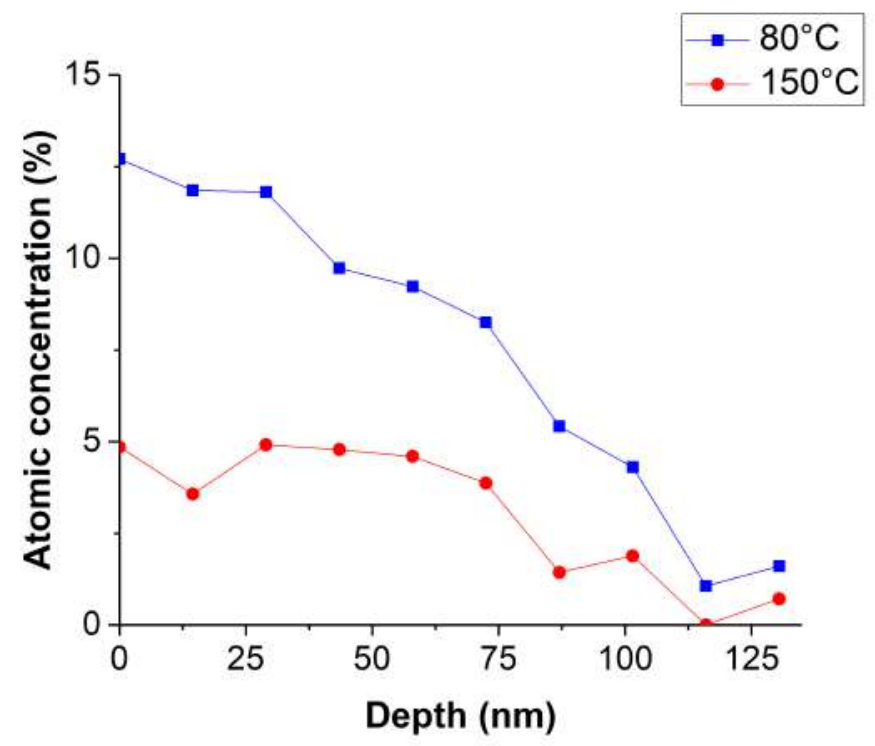

(c)

Fig. 2.5. XPS depth profile of atomic concentrations: (a) Fe, (b) Ca and (c) P. 
Therefore, the mechanical properties of the tribofilms need to be further characterized.

\subsection{Elastic modulus and nanoscale hardness of the tribofilms}

The elastic modulus and nanoscale hardness measurements of the tribofilm were conducted by AFM indentation.

The hardness, $\boldsymbol{H}$, was calculated using the following formula [32][33]:

$$
H=\frac{F_{n}}{A_{p}}=\frac{k_{n} S_{n} V_{n}}{A_{p}}
$$

where $\boldsymbol{F}_{\boldsymbol{n}}$ is the normal load, $\boldsymbol{A}_{\boldsymbol{p}}$ is the projected area of the remaining indentation dent, $\boldsymbol{V}_{\boldsymbol{n}}$ is the photo-potential measured vertically with a position sensitive photo detector (PSPD), and $\boldsymbol{S}_{\boldsymbol{n}}$ is the vertical sensitivity of the AFM optical system.

Fig. $\mathbf{2 . 6}$ represents an example of submicron scale visualization (AFM, contact mode) of tribofilm surfaces with residual plastic impression after AFM-tip indentation (dark area in the images). Due to the diamond AFM-tip coating, the geometry of the indenter varies from one cantilever to another, resulting in differences in the shape of the indentation profile (projected area). The tribofilm formed at $150{ }^{\circ} \mathrm{C}$ turned out to be of a high hardness; high normal loads needed to be applied to obtain a decent indentation hole with the AFM setup used. The calculated average nanoindentation hardness $\boldsymbol{H}$ is $4.7 \pm 1.1 \mathrm{GPa}$ for this sample. In contrast, for the tribofilm formed at at $80{ }^{\circ} \mathrm{C}$ the calculated average $\boldsymbol{H}$ is $2.7 \pm 0.7 \mathrm{GPa}$; this sample can evidently be deformed under lower normal loads. These values fall into ranges similar to the hardness of ZDDP tribofilms in [28]. 


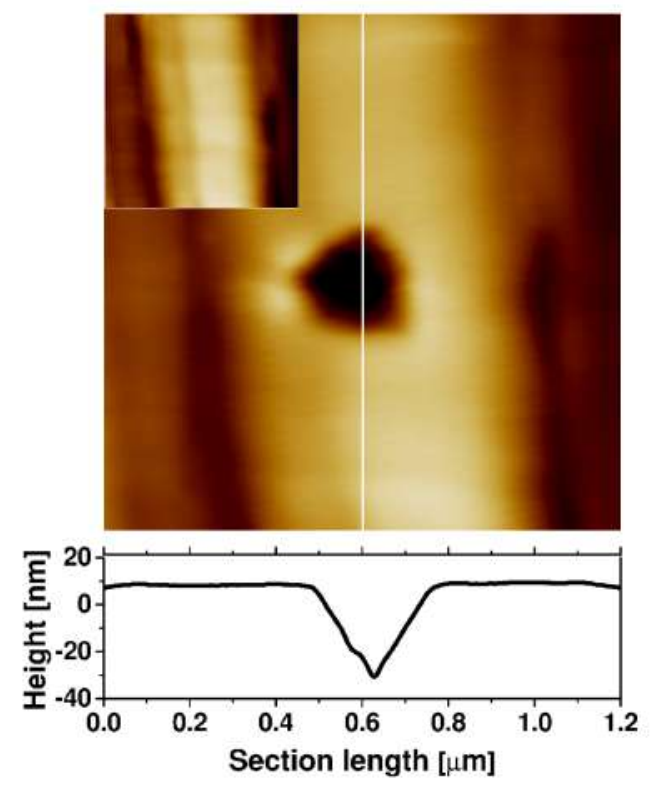

(a)

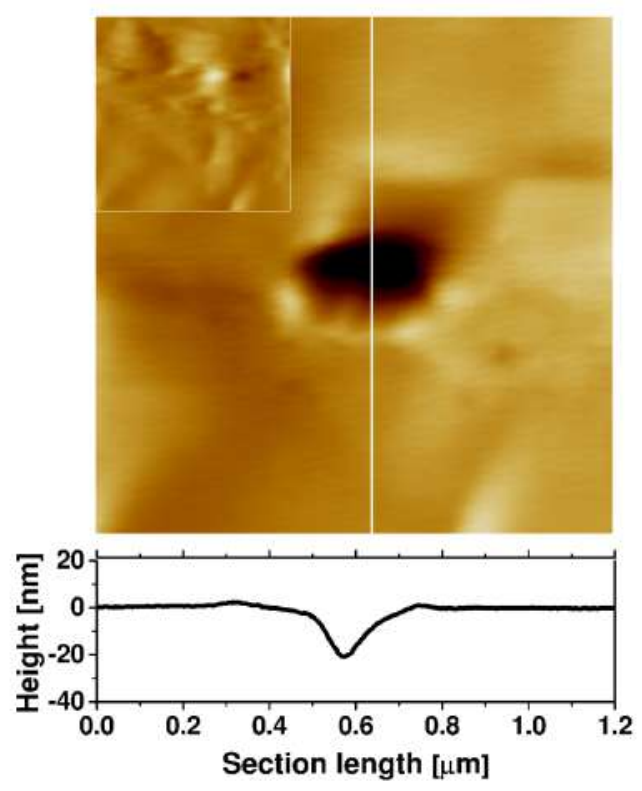

(b)

Fig. 2.6. AFM height images (contact mode) of the post-indented tribofilm surfaces tested at normal loads of (a) $52 \mu \mathrm{N}$ (film formed at $80^{\circ} \mathrm{C}$ ) and (b) $74 \mu \mathrm{N}$

(film formed at $150^{\circ} \mathrm{C}$ ). The insets represent the investigated area before indentation. Cross-section are plotted along the white section lines.

The Young's moduli values were calculated by the Derjaguin, Muller, and Toporov (DMT) model to quantify the tribofilm elasticity. In this model the deformed contact profile is the same as in the Hertz model, however with a higher applied load, $\boldsymbol{F}_{\boldsymbol{D M T}}$, due to an additional adhesion interaction. The following formula was used [34][35]:

$$
F_{D M T}=\frac{4}{3} \frac{E}{(1-v)} \sqrt{R} \delta^{\frac{3}{2}}+F_{a d h}
$$

The values of Young's modulus follow the trend of the hardness result. For the tribofilm formed at $150{ }^{\circ} \mathrm{C}$ the average $\boldsymbol{E}$ is $75 \pm 16 \mathrm{GPa}$, while for the tribofilm formed at $80{ }^{\circ} \mathrm{C}$ it is $56 \pm 14 \mathrm{GPa}$. These values are similar to the Young's modulus of 
ZDDP tribofilms reported in literature [42][43]. The $\boldsymbol{E}$ values distributions are shown in Fig. 2.7.

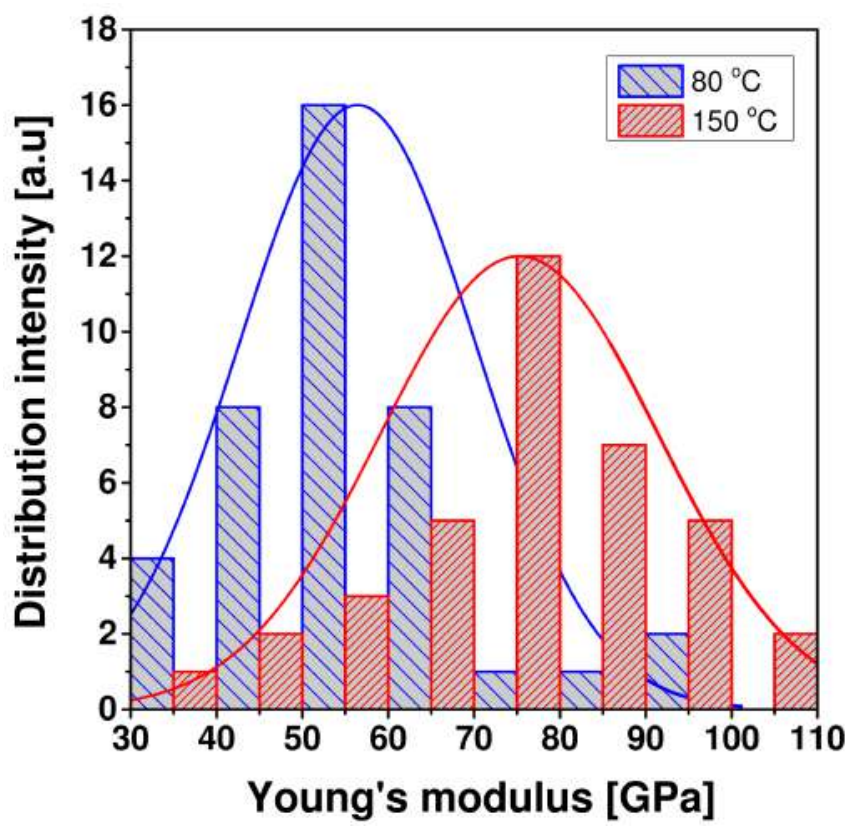

Fig. 2.7. Young's modulus values histogram for tribofilms formed at 80 and $150{ }^{\circ} \mathrm{C}$. The curves (fit of the normal curve to the distribution) are to guide the eye.

\subsection{Shear strength of the tribofilms}

The shear strengths of the tribofilm were measured by the AFM running under LFM mode. In this mode, the cantilever moves laterally parallel to the surface and is deflected sideways due to tip-sample interaction (friction). The lateral force can be calculated by the method proposed by Pietrement and Troyon [37]:

$$
F_{l}=\frac{E_{c} w t^{3}}{6 l^{2}(h+t / 2)} S_{n} V_{l}
$$


The contact area, $\boldsymbol{A}_{\boldsymbol{c}}$, and the mean contact pressure of the cantilever tip and the surface, $\overline{\boldsymbol{p}}$, can be calculated by the Hertzian contact model [39]:

$$
\begin{array}{ll}
A_{c}=\pi a^{2} & (2-4) \\
\bar{p} & =\frac{F_{n}}{\pi a^{2}}
\end{array}
$$

where $\boldsymbol{a}$ is the contact radius, which can be expressed as:

$$
a=\sqrt[3]{\frac{3 F_{n} R\left(1-v^{2}\right)}{4 E}}
$$

The shear strength then can be calculated as:

$$
\tau=\frac{F_{l}}{A_{c}}=\frac{1}{\pi a^{2}} * \frac{E_{c} w t^{3}}{6 l^{2}(h+t / 2)} S_{n} V_{l}
$$

In the lateral force measurement, three different normal loads $\boldsymbol{F}_{\boldsymbol{n}}$, were applied: 1 $\mu \mathrm{N}, 2 \mu \mathrm{N}$ and $5 \mu \mathrm{N}$. These loads were chosen in order that the top surface of the tribofilm was effectively "sheared" during the measurement instead of just elastically deformed. In addition, the shear forces were measured at three different sliding velocities, which are $0.01 \mathrm{~mm} / \mathrm{s}, 0.05 \mathrm{~mm} / \mathrm{s}$ and $0.25 \mathrm{~mm} / \mathrm{s}$.

The raw data of the lateral force measurements are electrical signals measured in volts. They are equal to $\boldsymbol{V}_{\boldsymbol{l}}$, representing the lateral torsion. An example image is presented in Fig. 2.8. The z-height image of the sample acquired at the same time is also presented for comparison. 


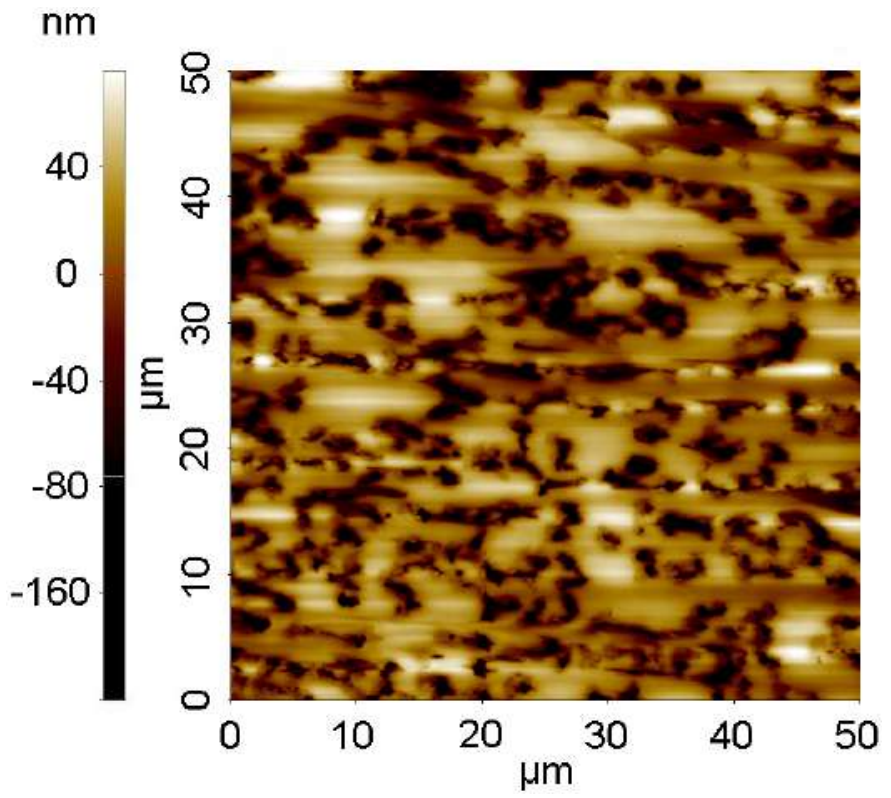

(a)

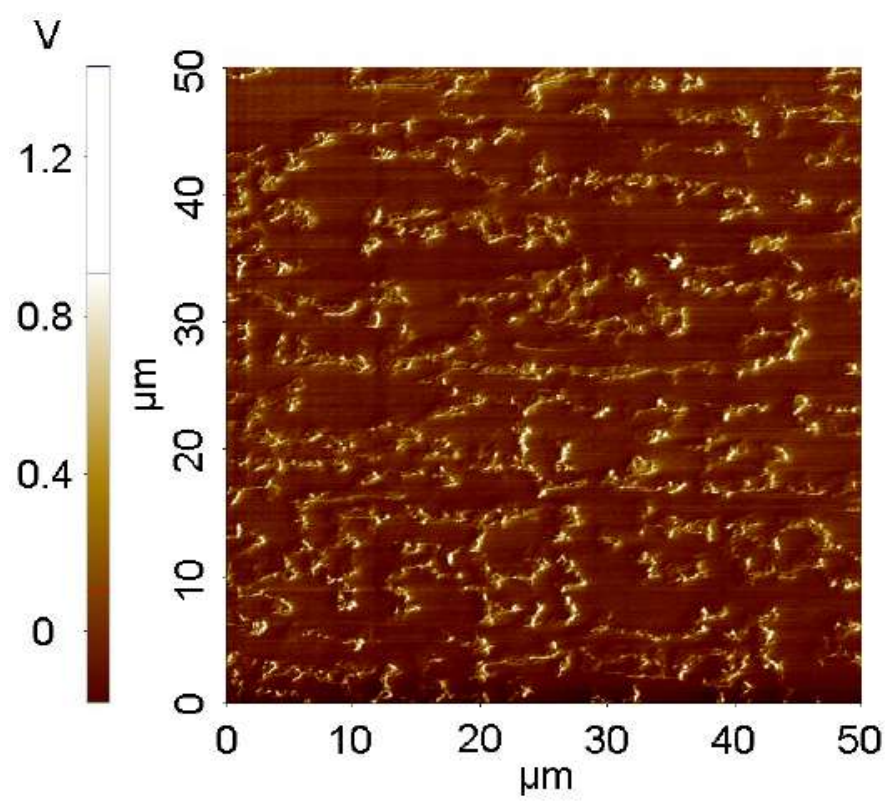

(b)

Fig. 2.8. AFM results of lateral force measurement. The normal load is $5 \mu \mathrm{N}$ and the shearing velocity is $0.25 \mathrm{~mm} / \mathrm{s}$ : (a) height image (tapping mode) and (b) representative PSPD lateral force (LFM) image. 
It can be noticed that the lateral force results show an identical pattern to the zheight result, implying that the lateral forces are influenced by the surface roughness. This is anticipated because when the tip travels through bumps or grooves, the tilting angle changes due to the surface tilting. However, in the relatively flat areas, the signals are rather steady. This indicates that the lateral force on these areas is almost constant, and can therefore be interpreted as the force for shearing the surface. A line profile of trace and retrace lateral forces can be seen in Fig. 2.9.

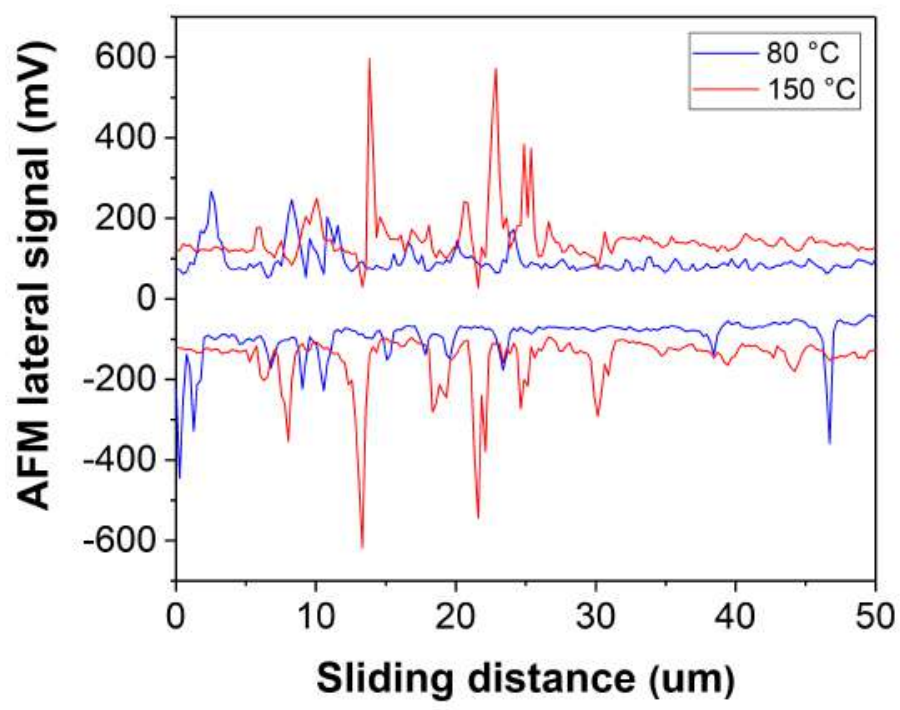

Fig. 2.9. Line profiles of the lateral force signal. Both cases are measured with 5 $\mu \mathrm{N}$ in normal load and $0.25 \mathrm{~mm} / \mathrm{s}$ in shearing velocity.

It can be seen that even though the surface roughness affects the lateral forces, the "base line" of the lateral forces for both the $80{ }^{\circ} \mathrm{C}$ and $150{ }^{\circ} \mathrm{C}$ cases is clearly recognizable. The tribofilm formed at $150{ }^{\circ} \mathrm{C}$ presents a larger lateral force when being sheared, implying a higher shearing force than the tribofilm formed at $80^{\circ} \mathrm{C}$.

To calculate the shear strength Eq. (2-7), the Young's modulus of the tribofilms is taken from the results presented in Section 2.3.

The average results along the scanning areas and the standard deviations are plotted in Fig. 2.10. 


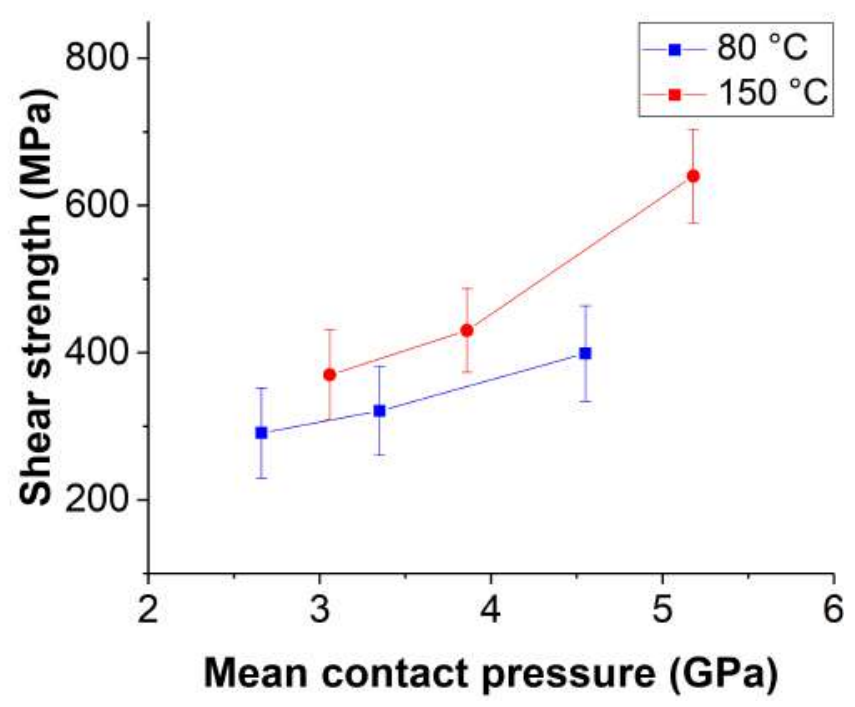

(a)

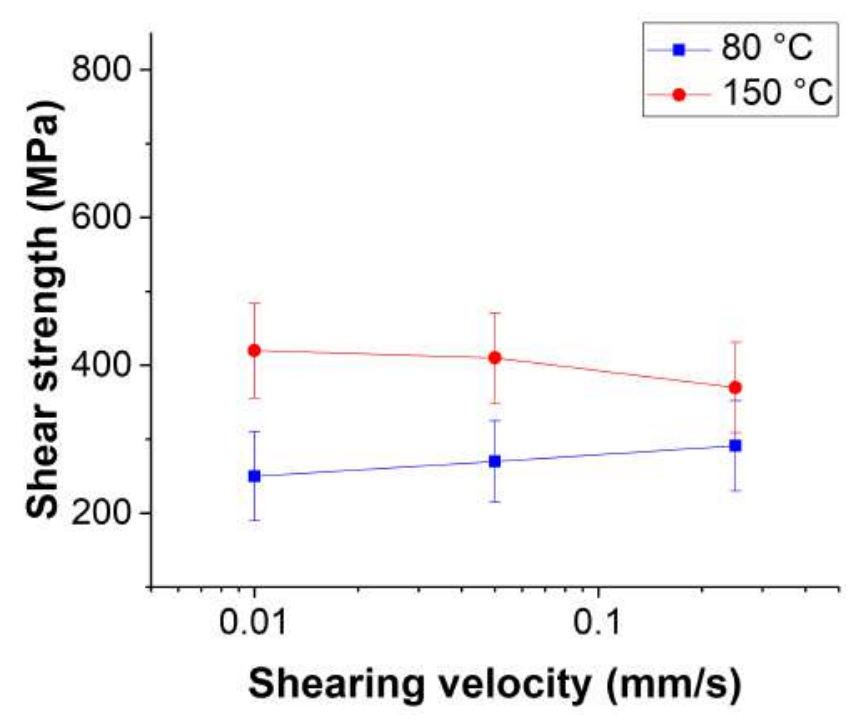

(b)

Fig. 2.10. AFM shear strength of the tribofilms under different test conditions: (a) Shear strength vs. mean contact pressure (with the shearing velocity of 0.25 $\mathrm{mm} / \mathrm{s}$ ) and (b) Shear strength vs. shearing velocity (with the normal load of $1 \mu \mathrm{N}$ ). 
In general, the results present noticeable standard deviations, which are caused by the surface roughness and the material inhomogeneity. However, the trends are rather clear. The tribofilms formed at $150{ }^{\circ} \mathrm{C}$ show a higher shearing strength than the $80^{\circ} \mathrm{C}$ cases under all testing conditions. This is probably because of the different chemical compositions, which are shown in Section 2.2. This higher shear strength may be connected to the higher coefficient of friction observed in Fig. 2.2.

In addition, it can be concluded that the shear strength of the tribofilm is affected by the average contact pressure, but affected very little by the shearing velocity in the range of $0.01 \mathrm{~mm} / \mathrm{s}$ to $0.25 \mathrm{~mm} / \mathrm{s}$.

\subsection{Summary}

In this chapter, pin-on-disc sliding tests lubricated by the fully formulated oil Shell NS-3 are carried out. The friction observed is linked to the morphology, thickness, chemical composition and mechanical properties of the tribofilm that are measured by a variety of characterizing techniques. The findings are summarized as follows.

(1) The tribosystem shows different friction levels when running at different temperatures. The coefficient of friction increases when the test temperatures are raised from $80^{\circ} \mathrm{C}$ to $150^{\circ} \mathrm{C}$.

(2) The tribofilms formed at $80^{\circ} \mathrm{C}$ and $150^{\circ} \mathrm{C}$ are similar in morphology and thickness but differ in chemical composition. The tribofilm formed at $150^{\circ} \mathrm{C}$ contains higher Fe content in all depths than the tribofilm formed at $80^{\circ} \mathrm{C}$. It is assumed that the chemical reactivity of the Fe substrate is higher at elevated temperatures.

(3) The tribofilms showed a noticeable difference in plastic (indentation) and elastic deformation. The film formed at $150{ }^{\circ} \mathrm{C}$ reveals high hardness ( $\left.4.7 \mathrm{GPa}\right)$ and Young's modulus ( $75 \mathrm{GPa}$ ), which is attributed to the high Fe content in the tribofilm formed at $150^{\circ} \mathrm{C}$. In contrast, the tested nanomechanical properties of film formed at $80{ }^{\circ} \mathrm{C}$ indicate a lower hardness value ( $43 \%$ ) and Young's modulus ( 25\%) than the tribofilm formed at $150^{\circ} \mathrm{C}$.

(4) Different shear strengths of the tribofilms formed at different temperatures are observed. The tribofilm formed at $150{ }^{\circ} \mathrm{C}$ shows higher shear strength than the counterpart at $80^{\circ} \mathrm{C}$. In addition, it is found that the shear strengths of the tribofilm 
are dependent on the mean contact pressure, but affected very little by the shearing velocity in the range of 0.01 to $0.25 \mathrm{~mm} / \mathrm{s}$.

(5) The different mechanical properties of the tribofilms formed at different temperatures may be linked to the higher Fe content in the tribofilm formed at $150{ }^{\circ} \mathrm{C}$. This also could be the reason for the higher coefficient of friction that is observed in the pin-on-disc test at $150{ }^{\circ} \mathrm{C}$.

\section{References}

[1] Larsson, P., Axen, N., \& Hogmark, S. (1999). Tribofilm formation on boron carbide in sliding wear. Wear, 236(1-2), 73-80.

[2] Kato, H., \& Komai, K. (2007). Tribofilm formation and mild wear by tribosintering of nanometer-sized oxide particles on rubbing steel surfaces. Wear, 262(1-2), 36-41.

[3] Equey, S., Roos, S., Mueller, U., Hauert, R., Spencer, N. D., \& Crockett, R. (2008). Tribofilm formation from ZnDTP on diamond-like carbon. Wear, 264(3-4), 316321.

[4] Tomala, A., Vengudusamy, B., Ripoll, M. R., Suarez, A. N., Remškar, M., \& Rosentsveig, R. (2015). Interaction between selected MoS 2 nanoparticles and ZDDP tribofilms. Tribology Letters, 59(1), 26.

[5] Zhang, Z., Yamaguchi, E. S., Kasrai, M., \& Bancroft, G. M. (2005). Tribofilms generated from ZDDP and DDP on steel surfaces: Part 1, growth, wear and morphology. Tribology Letters, 19(3), 211-220.

[6] Narita, K., \& Priest, M. (2009). Friction Characteristics and Topography of Tribofilms from Anti-Wear Additives Applied to Metal V-Belt Type CVT Fluids. Tribology Letters, 35(1), 45-56.

[7] Tomala, A., Vengudusamy, B., Ripoll, M. R., Suarez, A. N., Remškar, M., \& Rosentsveig, R. (2015). Interaction between selected MoS 2 nanoparticles and ZDDP tribofilms. Tribology Letters, 59(1), 26.

[8] Shimizu, Y., \& Spikes, H. A. (2016). The influence of slide-roll ratio on ZDDP tribofilm formation. Tribology Letters, 64(2), 19.

[9] Minfray, C., Martin, J. M., Esnouf, C., Le Mogne, T., Kersting, R., \& Hagenhoff, B. (2004). A multi-technique approach of tribofilm characterisation. Thin Solid Films, 447, 272-277. 
[10] Ito, K., Martin, J. M., Minfray, C., \& Kato, K. (2006). Low-friction tribofilm formed by the reaction of ZDDP on iron oxide. Tribology International, 39(12), 1538-1544.

[11] Qu, J., Meyer III, H. M., Cai, Z. B., Ma, C., \& Luo, H. (2015). Characterization of ZDDP and ionic liquid tribofilms on non-metallic coatings providing insights of tribofilm formation mechanisms. Wear, 332, 1273-1285.

[12] Dawczyk, J., Ware, E., Ardakani, M., Russo, J., \& Spikes, H. (2018). Use of FIB to study ZDDP tribofilms. Tribology Letters, 66(4), 155.

[13] Morina, A., Green, J. H., Neville, A., \& Priest, M. (2003). Surface and tribological characteristics of tribofilms formed in the boundary lubrication regime with application to internal combustion engines. Tribology Letters, 15(4), 443-452.

[14] Morina, A., \& Neville, A. (2007). Understanding the composition and low friction tribofilm formation/removal in boundary lubrication. Tribology International, 40(10-12), 1696-1704.

[15] Mourhatch, R., \& Aswath, P. B. (2009). Nanoscale properties of tribofilms formed with zinc dialkyl dithiophosphate (ZDDP) under extreme pressure condition. Journal of Nanoscience and Nanotechnology, 9(4), 2682-2691.

[16] Martin, J. M., Grossiord, C., Le Mogne, T., Bec, S., \& Tonck, A. (2001). The twolayer structure of Zndtp tribofilms: Part I: AES, XPS and XANES analyses. Tribology International, 34(8), 523-530.

[17] De Barros, M. I., Bouchet, J., Raoult, I., Le Mogne, T., Martin, J. M., Kasrai, M., \& Yamada, Y. (2003). Friction reduction by metal sulfides in boundary lubrication studied by XPS and XANES analyses. Wear, 254(9), 863-870.

[18] Ma, H., Li, J., Chen, H., Zuo, G., Yu, Y., Ren, T., \& Zhao, Y. (2009). XPS and XANES characteristics of tribofilms and thermal films generated by two P-and/or Scontaining additives in water-based lubricant. Tribology International, 42(6), 940-945.

[19] Carpick, R. W., \& Salmeron, M. (1997). Scratching the surface: Fundamental investigations of tribology with atomic force microscopy. Chemical Reviews, 97(4), 1163-1194.

[20] Mo, Y., Turner, K. T., \& Szlufarska, I. (2009). Friction laws at the nanoscale. Nature, 457(7233), 1116.

[21] Stachowiak, G., \& Batchelor, A. W. (2013). Engineering tribology. ButterworthHeinemann.

[22] Mortier, R. M., Orszulik, S. T., \& Fox, M. F. (Eds.). (2010). Chemistry and technology of lubricants (Vol. 107115). New York: Springer. 
[23] Tripaldi, G., Vettor, A., \& Spikes, H. (1996). Friction behaviour of ZDDP films in the mixed, boundary/EHD regime. SAE Transactions, 1819-1830.

[24] McQueen, J. S., Gao, H., Black, E. D., Gangopadhyay, A. K., \& Jensen, R. K. (2005). Friction and wear of tribofilms formed by zinc dialkyl dithiophosphate antiwear additive in low viscosity engine oils. Tribology International, 38(3), 289-297.

[25] Green, J. H., Morina, A., Priest, M., \& Neville, A. (2003). Evolution of tribofilms under lubrication conditions experienced in engine valve trains. In Tribology Series (Vol. 43, pp. 97-108). Elsevier.

[26] Morina, A., \& Neville, A. (2007). Tribofilms: aspects of formation, stability and removal. Journal of Physics D: Applied Physics, 40(18), 5476.

[27] Bec, S., Tonck, A., Georges, J. M., \& Roper, G. W. (2004). Synergistic effects of MoDTC and ZDTP on frictional behaviour of tribofilms at the nanometer scale. Tribology Letters, 17(4), 797-809.

[28] Aktary, M., McDermott, M. T., \& McAlpine, G. A. (2002). Morphology and nanomechanical properties of ZDDP antiwear films as a function of tribological contact time. Tribology Letters, 12(3), 155-162.

[29] Pereira, G., Munoz-Paniagua, D., Lachenwitzer, A., Kasrai, M., Norton, P. R., Capehart, T. W., ... \& Cheng, Y. T. (2007). A variable temperature mechanical analysis of ZDDP-derived antiwear films formed on 52100 steel. Wear, 262(34), 461-470.

[30] Pidduck, A. J., \& Smith, G. C. (1997). Scanning probe microscopy of automotive anti-wear films. Wear, 212(2), 254-264.

[31] Kano, M., Yasuda, Y., \& Ye, J. P. (2004). The effect of ZDDP and MoDTC additives in engine oil on the friction properties of DLC-coated and steel cam followers. Lubrication Science, 17(1), 95-103.

[32] Butt, H. J., Cappella, B., \& Kappl, M. (2005). Force measurements with the atomic force microscope: Technique, interpretation and applications. Surface Science Reports, 59(1-6), 1-152.

[33] Fischer-Cripps, A., \& Nicholson, D. (2004). Nanoindentation. Mechanical Engineering Series.

[34] Derjaguin, B. V., Muller, V. M., \& Toporov, Y. P. (1975). Effect of contact deformations on the adhesion of particles. Journal of Colloid and Interface Science, 53(2), 314-326.

[35] Gojzewski, H., Sadej, M., Andrzejewska, E., \& Kokowska, M. (2017). Nanoscale Young's modulus and surface morphology in photocurable polyacrylate/nanosilica composites. European Polymer Journal, 88, 205-220. 
[36] Wang, C., \& Schipper, D. (2018). On an elastoplastic sliding model for a coated single asperity. Lubricants, 6(4), 96.

[37] Pietrement, O., \& Troyon, M. (2001). Study of the interfacial shear strength pressure dependence by modulated lateral force microscopy. Langmuir, 17(21), 6540-6546.

[38] Liu, W., Bonin, K., \& Guthold, M. (2007). Easy and direct method for calibrating atomic force microscopy lateral force measurements. Review of Scientific Instruments, 78(6), 063707.

[39] Sader, J. E., Larson, I., Mulvaney, P., \& White, L. R. (1995). Method for the calibration of atomic force microscope cantilevers. Review of Scientific Instruments, 66(7), 3789-3798.

[40] Neumeister, J. M., \& Ducker, W. A. (1994). Lateral, normal, and longitudinal spring constants of atomic force microscopy cantilevers. Review of Scientific Instruments, 65(8), 2527-2531.

[41] Johnson, K. L., \& Johnson, K. L. (1987). Contact mechanics. Cambridge University Press.

[42] Nicholls, M. A., Norton, P. R., Bancroft, G. M., Kasrai, M., Do, T., Frazer, B. H., \& De Stasio, G. (2004). Nanometer scale chemomechanical characterization of antiwear films. Tribology Letters, 17(2), 205-216.

[43] Nicholls, M. A., Do, T., Norton, P. R., Kasrai, M., \& Bancroft, G. M. (2005). Review of the lubrication of metallic surfaces by zinc dialkyl-dithiophosphates. Tribology International, 38(1), 15-39. 


\section{A single asperity sliding friction model}

In this chapter, a single asperity sliding friction model based on a semi-analytical method is developed, to calculate the friction caused by deformation force with surface adhesion force being ignored. Firstly, the modelling approach is introduced, after which the model is verified by finite element analysis. Finally, the developed model is used to investigate the effects of interference depths and tribofilm properties on the single asperity sliding friction.

\subsection{Introduction}

In boundary lubrication, the film thickness is not sufficient to separate the asperities. Therefore, the asperities are carrying the load locally. On microscale level, plastic deformation may occur while the macroscopic pressure is still well below the yield limit. This elasto-plastic deformation of asperities will lead to energy dissipation. It has been regarded as one of the important sources of friction [1].

The finite element method (FEM) is a commonly used approach to solving elastoplastic single asperity interactions. Jackson et al. [2] developed a finite element model predicting the normal and tangential resisting forces of two sliding elastic, perfectly plastic frictionless spherical asperities. Mulvihill et al. [3] improved this work by imposing greater interference depths and considering surface adhesion in the contact. However, it should be noted that performing FEM contact analysis is time-consuming since a very fine mesh is needed in contact areas to ensure convergence and accurate results, while the domain needs to be large enough to avoid edge effects. In addition, the contact condition itself (no penetration of the two bodies) is not inherently incorporated in the FEM method and requires additional degrees of freedom (DOF) for a solution to be reached. Often, the penalty function is used, in which the magnitude of the contact stiffness is tuned using a Hertzian reference contact. While the obtained value may be true for the reference case, it is very possible that in different scenarios the values obtained do not hold.

The fast Fourier transform (FFT) technique and semi-analytical method (SAM) provide an alternative for solving elastic and elastoplastic contact more efficiently. Various problems including single and layered material contact have been solved by SAM [4]. Also, techniques have been developed to accelerate the calculation and 
improve the accuracy. Polonsky and Keer [5] proposed an iterative scheme by applying the conjugate gradient method. Liu et al. [6] eliminated the periodic errors in regular FFT by introducing the discrete fast Fourier transform (DC-FFT) technique. However, the aforementioned works only handled purely elastic contacts. To solve elastoplastic contact problems, Jacq et al. [7] presented a semi-analytical method by considering plastic deformation as eigenstrains, based on Chiu's results [8][9]. Recently, Wang et al. [10] acquired stress and displacement fields of eigenstrains in two perfectly bonded layered half-spaces, making it possible to solve the elastoplastic contact of layered material.

In this Chapter, the friction caused by elasto-plastic deformation of asperities are solved by applying the DC-FFT technique and SAM. Consequently, this work extends the model proposed by Boucly et al. [11], which involved only homogeneous material, to a layered system. The plasticity in the layered structure is considered to follow the $\mathrm{J} 2$ flow theory and is solved by the method in reference [12]. Green's assumption on steady state sliding [13] is applied: two asperities will not approach or separate over each other while sliding, a predefined separation of the two surfaces is given. The adhesion between tribofilms and damage to asperities are ignored, as the main goal of this chapter is to investigate the contribution of elasto-plastic deformation of asperities to friction.

\subsection{Development of single asperity sliding model}

In this part, a single asperity sliding model is developed to calculate the friction caused by asperity deformation in the pin-on-disc tribosystem discussed in Chapter 2. The model setup is shown in Fig. 3.1. Two single asperities covered with a tribofilm are considered. The radius of the asperities is respectively $\boldsymbol{R}_{\mathbf{1}}$ and $\boldsymbol{R}_{\mathbf{2}}$, with an interference depth $\boldsymbol{\delta}$. The upper asperity is sliding, while the bottom one is set to be fixed. During the sliding process, two asperities interact and a tangential force is needed to maintain the sliding. This force is regarded as the friction force and should be predicted by the model to be developed. 


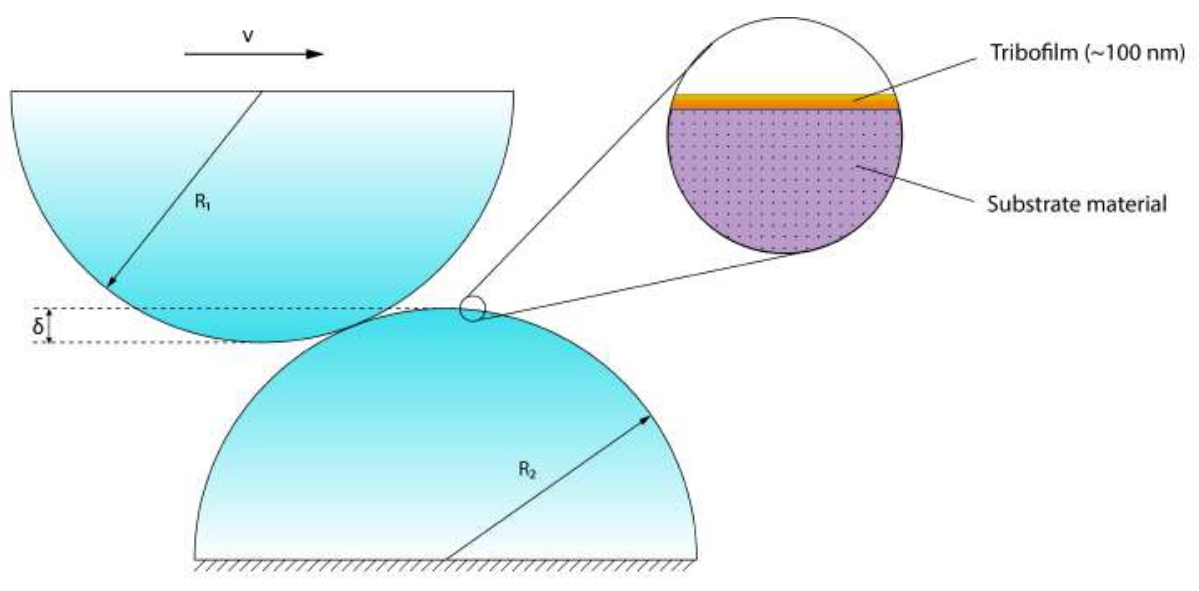

Fig. 3.1. Schematic of the single asperity sliding model.

To achieve this goal, first a displacement-driven contact model is developed in Subsection 3.2.1 as the tool, then the friction forces are calculated based on the displacement-driven contact model together with inputs derived from the geometry relationships, which are introduced in Subsection 3.2.2.

\subsubsection{Displacement-driven contact model}

The schematic of the displacement-driven model is presented in Fig. 3.2. For simplicity but without loss of generality, an elasto-plastic object indenting on a rigid flat is considered. The geometry information of the object is depicted by $\boldsymbol{h}_{\mathbf{0}}$, which is the initial surface separation without deformation. Then a rigid body movement $\boldsymbol{\delta}$ is put on the object and the contact occurs. A contact pressure distribution $\boldsymbol{p}$ will be built, with plastic strains $\boldsymbol{e}_{\boldsymbol{p}}$ being generated. Due to the contact pressure and the plastic strains, elastic and plastic deformations $\left(\boldsymbol{u}_{\boldsymbol{e}}\right.$ and $\left.\boldsymbol{u}_{\boldsymbol{r}}\right)$ occur. 


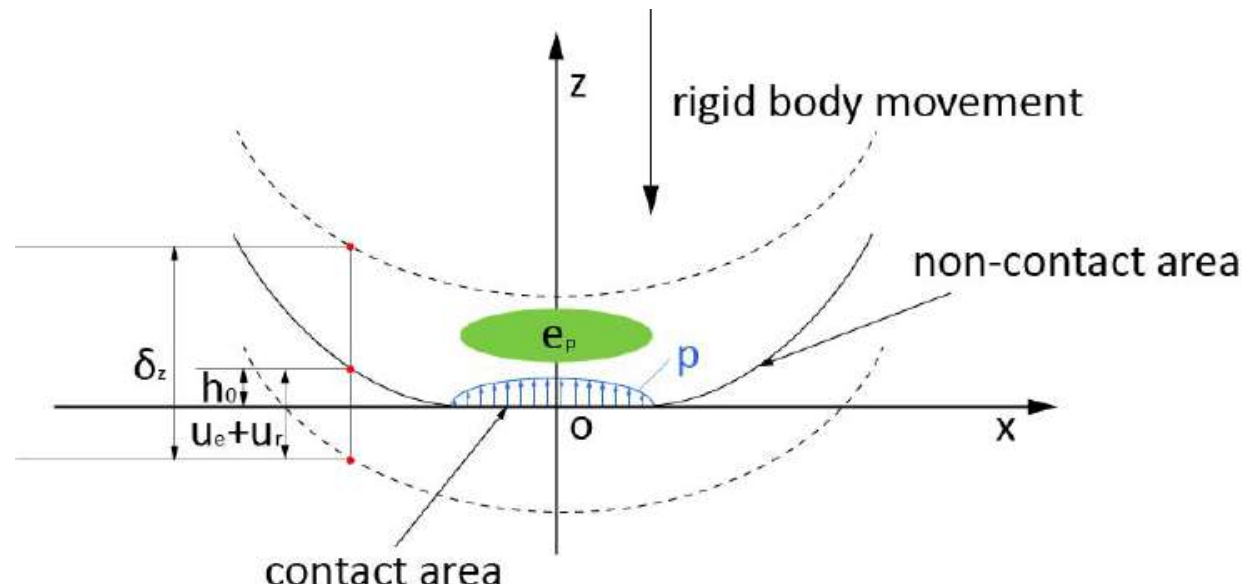

Fig. 3.2. Geometry of the surface contact.

For this displacement-driven contact model, the rigid body displacement $(\boldsymbol{\delta})$, together with the geometry information of the object $\left(\boldsymbol{h}_{\mathbf{0}}\right)$ and its mechanical properties, serve as input for the model. The model should be able to predict the contact pressure $(\boldsymbol{p})$, so that the total contact force can be further calculated as:

$$
\boldsymbol{F}_{\text {contact }}=\sum \boldsymbol{p} * d \boldsymbol{A}
$$

To achieve this objective, the geometry of the elasto-plastic object after contact deformation is considered. Notice that in the whole domain in which contact and noncontact areas both exist, the following equations and conditions hold:

Inside the contact area $(\boldsymbol{p}>\mathbf{0})$ :

$$
u_{e}(p)+u_{r}\left(e_{p}\right)+h_{0}=\delta_{z}
$$

Outside the contact area $(\boldsymbol{p}=\mathbf{0})$ : 


$$
u_{e}(p)+u_{r}\left(e_{p}\right)+h_{0} \geq \delta_{z}
$$

Eq. (3-2) and (3-3) indicate that in the contact area the surface separation must be zero (no penetration). In the non-contact areas, however, the surface separation should be greater than zero. The detailed calculation procedures of $\boldsymbol{u}_{\boldsymbol{e}}$ and $\boldsymbol{u}_{r}$ are listed in Appendix 1.

Now, the pressure distribution $\boldsymbol{p}$ and plastic strains $\boldsymbol{e}_{\boldsymbol{p}}$ are explicitly linked to the inputs by Eq. (3-2) and (3-3). This is in essence a solvable $M^{*} N$ linear system. However, it should be noted that the subsurface stress generated by the surface contact pressure causes plastic strains. These plastic strains cause residual displacement and stresses, which in turn alter the geometry and change the surface contact pressure. In other words, $\boldsymbol{p}$ and $\boldsymbol{e}_{\boldsymbol{p}}$ are coupled. Thus, a direct solution is difficult to acquire.

An iteration loop is proposed to address the problem. First, an initial status is set. The pressure and subsurface stress are solved first, followed by a radial return algorithm to determine plastic deformation for the current loop. Then, the residual displacement is calculated and surface geometry is updated. Afterwards, the pressure distribution is recalculated based on the new surface geometry. This loop will keep running till a convergence in pressure distribution is achieved. Note that in each iteration, an inner iteration (return mapping) is needed to map the stress back to yield surface and update plastic deformation. This process is shown in Fig. $\mathbf{3 . 3}$ and Fig. 3.4. 


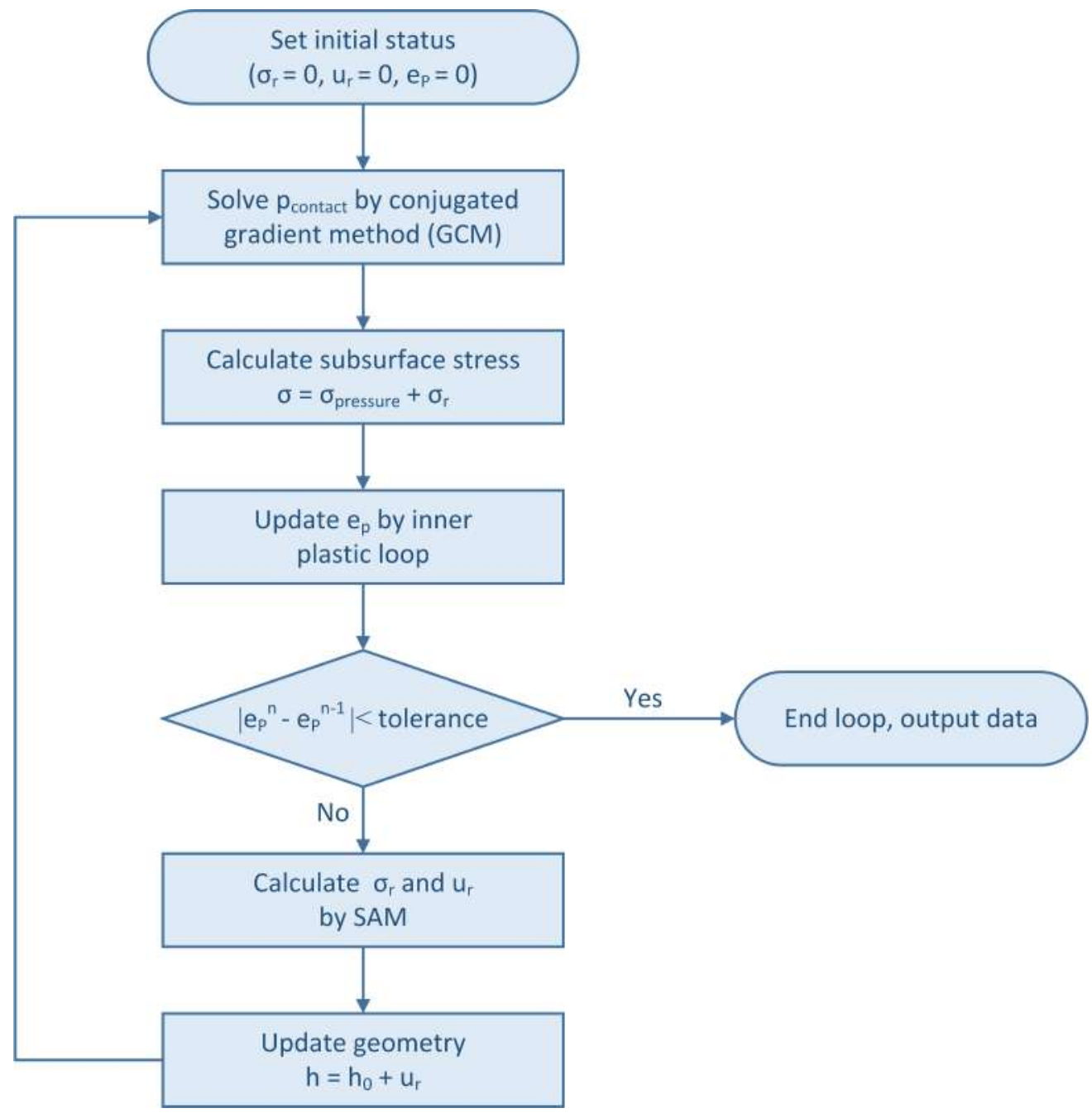

Fig. 3.3. Global iteration loop. 


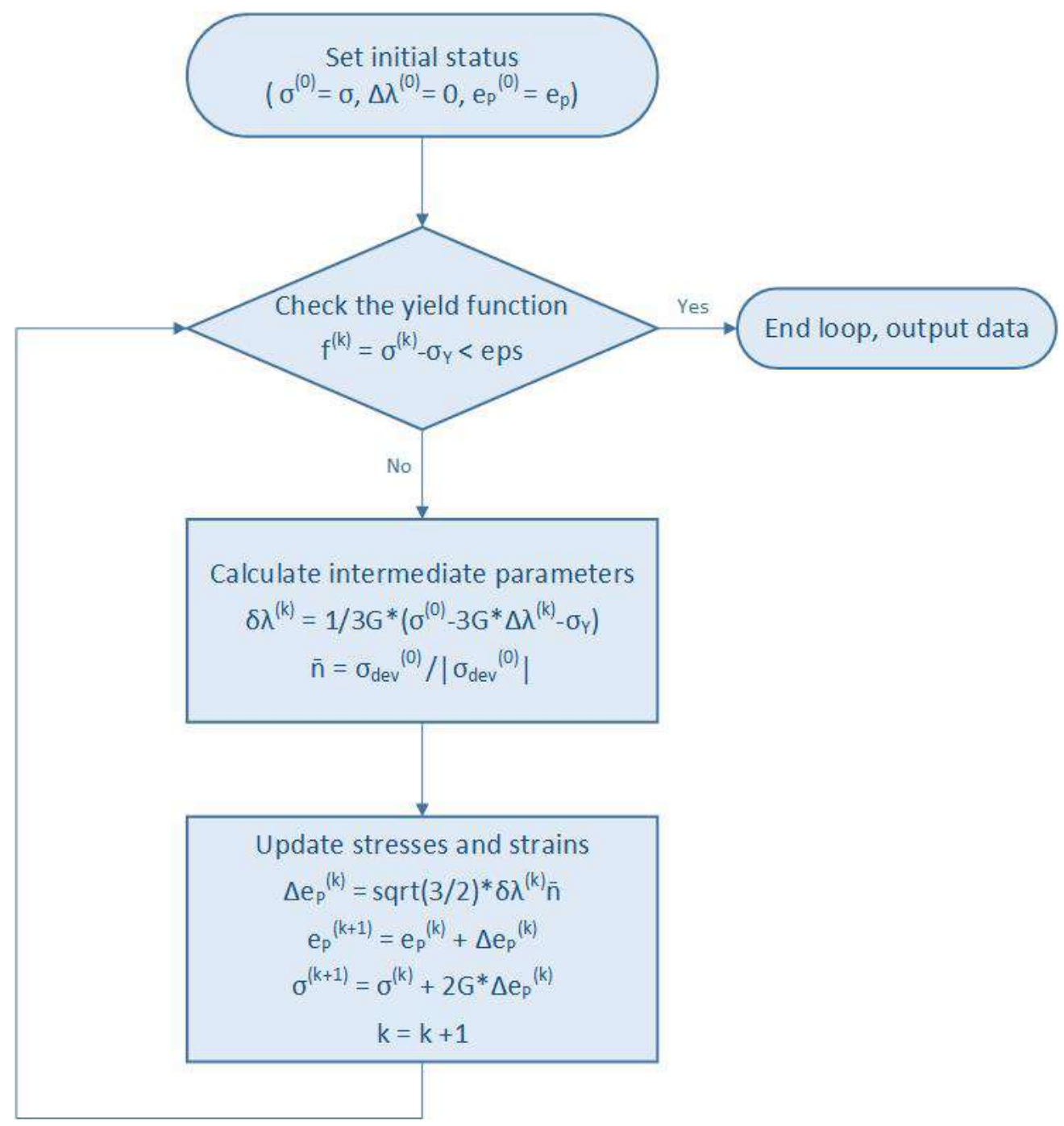

Fig. 3.4. Inner iteration loop for plasticity.

3.2.2 Single asperity sliding model

With the displacement-drive contact model ready as the tool, the asperity sliding model can be further developed. The model setup is shown in Fig. 3.5. During the whole sliding process, a contact force $\boldsymbol{F}_{\text {contact }}$ will be generated with corresponding contact pressure distribution. This force can be split into two perpendicular 
components $\boldsymbol{F}_{\boldsymbol{x}}$ and $\boldsymbol{F}_{\boldsymbol{z}}$, which can be regarded as the normal force and friction force. The single asperity sliding model should be able to calculate all these three forces.

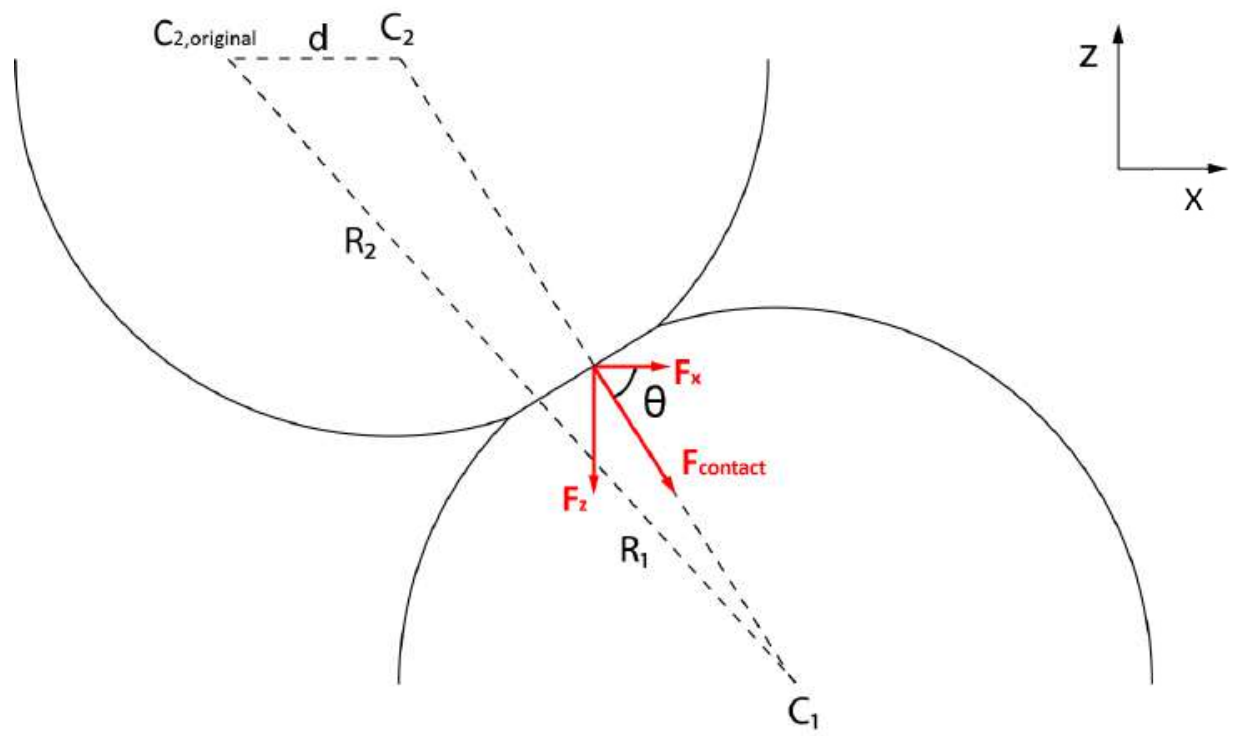

Fig. 3.5. Contact force of asperity contact.

Notice that these three forces are built on the basis of the geometry interaction of the two single asperities. At any sliding position, there is a certain interference depth. Therefore, the previously developed displacement-driven model can be applied to calculate $\boldsymbol{F}_{\text {contact }}$ if the interference force is known. Then $\boldsymbol{F}_{\boldsymbol{x}}$ and $\boldsymbol{F}_{\boldsymbol{z}}$ can be further calculated based on the geometry layout.

This problem is dealt with by discretization. The whole sliding contact process is discretized into $n$ equally distributed incremental steps. In the $i^{\text {th }}$ step, observe the triangle $\boldsymbol{C}_{2, \text { original }}-\boldsymbol{C}_{\mathbf{1}}-\boldsymbol{C}_{\mathbf{2}}, \boldsymbol{d}$ is the sliding distance of the upper asperity from its original position, and $\boldsymbol{C}_{\mathbf{1}} \boldsymbol{C}_{\mathbf{2}}$ is the distance between the two centers of the asperities, which can be calculated easily by the law of Cosines. After that, the interference depth can be calculated as

$$
\delta_{i}=R_{1}+R_{2}-C_{1} C_{2, i}
$$


With $\boldsymbol{\delta}_{\boldsymbol{i}}$ calculated, the contact force in each step $\boldsymbol{F}_{\text {contact, } i}$ can be calculated by the displacement-driven contact model. The frictional force and normal force in each step then can be further calculated as:

$$
\begin{aligned}
& F_{x, i}=F_{\text {contact }, i} * \cos \theta \\
& F_{z, i}=F_{\text {contact }, i} * \sin \theta
\end{aligned}
$$

where $\theta$ is the angle between $\boldsymbol{F}_{\text {contact }}$ and the horizontal plane.

For the whole sliding process, the coefficient of friction (COF) is defined as

$$
\operatorname{COF}=\frac{\sum_{i=1}^{n} F_{x, i}}{\sum_{i=1}^{n} F_{z, i}}
$$

Readers are referred to Boucly's work [11] for detailed expressions.

\subsection{FEM verification of displacement-driven contact model}

The displacement-driven contact model proposed in Section $\mathbf{3 . 2}$ is verified by an asperity indented on a coated flat plane. For simplicity without losing generality, the asperity is assumed rigid, while both materials of the coated flat plane showed elastic, perfectly plastic behaviour. The material properties and contact conditions are listed below in Tab. 3.1. Two different yield strengths of the coating material are used for comparison. The solution domain is meshed into an $80 \times 80 \times 80$ grid (20 grid over the thickness direction of tribofilm). With a single element of size $6 \times 6 \times 6$ $\mathrm{nm}$, this resulted a calculation domain sized $480 \times 480 \times 480 \mathrm{~nm}$, which is large enough to allow the plastic deformation to extend fully. 
Tab. 3.1. Parameters of the indentation model.

\begin{tabular}{cc}
\hline Parameter & Value \\
\hline Young's modulus, $\mathrm{E}_{\text {coating }}(\mathrm{GPa})$ & 35 \\
Poisson's ratio, $\mathrm{v}_{\text {coating }}$ & 0.3 \\
Yield stress, $\sigma_{\text {coating }}(\mathrm{MPa})$ & 500,800 \\
Young's modulus, $\mathrm{E}_{\text {substrate }}(\mathrm{GPa})$ & 205 \\
Poisson's ratio, $\mathrm{v}_{\text {substrate }}$ & 0.3 \\
Yield stress, $\sigma_{\text {substrate }}(\mathrm{MPa})$ & 1200 \\
Radius of asperity $(\mu \mathrm{m})$ & 10 \\
Indentation depth $(\mathrm{nm})$ & 10 \\
Thickness of film, $\mathrm{h}_{\text {film }}(\mathrm{nm})$ & 120 \\
\hline
\end{tabular}

The results of the SAM model are verified by comparing the solutions with those from commercial FEM software ABAQUS. The FEM model used four-node bilinear axisymmetric quadrilateral with full integration (type CAX4) elements. The element sizes varied from near the contact area (indicating the volume which has this fine mesh, $6 \times 6 \mathrm{~nm}$ ) to $100 \times 100 \mathrm{~nm}$ in far-away areas. To limit the effect of boundary conditions in the FEM simulation, the modelling domain had to be at least ten times larger than the contact zone. This gives a total of 55,214 elements in the FEM model.

A comparison of the results is presented in Fig. 3.6. The von Mises stress and equivalent plastic strain as a function of depth at the centre of the contact are shown. Despite some minor differences, good agreements are observed. 


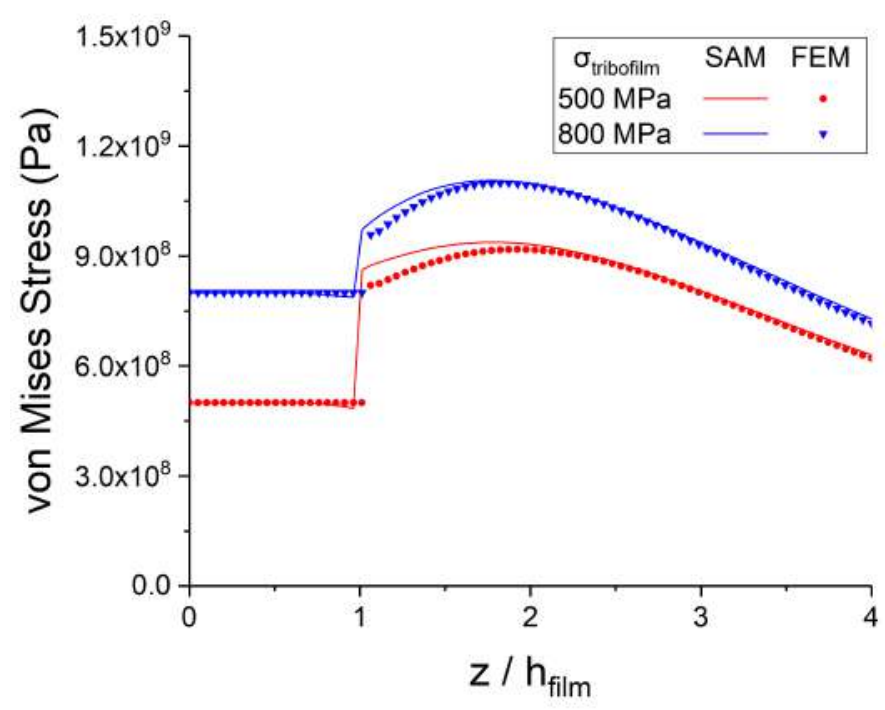

(a)

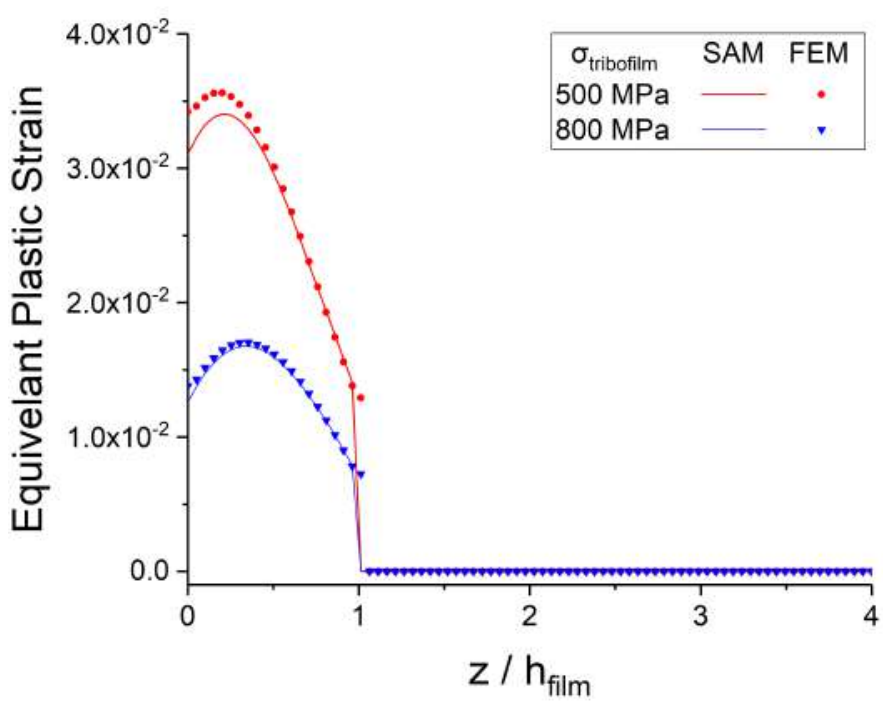

(b)

Fig. 3.6. Comparison of finite element method (FEM) and semi-analytical method (SAM) results along the $z$ axis $(x=0, y=0)$ : (a) von Mises stress, and (b) equivalent plastic strain. 
Contour plots of the von Mises stresses and equivalent plastic strain in the $x-z$ plane are shown in Fig. 3.7. The results show that plastic strains solely occur in the tribofilm due to its lower yield strength than steel. For the $500 \mathrm{MPa}$ case, the plastic strain tends to concentrate near the bonding interface, while for the $800 \mathrm{MPa}$ case, the plastic strain is more homogeneously distributed.

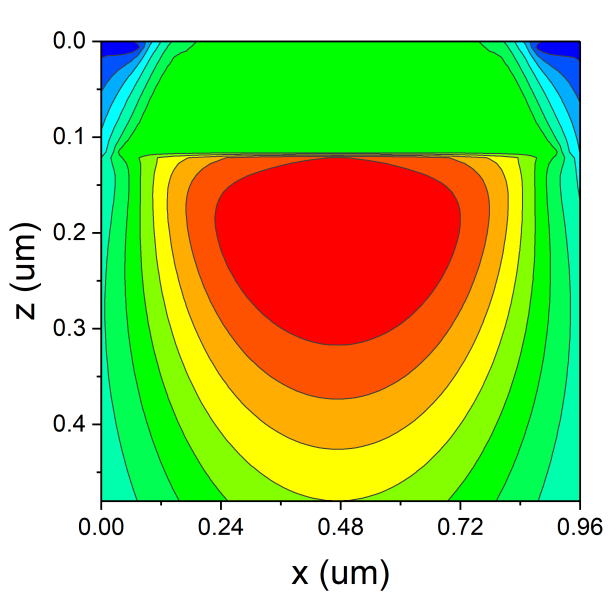

Mises Stress

(a)

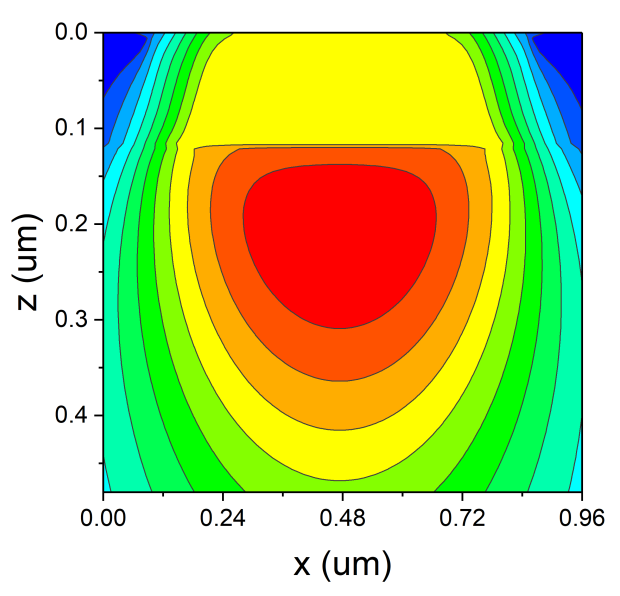

Mises Stress
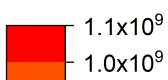

$-9.3 \times 10^{8}$

$-8.4 \times 10^{8}$

$-7.5 \times 10^{8}$

$-6.6 \times 10^{8}$

$-5.7 \times 10^{8}$

$-4.8 \times 10^{8}$

$-3.9 \times 10^{8}$

$3.0 \times 10^{8}$

$2.1 \times 10^{8}$

$-1.2 \times 10^{8}$

$2.5 \times 10^{7}$

(b)

Fig. 3.7. Contour of von Mises stress: (a) $\sigma_{\text {tribofilm }}=500 \mathrm{MPa}$ and (b) $\sigma_{\text {tribofilm }}=800$ $\mathrm{MPa}$. 


\section{Plastic Strain}
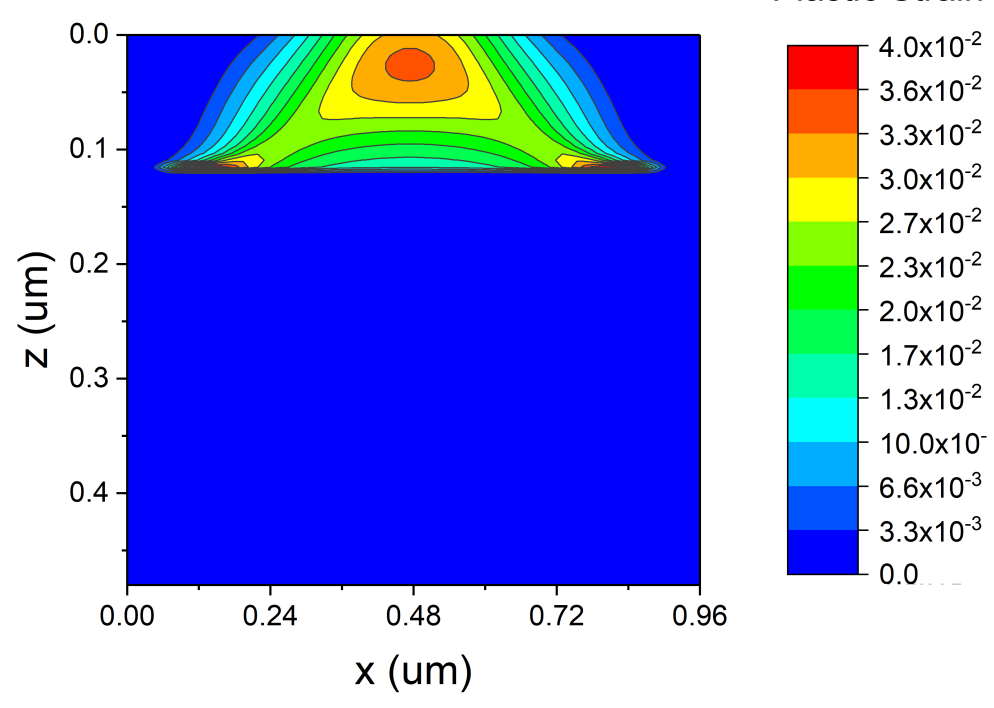

(a)

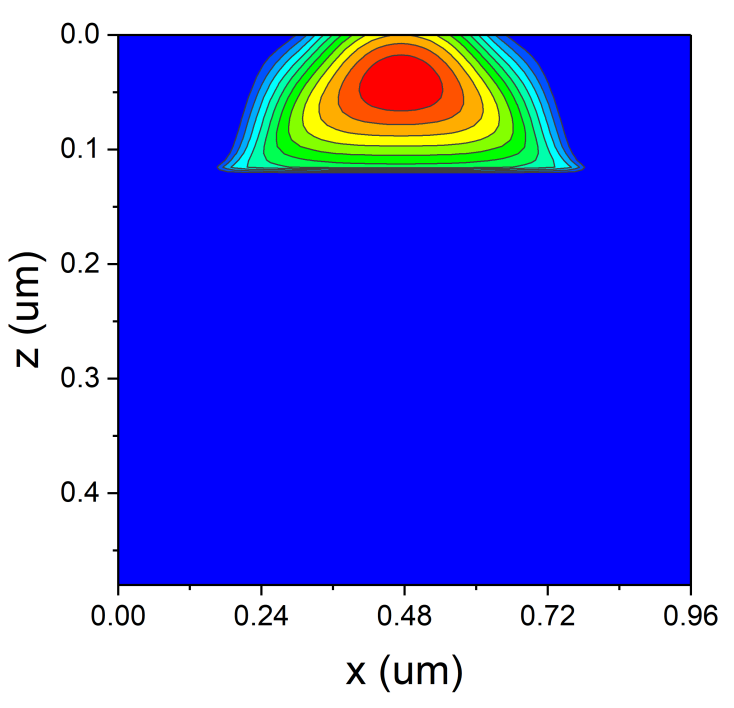

Plastic Strain

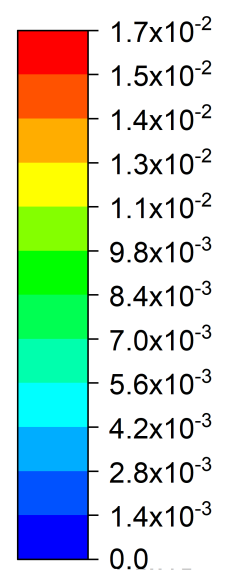

(b)

Fig. 3.8. Contour of equivalent plastic strain: (a) $\sigma_{\text {tribofilm }}=500 \mathrm{MPa}$ and (b) $\sigma_{\text {tribofilm }}$ $=800 \mathrm{MPa}$. 


\subsection{Effects of interference depth and tribofilm properties on sliding friction}

In this part, the aforementioned single sliding model is applied to quantify the effects of interference depths and tribofilm properties on the sliding friction caused by asperity deformation, with the adhesion effect being ignored.

\subsubsection{Effect of elastic modulus of tribofilm}

A series of interference depths are applied (20,35, 50, and $65 \mathrm{~nm}$ ), while other parameters are kept the same as those listed in Tab 3.1. The results are shown in Fig. 3.9, where it becomes clear that the tangential force increases with interference depth, which makes sense as more material is plastically deformed. This is also reflected by the increase in calculated coefficient of friction (Fig. 3.9c).

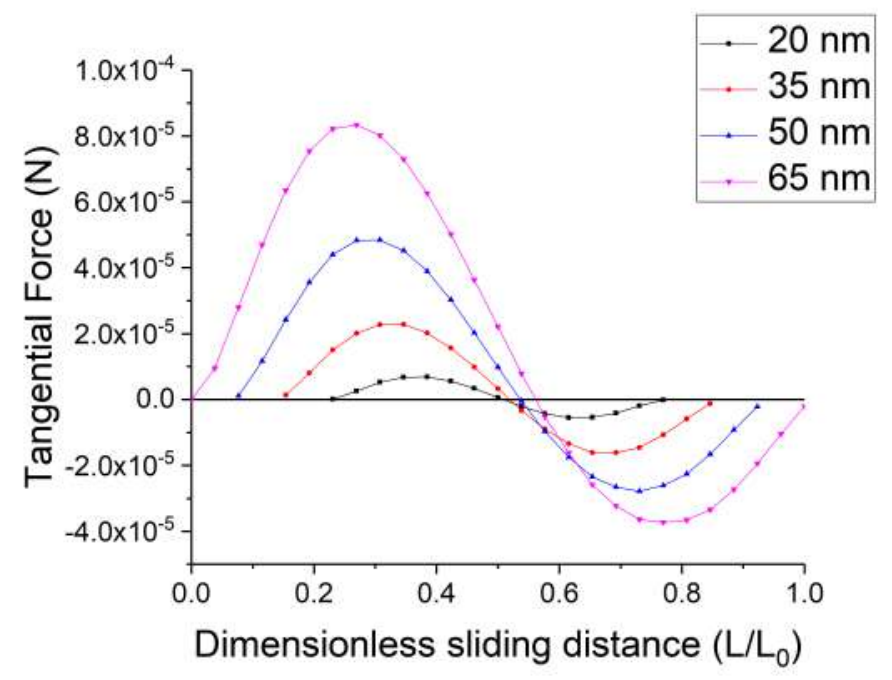

(a) 


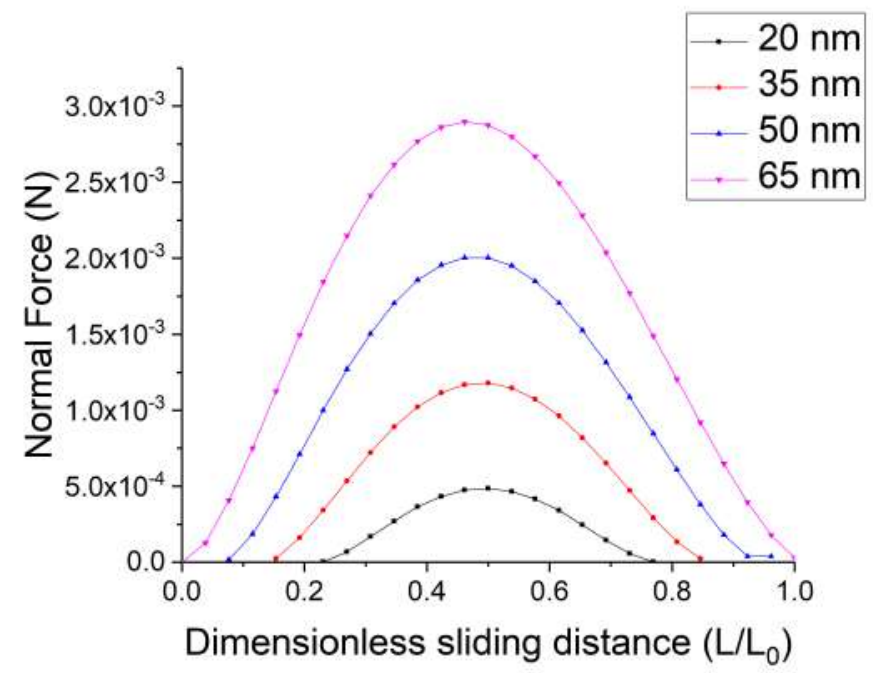

(b)

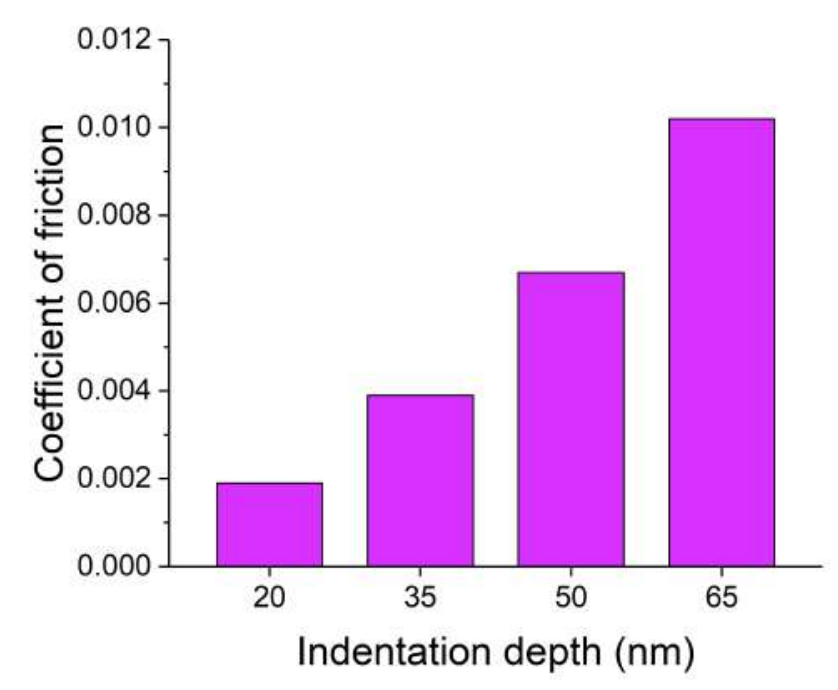

(c)

Fig. 3.9. Results for different indentation depths: (a) frictional force; (b) normal force and (c) coefficient of friction.

3.4.2 Effect of elastic modulus of tribofilm 
The elastic modulus of the tribofilms are chosen as 20, 35, 50 and $65 \mathrm{GPa}$. Other material properties are kept the same as those listed in Tab. 3.1. The frictional and normal forces are shown in Fig. 3.10.

The results show that the frictional and normal forces increase with a stiffer tribofilm than expected. Also, a tribofilm with higher elastic modulus leads to a higher coefficient of friction. This is because with a higher elastic modulus the tribofilm reaches its elastic limit at a lower strain level, which leads to a greater volume of plasticity. Additionally, a higher elastic modulus means more energy is needed for the same amount of plastic deformation. So for these two reasons, more energy is absorbed by the system, thus generating greater friction.

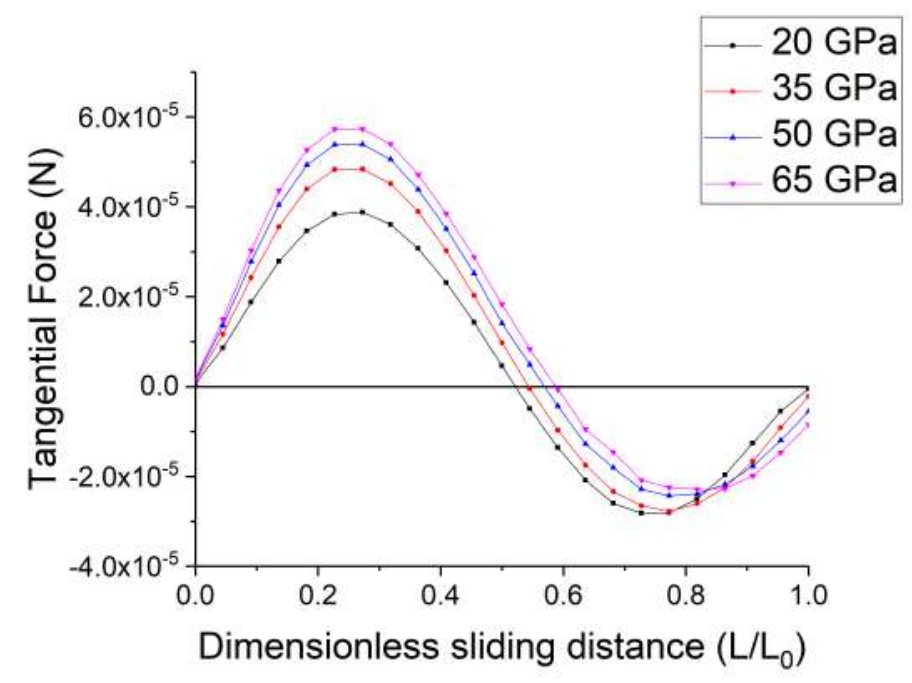

(a) 


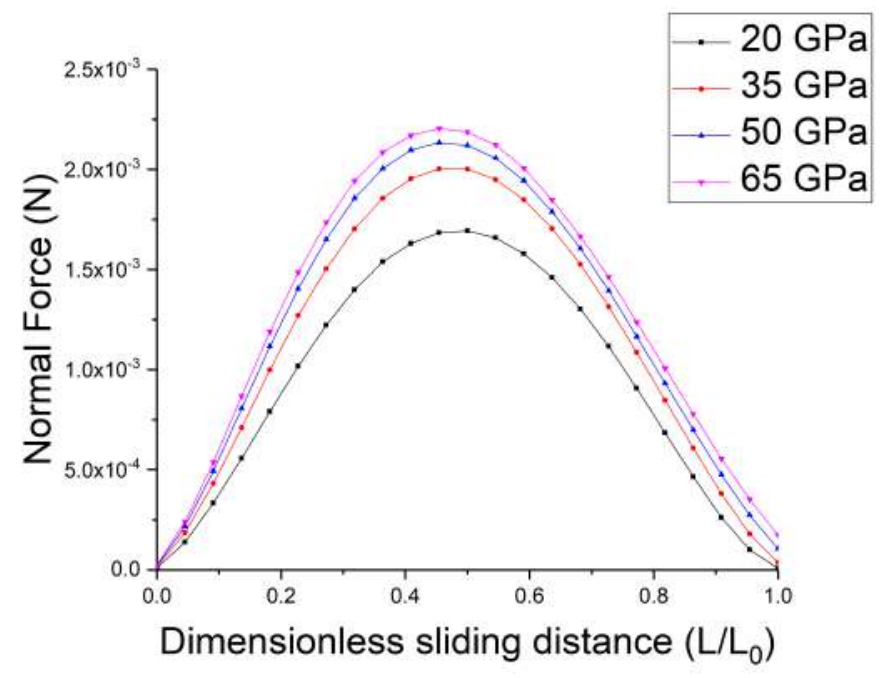

(b)

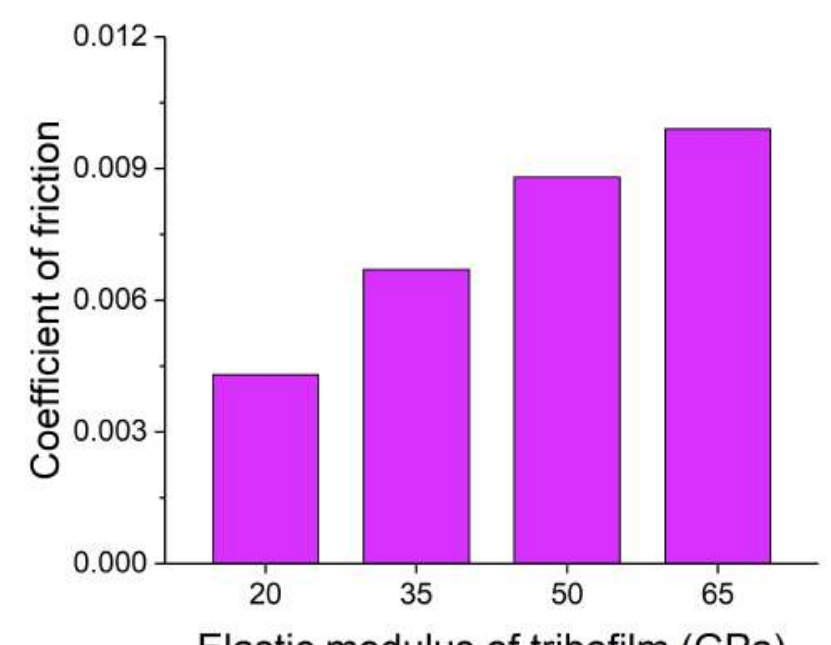

Elastic modulus of tribofilm (GPa)

(c)

Fig. 3.10. Results for different tribofilm elastic modulus: (a) frictional force; (b) normal force and (c) coefficient of friction.

3.4.3 Effect of yield strength of tribofilm 
In this part, the effect of the yield stress of the tribofilm on sliding friction is quantified. The yield stresses of the tribofilms are set as 350, 500, 650 and $800 \mathrm{MPa}$. Other material properties are kept the same as those listed in Tab. 3.1.

The results are shown in Fig. 3.11.

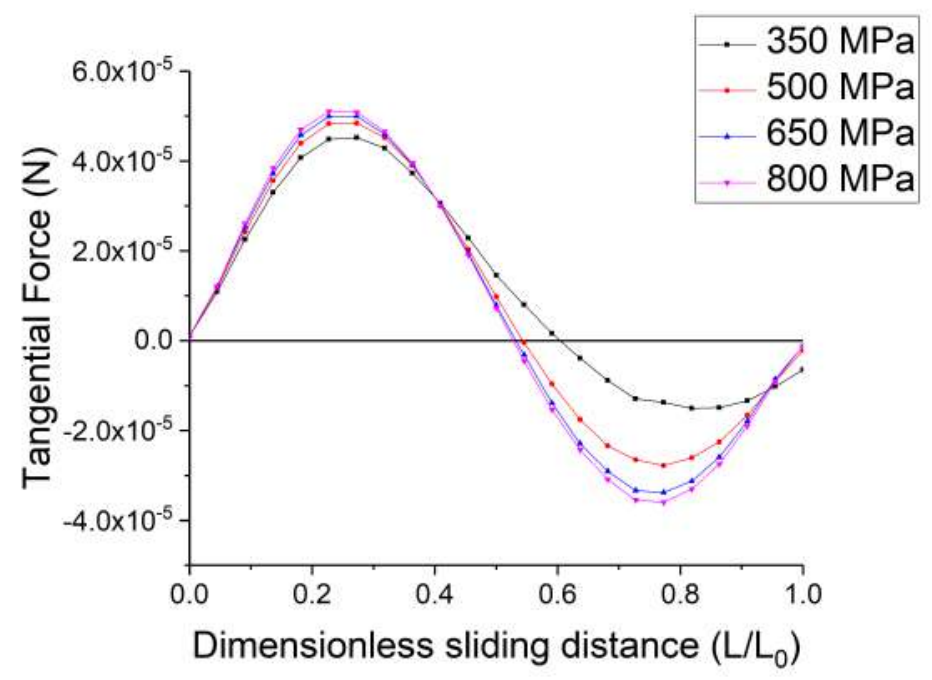

(a)

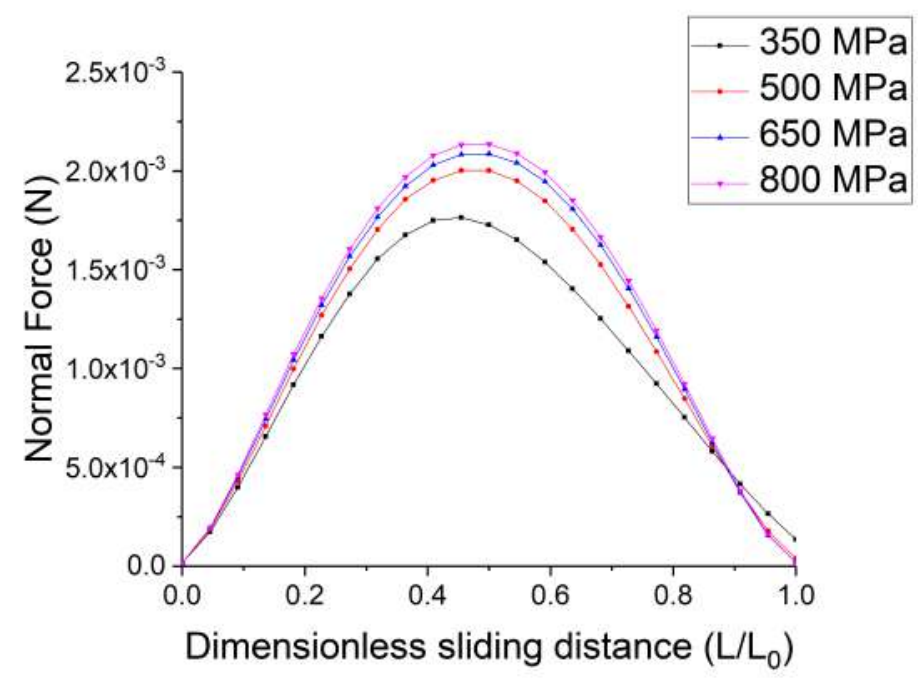

(b) 


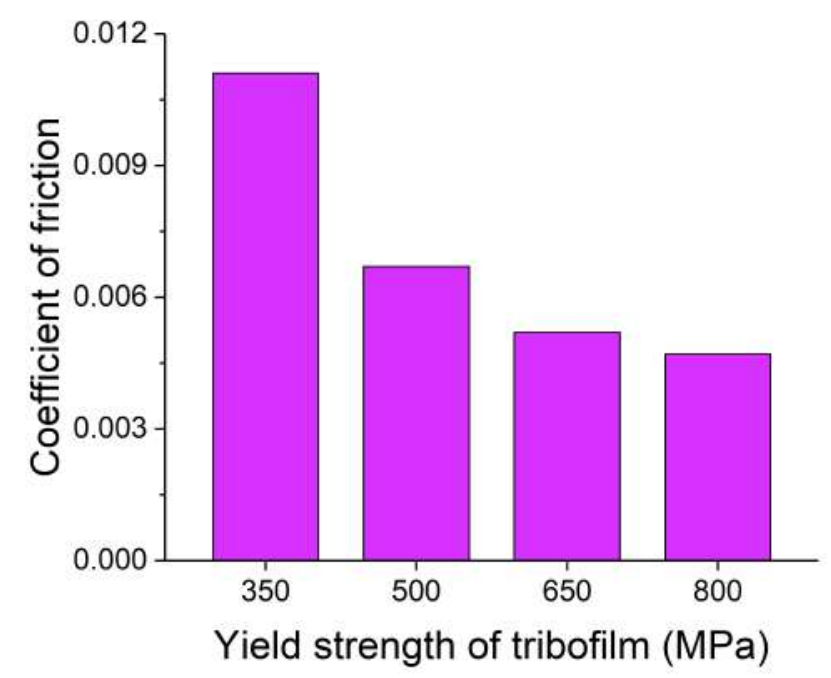

(c)

Fig. 3.11. Results for different tribofilm yield strengths: (a) frictional force; (b) normal force and (c) coefficient of friction.

From the results, it can be concluded that a tribofilm with a higher yield strength will cause a higher normal force, which is due to less plastic strain/displacement and thus a smaller contact area. Also, the coefficient of friction will decrease due to a higher yield strength of the tribofilm resulting from less plastic deformation. Therefore, the system will be affected less and show lower friction.

\subsection{Summary}

In this chapter, a SAM based single asperity sliding model is developed. This model is able to predict the tangential and normal forces due to contact during sliding. The indentation model is verified by FEM and good agreement is achieved.

The single asperity sliding model is applied to investigate the effects of tribofilm properties. The results suggest that with all other parameters fixed, larger interference depth, greater elastic modulus, or lower yield strength of the tribofilm will lead to higher sliding friction. 


\section{References}

[1] Komvopoulos, K., Saka, N., and Suh, N. P. (1985). The mechanism of friction in boundary lubrication. Journal of Tribology, 107(4), 452-462.

[2] Jackson, R. L., Duvvuru, R. S., Meghani, H., \& Mahajan, M. (2007). An analysis of elasto-plastic sliding spherical asperity interaction. Wear, 262(1-2), 210-219.

[3] Mulvihill, D. M., Kartal, M. E., Nowell, D., \& Hills, D. A. (2011). An elastic-plastic asperity interaction model for sliding friction. Tribology International, 44(12), 1679-1694.

[4] Ju, Y., and Farris, T. N. (1996). Spectral analysis of two-dimensional contact problems. Journal of Tribology, 118(2), 320-328.

[5] Polonsky, I. A., \& Keer, L. M. (1999). A numerical method for solving rough contact problems based on the multi-level multi-summation and conjugate gradient techniques. Wear, 231(2), 206-219.

[6] Liu, S., Wang, Q., \& Liu, G. (2000). A versatile method of discrete convolution and FFT (DC-FFT) for contact analyses. Wear, 243(1-2), 101-111.

[7] Jacq, C., Ne' lias, D., Lormand, G., \& Girodin, D. (2002). Development of a three-dimensional semi-analytical elastic-plastic contact code. Journal of Tribology, 124(4), 653-667.

[8] Chiu, Y. P. (1977). On the stress field due to initial strains in a cuboid surrounded by an infinite elastic space. Journal of Tribology, 44(4), 587-590.

[9] Chiu, Y. P. (1978). "On the stress field and surface deformation in a half space with a cuboidal zone in which initial strains are uniform. Journal of Tribology, 45(2), 302-306.

[10] Wang, Z., Yu, H., \& Wang, Q. (2016). Analytical solutions for elastic fields caused by eigenstrains in two joined and perfectly bonded half-spaces and related problems. International Journal of Plasticity, 76, 1-28.

[11] Boucly, V., Nélias D., \& Green, I. (2007). Modeling of the rolling and sliding contact between two asperities. Journal of Tribology, 129(2), 235-245.

[12] Simo, J. C., \& Taylor, R. L. (1985). Consistent tangent operators for rateindependent elastoplasticity. Computer Methods in Applied Mechanics and Engineering, 48(1), 101-118.

[13] Green, A. P. (1955). Friction between unlubricated metals: a theoretical analysis of the junction model. Proceedings of the Royal Society of London. Series A. Mathematical and Physical Sciences, 228(1173), 191-204. 


\section{Real contact area in rough surface contact}

In this chapter, the real contact area of interacting rough surfaces with predefined normal loads are investigated, for further calculation of the adhesive force. A semianalytical based, elastic, perfectly plastic model is proposed as numerical tool. The sensitivity of the grid size of inputs to the calculated real contact areas is studied and an extrapolation method is proposed for more accurate results.

\subsection{Introduction}

As discussed in Chapter 1, in boundary lubrication the normal load will be carried by the asperities coming into contact. The sum of the area in contact is referred to as real contact area $\left(\boldsymbol{A}_{\boldsymbol{r}}\right)$. It is common that the real contact area is only a small percentage of the nominal contact area. Therefore, the local contact load in real contact areas can be high and cause adhesive bonds.

When the two contacting surfaces slide along each other, the local adhesive bonds must be separated in order to accommodate the relative motion. A lateral force is therefore needed, which is regarded as the friction caused by adhesive force. This force is written as Eq. (1-3):

$$
\boldsymbol{F}_{\boldsymbol{a}}=\boldsymbol{\tau} * \boldsymbol{A}_{\boldsymbol{r}}
$$

The shear strength $\boldsymbol{\tau}$ has already been measured by LFM (see Chapter 2 ). To further determine the adhesive force, the real contact area $\left(\boldsymbol{A}_{\boldsymbol{r}}\right)$ is required.

The semi-analytical method (SAM) provides an effective approach in numerically solving the real contact area. However, as a numerical method, SAM requires discretized surface geometry data as input. A dilemma is encountered when choosing the resolution of input data to model rough surface contacts. On one hand, to accurately model the real rough surface contact, the resolution of the surface input should be high enough. It has long been known that material surfaces consist of asperities that are as small as nanoscale ( $10 \mathrm{~nm})$. Under certain circumstances, 
the local real contact areas are extremely small if single asperity contacts are formed. In such cases, high resolution of the surface input should be applied so that these small asperities can be depicted accurately. However, very high resolution of input is not always feasible due to the computational costs of SAM algorithms. For a simple elastic contact calculation, the complexity of the SAM algorithm is $\mathrm{O}\left(\mathrm{N}(\operatorname{logN})^{2}\right)$ and the memory consumption is $O\left(N^{2}\right)[1]$, suggesting that doubling the input resolutions in both $\mathrm{x}$ and $\mathrm{y}$ directions means eight calculations of time and four of memory demands. For some elasto-plastic contact cases, the complexity will be dramatically higher because the calculation domains are 3D instead of 2D for the simple elastic contact cases. Wang et al. reported an $864 \mathrm{MNL}^{*} \ln (8 \mathrm{MNL})+432 \mathrm{MNL}^{*} \ln (4 \mathrm{MN})$ complexity for an algorithm solving an arbitrarily shaped inclusion in an elasto-plastic contact [2]. Therefore, compromises on input resolutions sometimes have to be made in order that calculations can be completed within a reasonable time duration.

Due to this conflict, the effects of input surface resolution on the accuracy of SAM results need to be examined carefully. In this chapter, a study is made of the sensitivity on the maximum contact pressure and real contact area results with respect to the resolution of surface input. First, the contact between an analytically generated wavy surface and a rigid flat are studied and compared with the analytical solution. The investigation is then extended to a real surface contact, by studying the contacts of a steel ball and a grinded flat before and after running in.

\subsection{Real contact area between a wavy surface and a flat}

In this part, the sensitivity of real contact area results to the resolution of surface topography input is studied under a well-understood situation: the contact between an elastic wavy surface and a rigid flat. The real contact area is calculated both analytically and by the SAM model with different resolutions of input. The results are compared and the errors of the SAM results as a function of the resolution of input are investigated and discussed.

See Fig. 4.1, an elastic sinusoidal waviness is considered. The geometric shape of the sinusoidal wave is defined by Eq. (4-1), which is also illustrated in literature [3].

$$
z(x, y)=\Delta_{1} \cos \left(\frac{2 \pi x}{\lambda_{1}}\right)+\Delta_{2} \cos \left(\frac{2 \pi y}{\lambda_{2}}\right)
$$


where

$$
\Delta_{1}=\Delta_{2}=0.5 \mathrm{um}, \quad \lambda_{1}=\lambda_{2}=12.5 \mathrm{um}
$$

The size of the wavy surface is $250 \mu \mathrm{m} * 250 \mu \mathrm{m}$, while there are 20 waves in both $\mathrm{x}$ and y direction.

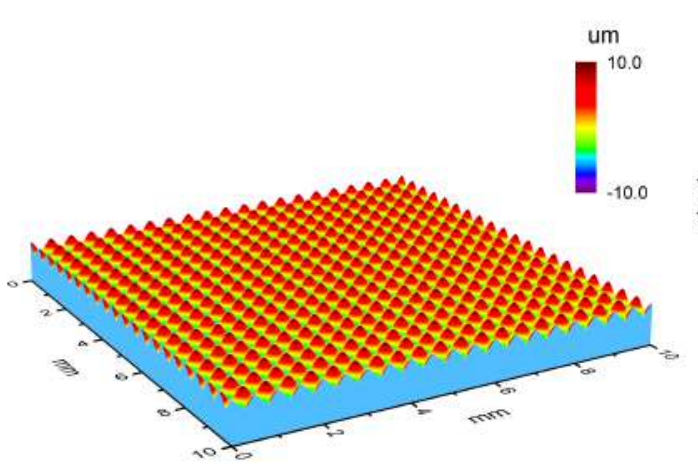

(a)

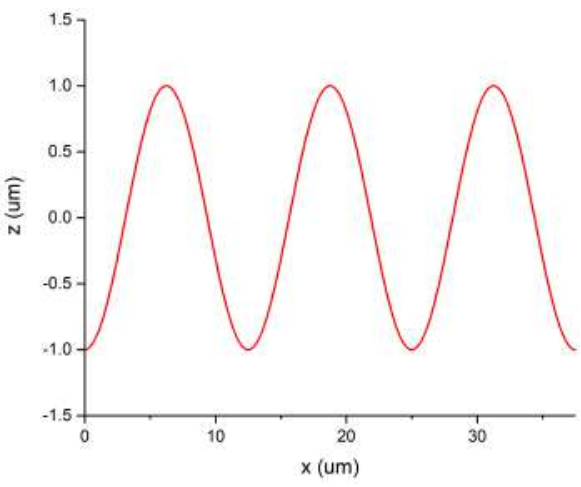

(b)

Fig. 4.1. The sinusoidal wavy surface: (a) 3D view and (b) cross section view.

The elastic modulus of this wavy surface is set to $210 \mathrm{GPa}$, the same as steel. Elastic contact between this surface and a rigid flat is considered. Three nominal contact pressures $(\overline{\boldsymbol{p}})$ being used are $50 \mathrm{MPa}, 100 \mathrm{MPa}$ and $200 \mathrm{MPa}$ respectively.

The analytical solutions of maximum contact pressure and real contact area are given in literature [3]:

$$
p_{a x}=\frac{3 \bar{p}}{2 \pi\left(\frac{3}{8 \pi} \frac{\bar{p}}{p^{*}}\right)^{\frac{2}{3}}}
$$

and 


$$
\frac{A_{\text {real }}}{A_{\text {nominal }}}=\pi\left(\frac{3}{8 \pi} \frac{\bar{p}}{p^{*}}\right)^{\frac{2}{3}}
$$

while

$$
p^{*}=\frac{2 \pi E^{*} \Delta}{\lambda}
$$

and

$$
\frac{1}{E^{*}}=\frac{1}{E_{1}}+\frac{1}{E_{2}}
$$

For the SAM modelling, the wavy surface is meshed into six different resolutions: $64 * 64,128 * 128,256 * 256,512 * 512,1024 * 1024$ and $2048 * 2048$ as inputs. The grid sizes of these inputs are $3.91 \mu \mathrm{m}, 1.95 \mu \mathrm{m}, 0.98 \mu \mathrm{m}, 0.49 \mu \mathrm{m}, 0.24 \mu \mathrm{m}$ and $0.12 \mu \mathrm{m}$ respectively. Then the contacts are solved by the method described in Appendix I. The results of the real contact area are presented in Tab. 4.1 and Fig. 4.2. 
Tab. 4.1. Analytical and SAM results of the real over nominal contact area ratio under different nominal loads

\begin{tabular}{|c|c|c|c|c|}
\hline \multirow{2}{*}{$\begin{array}{c}\text { Nominal } \\
\text { pressure (MPa) }\end{array}$} & \multirow{2}{*}{$\begin{array}{c}\text { Analytical } \\
\text { solution }\left(\mathrm{um}^{2}\right)\end{array}$} & \multicolumn{2}{|c|}{ SAM solution } & \multirow{2}{*}{ Error (\%) } \\
\hline & & Grid size $(\mu \mathrm{m})$ & Result $\left(\mu \mathrm{m}^{2}\right)$ & \\
\hline \multirow{6}{*}{50} & \multirow{6}{*}{445.6} & 3.91 & 1708 & 283.7 \\
\hline & & 1.95 & 1083 & 143.2 \\
\hline & & 0.98 & 763.1 & 71.3 \\
\hline & & 0.49 & 542.5 & 21.8 \\
\hline & & 0.24 & 491.9 & 8.5 \\
\hline & & 0.12 & 468.8 & 5.3 \\
\hline \multirow{6}{*}{100} & \multirow{6}{*}{706.9} & 3.91 & 2381 & 236.6 \\
\hline & & 1.95 & 1526 & 115.8 \\
\hline & & 0.98 & 1049 & 48.4 \\
\hline & & 0.49 & 828.8 & 17.2 \\
\hline & & 0.24 & 767.5 & 7.9 \\
\hline & & 0.12 & 740 & 4.6 \\
\hline \multirow{6}{*}{200} & \multirow{6}{*}{1123} & 3.91 & 3418 & 204.5 \\
\hline & & 1.95 & 2014 & 79.4 \\
\hline & & 0.98 & 1431 & 27.4 \\
\hline & & 0.49 & 1284 & 14.5 \\
\hline & & 0.24 & 1208 & 7.5 \\
\hline & & 0.12 & 1172 & 4.4 \\
\hline
\end{tabular}




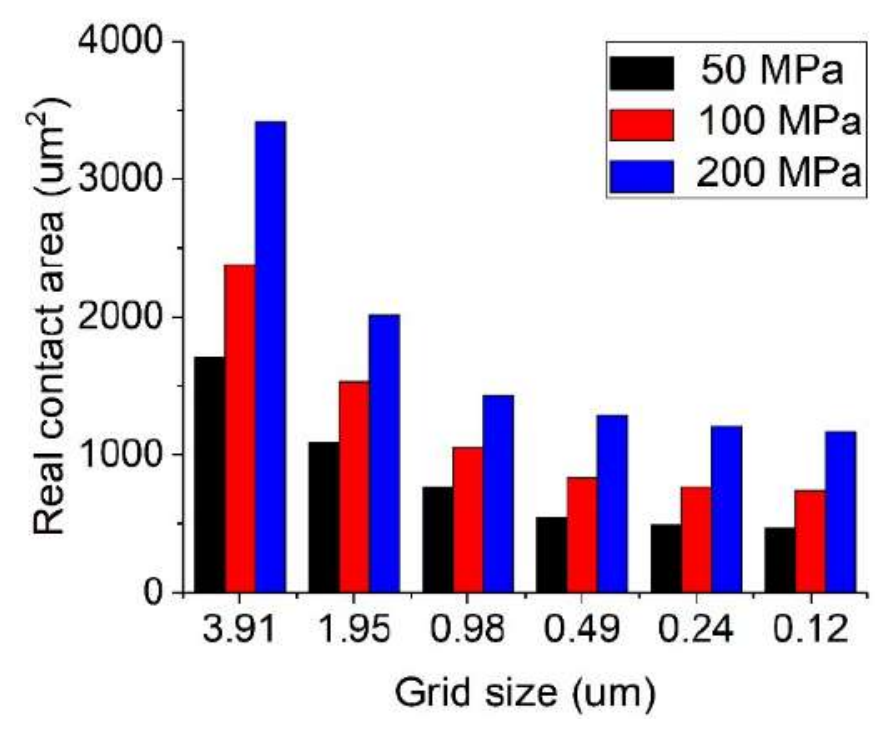

Fig. 4.2. Real contact area of the sinusoidal surface that contact calculated by the SAM.

Clearly, the accuracy of the SAM results is sensitive to the grid size: the smaller the grid size is, the smaller the relative error will be. The real contact area converges to the analytical solution when the grid size drops. However, it is worth noting that the errors of the SAM results cannot be negligible. Take the $100 \mathrm{MPa}$ case for example, when the grid size is $3.91 \mu \mathrm{m}$, the accuracy of the real contact area result is rather poor $(48.4 \%$ relative error). Even with the smallest grid size $(0.12 \mu \mathrm{m})$, the relative error of the real contact area still remains $4.61 \%$.

\subsection{Extrapolation of the SAM results}

The reason of the sensitivity of SAM results on grid size is that the calculation of real contact area is indirect: the algorithm first determines the contact pressure on each grid, and the grids with non-zero contact pressures will be marked as "in contact". Then the contact area will be calculated by the total area of the grids in contact. However, using a rectangle grid size, the grids will usually overly cover the contact boundaries. This "over-covering" effect leads to larger errors in calculating the real contact area. 
An elaborated demonstration is presented in Fig. 4.3. A close look of the calculated results of local contact on a single summit of the wavy surface are presented, under $100 \mathrm{MPa}$ nominal contact pressure. The resolutions of input being used are $256^{*} 256$, $512 * 512,1024 * 1024$, and $2048 * 2048$ respectively. It appears that with low input resolution $(256 * 256)$ while the grid sizes are large, the over-covering effect is quite severe (Fig. 4.3a). A rectangle shape (in this case a square) is considered by SAM as the real contact in this spot, while the actual contact area shape is a circle. Consequently, a large error occurs here. With higher input resolutions, the sizes of grids become smaller, the over-covering effect become less severe, and more accurate coverages are achieved (Fig. 4.3b, 4.3c and 4.3d). This provides a higher accuracy of the numerical results. Note that this over-covering effect exists only in calculating the real contact area, while the calculation of the contact pressures is hardly affected by this. Therefore, the SAM shows much higher sensitivity to input grid size when calculating the real contact area then the contact pressure.

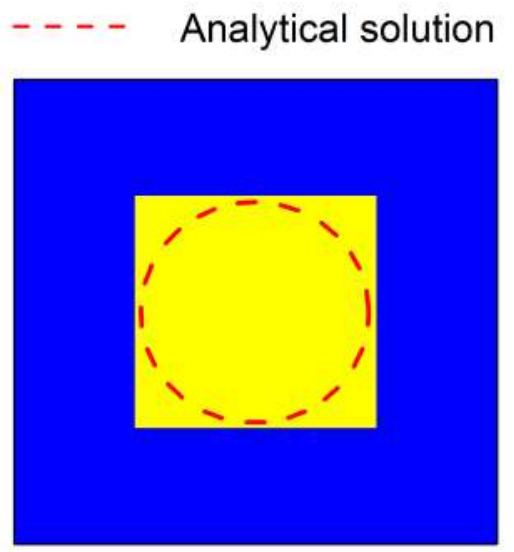

(a)

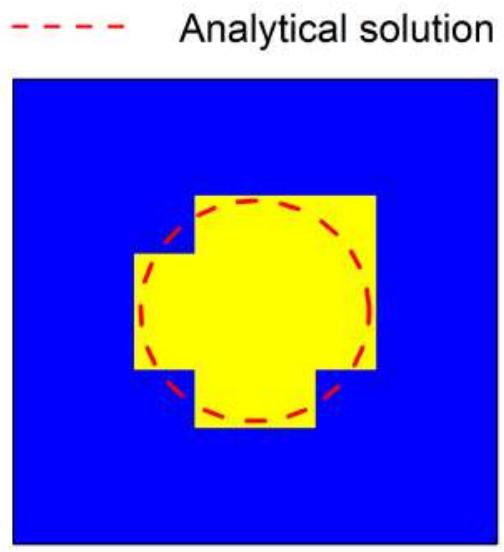

(b) 


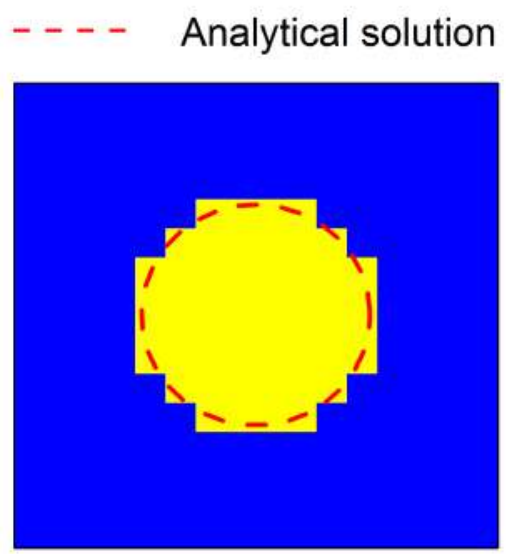

(c)
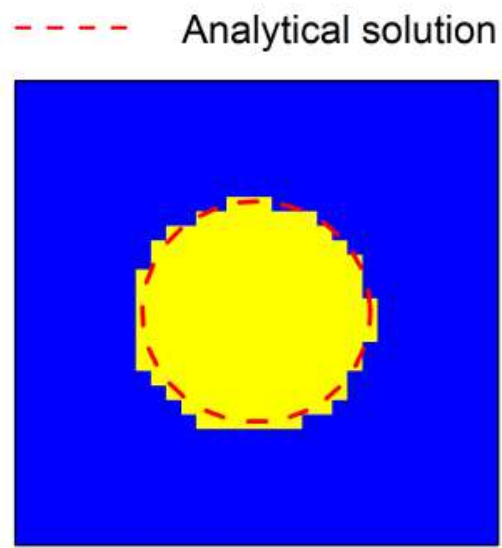

(d)

Fig. 4.3. SAM results with different grid sizes (yellow grids are in contact and blue grids are not in contact): (a) $0.98 \mu \mathrm{m}$; (b) $0.49 \mu \mathrm{m}$; (c) $0.24 \mu \mathrm{m}$ and (d) $0.12 \mu \mathrm{m}$.

Here, an extrapolation method is proposed to acquire more accurately the contact areas when further improving the input resolution is impossible or inconvenient. $A$ three-parameter decayed exponential formula is used to fit the data points:

$$
A=k e^{-\frac{x}{t}}+A_{0}
$$

In this formula, $\boldsymbol{A}$ is the real contact area calculated by SAM, $\mathrm{x}$ is the number of grids in $x$ or $y$ direction, and $\boldsymbol{k}, \boldsymbol{t}$ and $\boldsymbol{A}_{\mathbf{0}}$ are the three fitting parameters. The term $\boldsymbol{k} \boldsymbol{e}^{-\frac{\boldsymbol{x}}{\boldsymbol{t}}}$ can be regarded as the error term caused by the over-covering effect. When the resolution term $x$ goes larger, the value of this term will decrease, and eventually converge to zero when the grid size goes to infinite. Then the term $\boldsymbol{A}_{\mathbf{0}}$ will be the predicted real contact area.

This technique is applied to the real contact area results of the wavy surface, see Fig. 4.4. The exponential functions achieve satisfactory fitting accuracies for all three contact cases. For each data set, the six data points fall fairly close to the fitting curve. 
In addition, the fitting curves converge accurately to the analytical solution. See Tab.

4.2.

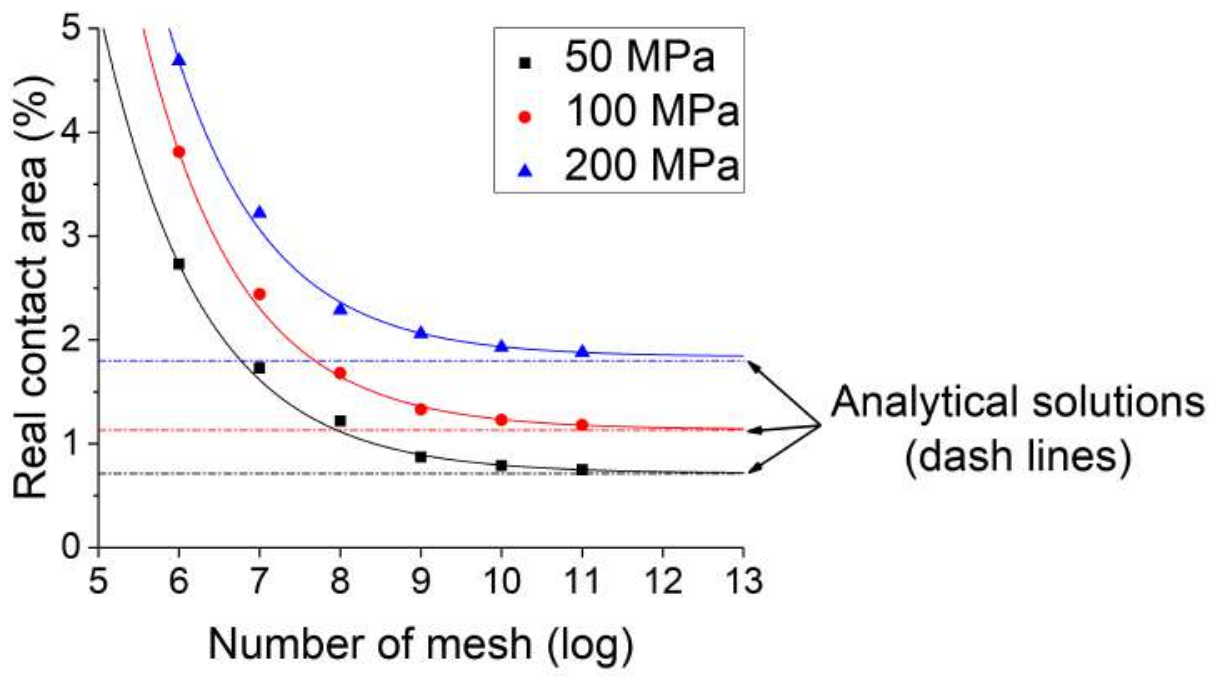

Fig. 4.4. Exponential extrapolation of real contact area of the wavy surface against a flat.

Tab. 4.2. Comparison of extrapolation results and analytical results.

\begin{tabular}{cccc}
\hline $\begin{array}{c}\text { Nominal pressure } \\
(\mathrm{MPa})\end{array}$ & $\begin{array}{c}\text { Analytical solution } \\
\left(\mu \mathrm{m}^{2}\right)\end{array}$ & $\begin{array}{c}\text { Extrapolation results } \\
\left(\mu \mathrm{m}^{2}\right)\end{array}$ & $\begin{array}{c}\text { Error } \\
(\%)\end{array}$ \\
\hline 50 & 445.6 & 446.3 & 0.14 \\
100 & 706.9 & 710.6 & 0.45 \\
200 & 1123 & 1149 & 2.33 \\
\hline
\end{tabular}

Based on the foregoing discussion, it can be seen that exponential extrapolation can be employed to predict the real contact area value based on a series of results from different grid sizes. This technique can be further applied to the real rough surface contact calculation, which is presented in Section 4.4. 


\subsection{Real rough surface contact}

In this part, the elasto-plastic contact between the pin-on-disc samples (see Fig. 4.5) is studied to investigate the sensitivity of the SAM results with respect to the resolutions of input. Both the ball and the ring are made of 52100 bearing steel. The mechanical properties are shown in Tab. 4.3.

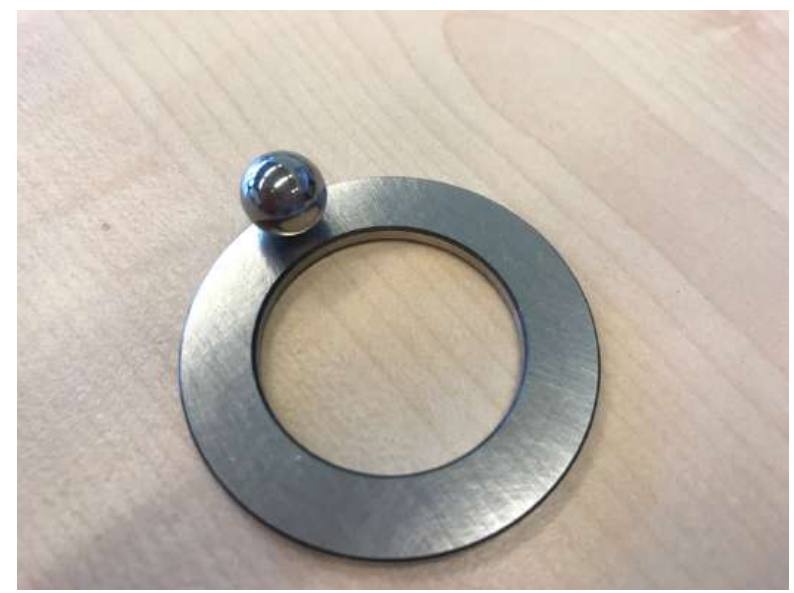

Fig. 4.5. Ball and flat surfaces samples.

Tab. 4.3. Chemical composition of AISI 52100.

\begin{tabular}{cc}
\hline Element & Content (\%) \\
\hline $\mathrm{C}$ & $0.980-1.10$ \\
$\mathrm{Cr}$ & $1.30-1.60$ \\
$\mathrm{Fe}$ & $96.5-97.32$ \\
$\mathrm{Mn}$ & $0.250-0.450$ \\
$\mathrm{P}$ & $\leq 0.0250$ \\
$\mathrm{~S}$ & $\leq 0.0250$ \\
$\mathrm{Si}$ & $0.150-0.300$ \\
\hline
\end{tabular}

Two surface conditions are considered: before and after running in. The running in process is carried out by a pin-on-disc testing machine. The surface data are acquired 
by a confocal microscope SENSOFAR S Neox. The measuring domains are originally 264.2um*264.2um, with 2048*2048 grid size, as presented in Fig. 4.6 and Fig. 4.7.

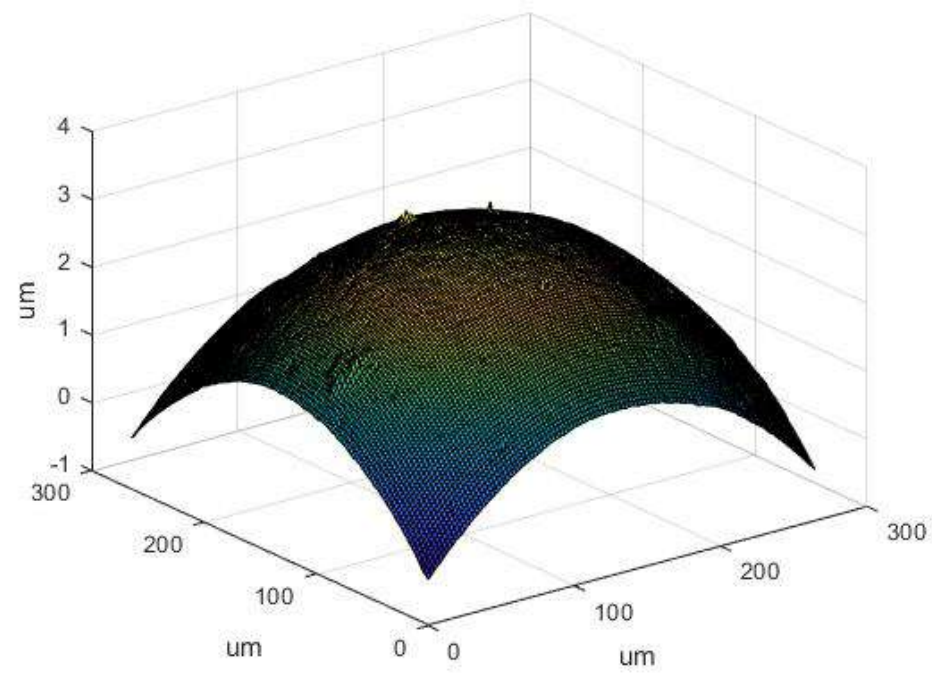

(a)

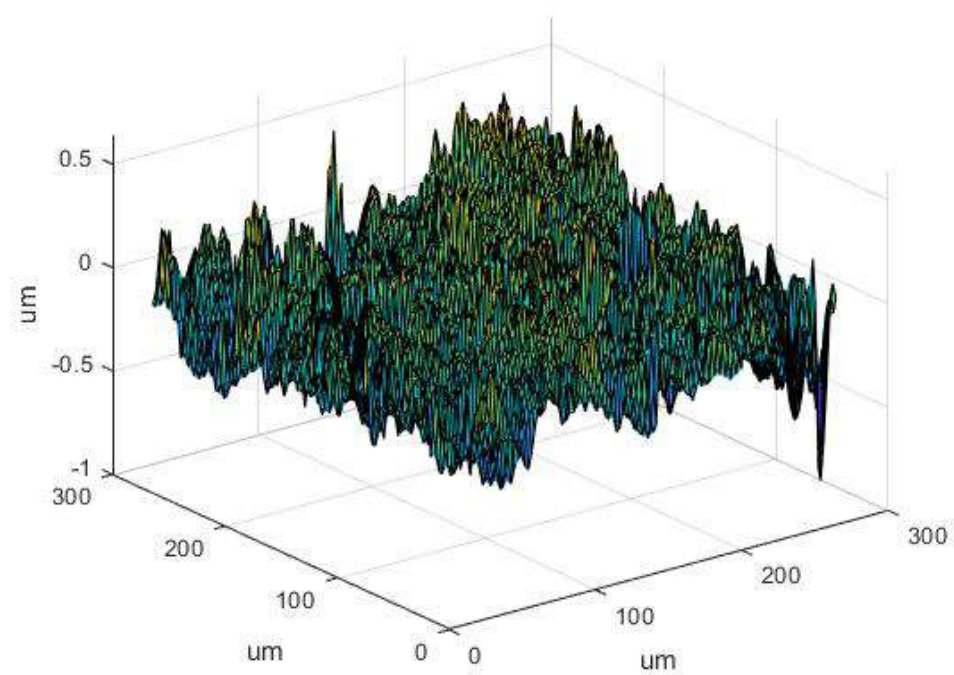

(b)

Fig. 4.6. Surface profiles of samples before running in: (a) ball and (b) ring. 


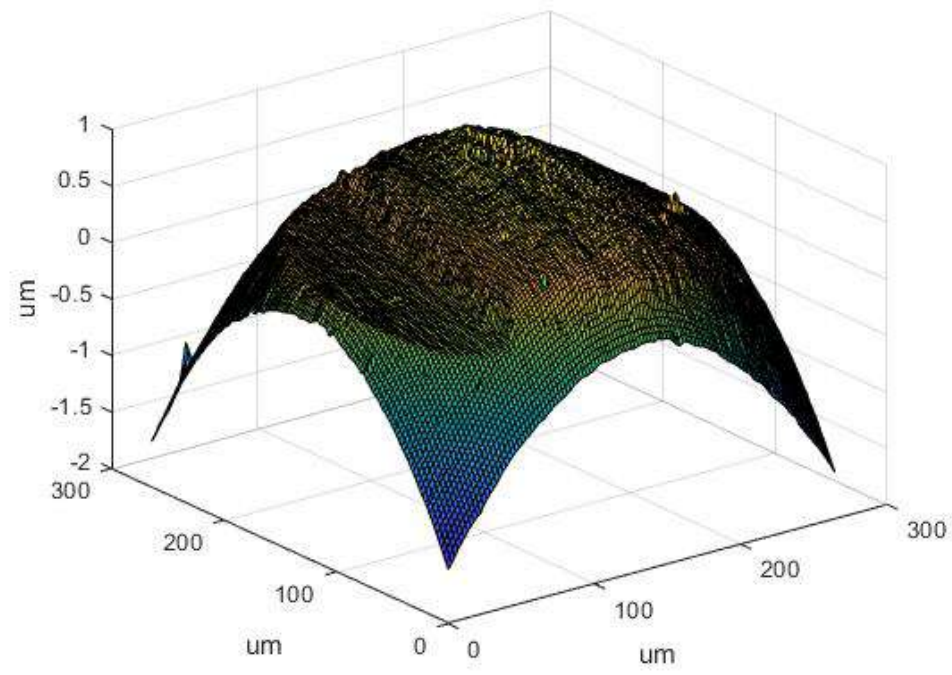

(a)

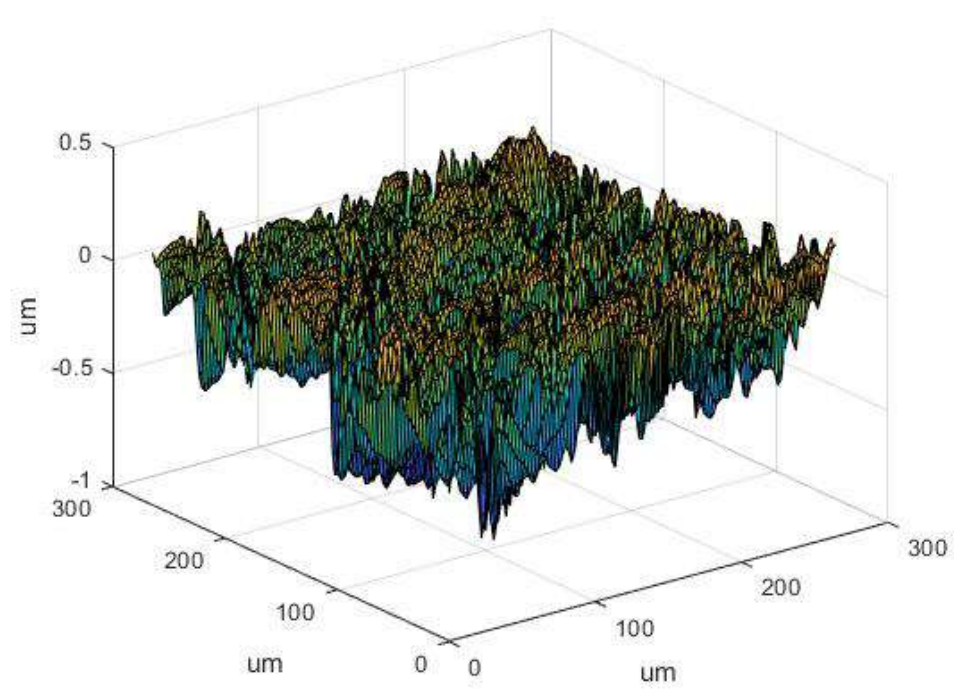

(b)

Fig. 4.7. Surface profiles of samples after running in: (a) ball and (b) ring.

In order to investigate the effect of resolution, the surface data are scaled into five different lower resolutions $64 * 64,128 * 128,256 * 256,512 * 512,1024 * 1024$ by the 
bilinear scaling algorithm. Together with the original 2048*2048 data, six different resolutions of input are acquired. Three different normal loads are applied, which are $2.5 \mathrm{~N}, 5 \mathrm{~N}$ and $10 \mathrm{~N}$ respectively. Considering the elasto-plastic property of the material, the maximum contact pressure is set to $6 \mathrm{GPa}$, the same as the hardness. A single elastic, perfectly plastic curve is employed in the calculation.

The calculated real contact areas with different surface input grid sizes are shown in Fig. 4.8. The results indicate that for the samples before running in the real contact areas converge relatively well, while for the samples after running in the results show substantial differences even with high input resolutions. Therefore, the accuracies of the calculated real contact area values remain questionable.

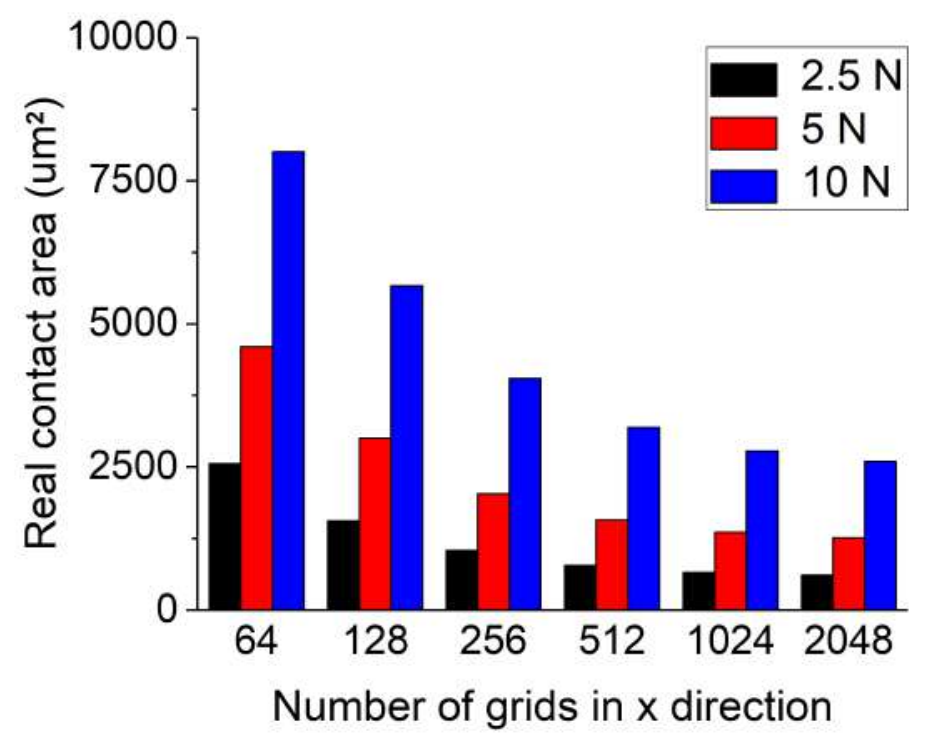

(a) 


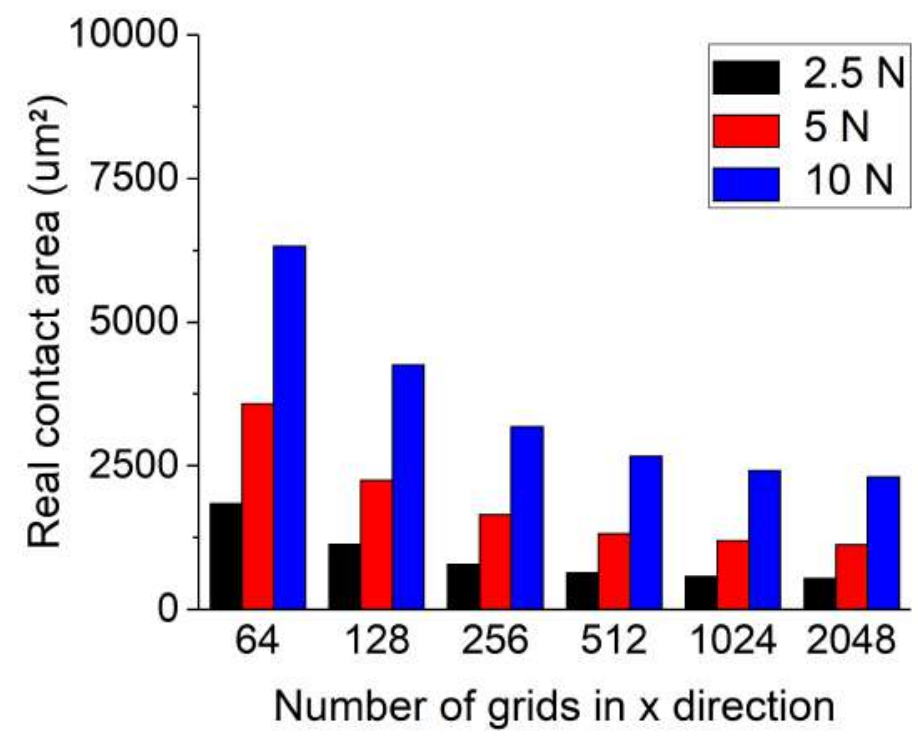

(b)

Fig. 4.8. Real contact areas calculated by SAM with different resolutions of input:

(a) before running in and (b) after running in.

The real contact areas are predicted by the fitting technique proposed in Subsection 3.1 and shown in Fig. 4.9. The results show that for both cases, the results from the different input resolutions fall on the exponential curves nicely. The predicted results are compared with the SAM results with the highest resolution $(2048 * 2048)$ in Tab. 4.4. 


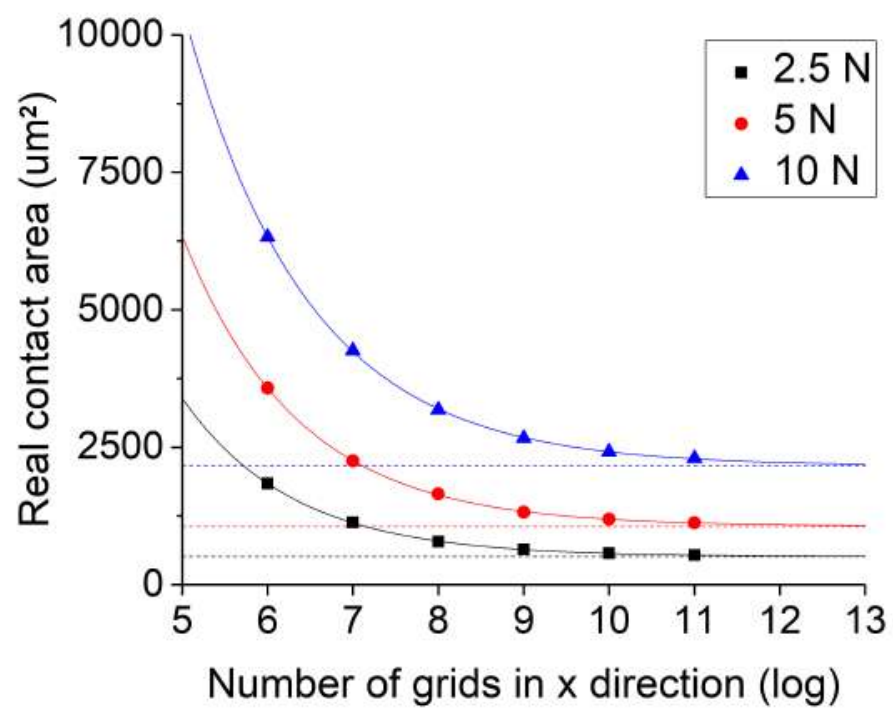

(a)

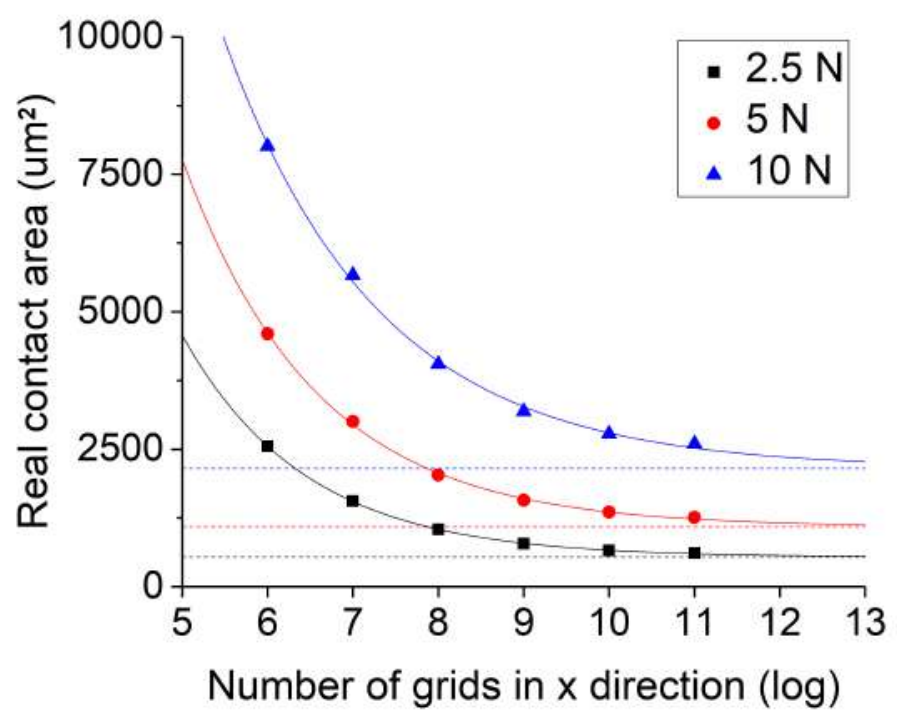

(b)

Fig. 4.9. Exponential extrapolation of real contact areas of the samples: (a) before running in and (b) after running in. 
Tab. 4.4. Comparison of real contact area: SAM results and extrpolation results of samples.

\begin{tabular}{ccccc}
\hline Samples & Normal load $(\mathrm{N})$ & $\begin{array}{c}\text { SAM results } \\
(2048 * 2048) \\
\left(\mu \mathrm{m}^{2}\right)\end{array}$ & $\begin{array}{c}\text { Extrapolation results } \\
\left(\mu \mathrm{m}^{2}\right)\end{array}$ & $\begin{array}{c}\text { Improvement } \\
(\%)\end{array}$ \\
\hline \multirow{2}{*}{ Before running in } & 2.5 & 540.1 & 509.9 & 5.9 \\
& 5 & 1126.2 & 1063.1 & 5.9 \\
\hline \multirow{2}{*}{ After running in } & 10 & 2301.2 & 2159.6 & 6.6 \\
\hline & 5 & 613.9 & 538.3 & 14.1 \\
& 10 & 1262.4 & 1086.9 & 16.2 \\
\hline
\end{tabular}

\subsection{Summary}

In this chapter, the sensitivity of the SAM contact model on the input grid size is examined. An extrapolation method is proposed to predict the real contact area when further reducing the grid size is inconvenient. The primary conclusions are summarized below.

(1) The results of the real contact area calculated by the SAM model is sensitive to the input grid size. This is due to the indirect way that the SAM model calculates the real contact area. Over-covering effects occur on the edge of the real contact areas and cause overestimation of the real contact areas.

(2) In real rough surface contacts, the over-covering effect of SAM can be severe, especially in the cases where the contact pressures are dispersely distributed. In these cases, local contact areas are quite small: similar or even smaller than the size of the grids. As a consequence, the calculated real contact area may significantly deviate from the real values.

(3) The exponential extrapolation can be employed to predict the real contact area when further improvement the grid size of input is difficult.

\section{References}


[1] Polonsky, I. A., \& Keer, L. M. (1999). A numerical method for solving rough contact problems based on the multi-level multi-summation and conjugate gradient techniques. Wear, 231(2), 206-219.

[2] Wang, Z., Jin, X., Zhou, Q., Ai, X., Keer, L. M., \& Wang, Q. (2013). An efficient numerical method with a parallel computational strategy for solving arbitrarily shaped inclusions in elastoplastic contact problems. Journal of Tribology, 135(3), 031401.

[3] Johnson, K. L., Greenwood, J. A., \& Higginson, J. G. (1985). The contact of elastic regular wavy surfaces. International Journal of Mechanical Sciences, 27(6), 383-396. 



\section{A combined friction model for boundary lubrication}

In this chapter, the contributions of the deformation force and adhesion force to the total friction level of the pin-on-disc tribosystem are studied separately. The total friction levels are calculated and compared with the results measured by the experiments.

\subsection{Introduction}

This chapter combines the findings of the previous chapters and the friction contributed by the asperity deformation force and the surface adhesion force are calculated. The deformation force is calculated by applying the single asperity model developed in Chapter 3. The surface adhesion force is calculated by evaluating the real contact area using the method proposed in Chapter $\mathbf{4}$, and the forces are calculated with the shear strength reported in Chapter $\mathbf{2}$.

\subsection{Calculation of the deformation force}

In this part, the friction caused by asperity deformation of the tribosystem is calculated by the single asperity sliding model developed in Section 3.2. First, the modelling input (asperity radius and interference depth) is determined, then the results are summarized and discussed.

\subsubsection{Modelling parameters}

(1) Asperity recognition and average radius

An asperity is defined as a point with a local surface height greater than its neighbouring points. Different definitions including five-point summits and ninepoint summits can be used to characterize the surface asperities. De Rooij [1] advised using the nine-point definition because this minimizes the probability of detecting "false summits". This comes down to: 


$$
z_{x, y}>z_{x-1, y-1}, z_{x-1, y}, z_{x-1, y+1}, z_{x, y-1}, z_{x, y+1}, z_{x+1, y-1}, z_{x+1, y}, z_{x, y+1}
$$

With the summit points decided, the summit radius $\boldsymbol{\beta}_{\boldsymbol{i}}$ can be calculated based on a quadratic interpolation:

$$
\begin{aligned}
& \beta_{x, i}=\frac{r_{x}^{2}}{z_{x-1, y}-2 z_{x, y}+z_{x+1, y}} \\
& \beta_{y, i}=\frac{r_{y}^{2}}{z_{x, y-1}-2 z_{x, y}+z_{x, y+1}}
\end{aligned}
$$

where $\boldsymbol{z}_{\boldsymbol{x}, \boldsymbol{y}}$ is the local surface height at summit location $(\mathrm{x}, \mathrm{y})$ and $\boldsymbol{r}_{\boldsymbol{x}}, \boldsymbol{r}_{\boldsymbol{y}}$ denote the interferometer pixel size in $\mathrm{x}$ and $\mathrm{y}$ direction respectively.

Surface profile samples of the asperities on the ball and the disc surfaces are presented in Fig. 5.1. The asperities on the ball surfaces are more evenly distributed with similar shapes, while the asperities on the disc surfaces vary in size and distribution.

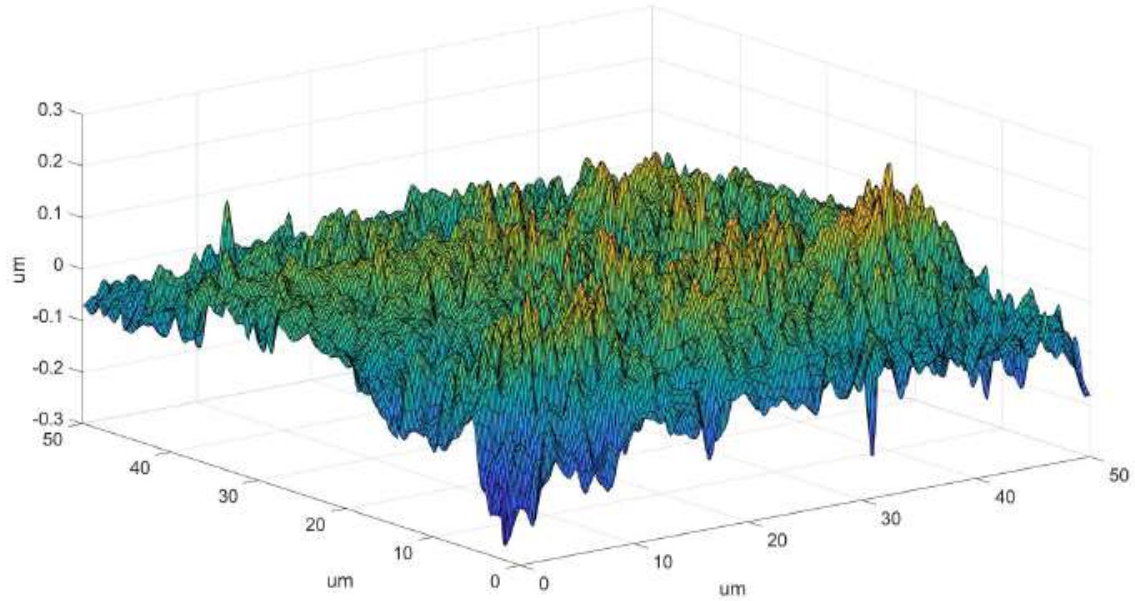

(a) 


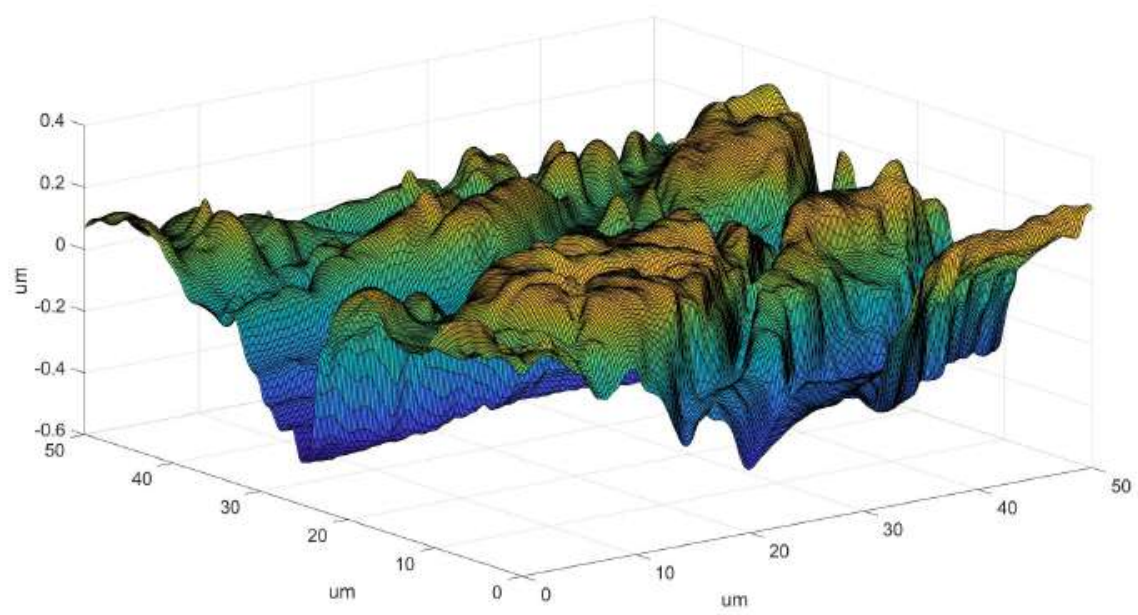

(b)

Fig. 5.1. Surface profiles of pin-on-disc samples (tested at $150^{\circ} \mathrm{C}$ ): (a) ball and (b) disc.

The average asperity radii of the ball and disc surfaces tested under different temperatures are given in Tab. 5.1. It is evident that despite the friction and wear levels are different, the asperity sizes (average radii) are comparable. This is in line with the findings in Section $\mathbf{2 . 3}$ that the roughness of the samples tested under different temperatures are similar.

Tab. 5.1. Average asperity radii of ball and disc surfaces.

\begin{tabular}{ccc}
\hline \multirow{2}{*}{ Temperature $\left({ }^{\circ} \mathrm{C}\right)$} & Sample & Avg. radius $(\mu \mathrm{m})$ \\
\hline \multirow{2}{*}{80} & Disc & 6.1 \\
& Ball & 15.7 \\
\hline \multirow{2}{*}{150} & Disc & 6.6 \\
& Ball & 18.1 \\
\hline
\end{tabular}

(2) Average interference depth 
The surface deformation of the ball under different normal loads are shown in Fig. 5.2 and Fig. 5.3. It can be clearly seen that the total surface deformation consists of two parts: (1) macro-deformation which occurs globally, caused by the macro-shape of ball and flat, and (2) local micro-deformations that occur solely at the real contact areas, caused by the asperity contact.

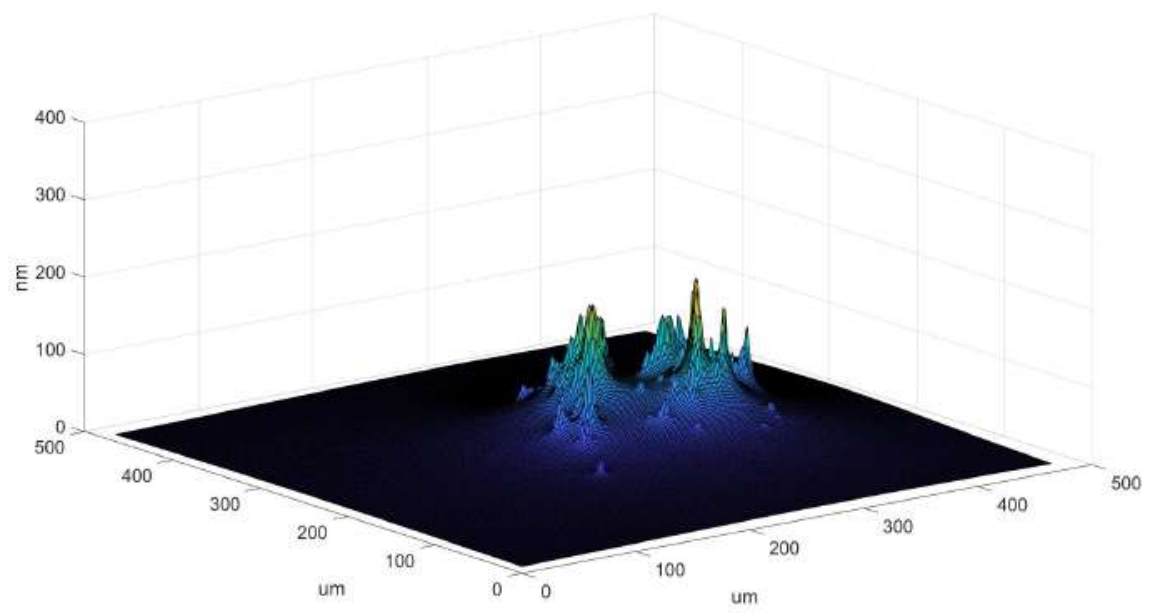

(a)

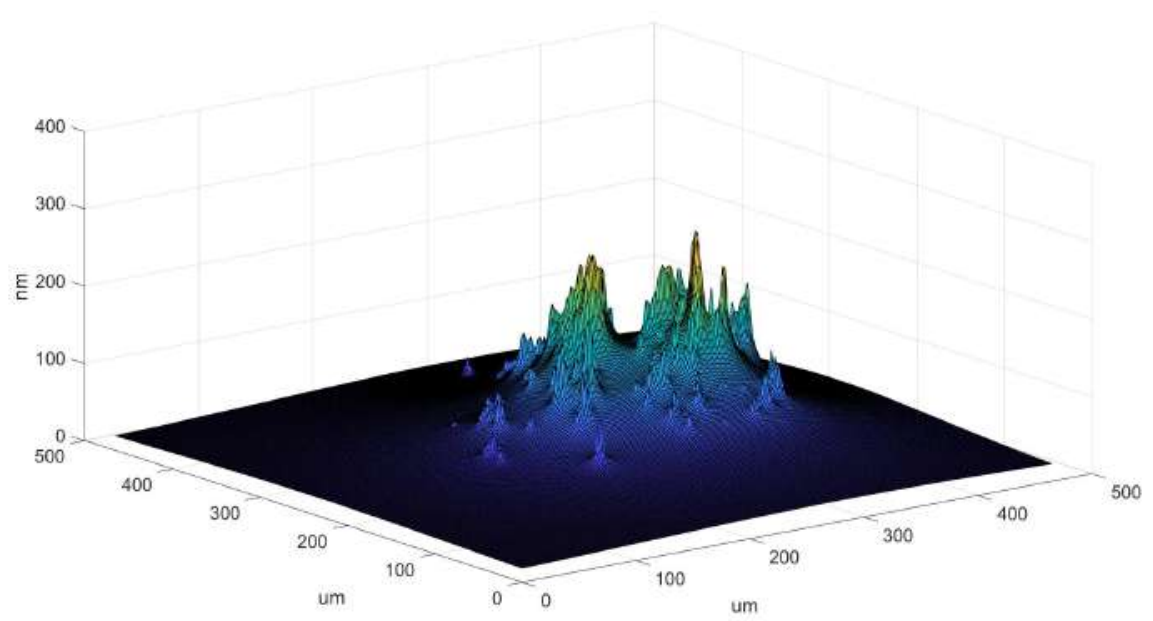

(b)

Fig. 5.2. Surface deformation of samples tested under different loads (both tested at $150^{\circ} \mathrm{C}$ ): (a) $2 \mathrm{~N}$ and (b) $5 \mathrm{~N}$. 


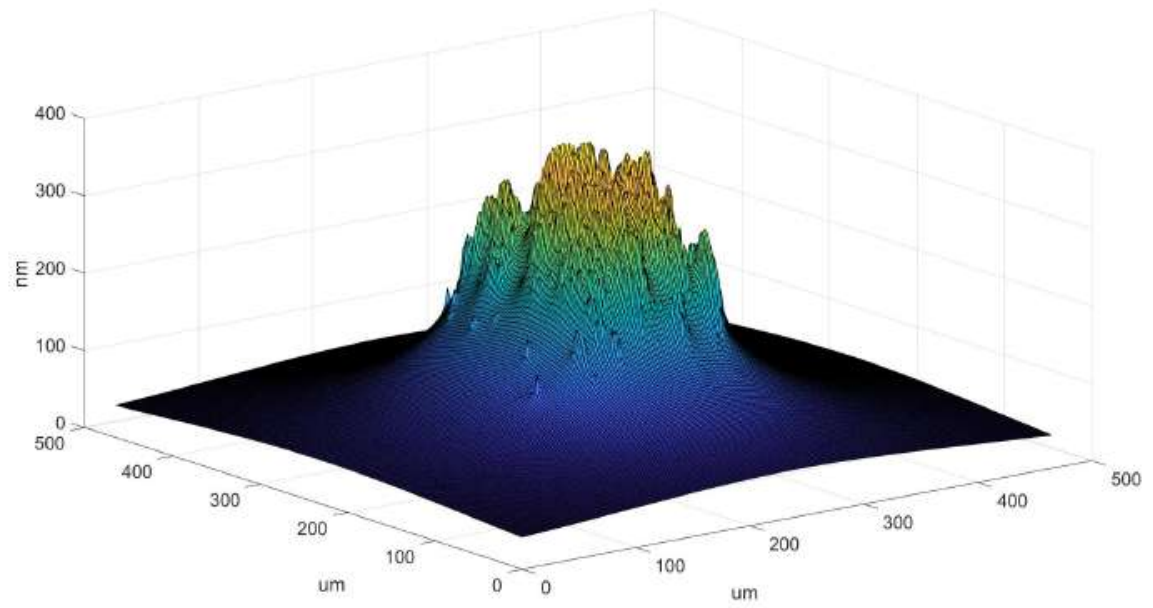

(a)

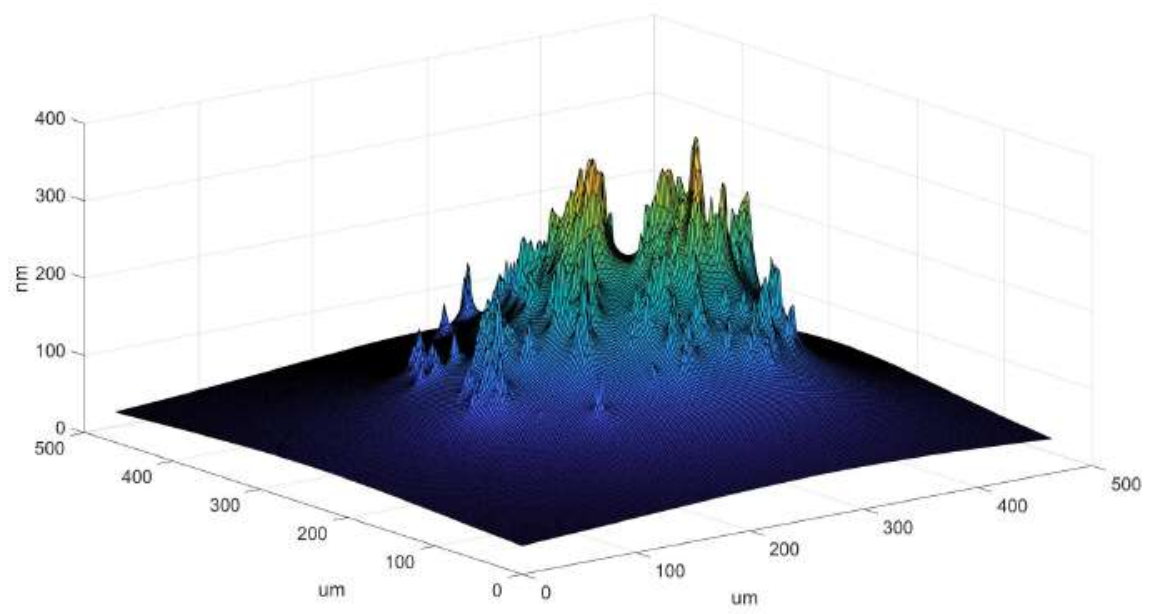

(b)

Fig. 5.3. Surface deformation of samples tested at different temperatures (both under $10 \mathrm{~N}$ normal load): (a) $80^{\circ} \mathrm{C}$ and (b) $150^{\circ} \mathrm{C}$. 
It can be found from Fig. $\mathbf{5 . 2}$ and Fig. $\mathbf{5 . 3}$ that normal load affects the macrodeformation more than the micro-deformation. This is due to the elasto-plastic nature of the surface. The macro-deformations are determined mainly by the elastic properties of the surface, indicating a linear feedback of the normal loads. The microdeformation, however, is strongly affected by the plastic flow, due to the very high local pressure. In this case, local asperities will be plastically deformed and will alter the surface shape, and the local micro-deformation will be reduced. This means the micro-deformations will not grow linearly with the normal load.

For the single asperity sliding, the interference depth is only related to the local micro-deformation. Note that because the two contact surfaces undergo similar deformations, the interference depth value should be taken as twice that of the micro-deformation value of either surface. The calculated results are listed in Tab.

5.2.

Tab. 5.2. Average interference depths of asperities.

\begin{tabular}{ccc}
\hline Temperature $\left({ }^{\circ} \mathrm{C}\right)$ & Normal load $(\mathrm{N})$ & Avg. depth $(\mathrm{nm})$ \\
\hline \multirow{3}{*}{80} & 2 & 101 \\
& 5 & 120 \\
& 10 & 144 \\
\hline \multirow{3}{*}{150} & 2 & 160 \\
& 5 & 187 \\
& 10 & 211 \\
\hline
\end{tabular}

\subsubsection{Friction forces of single asperity sliding}

With the radii of the asperities and the interference depths determined, and the mechanical properties of the tribofilm measured in Chapter 2, the sliding friction forces can be calculated by the single asperity sliding model proposed in Section 3.1.

Detailed coefficient of friction results are calculated and listed in Tab. 5.3. The results show that the asperity deformation of the samples tested at $150^{\circ} \mathrm{C}$ contributes more friction than the $80^{\circ} \mathrm{C}$ case due to the larger interference depths. However, for both cases, the contribution of the elasto-plastic deformation of asperities on the 
coefficient of friction due to deformation is fairly small, in the range of approximately 0.005 to 0.015 .

Tab. 5.3. Average coefficient of friction results.

\begin{tabular}{ccc}
\hline \multirow{2}{*}{ Temperature $\left({ }^{\circ} \mathrm{C}\right)$} & Normal load $(\mathrm{N})$ & COF \\
\hline \multirow{2}{*}{80} & 2 & 0.003 \\
& 5 & 0.004 \\
& 10 & 0.006 \\
\hline \multirow{2}{*}{150} & 2 & 0.007 \\
& 5 & 0.008 \\
& 10 & 0.013 \\
\hline
\end{tabular}

Therefore, it is concluded that the asperity deformation accounts for only a small fraction of the total deformation. The friction contributed by the shear of interfaces should be investigated.

\subsection{Calculation of the adhesion forces}

In this part, the friction caused by the adhesion force of the pin-on-disc tribosystem is considered. First, the real contact areas are calculated by the method proposed in Chapter 4. The adhesion forces are subsequently calculated by Eq. (1-3).

\subsubsection{Real contact areas of the tribosystem}

The real contact areas of the pin-on-disc samples are calculated by the SAM model. The surface profiles of the ball and five sites on the disc are measured by the confocal microscope and serve as the input of the SAM model. Example results are shown in Fig. 5.4 and Fig. 5.5 (calculated with 1024*1024 mesh). 


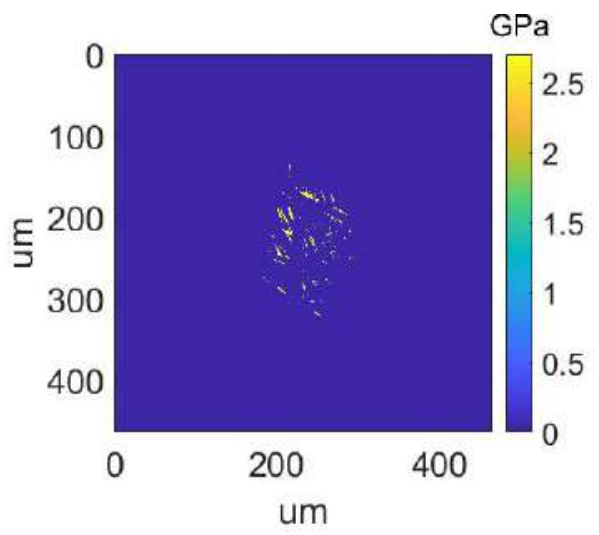

(a)

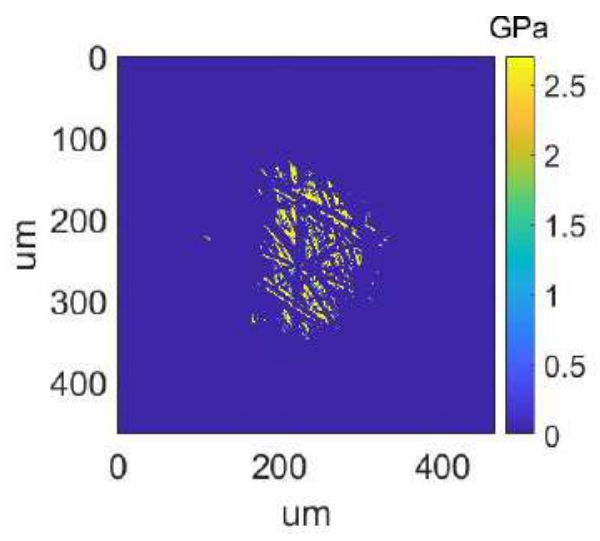

(c)

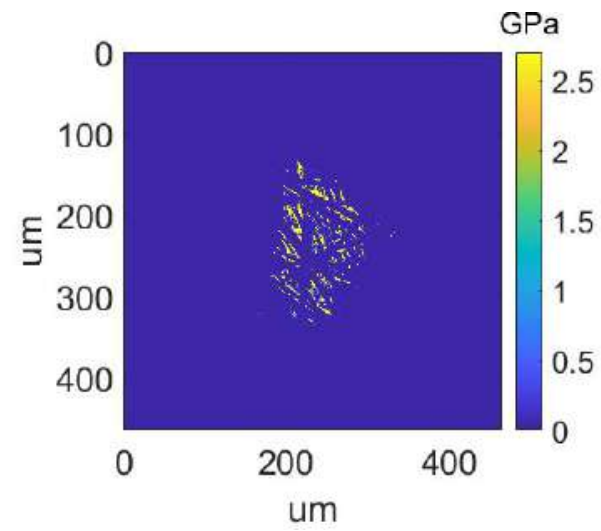

(b)

Fig. 5.4. Contact area and pressure distribution of samples tested at $80^{\circ} \mathrm{C}$ :

(a) $2 \mathrm{~N}$; (b) $5 \mathrm{~N}$ and (c) $10 \mathrm{~N}$. 


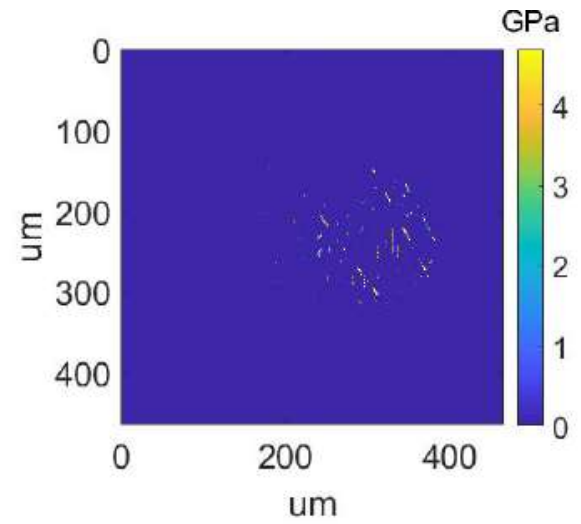

(a)

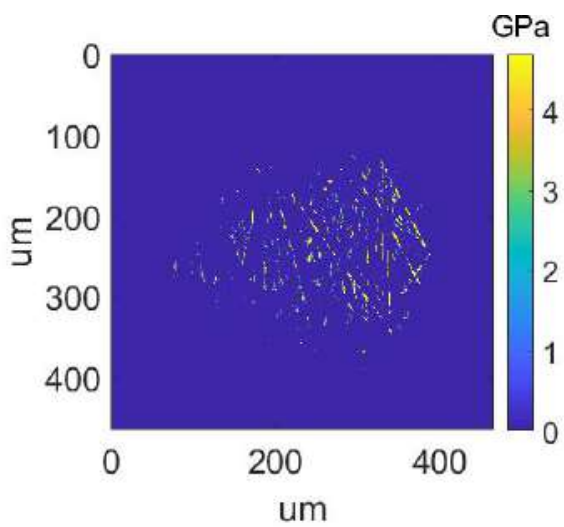

(c)

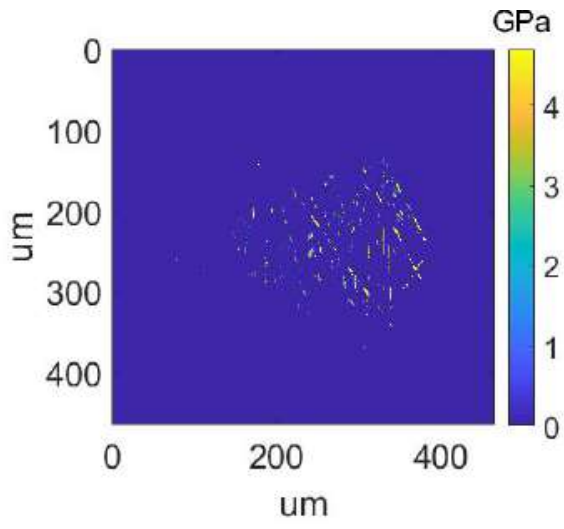

(b)

Fig. 5.5. Contact area and pressure distribution of samples tested at $150^{\circ} \mathrm{C}$ :

(a) $2 \mathrm{~N}$; (b) $5 \mathrm{~N}$ and (c) $10 \mathrm{~N}$.

The results show the contact pressures as well as the real contact areas. It can be noticed that for the $150^{\circ} \mathrm{C}$ case, the macro contact areas are greater than the $80^{\circ} \mathrm{C}$ cases. This is due to the fact that at $150{ }^{\circ} \mathrm{C}$, more wear occurs and as a result the wear scar sizes are larger. Meanwhile, the pressure distributions of the $150^{\circ} \mathrm{C}$ cases are more disperse. This is caused by the tribofilm properties. The hardness of the tribofilm at $150^{\circ} \mathrm{C}$ is $4.7 \mathrm{GPa}$, while at $80^{\circ} \mathrm{C}$ the hardness is $2.7 \mathrm{GPa}$. A softer tribofilm means more plastic flow. The surface shapes will be flattened more for the $80^{\circ} \mathrm{C}$ case, which leads to more continuous pressure distributions. 
Note that sensitivity of the grid size is observed. Therefore, the real contact areas are adjusted by the method proposed in Chapter 4. The results are presented in Tab.

5.4 .

Tab. 5.4. Average real contact areas under different test conditions.

\begin{tabular}{ccc}
\hline Temperature $\left({ }^{\circ} \mathrm{C}\right)$ & Normal load $(\mathrm{N})$ & $\begin{array}{c}\text { Avg. real contact area } \\
\left(\mathrm{um}^{2}\right)\end{array}$ \\
\hline \multirow{2}{*}{80} & 2 & $827 \pm 73$ \\
& 5 & $2183 \pm 250$ \\
150 & 10 & $4233 \pm 667$ \\
\hline \multirow{2}{*}{150} & 5 & $760 \pm 55$ \\
& 10 & $1995 \pm 175$ \\
& & $4010 \pm 550$
\end{tabular}

The coefficient of friction can be further calculated by Eq. (1-3). The results are listed in Tab. 5.5.

Tab. 5.5. Average coefficient of frictions under different test conditions.

\begin{tabular}{ccc}
\hline Temperature $\left({ }^{\circ} \mathrm{C}\right)$ & Normal load $(\mathrm{N})$ & Coefficient of friction \\
\hline \multirow{2}{*}{80} & 2 & $0.124 \pm 0.011$ \\
& 5 & $0.131 \pm 0.015$ \\
& 10 & $0.127 \pm 0.020$ \\
\hline \multirow{2}{*}{150} & 2 & $0.152 \pm 0.011$ \\
& 5 & $0.160 \pm 0.014$ \\
& 10 & $0.160 \pm 0.022$ \\
\hline
\end{tabular}


The modelling results are compared with the experiments in Fig. 5.6. For the $80^{\circ} \mathrm{C}$ case, the modelling results fit relatively well with the experiments; for the $150^{\circ} \mathrm{C}$ case, however, noticeable differences are found. This can be attributed to the fact that the tribofilm properties are all measured at room temperature. The shear strengths of the tribofilms under $150^{\circ} \mathrm{C}$ may be overestimated, which lead to higher calculated friction forces.

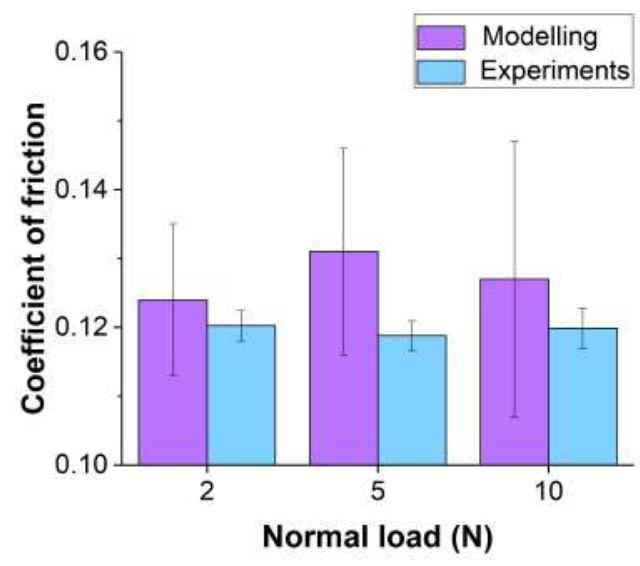

(a)

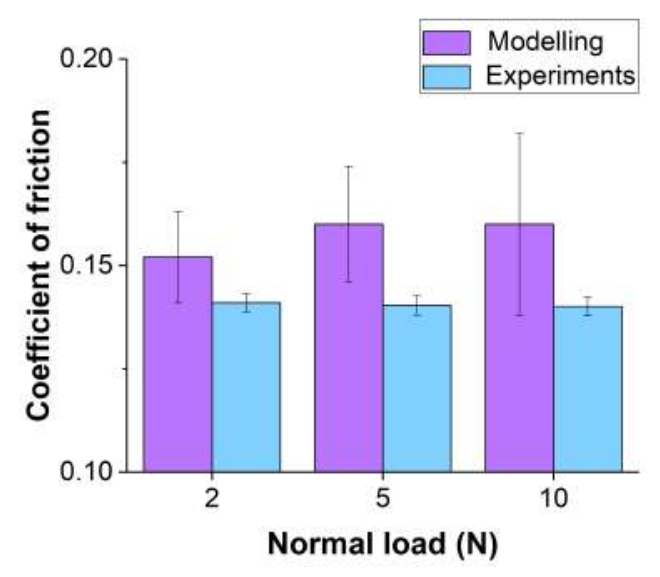

(b)

Fig. 5.6. Comparison of modelling and experimental results:

(a) $80^{\circ} \mathrm{C}$ and (b) $150^{\circ} \mathrm{C}$. 


\subsection{Summary}

In this chapter, the friction in a pin-on-disc tribosystem is investigated. The contributions of the asperity deformation forces and the adhesion forces are calculated separately based on the methods proposed in Chapter $\mathbf{3}$ and Chapter $\mathbf{4}$. The findings are summarized briefly below.

(1) The radii of the asperities of ball and disc samples are approximately at the 10 $\mu \mathrm{m}$ level, while the interference depths of asperities are around the 100-200 nm level. The results from the single asperity sliding model shows that with these given values, the deformation forces generated account for only a small fraction of the total friction level: the deformation forces result in a coefficient of friction at around the 0.005 to 0.015 level, which is only about 5 to $10 \%$ of the total measured coefficient of friction (0.12-0.14).

(2) The adhesion forces of the pin-on-disc samples tested at different temperatures are calculated. The results show that the real contact areas of samples tested at $150{ }^{\circ} \mathrm{C}$ are smaller and more dispersely distributed than the $80^{\circ} \mathrm{C}$ case, mainly due to the higher hardness of the tribofilm. The friction calculated by the model achieve fair agreement with the experimental data.

(3) It is proposed that in the current pin-on-disc tribosystem, the main mechanism of the sliding friction is the tribofilm adhesion. The shear strength of the tribofilm together with the real contact area is dominant with respect to the sliding friction level. The deformation of asperities do not play a significant role in this tribosystem with the given test conditions.

\section{References}

[1] de Rooij, M. B. (1998). Tribological aspects of unlubricated deep drawing processes. FEBO Druck. 


\section{Conclusions and recommendations}

The overall conclusions are summarized briefly in this chapter. Subsequently, the recommendations for future research are proposed.

\subsection{Conclusions}

\subsubsection{Effect of testing temperatures on the tribofilm properties}

The experimental work on tribofilm properties is presented in Chapter 2 . It is concluded that for the current tribosystem (AISI 52100 pin-on-disc samples lubricated by Shell NS-3 within the boundary regime), the properties of the tribofilms are affected by the test temperatures in the range of 80 to $150{ }^{\circ} \mathrm{C}$.

(1) The morphology and thickness of the tribofilm remain relatively constant with different testing temperatures. Inhomogeneous and pad-like tribofilms are observed, while the thicknesses are around 120 to $135 \mathrm{~nm}$.

(2) Generated at different temperatures, the tribofilms show a varying chemical composition along depth. The tribofilm generated at a higher temperature has higher Fe and lower $\mathrm{P}$ and Ca content.

(3) The mechanical properties of the tribofilms are affected by the temperature. The tribofilm formed at higher temperature show greater elastic modulus, hardness and yield strength. But by considering that all the mechanical properties are measured at room temperature, the difference may be overestimated.

\subsubsection{Friction mechanisms of the tribosystem}

The contributions of asperity deformation and surface adhesion forces to the total friction levels are investigated separately. The conclusions are summarized below:

(1) Friction caused by asperity deformation

A SAM-based single asperity sliding model was developed in Chapter $\mathbf{3}$ and used in Chapter 5 to investigate the friction contributed by asperity deformation without adhesion. The study is carried out by estimating the average asperity radius and interference depth, then calculating the frictional force by the developed model. 
It is found that in the current tribosystem, the COF levels contributed by the asperity deformation are around 0.005 to 0.015 , which are much smaller than the COFs observed in experiments.

(2) Friction caused by surface adhesion

The friction contributed by surface adhesion is investigated in this work. The shear strengths of the tribofilms are measured and reported in Chapter 2 . The real contact areas are studied in Chapter $\mathbf{4}$ by a SAM-based contact model. Sensitivity of real contact area on the input grid size is observed. The adhesion forces are calculated in Chapter 5.

Based on the calculation results, it is found that the adhesion force can explain the experimental results with fair accuracy. It is proposed that the adhesion force is the dominant friction in the current tribosystem, which is determined mainly by the mechanical properties of the tribofilm.

\subsection{Recommendations for future research}

(1) In this research, the properties of the tribofilms are measured at room temperatures. This may lead to inaccurate results, especially for the mechanical properties. Therefore, more research is proposed for the properties with respect to the tribofilm. In-situ measurements are generally preferable.

(2) The accuracy of the LFM can be further improved. In this research, a simple formula is used to link the vertical and lateral sensitivity of the AFM on the assumption that the PSPS is rotationally symmetric. More accurate results can be acquired by calibrating the lateral sensitivity of AFM by standard samples. 


\section{Appendix A}

\section{Elastic displacement and stress fields}

By employing Papkovich-Neuber potential and Fourier transformation, O'Sullivan and King [1] derived the displacement and stress fields in a layered material:

$$
\begin{gathered}
\widetilde{u}_{i}^{(k)}=\frac{1}{2 G_{k}} F T\left[\varphi_{, i}^{(k)}+x \psi_{1, i}^{(k)}+z_{k} \psi_{3, i}^{(k)}-\left(3-4 v_{k}\right) \psi_{i}^{(k)}\right] \\
\widetilde{\sigma}_{i}^{(k)}=F T\left[\varphi_{, i j}^{(k)}-2 v_{k}\left(\psi_{1,1}^{(k)}+\psi_{3,3}^{(k)}\right) \delta_{i j}-\left(1-2 v_{k}\right)\left(\psi_{i, j}^{(k)}+\psi_{j, i}^{(k)}\right)+x \psi_{1, i j}^{(k)}+\right. \\
\left.z_{k} \psi_{3, i j}^{(k)}\right]
\end{gathered}
$$

with:

$$
\begin{aligned}
& \widetilde{\varphi}^{(k)}=A^{(k)} e^{-\alpha z_{k}}+\bar{A}^{(k)} e^{\alpha z_{k}} \\
& \widetilde{\psi}_{1}^{(k)}=B^{(k)} e^{-\alpha z_{k}}+\bar{B}^{(k)} e^{\alpha z_{k}} \\
& \widetilde{\psi}_{3}^{(k)}=C^{(k)} e^{-\alpha z_{k}}+\bar{C}^{(k)} e^{\alpha z_{k}}
\end{aligned}
$$

( $k=1$ indicates coating material and $k=2$ indicates substrate material. $A, B$ and $C$ are coefficients to be determined by boundary conditions).

Once the frequency responses are acquired, the displacement and subsurface stress can then be linked to surface tractions by DC-FFT:

$$
\begin{aligned}
& {\left[\begin{array}{c}
u_{e}^{x} \\
u_{e}^{y} \\
u_{e}^{z}
\end{array}\right]=I F F T\left\{\left[\begin{array}{lll}
\widetilde{C}_{q_{x}}^{u_{x}} & \widetilde{C}_{q_{y}}^{u_{x}} & \widetilde{C}_{p}^{u_{x}} \\
\widetilde{C}_{q_{x}}^{u_{y}} & \widetilde{\boldsymbol{C}}_{q_{y}}^{u_{y}} & \widetilde{\boldsymbol{C}}_{p}^{u_{y}} \\
\widetilde{\boldsymbol{C}}_{q_{x}}^{u_{z}} & \widetilde{\boldsymbol{C}}_{q_{y}}^{u_{z}} & \widetilde{\boldsymbol{C}}_{p}^{u_{z}}
\end{array}\right] \cdot *\left[\begin{array}{c}
\widetilde{q}_{x} \\
\widetilde{q}_{y} \\
\widetilde{p}
\end{array}\right]\right\}} \\
& \boldsymbol{\sigma}_{\boldsymbol{e}}^{(\boldsymbol{k})}=\operatorname{IFFT}\left(\widetilde{\boldsymbol{C}}_{\boldsymbol{p}}^{\sigma^{(k)}} \cdot * \widetilde{\boldsymbol{p}}+\widetilde{\boldsymbol{C}}_{\boldsymbol{q}_{x}}^{\sigma^{(k)}} * \widetilde{\boldsymbol{q}}_{x}+\widetilde{\boldsymbol{C}}_{\boldsymbol{q}_{y}}^{\sigma^{(k)}} * * \widetilde{\boldsymbol{q}}_{y}\right)
\end{aligned}
$$

where $\boldsymbol{q}_{\boldsymbol{x}}$ is the surface traction in x direction, $\boldsymbol{q}_{\boldsymbol{y}}$ is the surface traction in y direction and $\boldsymbol{p}$ is the normal pressure. The tilde sign on top of variables stands for 2D Fourier transformation. 
By letting $\boldsymbol{q}_{\boldsymbol{x}}$ and $\boldsymbol{q}_{\boldsymbol{y}}$ equal to zero in Eq. (3), the expressions of $\boldsymbol{u}_{\boldsymbol{e}}(p)$ can be acquired.

\section{Residual displacement and stress fields}

The SAM is used in this work to evaluate the residual displacement and stress fields. In SAM, the plastic strains are considered as "eigenstrains", while their influence on the whole domain is solved by a combination of analytical and numerical approaches. The method proposed recently by Wang et al. [2] is used in this work, which is described briefly below.

Firstly, a simple case is introduced, see Fig. 1. A full-space is composed by two halfspaces I and II. These two half-spaces are perfectly bonded, while eigenstrains exist in half-space $I$.

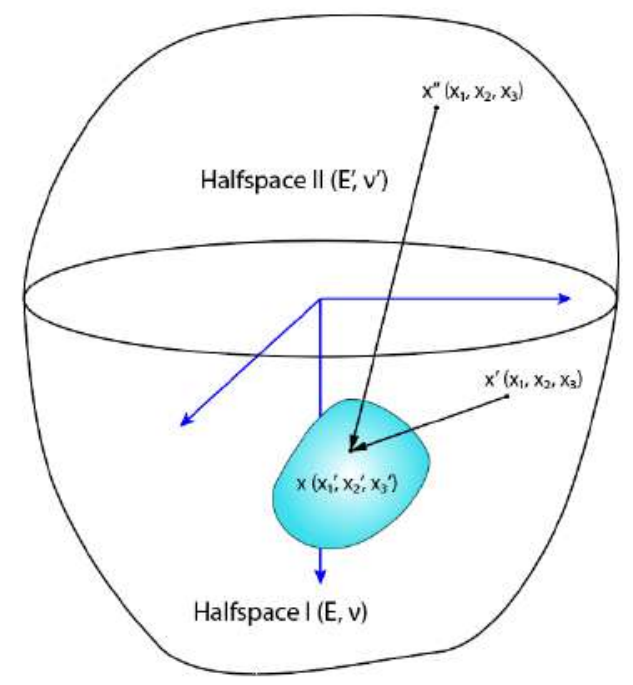

Fig. 1. Two joined elastic half-spaces inclusion problem, where two half-spaces are perfectly bonded together and with inclusions in the half-space I.

The stress and displacement fields in both half spaces are solved. For example, the displacement field in half space I can be expressed as: 


$$
\begin{aligned}
& 2 \mu u_{i j}^{I, o u t}\left(x_{1 \alpha}, x_{2 \beta}, x_{3 \gamma}\right) / C \\
& =\sum_{v=1}^{L} \sum_{\eta=1}^{M} \sum_{\xi=1}^{N} U_{i j k l}^{(0)}\left(x_{1 \alpha}-x_{1 \xi}, x_{2 \beta}-x_{2 \eta}, x_{3 \gamma}\right. \\
& \left.-x_{3 v}\right) e_{k l}\left(x_{1 \xi}, x_{2 \eta}, x_{3 v}\right) \\
& +\sum_{v=1}^{L} \sum_{\eta=1}^{M} \sum_{\xi=1}^{N} U_{i j k l}^{(1)}\left(x_{1 \alpha}-x_{1 \xi}, x_{2 \beta}-x_{2 \eta}, x_{3 \gamma}\right. \\
& \left.+x_{3 v}\right) e_{k l}\left(x_{1 \xi}, x_{2 \eta}, x_{3 v}\right) \\
& +x_{3 v} \sum_{v=1}^{L} \sum_{\eta=1}^{M} \sum_{\xi=1}^{N} U_{i j k l}^{(5)}\left(x_{1 \alpha}-x_{1 \xi}, x_{2 \beta}-x_{2 \eta}, x_{3 \gamma}\right. \\
& \left.+x_{3 v}\right) e_{k l}\left(x_{1 \xi}, x_{2 \eta}, x_{3 v}\right) \\
& +x_{3 v}^{2} \sum_{v=1}^{L} \sum_{\eta=1}^{M} \sum_{\xi=1}^{N} U_{i j k l}^{(5)}\left(x_{1 \alpha}-x_{1 \xi}, x_{2 \beta}-x_{2 \eta}, x_{3 \gamma}\right. \\
& \left.+x_{3 v}\right) e_{k l}\left(x_{1 \xi}, x_{2 \eta}, x_{3 v}\right) \\
& \left(\mathbf{1} \leq \alpha \leq M, 1 \leq \beta \leq N, 1 \leq \gamma \leq L_{1}\right)
\end{aligned}
$$

Readers are referred to Wang's work for more details on the influence matrices and coefficients.

\section{References}

[1] O'Sullivan, T. C., \& King, R. B. (1988). Sliding contact stress field due to a spherical indenter on a layered elastic half-space. Journal of tribology, 110(2), 235-240.

[2] Wang, Z., Yu, H., \& Wang, Q. (2016). Analytical solutions for elastic fields caused by eigenstrains in two joined and perfectly bonded half-spaces and related problems. International Journal of Plasticity, 76, 1-28. 

Part B 



\section{Paper A}

Wang, C., \& Schipper, D. J. (2018). On an elastoplastic sliding model for a coated single asperity. Lubricants, 6(4), 96. 



\title{
On an Elastoplastic Sliding Model for a Coated Single
}

\author{
Asperity \\ Can Wang * and Dik J. Schipper \\ Laboratory for Surface Technology and Tribology, Department of Engineering Technology, \\ University of Twente, 7522 NB Enschede, The Netherlands \\ *Correspondence: c.wang-1@utwente.nl
}

\begin{abstract}
In this study, a sliding friction model for coated single asperity contacts is proposed. A displacement-driven layered contact algorithm is firstly introduced and verified by the finite element method. Then, this algorithm is applied to simulate the contact between two semispherical asperities. The full sliding contact process is discretized into a series of transient steps, and each of these steps are calculated by the displacement-driven contact algorithm. The effects of the interference depth and the properties of, respectively, the tribofilm (thickness, elastic modulus, and yield strength) and the nanocrystalline layer on the sliding coefficient of friction are investigated. The results suggest that when surface adhesion and asperity damage are ignored, the plastic deformation of the tribofilm is the main source of the sliding friction. Greater interference depth, tribofilm with greater thickness, higher elastic modulus or lower yield strength, and the presence of a nanocrystalline layer will lead to a higher coefficient of friction in single asperity sliding.
\end{abstract}

Keywords: boundary lubrication; friction model; tribofilm

\section{Introduction}

Sliding friction under boundary lubrication has been studied for almost a century [1][5]. In the boundary lubrication regime, the lubricant film is not sufficiently thick to separate the surfaces and solid-to-solid contact will occur. Under these conditions, primarily, the highest peaks (asperities) on the surfaces will come into contact. 
Therefore, the asperities are carrying the load locally, so on a microscale, the stresses exceed the yield of the material causing plastic flow even though the macroscopic pressure is well below the yield limit. This elastoplastic deformation of asperities has been regarded as one of the important sources of friction [2]. Meanwhile, a solid layer is also generated in boundary lubrication and plays a crucial role in protecting the system against severe friction and wear. This layer consists of two separate layers, one produced by chemical reactions and one by the mechanical energy dissipated at the top layer of the bulk material (nanocrystalline (NC) layer) (see Fig.1). The properties and composition of these layers are dominated by the lubricants used (mainly the additives package) and the conditions under which the system is running. Using this representation of the system in previous publications, wear rates were successfully predicted [6][7]. In these publications, mechanical properties available in the literature were used for both the NC layer and the tribofilm. However, the coefficient of friction was presumed known and constant. In this publication, the latter will be investigated in more detail and the main question to be answered is: "Can plastic deformation of the tribofilm and NC layer explain the coefficient of friction observed in boundary lubrication?"

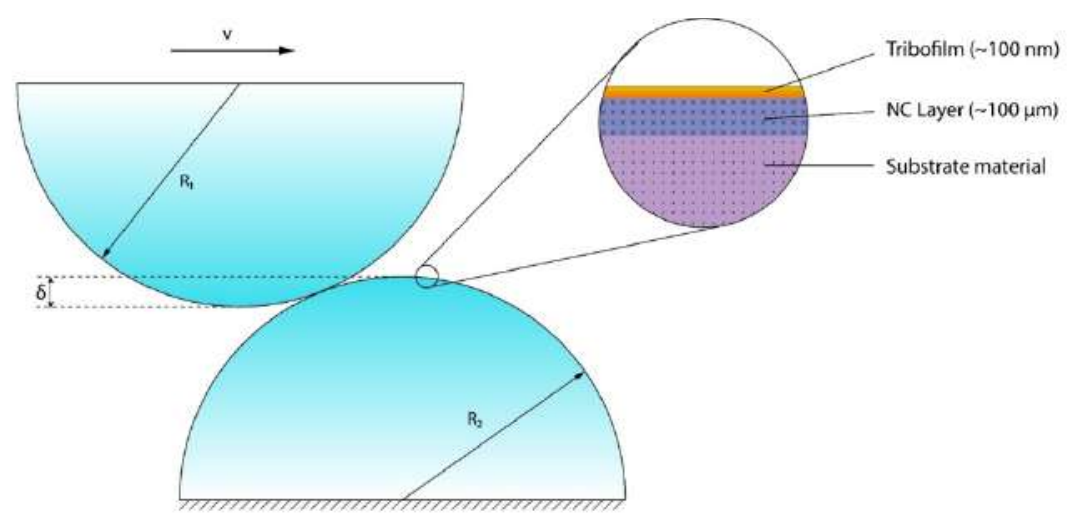

Fig. 1. Layers present in a sliding asperity contact system. The top layer is the tribofilm, which is a mixture of oxides and chemical products of the lubricant. The second layer is a nanocrystalline layer formed at the top of the bulk material by severe plastic deformation under high hydrostatic pressure and high shear rates.

It has been known for many years that different additives influence the tribological behavior of a system (the level of friction and wear). This can be explained by the 
fact that different additives will produce different tribofilms [8] which tweak the contact status (contact pressure and deformation, real contact area, etc.) of asperities. The finite element method (FEM) is a commonly used approach in solving contact problems. Liu et al. [9] analyzed the elastic-plastic contact of rough surfaces in line-contact problems. This work was extended by Etsion [10] for point contacts. Furthermore, Jackson et al. [11] developed a finite element model predicting the normal and tangential resisting forces of two sliding elastic, perfectly plastic frictionless spherical asperities. Mulvihill et al. [12] improved this work by imposing greater interference depths and considering surface adhesion in the contact. However, it should be noted that performing FEM contact analysis is time consuming since a very fine mesh is needed in contact areas to ensure convergence and accurate results, while the domain needs to be large enough to avoid edge effects. In addition, the contact condition itself (no penetration of the two bodies) is not inherently incorporated to the FEM method and requires additional degrees of freedom (DOF) for a solution to be reached. Often, the penalty function is used, in which the magnitude of the contact stiffness is tuned using a Hertzian reference contact. While the obtained value may be true for the reference case, it is very possible that in different scenarios the values obtained do not hold.

In addition to FEM, which is governed by continuum mechanics, alternative options have been proposed to solve the contact process. Molecular dynamics (MD), for example, simulates the contact process at the atomic level. This method allows all atoms inside the calculation domain to move simultaneously, and interactions between these atoms are calculated by Newton's law. It is thus possible to track the position information of each atom at a given time. Therefore, MD is particularly suitable for solving contact mechanics at the nanoscale [13][14][15]. However, with increasing size and time scale, the computational cost will become unacceptably high and limit the application of MD in contact simulation. In recent decades, the discrete element method (DEM), as another alternative, has gained popularity in numerical tribology. DEM considers that the contact areas are composed of heterogeneous particles interacting with each other, governed by conservation and dynamics equations. DEM can calculate the behavior of detached particles efficiently and therefore is widely applied in simulating the third body behavior [16][17] and wear process [18][19]. DEM became even more versatile by combining with the cellular automata method: a series of dry friction problems between different materials were successfully simulated [20][21][22]. However, properly choosing the size of single particles and defining the interaction force between them are a challenge [23]. 
Besides, the application of DEM is also constrained by computational cost in large scale and a longer time period.

The fast Fourier transform (FFT) technique and semianalytical method (SAM) provide an alternative for solving elastic and elastoplastic contact more efficiently. Various problems including single and layered material contact have been solved by SAM [24]. Also, techniques have been developed to accelerate the calculation and improve the accuracy. Polonsky and Keer [25] proposed an iterative scheme by applying the conjugate gradient method. Liu et al. [26] eliminated the periodic errors in regular FFT by introducing the discrete fast Fourier transform (DC-FFT) technique. However, the aforementioned works only handled purely elastic contacts. To solve elastoplastic contact problems, Jacq et al. [27] presented a semianalytical method by considering plastic deformation as eigenstrains, based on Chiu's results [28][29]. Recently, Wang et al. [30] acquired stress and displacement fields of eigenstrains in two perfectly bonded layered halfspaces, making it possible to solve the elastoplastic contact of layered material.

In this paper, the sliding friction of a single asperity contact including a tribofilm and a nanocrystalline layer is solved by applying the DC-FFT technique and SAM based on the work in [22]. Consequently, this work extends the model proposed by Boucly et al. [31] which involved only homogeneous material, to a layered system. The plasticity in the layered structure is considered to follow the $\mathrm{J} 2$ flow theory [32] and is solved by the method in reference [30]. Green's assumption on steady state sliding [33] is applied: two asperities will not approach or separate over each other while sliding, e.g., a predefined separation of the two surfaces is given. To get an initial indication of the influence of the different aspects affecting friction, the effects of mechanical properties of the tribofilm (elastic modulus, Poisson's ratio, yield strength, thickness), interference depth, and nanocrystalline layer are studied separately. The adhesion between tribofilms and damage to asperities are ignored, as the main goal of the study is to investigate the contribution of plastic deformation to friction.

\section{Theory and Methodology}

In this section, the general model is discussed first. The main backbone is a displacement-driven contact model for a layered elastoplastic system. This general 
model is used to investigate a single asperity contact with different mechanical properties.

\subsection{Displacement-Driven Contact Model}

First, a reference coordinate system is introduced, as shown in Fig. 2. For simplicity but without loss of generality, a contact of an elastoplastic object loading on a rigid flat is considered,

where:

$p$ is the contact pressure distribution built within the contact area;

$e_{p}$ is the plastic strain in the elastoplastic object formed due to contact stress;

$u_{e}$ is the surface displacement due to contact pressure, a function of the contact pressure distribution $(p)$;

$u_{r}$ is the surface displacement due to residual plastic strain, a function of plastic strains $\left(e_{p}\right)$;

$h_{0}$ is the initial surface separation relative to the initial undeformed geometry;

$\delta_{z}$ is the rigid body movement.

The initial geometry $\left(h_{0}\right)$ of contact objects (and their mechanical properties), together with a rigid body movement $\left(\delta_{z}\right)$, serves as input for the model. The model should be able to predict the contact pressure $(p)$ and the subsurface plastic strains $\left(e_{p}\right)$. 


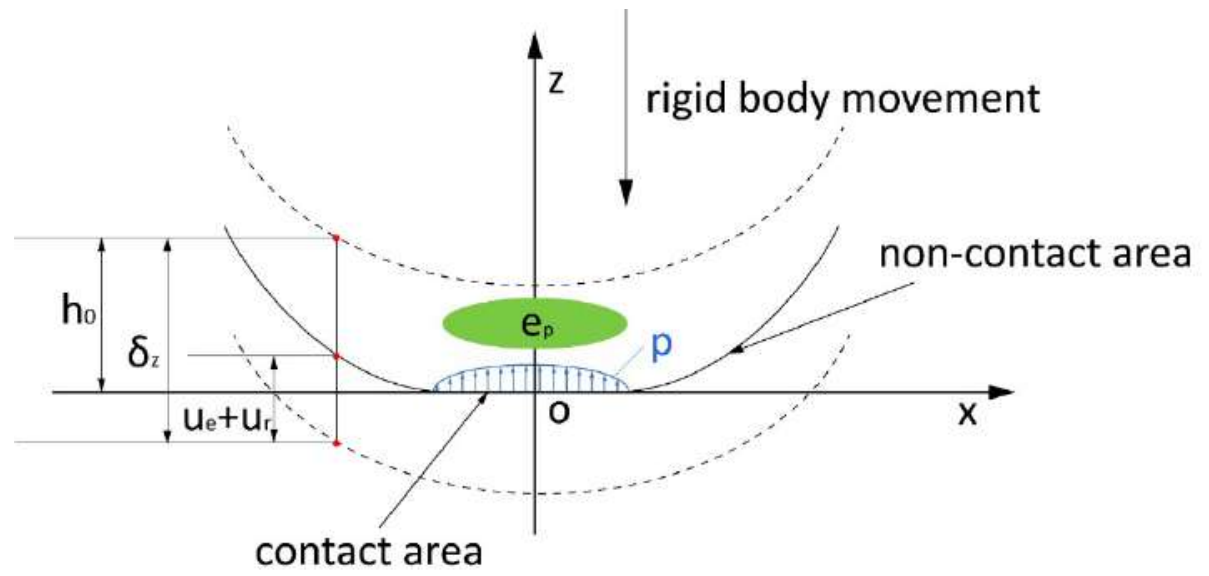

Fig. 2. Schematic of the contact.

In the whole domain in which contact and noncontact areas both exist, the following equations and conditions hold:

Inside the contact area:

$$
\begin{gathered}
\boldsymbol{u}_{\boldsymbol{e}}(\boldsymbol{p})+\boldsymbol{u}_{r}\left(\boldsymbol{e}_{\boldsymbol{p}}\right)+\boldsymbol{h}_{\mathbf{0}}=\boldsymbol{\delta}_{\mathrm{z}} \\
\boldsymbol{p}>\mathbf{0}
\end{gathered}
$$

Outside the contact area:

$$
\begin{gathered}
\boldsymbol{u}_{\boldsymbol{e}}(\boldsymbol{p})+\boldsymbol{u}_{r}\left(\boldsymbol{e}_{\boldsymbol{p}}\right)+\boldsymbol{h}_{\mathbf{0}} \geq \boldsymbol{\delta}_{\mathrm{z}} \\
\boldsymbol{p}=\mathbf{0}
\end{gathered}
$$


Eq. (1) and (2) indicate that in contact areas, surface separation must be zero (no penetration). In noncontact areas, however, surface separation should be greater than zero, which is shown in Eq. (3) and (4).

Since the ultimate goal of the model is to find the pressure distribution and plastic strains that make Eq. (1)-(4) hold, the exact expressions of $u_{e}(p)$ and $u_{r}\left(e_{p}\right)$ should be built first. This will be described in the following subsections.

\subsubsection{Elastic Displacement and Stress Fields}

By employing Papkovich-Neuber potential and Fourier transformation, O'Sullivan and King [34] derived the displacement and stress fields in a layered material:

$$
\begin{gathered}
\widetilde{u}_{i}^{(k)}=\frac{1}{2 G_{k}} F T\left[\varphi_{, i}^{(k)}+x \psi_{1, i}^{(k)}+z_{k} \psi_{3, i}^{(k)}-\left(3-4 v_{k}\right) \psi_{i}^{(k)}\right] \\
\widetilde{\sigma}_{i}^{(k)}=\boldsymbol{F T}\left[\varphi_{, i j}^{(k)}-2 v_{k}\left(\psi_{1,1}^{(k)}+\psi_{3,3}^{(k)}\right) \delta_{i j}-\left(1-2 v_{k}\right)\left(\psi_{i, j}^{(k)}+\psi_{j, i}^{(k)}\right)+x \psi_{1, i j}^{(k)}+\right. \\
\left.z_{k} \psi_{3, i j}^{(k)}\right]
\end{gathered}
$$

with:

$$
\begin{aligned}
& \widetilde{\boldsymbol{\varphi}}^{(k)}=\boldsymbol{A}^{(k)} \boldsymbol{e}^{-\alpha z_{k}}+\overline{\boldsymbol{A}}^{(k)} \boldsymbol{e}^{\alpha z_{k}} \\
& \widetilde{\boldsymbol{\psi}}_{1}^{(k)}=\boldsymbol{B}^{(k)} \boldsymbol{e}^{-\alpha z_{k}}+\bar{B}^{(k)} \boldsymbol{e}^{\alpha z_{k}} \\
& \widetilde{\boldsymbol{\psi}}_{3}^{(k)}=\boldsymbol{C}^{(k)} \boldsymbol{e}^{-\alpha z_{k}}+\overline{\boldsymbol{C}}^{(k)} \boldsymbol{e}^{\alpha z_{k}}
\end{aligned}
$$

where $k=1$ indicates coating material and $k=2$ indicates substrate material. $A, B$, and $C$ are coefficients to be determined by boundary conditions (see [34] for more details).

Once the frequency responses are acquired, the displacement and subsurface stress can then be linked to surface tractions by DC-FFT [26]: 


$$
\begin{aligned}
& {\left[\begin{array}{l}
u_{e}^{x} \\
u_{e}^{y} \\
u_{e}^{z}
\end{array}\right]=I F F T\left\{\left[\begin{array}{ccc}
\widetilde{C}_{q_{x}}^{u_{x}} & \widetilde{\boldsymbol{C}}_{q_{y}}^{u_{x}} & \widetilde{\boldsymbol{C}}_{p}^{u_{x}} \\
\widetilde{\boldsymbol{C}}_{\boldsymbol{q}_{y}}^{u_{y}} & \widetilde{\boldsymbol{C}}_{\boldsymbol{q}_{y}}^{u_{y}} & \widetilde{\boldsymbol{C}}_{p}^{u_{y}} \\
\widetilde{\boldsymbol{C}}_{\boldsymbol{q}_{x}}^{u_{z}} & \widetilde{\boldsymbol{C}}_{\boldsymbol{q}_{y}}^{u_{z}} & \widetilde{\boldsymbol{C}}_{p}^{u_{z}}
\end{array}\right] * *\left[\begin{array}{c}
\widetilde{\boldsymbol{q}}_{x} \\
\widetilde{\boldsymbol{q}}_{y} \\
\widetilde{\boldsymbol{p}}
\end{array}\right]\right\}}
\end{aligned}
$$

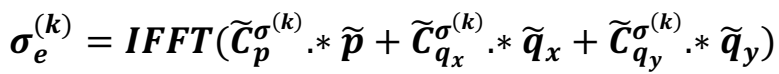

where $q_{x}$ is the surface traction in $x$ direction, $q_{y}$ is the surface traction in y direction, and $p$ is the normal pressure. The tilde sign on top of variables stands for 2D Fourier transformation.

By letting $q_{x}$ and $q_{y}$ equal zero in Eq. (7), the expressions of $u_{e}(p)$ can be acquired.

\subsubsection{Residual Displacement and Stress Fields}

The SAM was used in this work to evaluate the residual displacement and stress fields. In SAM, the plastic strains are considered as "eigenstrains", while their influence on the domain as a whole is solved by a combination of analytical and numerical approaches. The method proposed recently by Wang et al. [30] was used in this work and is described briefly below.

Firstly, a simple case is introduced, see Fig. 3 [30]. A full space is composed of two halfspaces I and II. These two halfspaces are perfectly bonded, while eigenstrains exist in halfspace $\mathrm{I}$. 


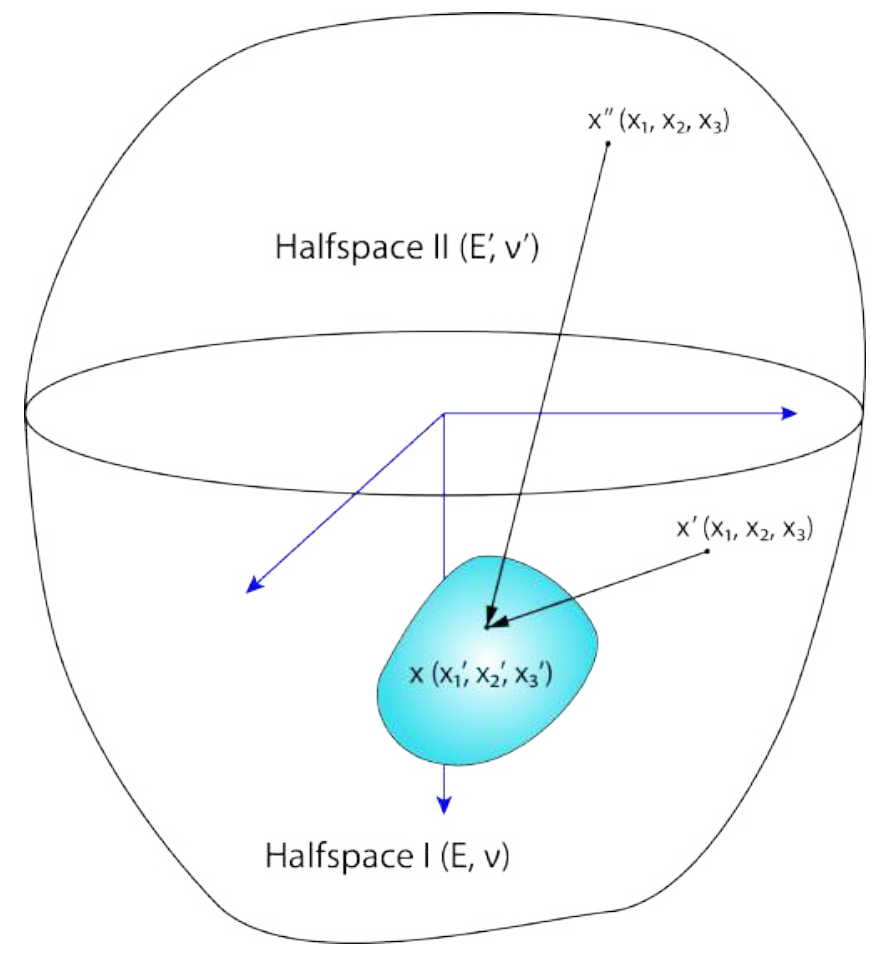

Fig. 3. Schematic of two joined elastic halfspaces inclusion problem, where two halfspaces are perfectly bonded together with inclusions in halfspace I [30].

The stress and displacement field in both halfspaces are solved. For example, the displacement field in halfspace I can be expressed as 


$$
\begin{aligned}
2 \mu u_{i j}^{I, \text { out }}\left(x_{1 \alpha}, x_{2 \beta}, x_{3 \gamma}\right) / C & \\
= & \sum_{v=1}^{L} \sum_{\eta=1}^{M} \sum_{\xi=1}^{N} U_{i j k l}^{(0)}\left(x_{1 \alpha}-x_{1 \xi}, x_{2 \beta}-x_{2 \eta}, x_{3 \gamma}-x_{3 v}\right) e_{k l}\left(x_{1 \xi}, x_{2 \eta}, x_{3 v}\right) \\
& +\sum_{v=1}^{L} \sum_{\eta=1}^{M} \sum_{\xi=1}^{N} U_{i j k l}^{(1)}\left(x_{1 \alpha}-x_{1 \xi}, x_{2 \beta}-x_{2 \eta}, x_{3 \gamma}+x_{3 v}\right) e_{k l}\left(x_{1 \xi}, x_{2 \eta}, x_{3 v}\right) \\
+ & x_{3 v} \sum_{v=1}^{L} \sum_{\eta=1}^{M} \sum_{\xi=1}^{N} U_{i j k l}^{(5)}\left(x_{1 \alpha}-x_{1 \xi}, x_{2 \beta}-x_{2 \eta}, x_{3 \gamma}+x_{3 v}\right) e_{k l}\left(x_{1 \xi}, x_{2 \eta}, x_{3 v}\right) \\
+ & x_{3 v}^{2} \sum_{v=1}^{L} \sum_{\eta=1}^{M} \sum_{\xi=1}^{N} U_{i j k l}^{(5)}\left(x_{1 \alpha}-x_{1 \xi}, x_{2 \beta}-x_{2 \eta}, x_{3 \gamma}+x_{3 v}\right) e_{k l}\left(x_{1 \xi}, x_{2 \eta}, x_{3 v}\right) \\
& (1 \leq \alpha \leq M, 1 \leq \beta \leq N, 1 \leq \gamma \leq L) .
\end{aligned}
$$

Readers are referred to [30] for more details on the influence matrices/coefficients.

However, this simple case solution is not instantly applicable to the model because in layered structure contact problems, a halfspace is needed instead of a full space, as shown in Fig. 4a. This halfspace is composed of a fully extended halfspace (the substrate) and a finite halfspace (the tribofilm, due to its finite thickness).

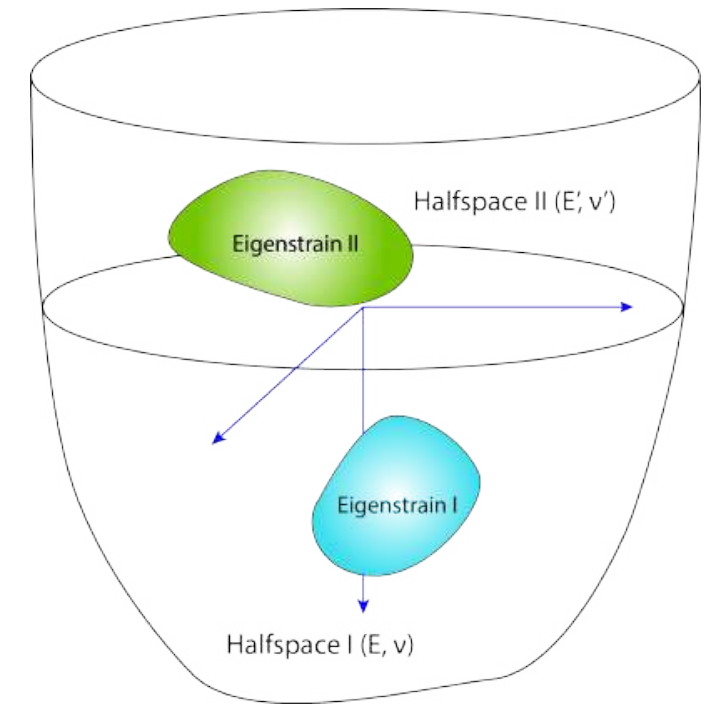

(a) Schematic of layered halfspace and eigenstrains 


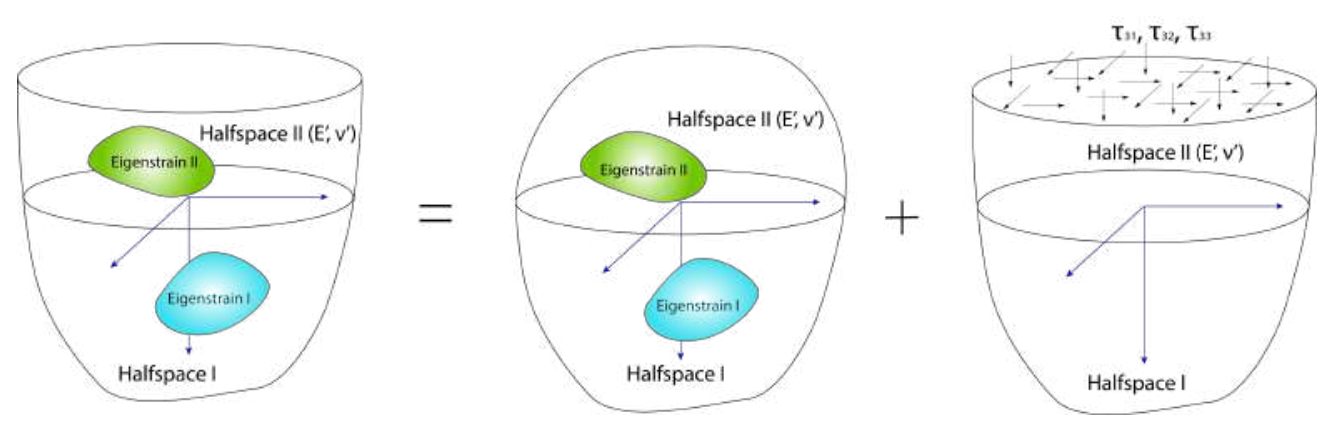

(b) Layered contact problem subproblems I and II

Fig. 4. Schematic of the superposition method for solving a layered structure [30].

The principle of superposition states that for a given small deformation problem domain, if the state $I$ is a solution to the fundamental elasticity equations with prescribed body forces $F_{1}$ and surface tractions $T_{1}$, and state II is a solution to the fundamental equations with prescribed body forces $F_{\|}$and surface tractions $T_{\| 1}$, then state I + II will be a solution to the problem with body forces $F_{I}+F_{\| I}$ and surface tractions $T_{1}+T_{\|}$. Therefore, the layered structure contact problem can be split into two subproblems: an eigenstrain problem in a full space plus a set of imaginary surface tractions to make the aggregate solution satisfy the halfspace boundary conditions (surface traction free), as seen in Fig. $\mathbf{4 b}$.

Note that the first subproblem is the eigenstrain problem in a full space, which can be solved by the method mentioned in Eq. (9). The second subproblem, however, is a surface traction problem, which can be solved by Eq. (7) and (8), by letting $q_{x}, q_{y}$, and $p$ equal the imaginary surface tractions. By summing up the displacements caused by the eigenstrains and the imaginary stresses, the expressions of $u_{r}\left(e_{p}\right)$ are acquired.

\subsubsection{Model Route}

With $u_{e}(p)$ and $u_{r}\left(e_{p}\right)$ being expressed, the pressure distribution $p$ and plastic strains $e_{p}$ are now explicitly linked to the inputs (rigid body movement $\delta_{z}$ and initial surface geometry $h_{0}$ ) by Eq. (1) to (4). This is in essence an $M^{*} N$ linear system. However, it should be noted that the subsurface stress generated by the surface contact pressure causes plastic strains. These plastic strains cause residual displacement and stresses, which in turn alter the geometry and change the surface contact pressure. In other words, $p$ and $e_{p}$ are coupled. Thus, a direct solution is difficult to acquire. 
Therefore, an iteration loop is proposed to address the problem. First, an initial status is set. The pressure and subsurface stress are solved first, followed by a radial return algorithm to determine plastic deformation for the current loop. Then, the residual displacement is calculated and surface geometry is updated. Afterwards, the pressure distribution is recalculated based on the new surface geometry. This loop will keep running till a convergence in pressure distribution is achieved (see Fig. 5). Note that in each iteration, an inner iteration (return mapping) is needed to map the stress back to yield surface and update plastic deformation. This process is shown in Fig. 6.

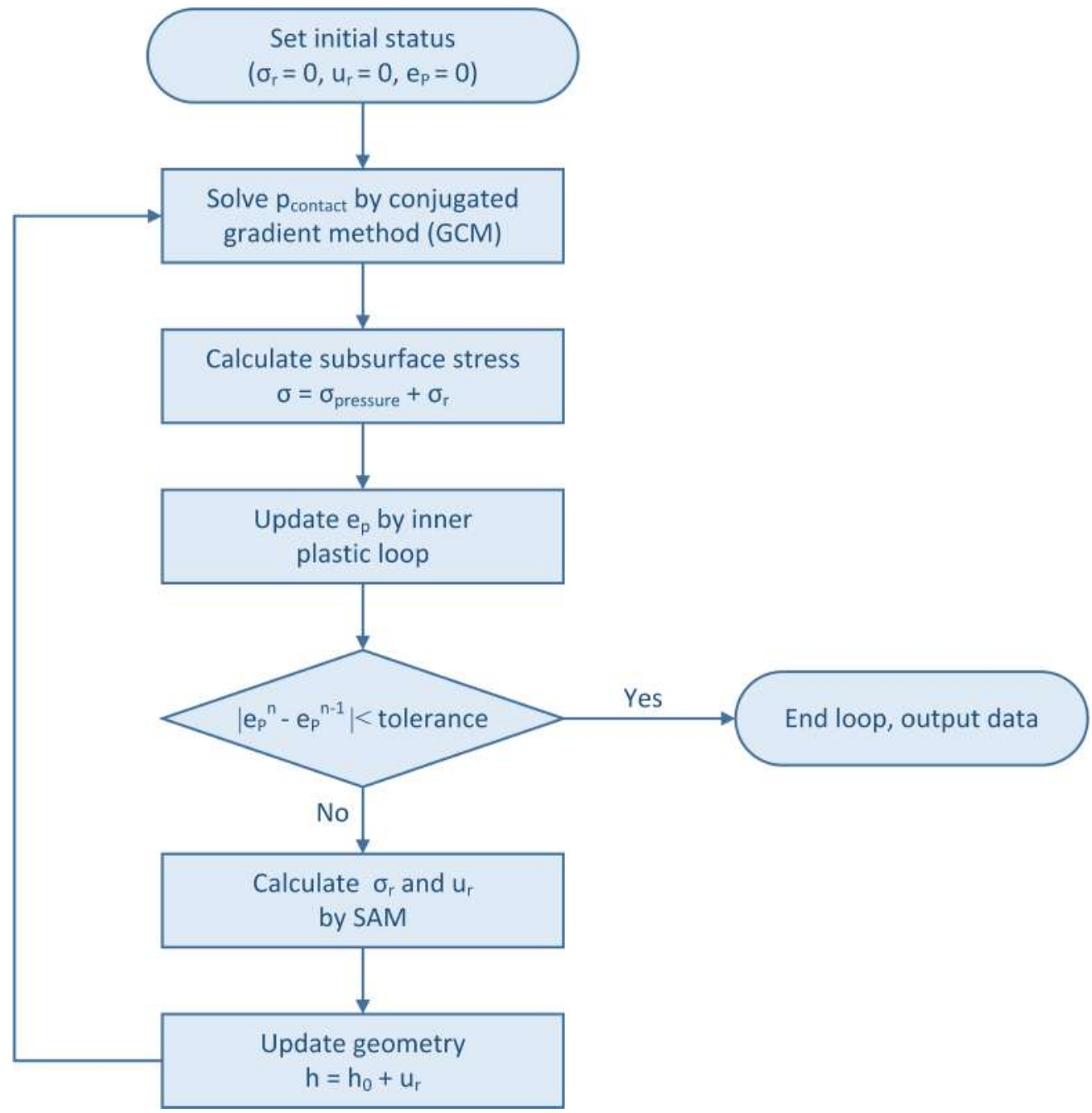

Fig. 5. Flowchart of iteration loop. 


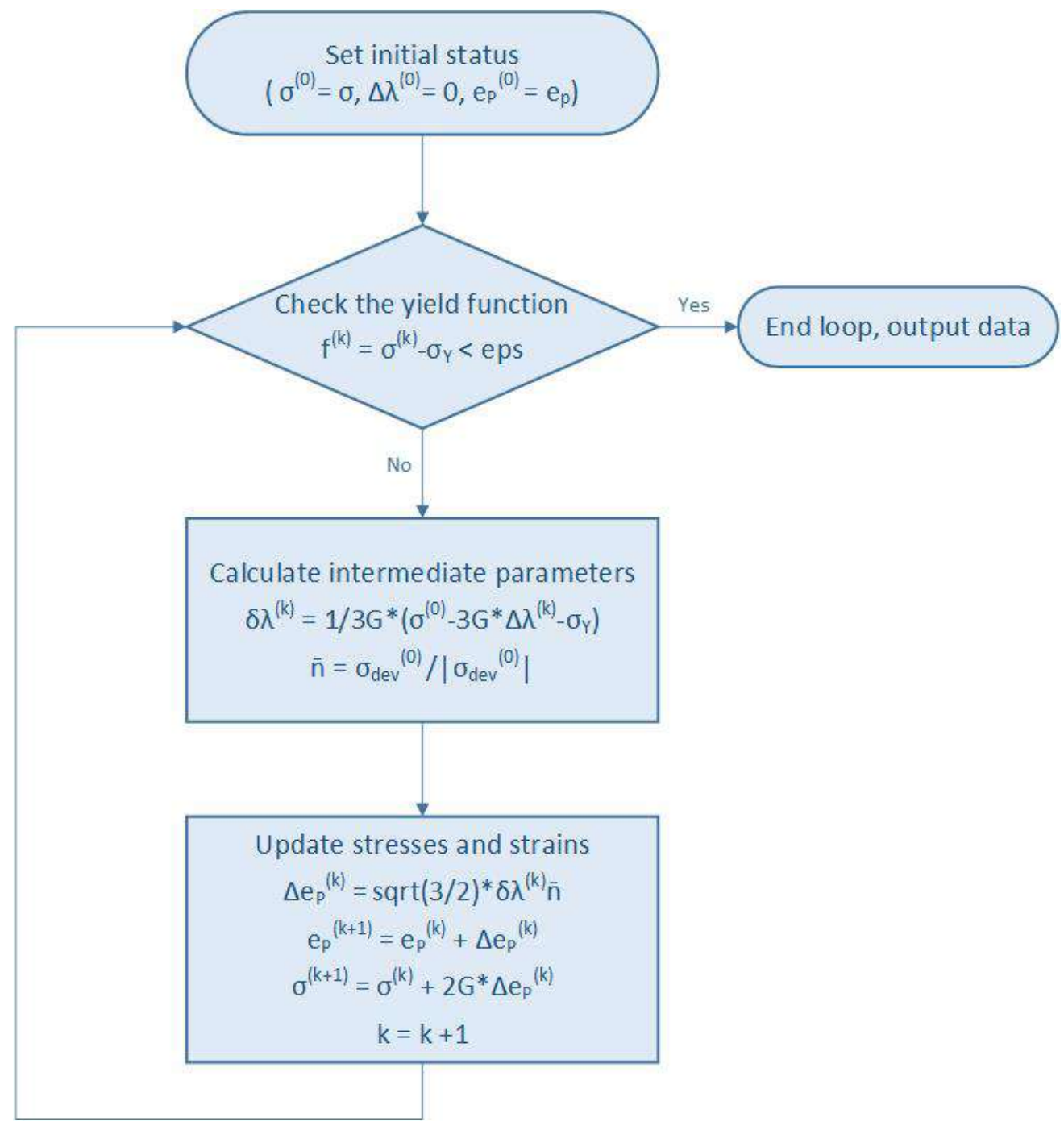

Fig. 6. Inner plastic loop.

\subsection{Single Asperity Sliding}

In this work, the sliding asperity contact was idealized as a normal contact between two hemispheres, following the work of Boucly [31] (see Fig. 7). Using a hemisphere to represent the asperity is a commonly adopted approach when studying a single asperity contact, as it allows a relatively simple calculation of the contact parameters. The two hemispheres are aligned with the distance between two basis planes being held constant as $h$, while the interference depth is $d_{0}$. Elastoplastic 
behavior of both tribofilm and substrate materials are considered, while adhesion between the surfaces is ignored.

The whole contact process is calculated in an incremental manner. In each incremental step, the corresponding rigid body movement is determined first, then the tangential and normal forces generated by the sliding contact, which are regarded as frictional and normal force, are calculated by the process proposed in Section 2.1 .
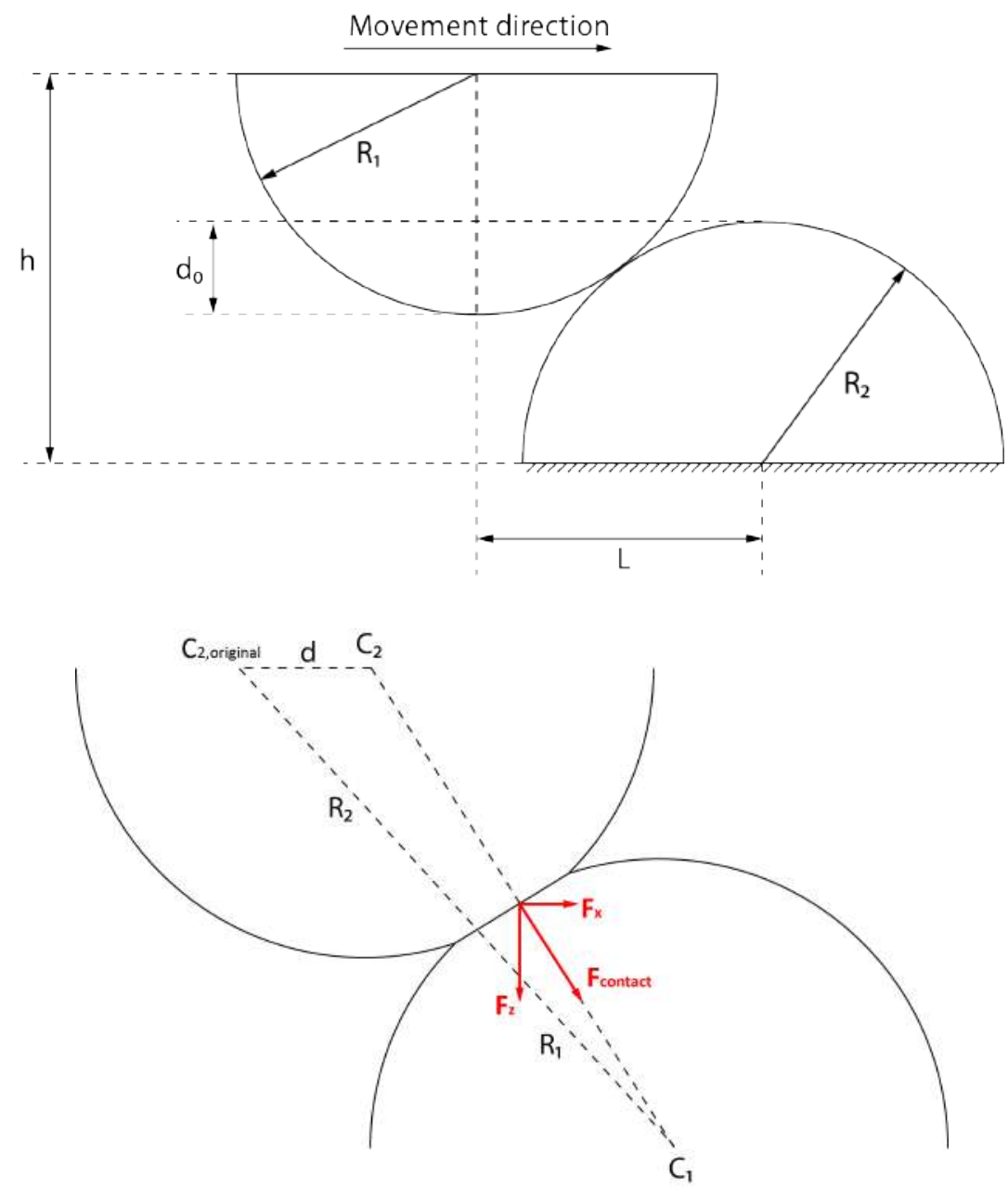

Fig. 7. Sliding contact between two asperities [20]. 
First, the whole sliding contact process is discretized into $n$ equally distributed incremental steps. In each of these incremental steps, the rigid body movement in the contact direction can be derived. In the ith step, note that in the triangle $C_{2, \text { originar }}$ $C_{1}-C_{2}$, the distance between the two centers of the hemispheres is $C_{1} C_{2}$, Therefore, the rigid body movement is

$$
\delta_{z, i}=2 * R-C_{1} C_{2, i} .
$$

With $\boldsymbol{\delta}_{z, i}$ calculated, the contact force in each step $F_{\text {contact,i }}$ can be calculated by the displacement-driven contact model. The frictional force and normal force in each step then can be acquired as tangential and vertical components of $F_{\text {contact, } i:}$

$$
\begin{aligned}
& \boldsymbol{F}_{\text {friction } \_i}=\boldsymbol{F}_{\text {contact }, i_{-} x}=\iint_{\Omega \in \operatorname{contact} \text { area }} \boldsymbol{p} * \boldsymbol{d} \Omega * \cos \theta \\
& \boldsymbol{F}_{\text {normal } \_i}=\boldsymbol{F}_{\text {contact, } i \_y}=\iint_{\Omega \in \text { contact area }} \boldsymbol{p} * d \Omega * \sin \theta
\end{aligned}
$$

where $\theta$ is the angle between $d \Omega$ and the horizontal plane.

For the whole sliding process, the coefficient of friction (COF) is defined as

$$
C O F=\frac{\sum F_{\text {friction_i } i}}{\sum \boldsymbol{F}_{\text {normal_i } i}}=\frac{\sum \boldsymbol{F}_{\text {contact }, i \_x}}{\sum \boldsymbol{F}_{\text {contact }, i \_y}}
$$

Readers are referred to Boucly's work [31] for detailed expressions.

\section{Results and Discussion}


In this section, a tribosystem of a pair of steel asperities (25MnCr5) with a ZDDP tribofilm is set as a baseline, which is commonly seen as a soft-tribofilm and hardsubstrate combination in industrial applications. The properties of the ZDDP film, steel substrate, and asperity radius were collected from the literature and are listed in Tab. 1.

Tab. 1. Properties of ZDDP tribofilm and steel asperities.

\begin{tabular}{cc}
\hline Parameter & Values \\
\hline Young's modulus, EZDDP $(\mathrm{GPa})$ & $35[35]$ \\
Poisson's ratio, VZDDP & 0.3 \\
Yield stress, $\sigma_{\text {ZDDP }}(\mathrm{MPa})$ & $500[35]$ \\
Young's modulus, Easperity $(\mathrm{GPa})$ & 205 \\
Poisson's ratio, $v_{\text {asperity }}$ & 0.3 \\
Yield stress, $\sigma_{\text {asperity }}(\mathrm{MPa})$ & 1200 \\
Radius of asperity $(\mu \mathrm{m})$ & $10[36]$ \\
\hline
\end{tabular}

Note: the Poission's ratio and yield strength of ZDDP film have not yet been reported in the literature. The yield strength above is estimated as one-third of the hardness in [35]. Poission's ratio is assumed to be 0.3 .

Next, a verification of displacement-driven contact model is presented, then the effects of different tribofilm properties on sliding friction are quantified.

\subsection{Verification of Displacement-Driven Contact Model}

The displacement-driven contact model proposed in Section $\mathbf{2 . 1}$ was verified by an asperity indented on a coated flat plane. For simplicity without losing generality, the asperity was assumed rigid, while both materials of the coated flat plane showed elastic-perfectly plastic behavior. The material properties and contact conditions are listed below in Tab. 2. Two different yield strengths of the coating material were used for comparison. The solution domain was meshed into an $80 \times 80 \times 80$ grid (20 grid over the thickness direction of tribofilm). With a single element of size $6 \times 6 \times 6$ $\mathrm{nm}$, this resulted a calculation domain sized $480 \times 480 \times 480 \mathrm{~nm}$, which was large enough to allow the plastic deformation to fully extend. 
Tab. 2. Parameters of the indentation model.

\begin{tabular}{cc}
\hline Parameter & Values \\
\hline Young's modulus, $\mathrm{E}_{\text {coating }}(\mathrm{GPa})$ & 35 \\
Poisson's ratio, $v_{\text {coating }}$ & 0.3 \\
Yield stress, $\sigma_{\text {coating }}(\mathrm{MPa})$ & 500,800 \\
Young's modulus, $\mathrm{E}_{\text {substrate }}(\mathrm{GPa})$ & 205 \\
Poisson's ratio, $v_{\text {substrate }}$ & 0.3 \\
Yield stress, $\sigma_{\text {substrate }}(\mathrm{MPa})$ & 1200 \\
Radius of asperity $(\mu \mathrm{m})$ & 10 \\
\hline
\end{tabular}

The results of the SAM model were verified by comparing the solutions with those from commercial FEM software (ABAQUS, from Dassault Systèmes, VélizyVillacoublay, France). The FEM model used four-node bilinear axisymmetric quadrilateral with full integration (type CAX4) elements. The element sizes varied from near the contact area (indicating the volume which has this fine mesh, $6 \times 6$ $\mathrm{nm}$ ) to $100 \times 100 \mathrm{~nm}$ in faraway areas. To limit the effect of boundary conditions in the FEM simulation, the modelling domain had to be at least 10 times larger than the contact zone. This gives a total of 55,214 elements in the FEM model.

A comparison of the results is presented in Fig. 8. The von Mises stress and equivalent plastic strain as a function of depth at the center of the contact are shown. Despite some minor differences, good agreement is observed. 


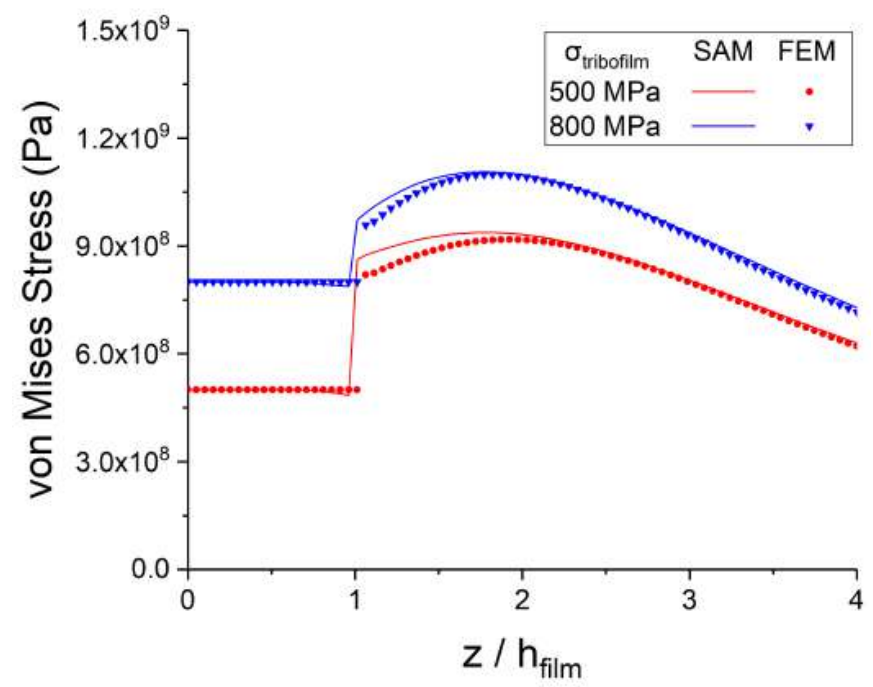

(a)

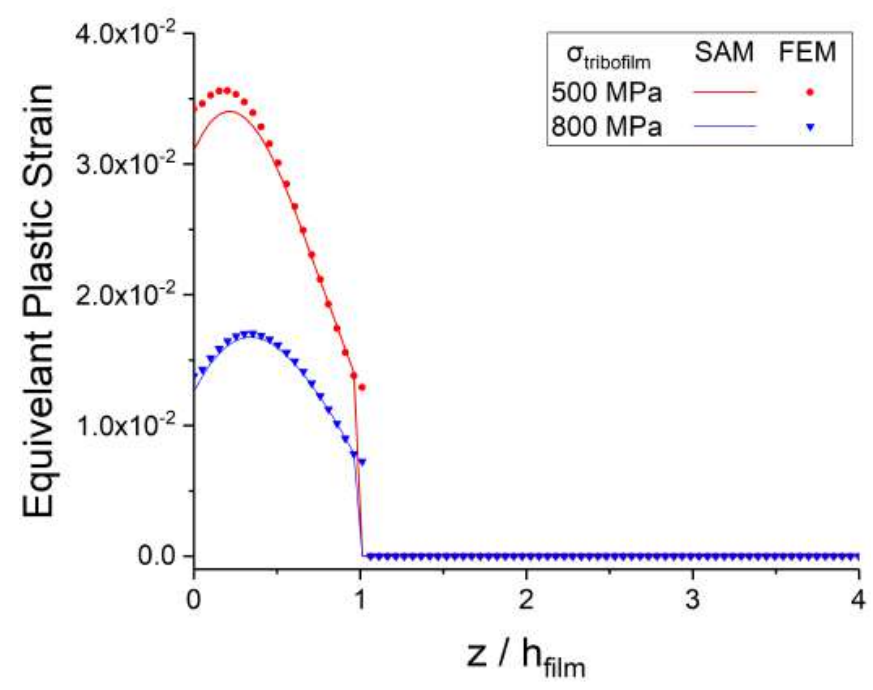

(b)

Fig. 8. Comparison of finite element method (FEM) and semianalytical method (SAM) results along the $z$ axis $(x=0, y=0)$ : (a) von Mises stress; (b) equivalent plastic strain. 
Contour plots of the von Mises stresses and equivalent plastic strain in the $x-z$ plane are shown in Fig. 9 and 10. The results show that plastic strains solely occur in the tribofilm due to its lower yield strength than steel. For the $500 \mathrm{MPa}$ case, the plastic strain tends to concentrate near the bonding interface, while in the $800 \mathrm{MPa}$ case, the plastic strain is more homogeneously distributed.

\section{Mises Stress}
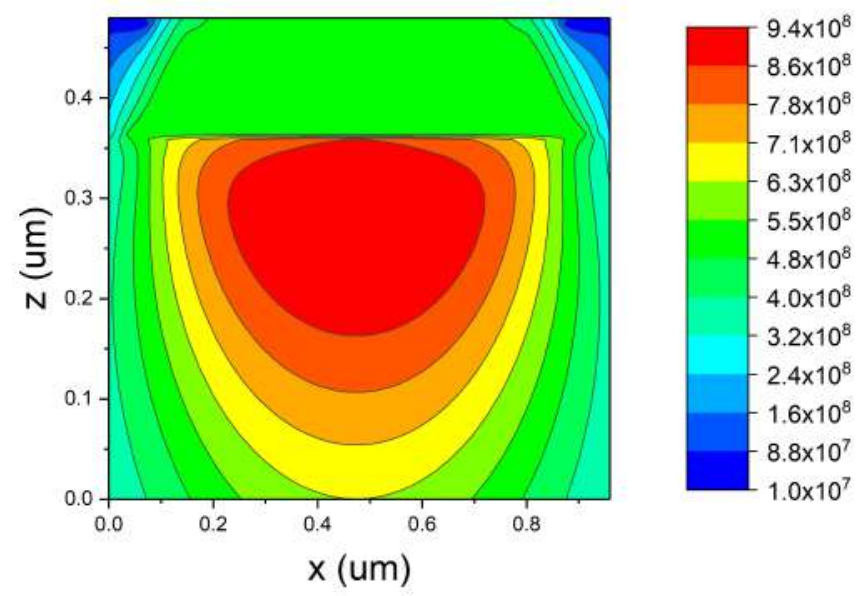

(a)

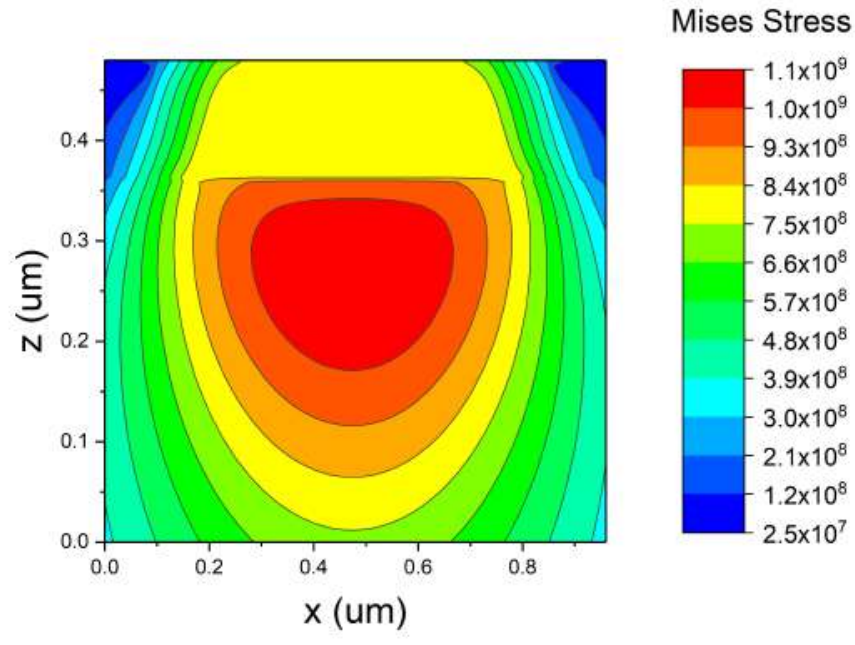

(b)

Fig. 9. Contour of von Mises stress: $(\mathrm{a}) \sigma_{\text {tribofilm }}=500 \mathrm{MPa}$; (b) $\sigma_{\text {tribofilm }}=800 \mathrm{MPa}$. 


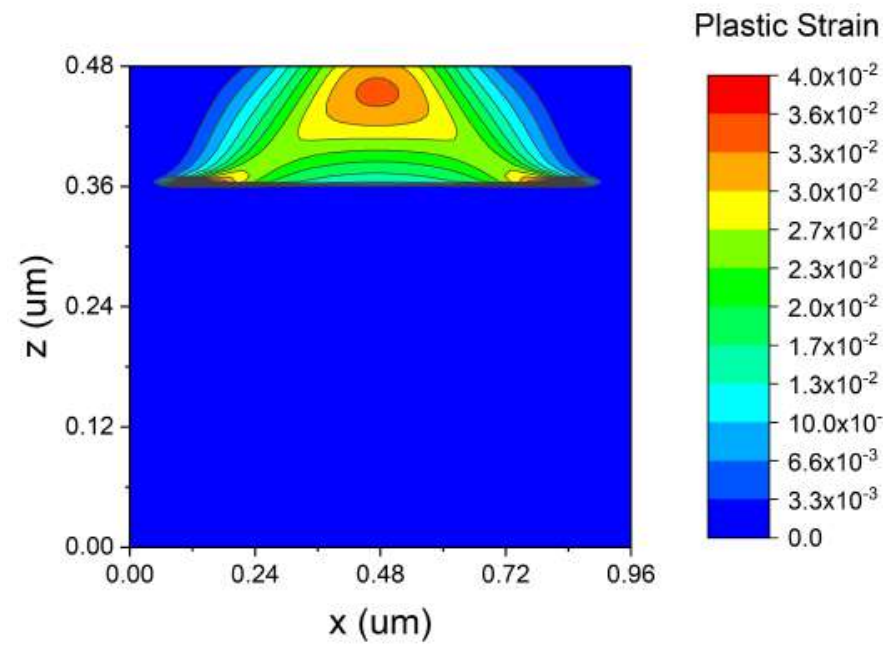

(a)

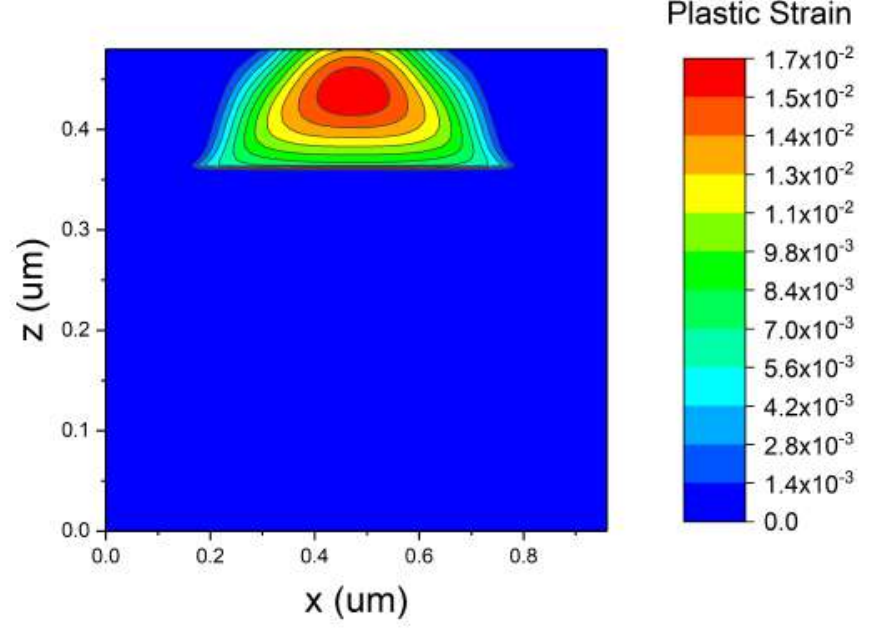

(b)

Fig. 10. Contour of equivalent plastic strain: (a) $\sigma_{\text {tribofilm }}=500 \mathrm{MPa}$; $(\mathrm{b}) \sigma_{\text {tribofilm }}=$ $800 \mathrm{MPa}$.

\subsection{Single Asperity Sliding}

Without considering adhesion effects, the sole mechanism behind friction in single asperity sliding is plastic deformation. The tangential force during the simulated sliding process is shown in Fig. $\mathbf{1 1}$ for both an elastic and an elastoplastic contact. 
In the pure elastic deformation case, the friction force curve acts in a centrosymmetric manner, and thus the net frictional force is zero. However, when plastic deformation is involved, asymmetry of the tangential force can be observed. The reason for this change is that residual displacements modify the surface geometry and break the symmetry of the system. In other words, a certain amount of energy is dissipated during plastic deformation.

Therefore, anything that affects the plastic behavior of the system affects friction. In the following parts, effects of interference depth, tribofilm thickness, elastic/plastic properties of the tribofilm, and the presence of an NC layer on sliding friction are presented and discussed.

Also, the maximum calculated plastic strain in the tribofilm is introduced to measure the risk of tribofilm wear. As indicated in [7] in single asperity sliding, most plastic strain occurs in the tribofilm. The higher the maximum plastic strain is, the more the tribofilm is worn.

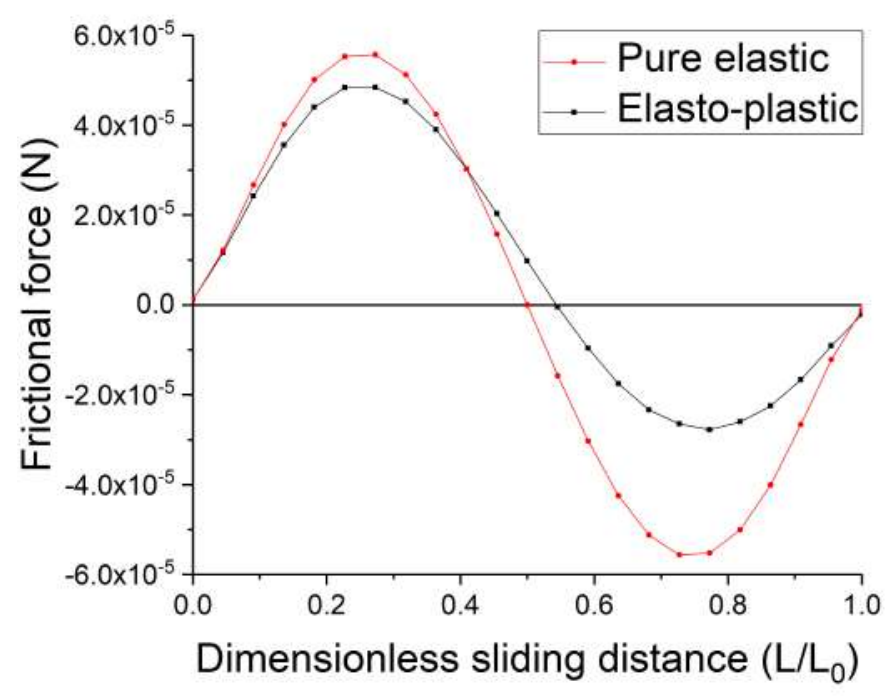

Fig. 11. Tangential force curve for pure elastic and elastoplastic sliding asperity contacts.

\subsubsection{Effects of Interference Depth}


A series of interference depths were applied $(20,35,50$, and $65 \mathrm{~nm})$, while other parameters were held constant (see Tab. 1). The results are shown in Fig. 12, where it becomes clear that the tangential force increases with interference fit, which makes sense as more material is plastically deformed. This is also reflecteds by the increase in calculated coefficient of friction. It should be noted that the maximum plastic strain in the SAM calculations should not exceed $10 \%$ to ensure stable results [37]. The increase in plastic deformation of the tribofilm also indicates that an increase in wear could be expected for the system under consideration, suggesting a relation between friction and wear.

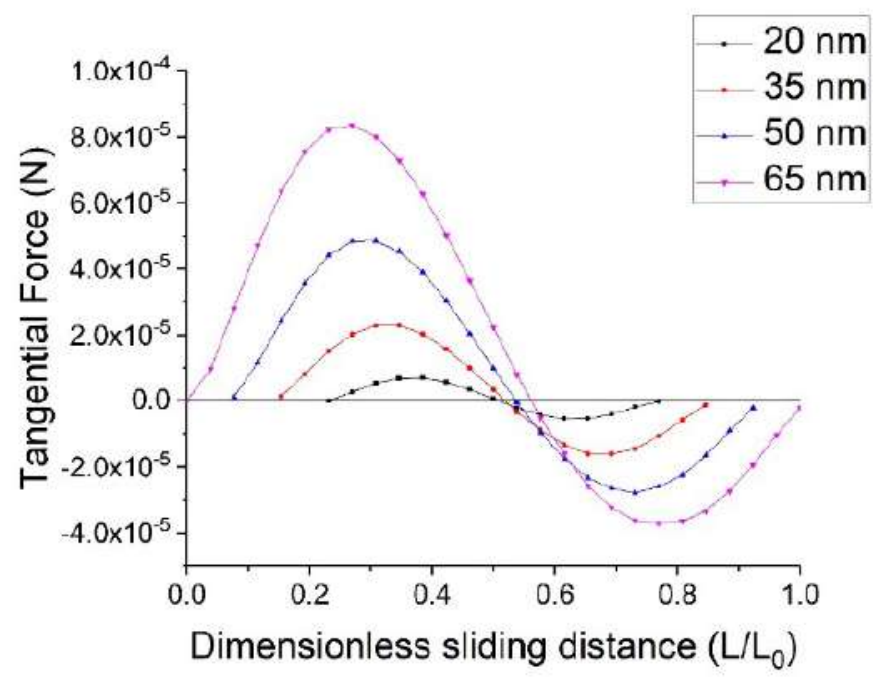

(a) 


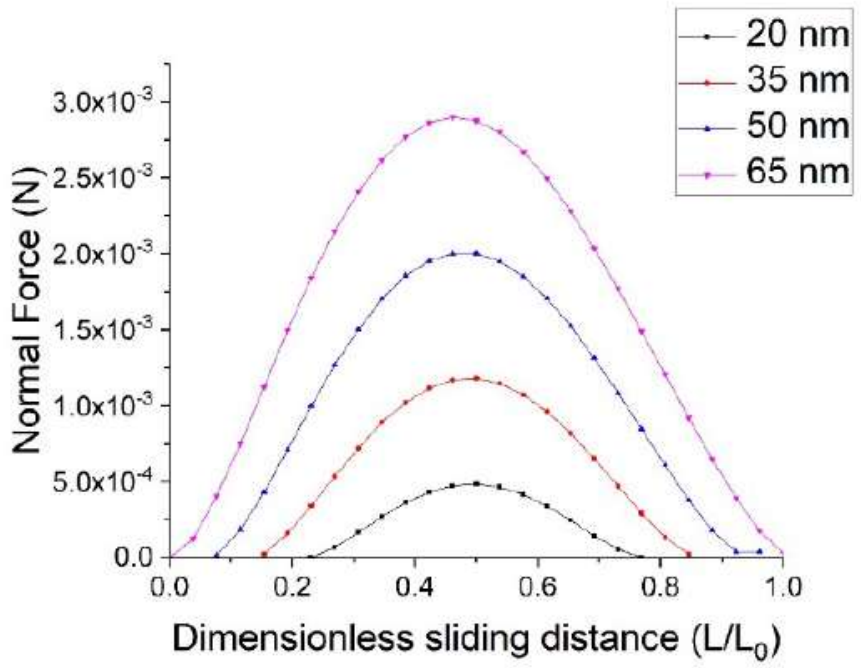

(b)

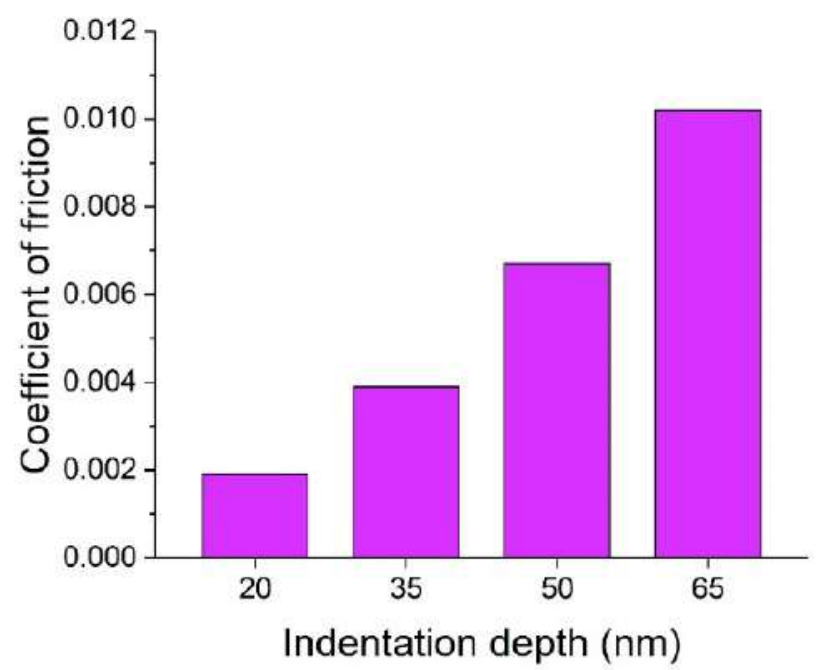

(c) 


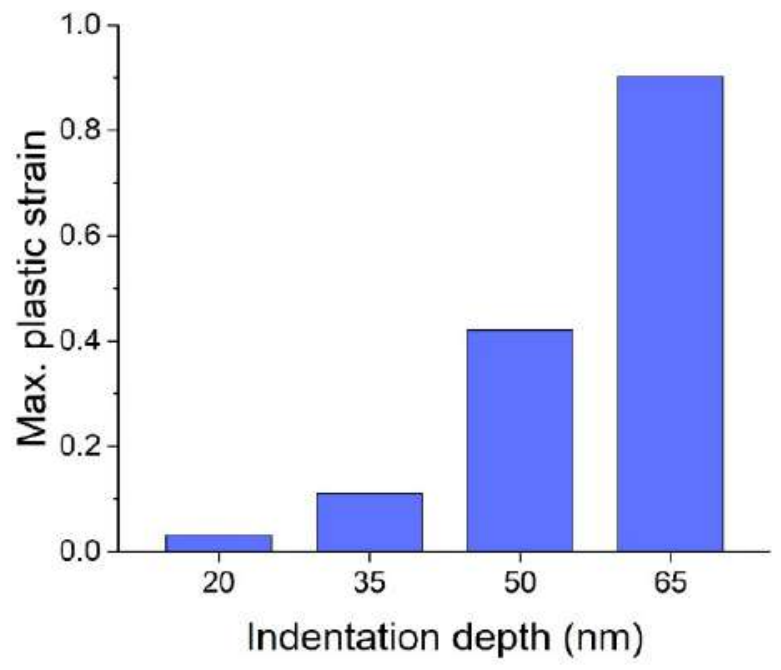

(d)

Fig. 12. Results for different interference depth: (a) frictional force; (b) normal force; (c) coefficient of friction; (d) max. equivalent plastic strain.

\subsubsection{Effects of Tribofilm Thickness}

To investigate the effect of the thickness of the tribofilm, four thicknesses were applied in the model: $10,60,80$, and $100 \mathrm{~nm}$, respectively. The results are shown in Fig. 13. 


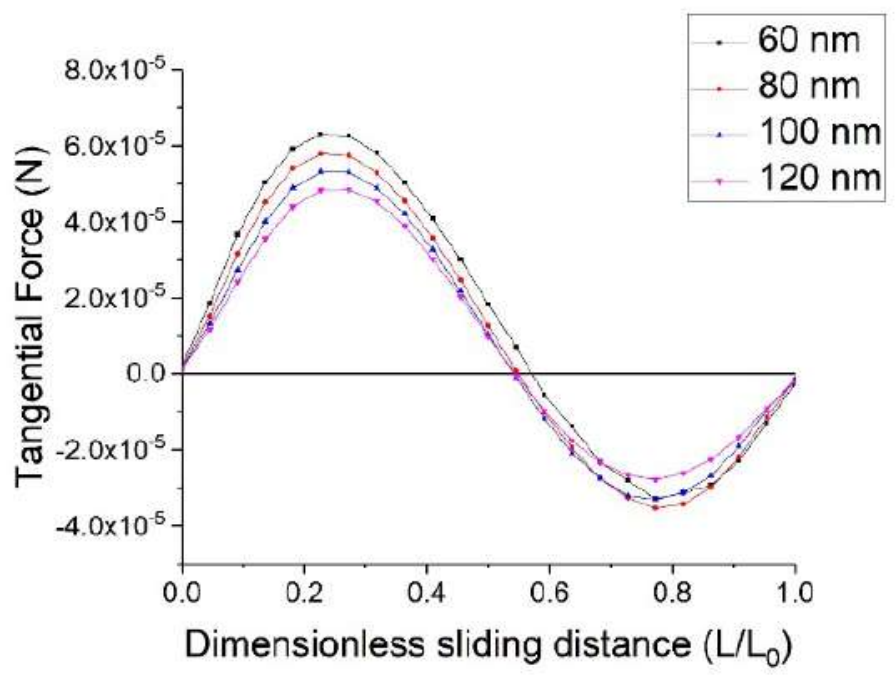

(a)

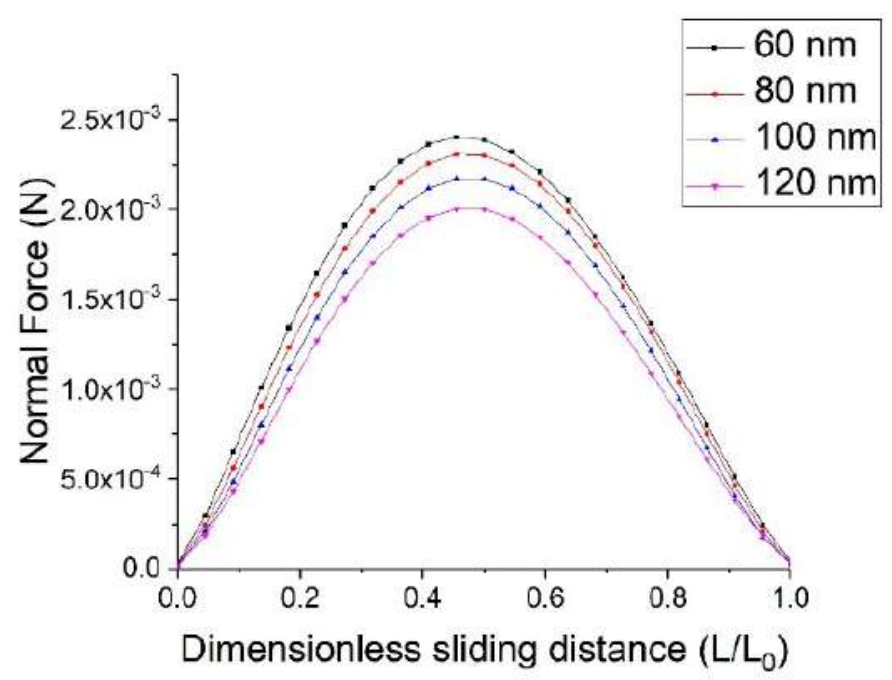

(b) 


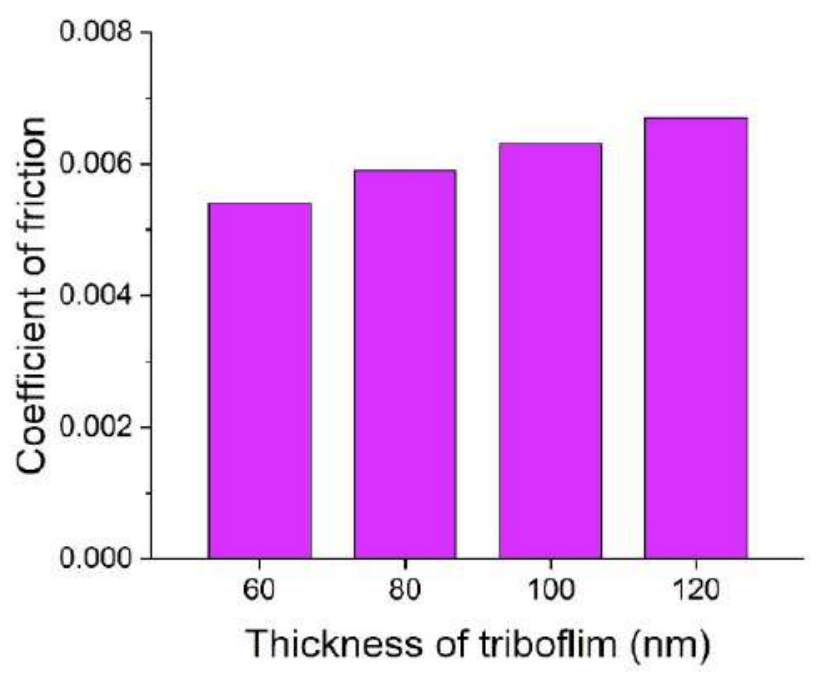

(c)

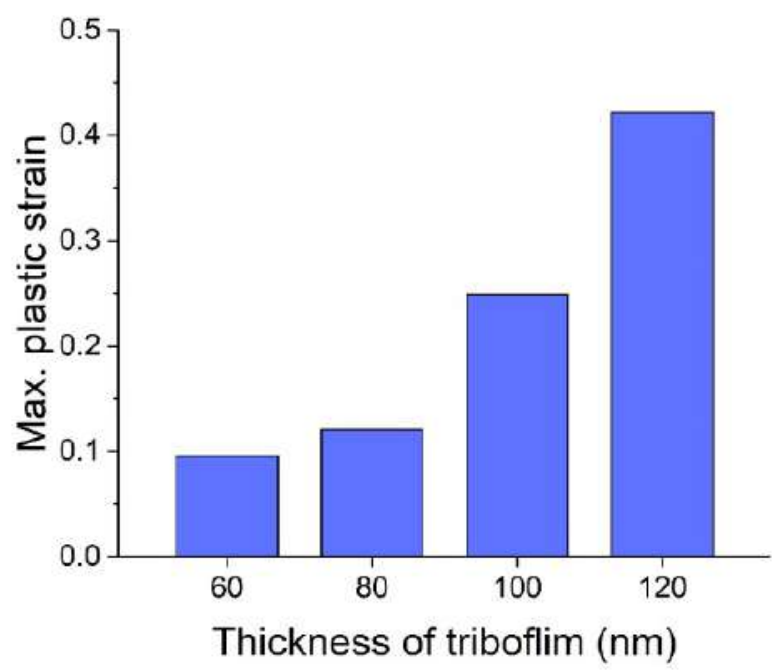

(d)

Fig. 13. Results for different tribofilm thickness: (a) frictional force; (b) normal force; (c) coefficient of friction; (d) max. equivalent plastic strain. 
The normal contact force decreases with thicker tribofilm, while softer tribofilms act like a cushion. The thicker the cushion is, the greater the effect will be. The friction increases with thicker tribofilm, which shows an almost linear relationship. This can be understood in terms of plastic deformation and energy dissipation. However, the relationship between the amount of plasticity and friction is highly nonlinear, showing a greater increase in plasticity than in friction.

\subsubsection{Effects of Elastic Modulus}

The elastic modulus of the tribofilms were chosen from 20 to $65 \mathrm{GPa}$, which refer to tribofilms softer and stiffer than ZDDP (e.g., the reference case, i.e., $35 \mathrm{GPa}$ ).

The results shown in Fig. 14 show that the frictional and normal forces increase with a stiffer tribofilm than expected. Also, a tribofilm with higher elastic modulus leads to a higher coefficient of friction. This is because with a higher elastic modulus, the tribofilm reaches its elastic limit at a lower strain level, which leads to a greater volume of plasticity. Additionally, a higher elastic modulus means more energy is needed for the same amount of plastic deformation. So for these two reasons, more energy is absorbed by the system, thus generating greater friction.

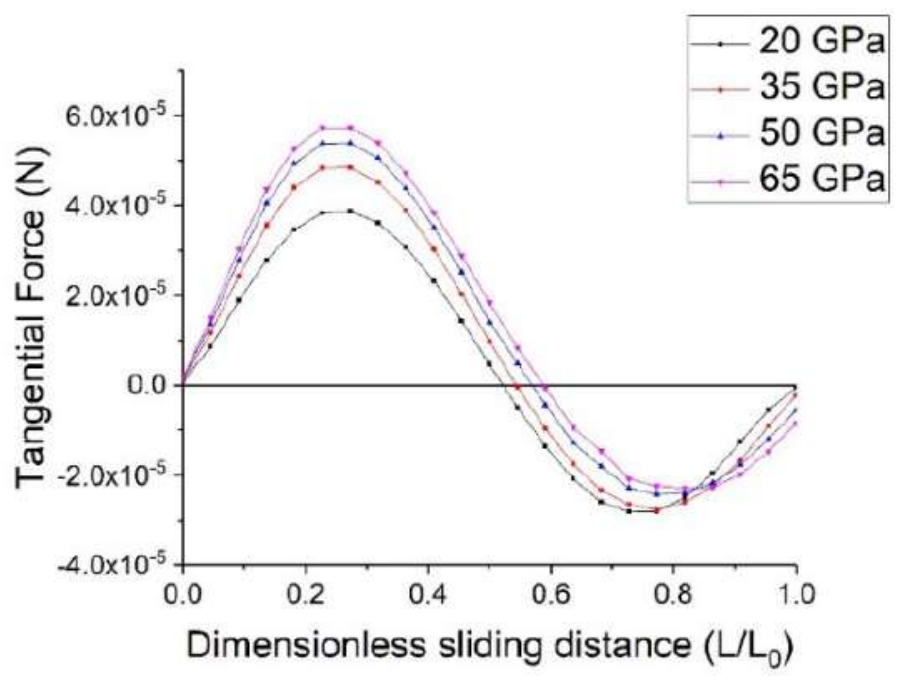

(a) 


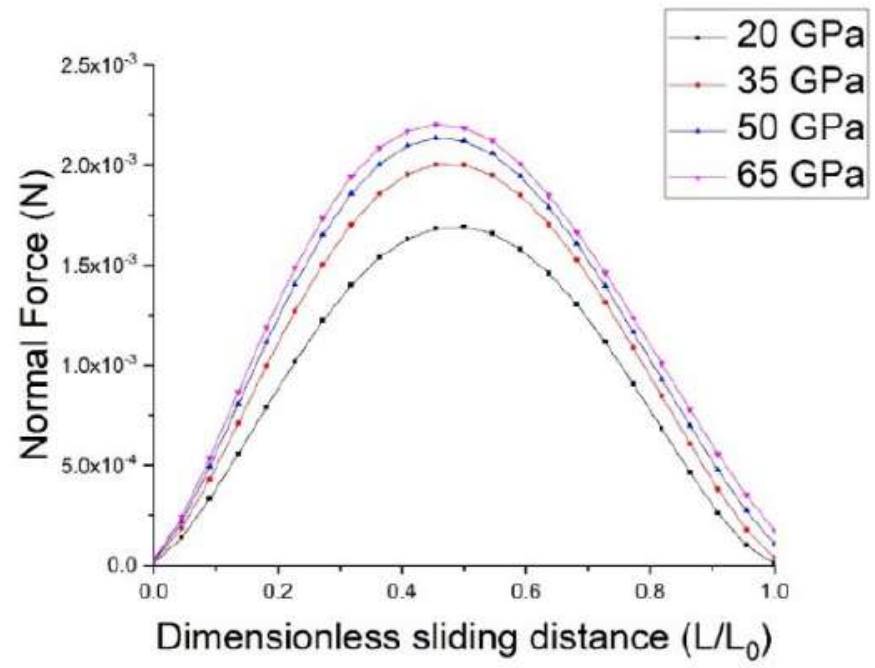

(b)

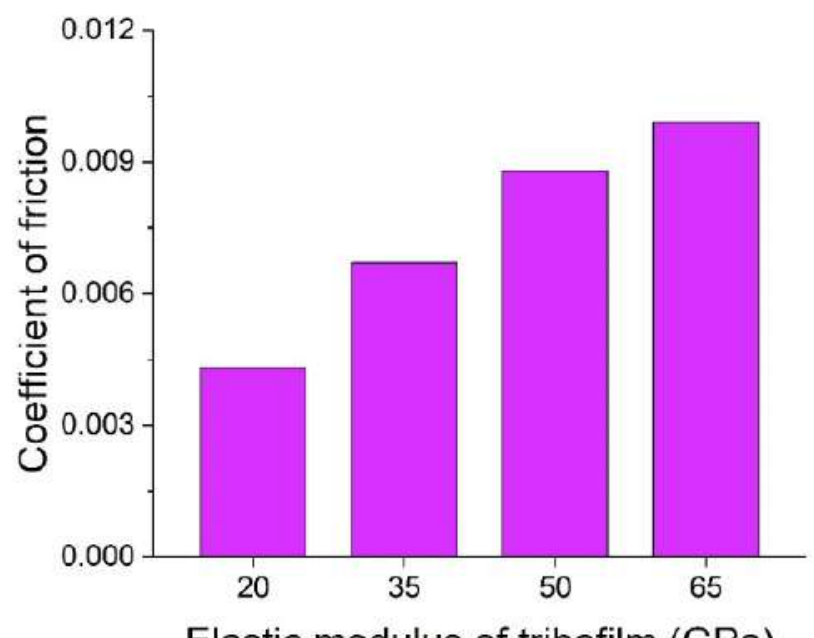

Elastic modulus of tribofilm (GPa)

(c) 


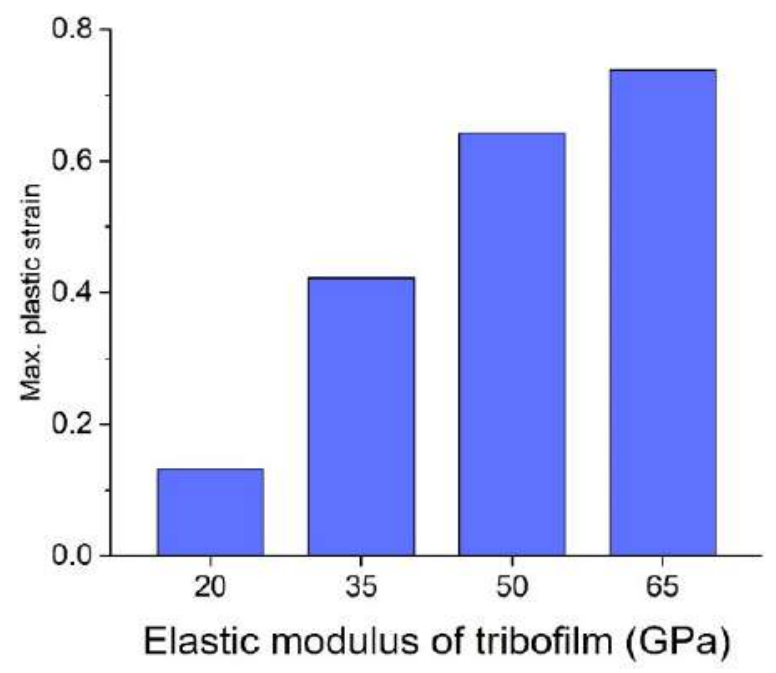

(d)

Fig. 14. Results for different tribofilm elastic modulus: (a) frictional force; (b) normal force; (c) coefficient of friction; (d) max. equivalent plastic strain.

\subsubsection{Effects of Tribofilm Yield Strength}

In this part, the effect of the yield stress of the tribofilm on sliding friction is quantified. The yield stresses of tribofilms were set in a range from 350 to $800 \mathrm{MPa}$, which covers the tribofilms with higher and lower yield strengths than the ZDDP tribofilm.

The results are shown in Fig. 15. 


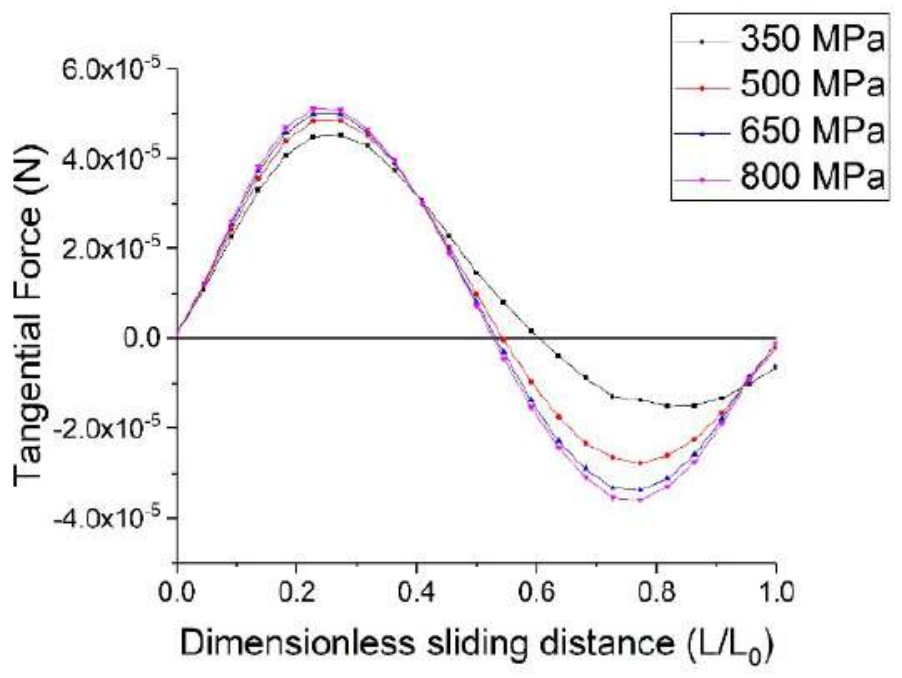

(a)

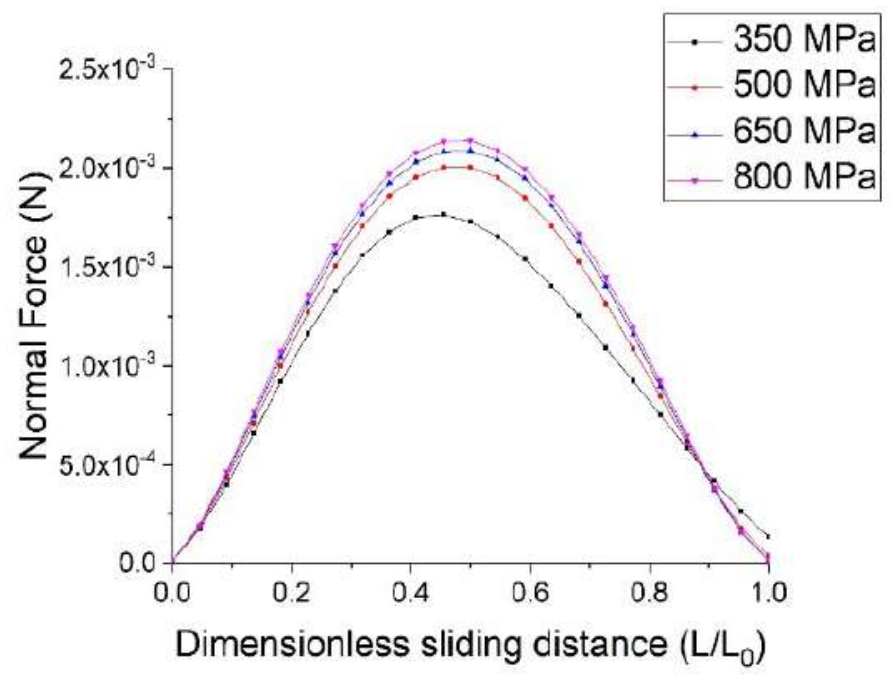

(b) 


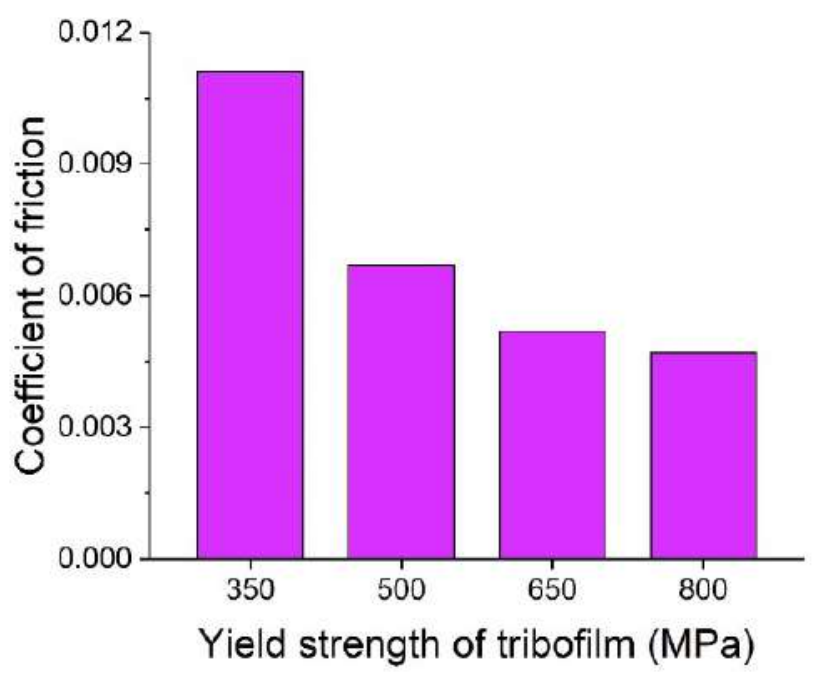

(c)

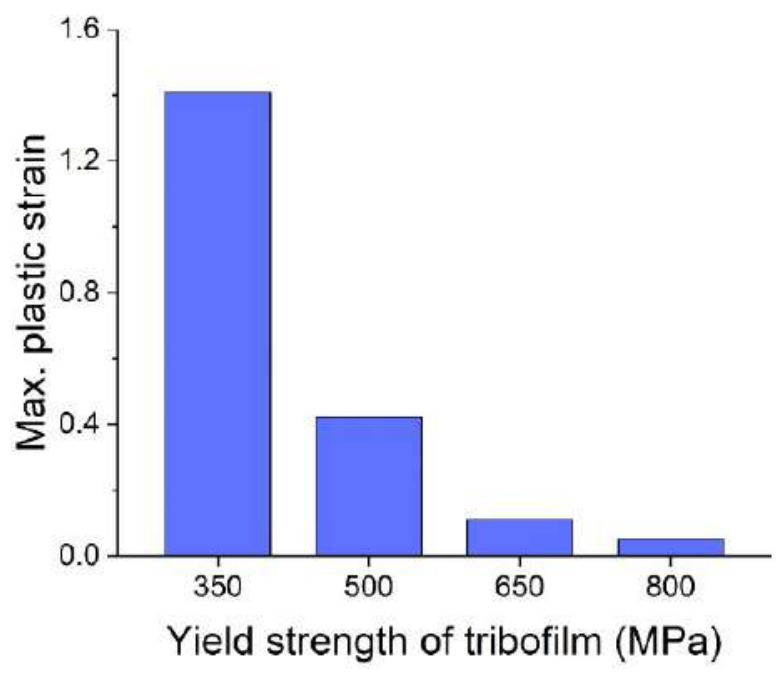

(d)

Fig. 15. Results for different tribofilm yield strengths (a) frictional force; (b) normal force; (c) coefficient of friction; (d) max. equivalent plastic strain. 
From the results, it can be concluded that a tribofilm with a higher yield strength will cause a higher normal force, which is due to less plastic strain/displacement and thus a smaller contact area. Also, the coefficient of friction will decrease due to a higher yield strength of the tribofilm resulting from less plastic deformation. Therefore, the system will be affected less and show lower friction.

\subsubsection{Effects of Presence of the NC Layer}

In this subsection, the effect of the yield stress of the NC layer to sliding friction is quantified. In order to do so, the presence of a nanocrystalline layer is considered. The hardness of the nanocrystalline layer was taken from sample B in [38], see Fig. 16.

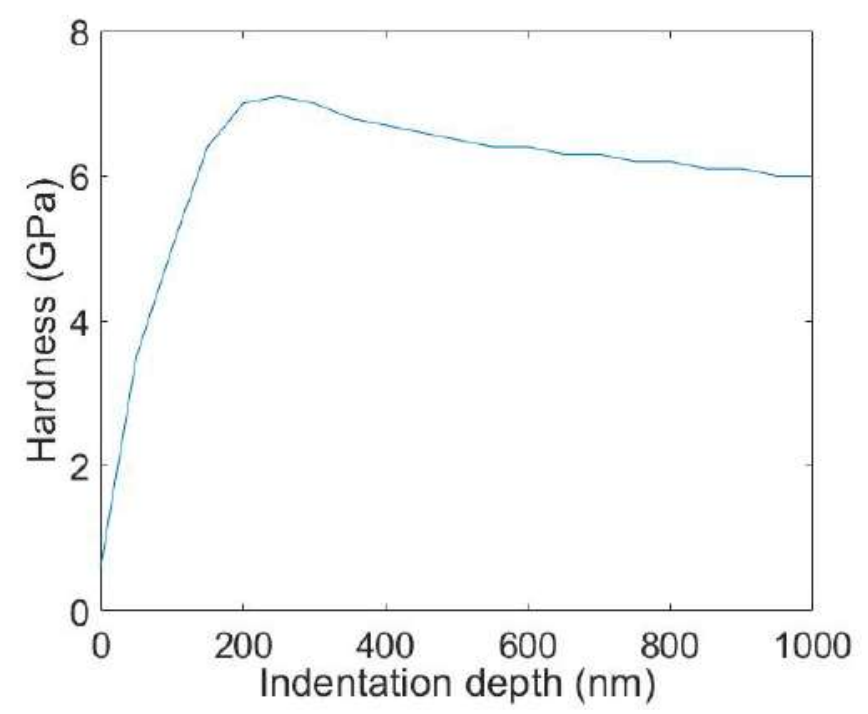

Fig. 16. Hardness of nanocrystalline (NC) layer at different depths, reproduced from Fig. 4 in [38].

The results are shown in Fig. 17. 


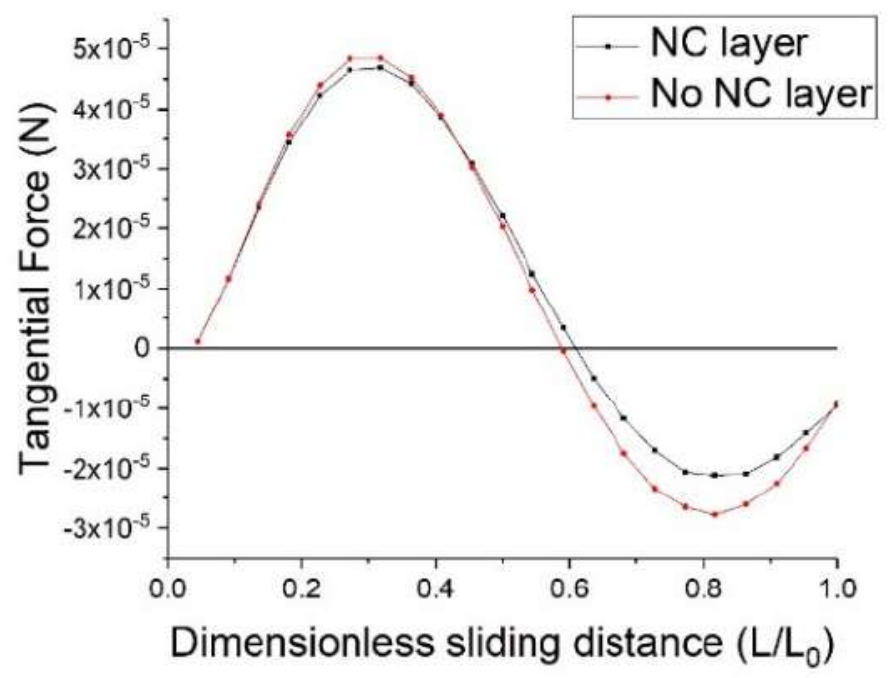

(a)

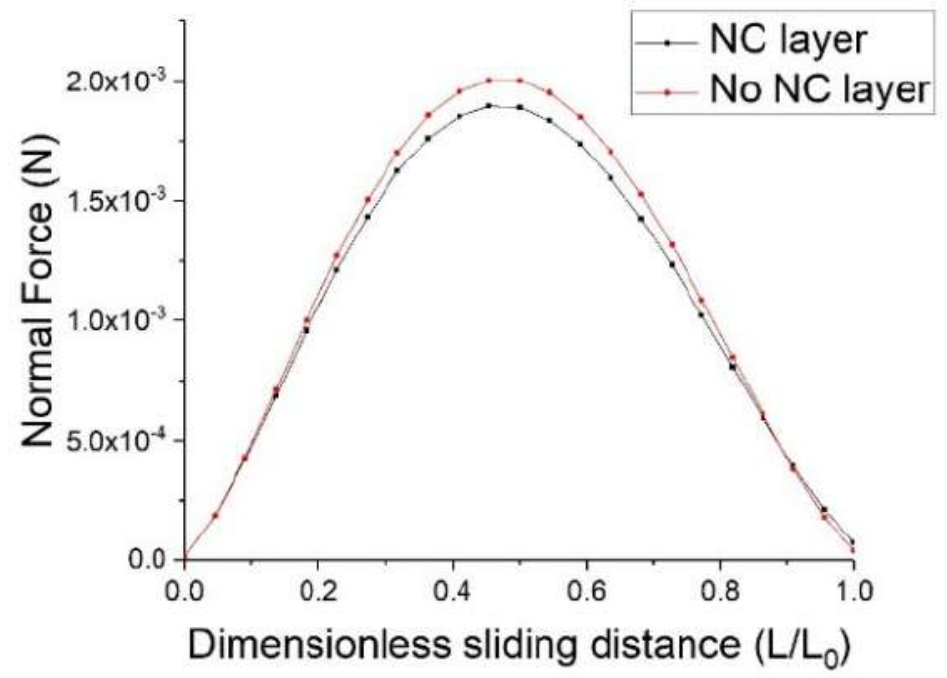

(b) 


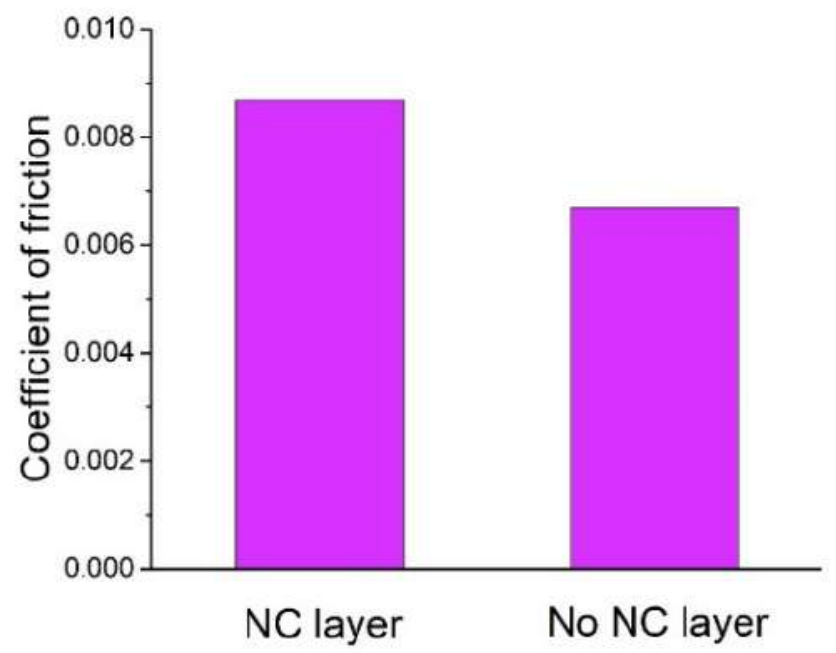

(c)

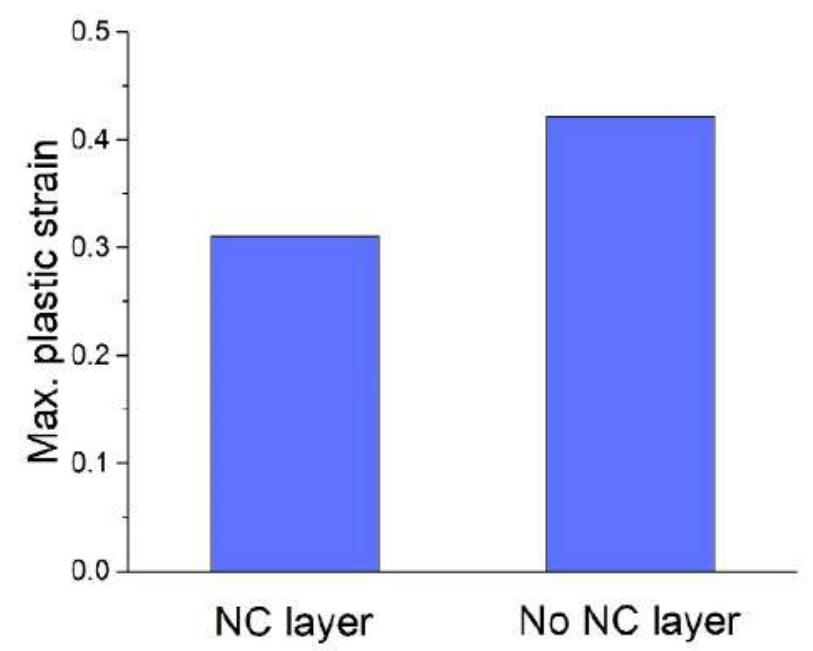

(d)

Fig. 17. Results for presence of NC layer: (a) frictional force; (b) normal force; (c) coefficient of friction; (d) max. equivalent plastic strain. 
With the presence of an NC layer, the frictional and normal contact forces increase. The reason for this effect is similar with a higher yield strength of the tribofilm leading to a higher normal contact force: the less strong NC layer allows higher plastic strain and displacement. Therefore, the geometry can better accommodate the contact deformation.

The coefficient of friction decreases with a higher yield strength of the NC layer. The relatively softer NC layer undergoes higher plastic strain and therefore dissipates more energy, which is consumed as friction. The maximum plastic strains in the tribofilm decrease with the presence of an NC layer because a softer NC layer makes it more possible for the plastic strain to extend downwards into the substrate. This makes the plastic strain accumulate less inside the tribofilm, which reduces the risk of tribofilm damage.

\section{Conclusions}

As in a previous study [7] it was shown that mild wear in the boundary lubrication regime may very well be governed by the plastic deformation of the topmost material. The question is whether friction is also dominated by the mechanism. To answer this question, an elastoplastic coated single asperity sliding model was developed, with surface adhesion being ignored. The sliding process was solved in an incremental manner based on a displacement-driven contact model, accelerated by applying the conjugate gradient method (GCM) and DC-FFT technique. Analyses were carried out at various interference depths and tribofilm parameters, namely, thickness, elastic modulus, and yield strength. The main conclusion is that with adhesion being ignored, to obtain realistic values ( $\sim .1)$ regarding the coefficient of friction, an unrealistically great interference depth would be needed and cause very high plastic strains. This would, however, in practice result in unrealistic values for wear. Thus, in layered boundary lubrication, other sources, e.g., surface adhesion, ploughing, and removal of asperities [39][40] may play a role in sliding friction. It is reported that with asperity failure included, the coefficient of friction is slightly higher than when the asperity failure ignored [12]. These effects, however, are beyond the scope of this paper. Nevertheless, the following insights can be obtained from the results:

(1) The frictional force in a single asperity sliding is closely related to the plastic deformation of the asperities: greater plastic strain, higher sliding friction. 
(2) Interference depth, tribofilm thickness, and the mechanical properties of the tribofilm will all affect the frictional force. Higher interference depth, thicker tribofilm, lower yield strength, and higher elastic modulus will contribute to a higher frictional force.

(3) The presence of a nanocrystalline layer will alter the behavior of the tribosystem. The presence of an NC layer will increase the coefficient of friction and ease the plastic strain accumulating in the tribofilm.

\section{References}

[1] Hardy, W. B., \& Doubleday, I. (1922). Boundary lubrication. The paraffin series. Proceedings of the Royal Society of London. Series A, Containing Papers of a Mathematical and Physical Character, 100(707), 550-574.

[2] Komvopoulos, K., Saka, N., and Suh, N. P. (1985). The mechanism of friction in boundary lubrication. Journal of Tribology, 107(4), 452-462.

[3] Persson, B. N. J. (1993). Theory of friction and boundary lubrication. Physical Review B, 48(24), 18140.

[4] Dupont, P. E., \& Dunlap, E. P. (1993, June). Friction modeling and control in boundary lubrication. In 1993 American Control Conference (pp. 1910-1915). IEEE.

[5] Spikes, H. (2004). The history and mechanisms of ZDDP. Tribology Letters, 17(3), 469-489.

[6] Bosman, R., \& Schipper, D. J. (2011). Mild wear prediction of boundarylubricated contacts. Tribology Letters, 42(2), 169-178.

[7] Bosman, R., \& Schipper, D. J. (2012). Mild wear maps for boundary lubricated contacts. Wear, 280, 54-62.

[8] Stachowiak, G., \& Batchelor, A. W. (2013). Engineering tribology. ButterworthHeinemann.

[9] Liu, G., Zhu, J., Yu, L., \& Wang, Q. J. (2001). Elasto-plastic contact of rough surfaces. Tribology Transactions, 44(3), 437-443.

[10] Kogut, L., \& Etsion, I. (2002). Elastic-plastic contact analysis of a sphere and a rigid flat. Journal of Applied Mechanics, 69(5), 657-662.

[11] Jackson, R. L., Duvvuru, R. S., Meghani, H., \& Mahajan, M. (2007). An analysis of elasto-plastic sliding spherical asperity interaction. Wear, 262(1-2), 210-219. 
[12] Mulvihill, D. M., Kartal, M. E., Nowell, D., \& Hills, D. A. (2011). An elasticplastic asperity interaction model for sliding friction. Tribology International, 44(12), 1679-1694.

[13] Cheng, S., Luan, B., \& Robbins, M. O. (2010). Contact and friction of nanoasperities: effects of adsorbed monolayers. Physical Review E, 81(1), 016102.

[14] Yang, C., \& Persson, B. N. J. (2008). Molecular dynamics study of contact mechanics: contact area and interfacial separation from small to full contact. Physical Review Letters, 100(2), 024303.

[15] Anciaux, G., \& Molinari, J. F. (2009). Contact mechanics at the nanoscale, a 3D multiscale approach. International Journal for Numerical Methods in Engineering, 79(9), 1041-1067.

[16] Dubujet, P., Ghaoudi, A., Chaze, M., \& Sidoroff, F. (1995, September). Particulate and Granular Simulation of Third Body Behavior. In 22nd LeedsLyon Symposium on Tribology (Vol. 31, pp. 355-365). Elsevier.

[17] Iordanoff, I., Seve, B., \& Berthier, Y. (2002). Solid third body analysis using a discrete approach: influence of adhesion and particle size on macroscopic properties. Journal of Tribology, 124(3), 530-538.

[18] Tan, Y., Yang, D., \& Sheng, Y. (2009). Discrete element method (DEM) modeling of fracture and damage in the machining process of polycrystalline $\mathrm{SiC}$. Journal of the European Ceramic sSociety, 29(6), 1029-1037.

[19] Fillot, N., lordanoff, I., \& Berthier, Y. (2007). Modelling third body flows with a discrete element method-a tool for understanding wear with adhesive particles. Tribology International, 40(6), 973-981.

[20] Müller, M., Ostermeyer, G. P., \& Bubser, F. (2013). A contribution to the modeling of tribological processes under starved lubrication. Tribology International, 64, 135-147.

[21] Müller, M., \& Ostermeyer, G. P. (2007). A cellular automaton model to describe the three-dimensional friction and wear mechanism of brake systems. Wear, 263(7-12), 1175-1188.

[22] Dmitriev, A. I., Österle, W., Wetzel, B., \& Zhang, G. (2015). Mesoscale modeling of the mechanical and tribological behavior of a polymer matrix composite based on epoxy and 6 vol.\% silica nanoparticles. Computational Materials Science, 110, 204-214.

[23] Renouf, M., Massi, F., Fillot, N., \& Saulot, A. (2011). Numerical tribology of a dry contact. Tribology International, 44(7-8), 834-844.

[24] Ju, Y., and Farris, T. N. (1996). Spectral analysis of two-dimensional contact problems. Journal of Tribology, 118(2), 320-328. 
[25] Polonsky, I. A., \& Keer, L. M. (1999). A numerical method for solving rough contact problems based on the multi-level multi-summation and conjugate gradient techniques. Wear, 231(2), 206-219.

[26] Liu, S., Wang, Q., \& Liu, G. (2000). A versatile method of discrete convolution and FFT (DC-FFT) for contact analyses. Wear, 243(1-2), 101-111.

[27] Jacq, C., Ne' lias, D., Lormand, G., \& Girodin, D. (2002). Development of a three-dimensional semi-analytical elastic-plastic contact code. Journal of Tribology, 124(4), 653-667.

[28] Chiu, Y. P. (1977). On the stress field due to initial strains in a cuboid surrounded by an infinite elastic space. Journal of Tribology, 44(4), 587-590.

[29] Chiu, Y. P. (1978). "On the stress field and surface deformation in a half space with a cuboidal zone in which initial strains are uniform. Journal of Tribology, 45(2), 302-306.

[30] Wang, Z., Yu, H., \& Wang, Q. (2016). Analytical solutions for elastic fields caused by eigenstrains in two joined and perfectly bonded half-spaces and related problems. International Journal of Plasticity, 76, 1-28.

[31] Boucly, V., Nélias D., \& Green, I. (2007). Modeling of the rolling and sliding contact between two asperities. Journal of Tribology, 129(2), 235-245.

[32] Simo, J. C., \& Taylor, R. L. (1985). Consistent tangent operators for rateindependent elastoplasticity. Computer Methods in Applied Mechanics and Engineering, 48(1), 101-118.

[33] Green, A. P. (1955). Friction between unlubricated metals: a theoretical analysis of the junction model. Proceedings of the Royal Society of London. Series A. Mathematical and Physical Sciences, 228(1173), 191-204.

[34] King, R. B., \& O'sullivan, T. C. (1987). Sliding contact stresses in a twodimensional layered elastic half-space. International Journal of Solids and Structures, 23(5), 581-597.

[35] Aktary, M., McDermott, M. T., \& McAlpine, G. A. (2002). Morphology and nanomechanical properties of ZDDP antiwear films as a function of tribological contact time. Tribology Letters, 12(3), 155-162.

[36] van der Heide, E., \& Schipper, D. J. (2004). On the frictional heating in single summit contacts: towards failure at asperity level in lubricated systems. Journal of Tribology, 126(2), 275-280.

[37] Bosman, R. (2011). Mild wear modeling in the boundary lubrication regime. University of Twente.

[38] Shakhvorostov, D., Pöhlmann, K., \& Scherge, M. (2006). Structure and mechanical properties of tribologically induced nanolayers. Wear, 260(4-5), 433-437. 
[39] Yang, L., Wang, D., Guo, Y., \& Liu, S. (2016). Tribological behaviors of quartz sand particles for hydraulic fracturing. Tribology International, 102, 485-496.

[40] Sandeep, C. S., \& Senetakis, K. (2018). Grain-scale mechanics of quartz sand under normal and tangential loading. Tribology International, 117, 261-271. 



\section{Paper B}

Wang, C., \& Schipper, D. J. (2020). A numerical-analytical approach to determining the real contact area of rough surface contact. TribologyMaterials, Surfaces \& Interfaces, 1-11. 



\title{
A numerical-analytical approach to determining the real contact area of rough surface contact
}

\author{
Can Wang * and Dik J. Schipper \\ Laboratory for Surface Technology and Tribology, Department of Engineering Technology, \\ University of Twente, 7500 AE Enschede, The Netherlands \\ *Correspondence: c.wang-1@utwente.nl
}

\begin{abstract}
The objective of this study is to improve the accuracy of real contact area calculated by the semi-analytical method (SAM). Two types of surface pairs are investigated: an analytically generated sinusoidal wavy surface against a rigid flat, and a pair of real rough surfaces. The results suggest that the real contact area calculated by the SAM is extremely sensitive to the resolution of input, i.e. the grid size. The SAM results of the real contact areas show poor convergence, especially in the case of the real rough surfaces. The main reason for this difference is the "over-covering" effect when SAM calculates the real contact area. An exponential extrapolation technique is proposed to predict the real contact area values when further refinement of the grid resolution is unfeasible.
\end{abstract}

Keywords: Real contact area; Semi-analytical method; Grid sensitivity; Extrapolation

\section{Introduction}

Contact between rough surfaces has long been a matter of concern in tribology. Accurate characterization of the contact pressure and the real contact area in rough surface contacts are desired because of their linkage to the tribological performances. Both contact pressures and real contact areas are considered essential factors of dry friction mechanisms [1]. Further, stresses generated by contact pressures are crucial for common wear mechanisms including abrasive and fatigue wear [2]. Therefore, obtaining information of real contact areas and contact pressures will contribute to the theoretical estimates of friction and wear. While "insitu" measurements of contact pressure and real contact area by experiments are 
still difficult [3], numerical simulations are extensively employed to perform a rough surface contact analysis, thanks to their flexibility and versatility.

The semi-analytical method (SAM) provides an effective approach in numerically solving contacts of rough surfaces. With the acceleration techniques of fast Fourier transform (DC-FFT) and the conjugate gradient method [1], SAM is applied to solve various rough surface contact problems efficiently by feeding in different analytical solutions. With Love's equation [5], the simple homogeneous elastic contacts were solved by Bjorklund and Andersson [6]. By applying O'Sullivan and King's solution of layered structure [7], Nogi and Kato [8] solved the elastic single layered contact, Cai and Bhushan [9] solved the elastic bi-layered contact, and Yu et. al [10] further solved the elastic contact of materials with arbitrary number of layers. Jacq et. al. [11] extended the SAM to solving elasto-plastic contact with the help of Chiu's analytical solution of eigenstrains [12][13]. With the same analytical solutions, a series of contact between materials with inclusions were also considered [14][15][16]. Despite the versatility in dealing with variety of contact types, SAM is also efficient in computational time. As opposed to the finite element method, which needs a very fine mesh and a large calculating domain, SAM requires the computation to be made only on the contact domain involved [1]. Due to these advantages, SAM is extensively employed in a variety of cases to understand the complicated tribological phenomena including running-in [17][18][19], fretting [20][21][22] and partial-slip [23][24][25] based on the rough surface contact calculation.

As a numerical method, SAM requires discretized surface geometry data as input. However, a dilemma is encountered when choosing the resolution of input data to model rough surface contacts. On one hand, the resolution of the surface inputs should be high enough to accurately model the real rough surface contact. It has long been known that material surfaces consist of molecular and super-molecular structures. Sizes of asperities can be as small as nanoscale ( $10 \mathrm{~nm})$. Under certain circumstances, the local real contact area can be extremely small when single asperity contacts are formed. In such cases, high resolution of the surface inputs should be applied so that these small asperities can be accurately depicted. However, very high resolution of input is not always feasible due to the computational costs of SAM algorithms. For a simple elastic contact calculation, the complexity of the SAM algorithm is $\mathrm{O}\left(\mathrm{N}(\log N)^{2}\right)$ and the memory consumption is $\mathrm{O}\left(\mathrm{N}^{2}\right)[4]$, suggesting that doubling the input resolutions in both $\mathrm{x}$ and $\mathrm{y}$ directions means 8 times more calculating time and 4 times more memory demands. For some elasto-plastic contact cases, the complexity will be even dramatically higher because the calculation 
domains are 3D instead of 2D for the simple elastic contact cases. Wang et al. reported an $864 \mathrm{MNL} * \ln (8 \mathrm{MNL})+432 \mathrm{MNL} * \ln (4 \mathrm{MN})$ complexity for an algorithm solving an arbitrarily shaped inclusion in an elasto-plastic contact [26]. Therefore, compromises on input resolutions sometimes have to be made in order that calculations can be completed within a reasonable time duration.

Due to this conflict, the effects of input surface resolution on the accuracy of SAM results need to be examined carefully. A sensitivity study is therefore necessary. In this article, the sensitivity on the maximum contact pressure and real contact area results with respect to the resolution of surface inputs are studied. First, the contact between an analytically generated wavy surface and a rigid flat are studied and compared with the analytical solution. The investigation is then extended to a real surface contact by studying the contacts of a steel ball and a grinded flat before and after running in.

\section{Theory and methods}

In this section, a SAM model for the rough surface contact with normal load is presented, allowing the pressure distribution and real contact area to be calculated.

First, a reference coordinate system is introduced, as shown in Fig. 1. For simplicity but without loss of generality, a contact of an elastic object loading on a rigid flat is considered,

where:

F is the normal load applied

$p$ is the contact pressure distribution due to the load

$\mathrm{u}$ is the surface displacement due to contact pressure

$h$ is the initial surface separation relative to the initial undeformed geometry

$\delta_{z}$ is the rigid body movement. 


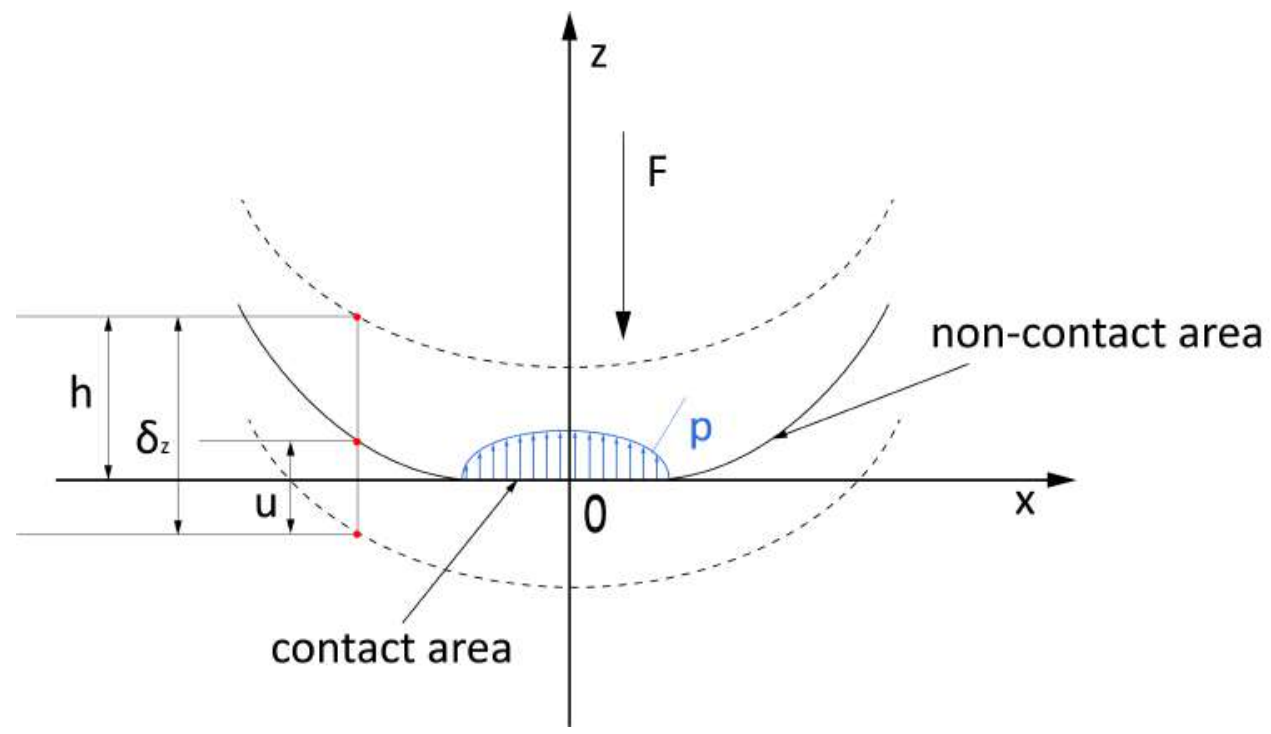

Fig. 1. Schematic of the contact.

In the whole domain in which contact and noncontact areas both exist the following Kuhn-Tucker complementary conditions hold:

$$
u+h=\delta_{z} \quad \text { where } p>0
$$

and

$$
u+h>\delta_{z} \quad \text { where } p=0
$$

Eq. (1) indicates that in the contact area surface separation must be zero (no penetration). In the noncontact areas, however, surface separation should be greater than zero, which is shown in Eq. (2).

To solve this problem numerically by the semi-analytical method, the whole domain, including both the contact and non-contact area, is firstly discretized into $\mathrm{m}^{*} \mathrm{n}$ grids, where each grid has a size of $2 a * 2 b$ (Fig. 2). 


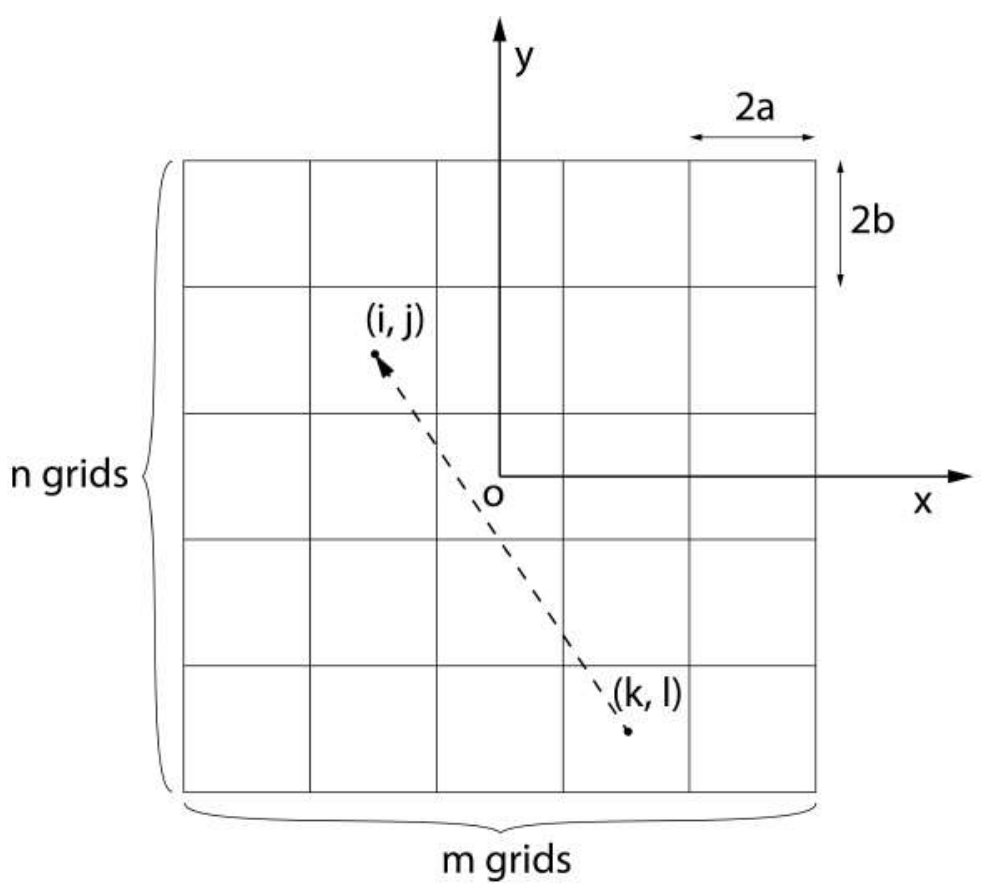

Fig. 2. Schematic of domain discretization.

The equation can be further re-written in discretized manner:

$\forall(\boldsymbol{i}, \boldsymbol{j}) \in \boldsymbol{I}$

$$
u_{i j}+h_{i j}=\delta_{z} \quad(i, j) \in I_{c}
$$

and

$$
u_{i j}+h_{i j}>\delta_{z} \quad(i, j) \notin I_{c}
$$

Where $\boldsymbol{I}$ represents the whole calculating domain and $\boldsymbol{I}_{\boldsymbol{c}}$ represents the real contact area where $\boldsymbol{p}_{\boldsymbol{i} \boldsymbol{j}}>\mathbf{0}$.

In order to solve the pressure distribution $\boldsymbol{p}_{\boldsymbol{i} \boldsymbol{j}}$, it is necessary to link $\boldsymbol{u}_{\boldsymbol{i} \boldsymbol{j}}$ to $\boldsymbol{p}_{\boldsymbol{i} \boldsymbol{j}}$. Here the analytical solution of Love for normal deflections under a uniform normal pressure [5] is introduced: 


$$
\boldsymbol{u}_{i j, k l}=\boldsymbol{K}_{i-j, k-l} * \boldsymbol{p}_{k l}
$$

where $\boldsymbol{u}_{\boldsymbol{i} \boldsymbol{j}, \boldsymbol{k} \boldsymbol{l}}$ is the deflection in grid $(\boldsymbol{i}, \boldsymbol{j})$ caused by the contact pressure in grid $(\boldsymbol{k}, \boldsymbol{l})$, and the coefficient $\boldsymbol{K}_{\boldsymbol{i}-\boldsymbol{j}, \boldsymbol{k}-\boldsymbol{l}}$ written as:

$K_{i-j, k-l}=\frac{1-v}{2 \pi G} *\left(\begin{array}{c}(x+a) * \ln \frac{y+b+\sqrt{(y+b)^{2}+(x+a)^{2}}}{y-b+\sqrt{(y-b)^{2}+(x+a)^{2}}}+(y+b) * \ln \frac{x+a+\sqrt{(y+b)^{2}+(x+a)^{2}}}{x-a+\sqrt{(y+b)^{2}+(x-a)^{2}}} \\ \left.\left.+(x-a) * \ln \frac{y-b+\sqrt{(y-b)^{2}+(x-a)^{2}}}{y+b+\sqrt{(y+b)^{2}+(x-a)^{2}}}\right)+(y-b) * \ln \frac{x-a+\sqrt{(y-b)^{2}+(x-a)^{2}}}{x+a+\sqrt{(y-b)^{2}+(x+a)^{2}}}\right)\end{array}\right)(6)$ $(x, y$ are the horizontal and vertical distance from $(k, l)$ to $(i, j)$, see Fig. 2$)$

The total deflection $\boldsymbol{u}_{\boldsymbol{i} \boldsymbol{j}}$ is the sum of all the contact pressure contributions in the calculating domain. Now Eq. (3) and (4) can be written as follows

$$
\begin{aligned}
& \sum_{(i, j) \in I_{c}} K_{i-j, k-l} p_{k l}+h_{i j}=\delta_{z} \\
& \sum_{(i, j) \notin I_{c}} K_{i-j, k-l} p_{k l}+h_{i j}>\delta_{z}
\end{aligned}
$$

This is a set of linear equations with the pressure distribution being explicitly included as unknowns, which can be efficiently solved by the conjugate gradient method proposed in literature [1].

First, a guess pressure distribution is made, and then the following iteration steps from (1) to (5) will be performed:

(1) update the gap 


$$
\begin{gathered}
g_{i j}=-\sum_{(i, j) \in I} K_{i-j, k-l} p_{k, l}-h_{i j} \\
\bar{g}=\frac{1}{N_{c}} \sum_{(k, l) \in I_{c}} g_{k l} \\
g_{i j}=g_{i j}-\bar{g} \quad(i, j) \in I
\end{gathered}
$$

(2) calculate the new conjugate direction

$$
\begin{gathered}
G=\sum_{(k, l) \in I_{c}} g_{i j}{ }^{2} \\
t_{i j}=g_{i j}+\delta\left(\frac{G}{G_{\text {old }}}\right) t_{i j} \quad(i, j) \in I_{c} \\
t_{i j}=0 \quad(i, j) \notin I_{c}
\end{gathered}
$$

(3) calculate the new descending step length

$$
\begin{gathered}
r_{i j}=\sum_{(k, l) \in \Gamma} K_{i-j, k-l} t_{k l} \quad(i, j) \in I \\
\bar{r}=\frac{1}{N_{c}} \sum_{(k, l) \in I_{c}} r_{k l}
\end{gathered}
$$




$$
r_{i j}=r_{i j}-\bar{r} \quad(i, j) \in I
$$

$$
\tau=\frac{\sum_{(k, l) \in I_{c}} g_{i j} t_{i j}}{\sum_{(k, l) \in I_{c}} r_{i j} t_{i j}}
$$

(4) update the pressure distribution, enforce the inequalities and force balance

$$
\begin{aligned}
& p_{i j}=p_{i j}-\tau * t_{i j} \quad(i, j) \in I_{c} \\
& I_{o l}=\left\{(i, j) \in I: \quad p_{i j}=0, g_{i j}<0\right\} \\
& p_{i j}=p_{i j}-\tau * g_{i j} \quad(i, j) \in I_{o l} \\
& \boldsymbol{F}^{\prime}=\boldsymbol{a} * \boldsymbol{b} * \sum_{(k, l) \in I} \boldsymbol{p}_{i j} \\
& \boldsymbol{p}_{\boldsymbol{i} j}=\left(\frac{\boldsymbol{F}^{\prime}}{\boldsymbol{F}}\right) * \boldsymbol{p}_{\boldsymbol{i j}}
\end{aligned}
$$

(5) check the relative error

$$
=\boldsymbol{a} * \boldsymbol{b} * \sum_{(i, j) \in I}\left|p_{i j}-p_{i j}^{o l d}\right|
$$


If $\varepsilon>\varepsilon_{0}$, where $\varepsilon_{\mathbf{0}}$ is the preset tolerance, another iteration will be performed starting from step (1). Otherwise, the iteration stops and the current $\boldsymbol{p}_{\boldsymbol{i j}}$ results are the pressure distribution desired. The real contact area can also be acquired by counting the area where $\boldsymbol{p}_{\boldsymbol{i j}}>\mathbf{0}$.

\section{Results and discussions}

\subsection{Wavy surfaces}

In this part, the analysis is conducted on the contact between an elastic sinusoidal wavy surface and a rigid flat, with different nominal contact pressures. The maximum contact pressure and real contact area are calculated both analytically and by the SAM model with different resolutions of input. The results are compared and the errors of the SAM results as a function of the resolutions of input are investigated and discussed.

An elastic sinusoidal waviness was considered, see Fig. 3. The geometric shape of the sinusoidal wave is defined by Eq. (25), which is also illustrated in literature [27]:

$$
z(x, y)=\Delta_{1} \cos \left(\frac{2 \pi x}{\lambda_{1}}\right)+\Delta_{2} \cos \left(\frac{2 \pi y}{\lambda_{2}}\right)
$$

where $\Delta_{1}, \Delta_{2}$ and $\lambda_{1}, \lambda_{2}$ are amplitudes and wave lengths in $\mathrm{x}$ and $\mathrm{y}$ directions,

$$
\Delta_{1}=\Delta_{2}=0.5 \mu m, \quad \lambda_{1}=\lambda_{2}=12.5 \mu m
$$

The size of the wavy surface is $250 \mu \mathrm{m} * 250 \mu \mathrm{m}$, while there are 20 waves in both $\mathrm{x}$ and $y$ directions. 


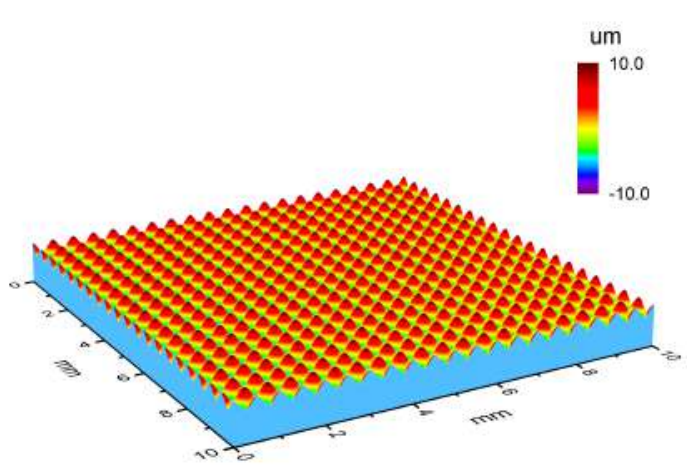

(a)

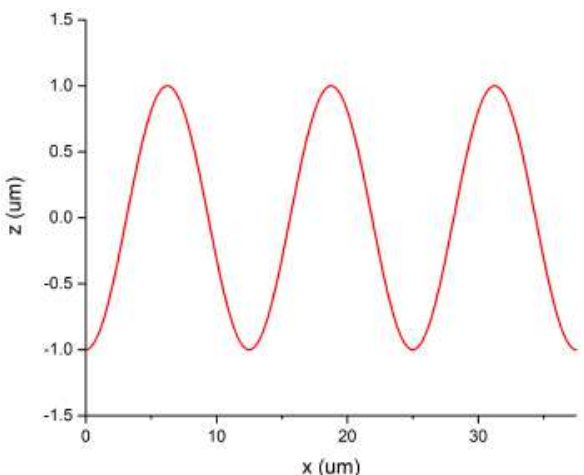

(b)

Fig. 3. The sinusoidal wavy surface:

(a) 3D view; (b) cross-section view.

The elastic modulus of this wavy surface is set to $210 \mathrm{GPa}$, the same as steel. Elastic contacts between this surface and a rigid flat is considered. The three nominal contact pressures $(\overline{\boldsymbol{p}})$ used are $50 \mathrm{MPa}, 100 \mathrm{MPa}$ and $200 \mathrm{MPa}$.

The analytical solutions of maximum contact pressure and real contact area are given in [3]:

$$
p_{\max }=\frac{3 \bar{p}}{2 \pi\left(\frac{3}{8 \pi} \frac{\bar{p}}{p^{*}}\right)^{\frac{2}{3}}}
$$

and

$$
\frac{A_{\text {real }}}{A_{\text {nominal }}}=\pi\left(\frac{3}{8 \pi} \frac{\bar{p}}{p^{*}}\right)^{\frac{2}{3}}
$$

while $\overline{\boldsymbol{p}}$ is the average nominal pressure, and $\boldsymbol{p}^{*}$ is defined as:

$$
p^{*}=2 \pi E^{*} \Delta / \lambda
$$

and $\boldsymbol{E}^{*}$ is the reduced elastic modulus: 


$$
\frac{1}{E^{*}}=\frac{1}{E_{1}}+\frac{1}{E_{2}}
$$

For the SAM calculations, the wavy surface is meshed into six different grid sizes: $64 * 64,128 * 128,256 * 256,512 * 512,1024 * 1024$ and $2048 * 2048$ as input. Then the contacts are solved by the method described in Section 2. The results of real contact area ratios and maximum contact pressures are presented in Tab. 1, Tab. $\mathbf{2}$ and Fig.

4.

Tab. 1. Analytical and SAM results of the maximum contact pressures under different nominal load

\begin{tabular}{|c|c|c|c|c|}
\hline \multirow{2}{*}{$\begin{array}{c}\text { Nominal } \\
\text { pressure (MPa) }\end{array}$} & \multirow{2}{*}{$\begin{array}{c}\text { Analytical } \\
\text { solution (GPa) }\end{array}$} & \multicolumn{2}{|c|}{ SAM solution } & \multirow{2}{*}{ Error (\%) } \\
\hline & & Grid size & Result (GPa) & \\
\hline \multirow{6}{*}{50} & \multirow{6}{*}{10.52} & $64 * 64$ & 3.719 & 64.7 \\
\hline & & $128 * 128$ & 5.637 & 46.4 \\
\hline & & $256 * 256$ & 8.645 & 17.9 \\
\hline & & $512 * 512$ & 11.42 & 8.5 \\
\hline & & $1024 * 1024$ & 10.57 & 0.5 \\
\hline & & $2048 * 2048$ & 10.54 & 0.22 \\
\hline \multirow{6}{*}{100} & \multirow{6}{*}{13.26} & $64 * 64$ & 5.056 & 61.8 \\
\hline & & $128 * 128$ & 7.888 & 40.5 \\
\hline & & $256 * 256$ & 13.42 & 1.3 \\
\hline & & $512 * 512$ & 13.81 & 4.2 \\
\hline & & $1024 * 1024$ & 13.34 & 0.6 \\
\hline & & $2048 * 2048$ & 13.25 & 0.07 \\
\hline \multirow{6}{*}{200} & \multirow{6}{*}{16.71} & $64 * 64$ & 6.872 & 58.9 \\
\hline & & $128 * 128$ & 11.74 & 29.7 \\
\hline & & $256 * 256$ & 18.34 & 9.8 \\
\hline & & $512 * 512$ & 17.04 & 2 \\
\hline & & $1024 * 1024$ & 16.73 & 0.2 \\
\hline & & $2048 * 2048$ & 16.67 & 0.2 \\
\hline
\end{tabular}


Tab. 2. Analytical and SAM results of the real contact area ratio under different nominal load

\begin{tabular}{|c|c|c|c|c|}
\hline \multirow{2}{*}{$\begin{array}{c}\text { Nominal } \\
\text { pressure (MPa) }\end{array}$} & \multirow{2}{*}{$\begin{array}{l}\text { Analytical } \\
\text { solution (\%) }\end{array}$} & \multicolumn{2}{|c|}{ SAM solution } & \multirow{2}{*}{ Error (\%) } \\
\hline & & Grid size & Result (\%) & \\
\hline \multirow{6}{*}{50} & \multirow{6}{*}{0.713} & $64 * 64$ & 2.734 & 283.7 \\
\hline & & $128 * 128$ & 1.733 & 143.2 \\
\hline & & $256 * 256$ & 1.221 & 71.3 \\
\hline & & $512 * 512$ & 0.868 & 21.8 \\
\hline & & $1024 * 1024$ & 0.787 & 8.5 \\
\hline & & $2048 * 2048$ & 0.75 & 5.3 \\
\hline \multirow{6}{*}{100} & \multirow{6}{*}{1.131} & $64 * 64$ & 3.809 & 236.6 \\
\hline & & $128 * 128$ & 2.441 & 115.8 \\
\hline & & $256 * 256$ & 1.678 & 48.4 \\
\hline & & $512 * 512$ & 1.326 & 17.2 \\
\hline & & $1024 * 1024$ & 1.228 & 7.9 \\
\hline & & $2048 * 2048$ & 1.184 & 4.6 \\
\hline \multirow{6}{*}{200} & \multirow{6}{*}{1.796} & $64 * 64$ & 5.469 & 204.5 \\
\hline & & $128 * 128$ & 3.222 & 79.4 \\
\hline & & $256 * 256$ & 2.289 & 27.4 \\
\hline & & $512 * 512$ & 2.055 & 14.5 \\
\hline & & $1024 * 1024$ & 1.932 & 7.5 \\
\hline & & $2048 * 2048$ & 1.875 & 4.4 \\
\hline
\end{tabular}




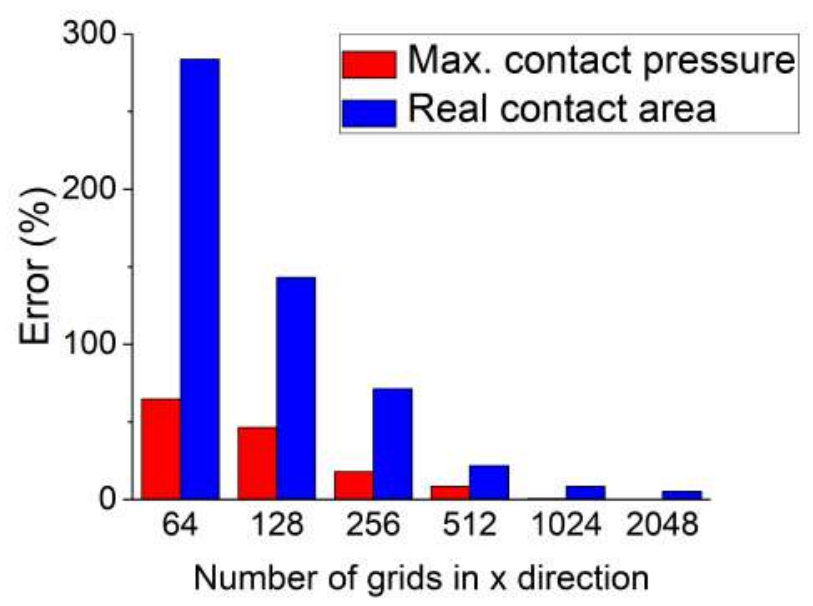

(a)

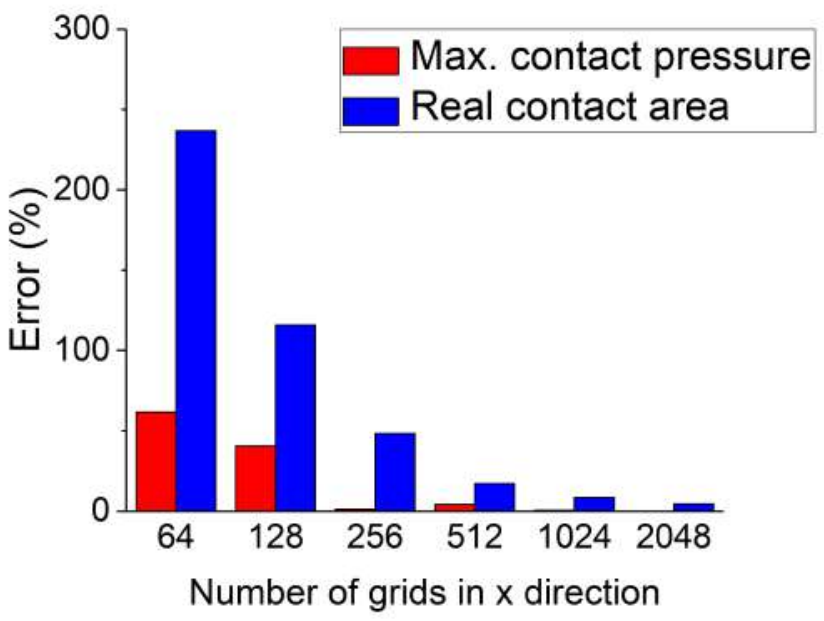

(b) 


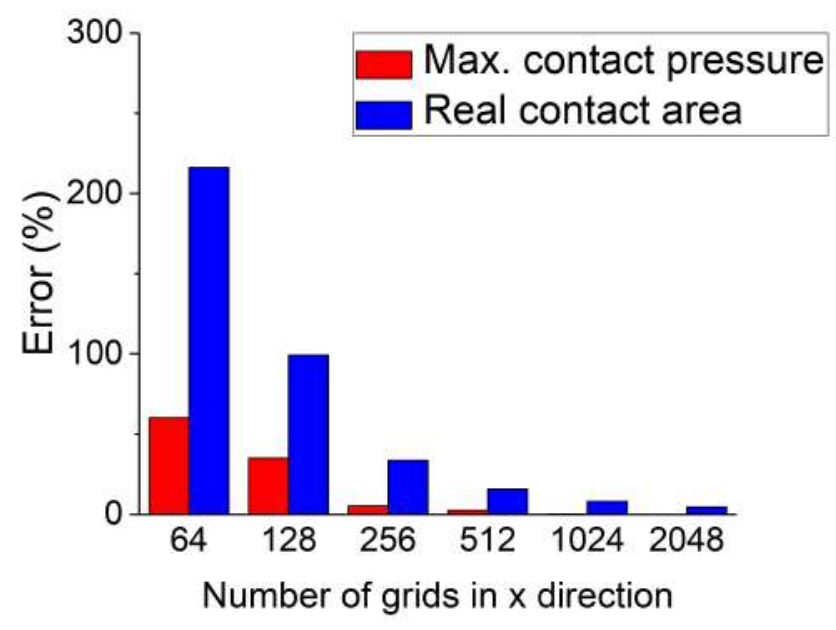

(c)

Fig. 4. Relative errors of the SAM results compared with the analytical solutions with different nominal loads: (a) $50 \mathrm{MPa}$; (b) $100 \mathrm{MPa}$; (c) $200 \mathrm{MPa}$.

Clearly, the accuracy of the SAM results is sensitive to the resolutions of input: the higher the input resolution is, the smaller the relative error will be. Both maximum contact pressures and real contact areas converge to the analytical solution when the input resolutions go high. However, it is worth noting that the converging rate of the contact pressures and real contact areas are significantly different. Take the 100 MPa case for example: both starting from very large errors for the $64 * 64$ case, the maximum contact pressure can be predicted by SAM with rather satisfactory accuracy under the resolution of $256 * 256$ (1.26\% relative error in comparison with the analytical solution), while the real contact area result under the same resolution is rather poor ( $48.4 \%$ relative error). Even with the highest input resolution of $2048 * 2048$, the relative error of the real contact area remains $4.61 \%$, in contrast to the $0.07 \%$ for the maximum contact pressure. Similar trends are also observed in the cases with other nominal contact pressures.

This phenomenon is caused by the different ways SAM calculates the contact pressure and real contact area. The contact pressures are calculated directly by SAM, as described in Section 2, while the errors come mainly from the inadequate input information. This issue can be resolved by increasing the resolution of input. The calculation of the real contact area is, however, indirect: the algorithm firstly 
determines the contact pressures on each grid, and the grids with non-zero contact pressures will be marked as "in contact". Then the contact area will be calculated by the total area of the grids in contact. However, using rectangle grid size, the grids will usually overly cover the contact boundaries. This "over covering" effect leads to larger errors in calculating the real contact area values.

An elaborated demonstration is presented in Fig. 5, which gives a close look at the calculated results of local contact on a single summit of the wavy surface, under 100 MPa nominal contact pressure. The resolutions of input being used are $256 * 256$, $512 * 512,1024 * 1024,2048 * 2048$ respectively. It appears that with low input resolution $(256 * 256)$ while the grid sizes are large, the over-covering effect is quite severe (Fig. 5a). In this case, a rectangle shape is considered by SAM as the real contact in this spot, while the actual contact area shape is a circle. Consequently, a large error occurs here. With higher input resolutions, the sizes of grids become smaller, the over-covering effect become less severe, and more accurate coverages are achieved (Fig. 5b, $\mathbf{5 c}$ and $\mathbf{5 d}$ ). This provides a greater accuracy of the numerical results. Note that this over-covering effect only exists in calculating the real contact area, while the calculation of the contact pressures is hardly affected by this. The effect of this is that SAM shows much higher sensitivity to input grid size when calculating the real contact area than the contact pressure.

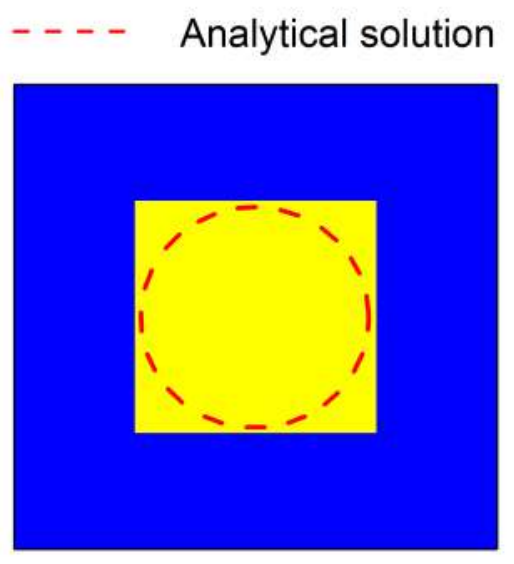

(a)

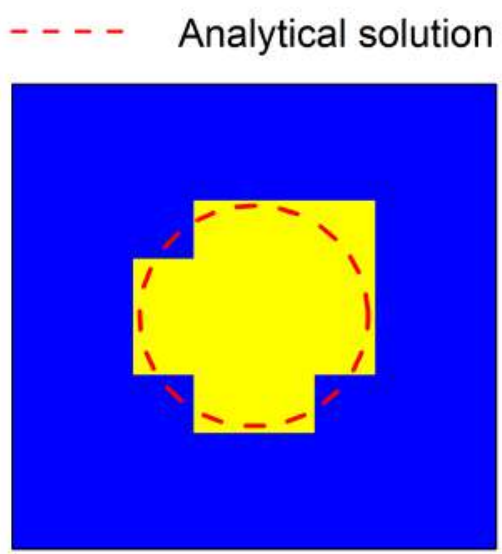

(b) 


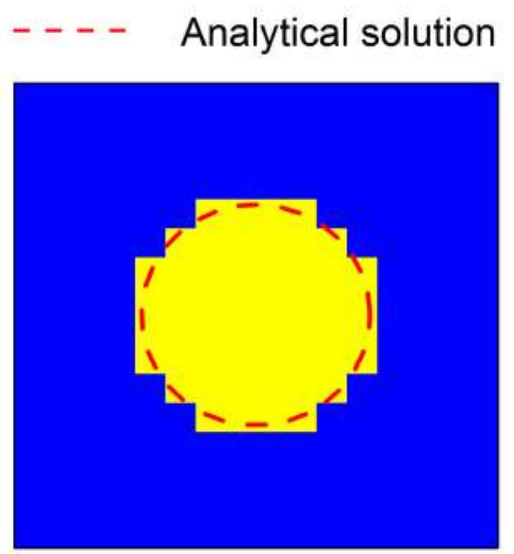

(c)
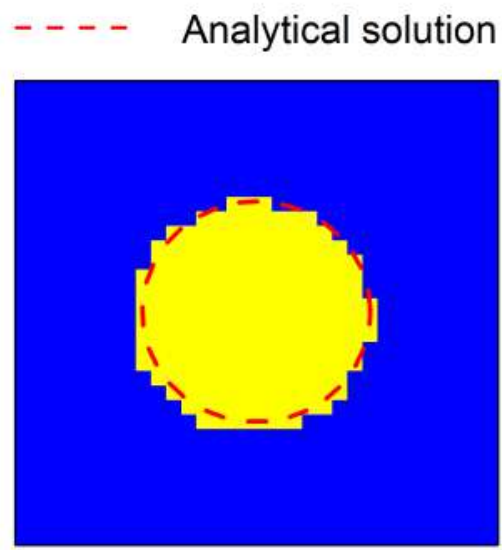

(d)

Fig. 5. SAM results of real contact areas with different resolutions of input (yellow grids are in contact and blue grids are not in contact): (a) 256*256; (b) 512*512; (c) $1024 * 1024$; (d) $2048 * 2048$.

It should be noted that a resolution of $2048^{*} 2048$ is quite high, thus may not be feasible in some situations. Usually, much lower resolutions are applied in contact analyses [14]-[2]. Therefore, it is possible that the calculated real contact areas by SAM are not reliable in many cases due to the inadequate resolutions of input.

An extrapolation method is proposed to acquire more accurate contact areas when further improving the input resolution is impossible or inconvenient. A threeparameter decayed exponential formula is used to fit the data points:

$$
A=k e^{-\frac{x}{t}}+A_{0}
$$

In this formula, $\boldsymbol{A}$ is the real contact area calculated by SAM, $\mathrm{x}$ is the number of grids in $\mathrm{x}$ or $\mathrm{y}$ direction, and $\boldsymbol{k}, \boldsymbol{t}$ and $\boldsymbol{A}_{\mathbf{0}}$ are the three fitting parameters. The term $\boldsymbol{k} \boldsymbol{e}^{-\frac{\boldsymbol{x}}{\boldsymbol{t}}}$ can be regarded as the error term caused by the over-covering effect. When the resolution term $x$ goes larger, the value of this term will decrease, and eventually 
converge to zero when the grid size goes to infinite. Then the term $\boldsymbol{A}_{\mathbf{0}}$ will be the predicted real contact area.

This technique is applied to the real contact area results of the wavy surface, see Fig. 6. The exponential functions achieve satisfactory fitting accuracies for all three contact cases. For each data set, the six data points fall fairly close to the fitting curve. In addition, the fitting curves converge accurately to the analytical solution. See Tab. 3.

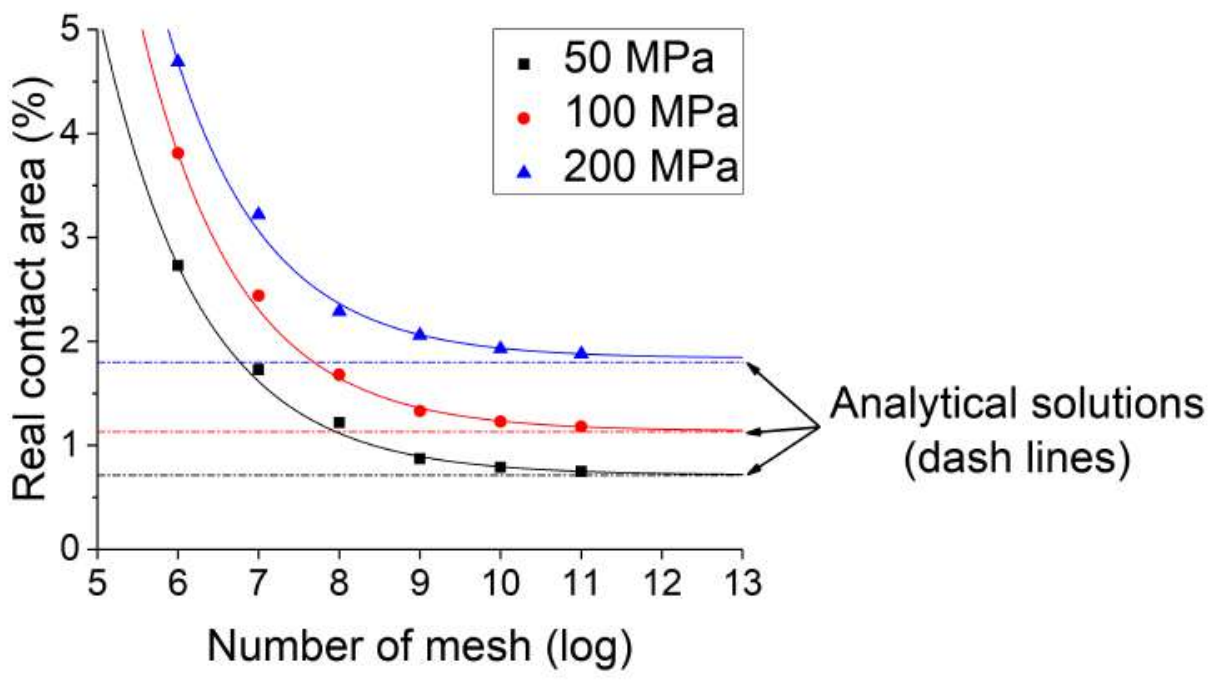

Fig. 6. Exponential extrapolation of real contact area of the wavy surfaces.

Tab. 3. Comparison of Extrapolation results and analytical results.

\begin{tabular}{cccc}
\hline $\begin{array}{c}\text { Nominal pressure } \\
(\mathrm{MPa})\end{array}$ & $\begin{array}{c}\text { Analytical } \\
\text { solution }\end{array}$ & $\begin{array}{c}\text { Extrapolation } \\
\text { results }\end{array}$ & Error (\%) \\
\hline 50 & 0.713 & 0.714 & 0.14 \\
100 & 1.131 & 1.137 & 0.45 \\
200 & 1.796 & 1.838 & 2.33 \\
\hline
\end{tabular}

Based on the former discussion, it can be concluded that real contact area results are more sensitive to the resolutions of input surface, and therefore deserve extra attention. Exponential extrapolation can be employed to predict the real contact 
area value based on a series of results from different input resolutions. This technique can be further applied to the real rough surface contact calculation, which is elaborated in the following subsection.

\subsection{Real rough surfaces}

In this subsection, the elasto-plastic contact between a $5 \mathrm{~mm}$ radius ball and a grinded ring (Fig. 7) is studied to investigate the sensitivity of the SAM results with respect to the resolutions of input. Both the ball and the ring are made of 52100 bearing steel. The mechanical properties are shown in Tab. 4.

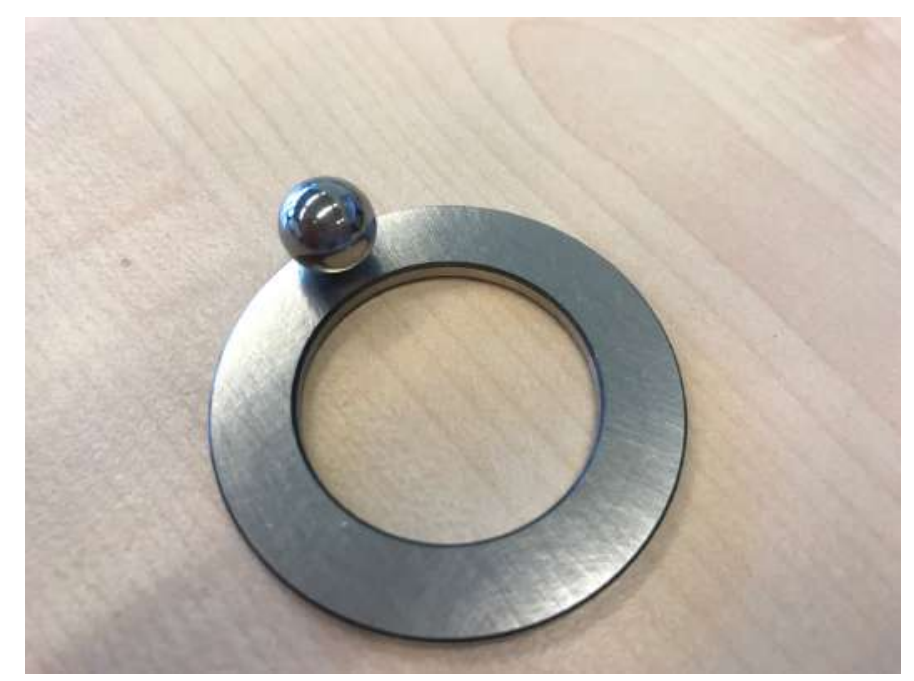

Fig. 7. Ball and flat surfaces samples.

Tab. 4. Mechanical properties of 52100 steel.

\begin{tabular}{cc}
\hline Parameter & Value \\
\hline Elastic modulus (GPa) & 210 \\
Poisson's ratio & 0.3 \\
Hardness (GPa) & 6 \\
\hline
\end{tabular}

Two surface conditions are considered: before and after running in. The running in process was carried out by a pin on disk testing machine. The surface data were acquired by a confocal microscope SENSOFAR S Neox. The measuring domains are 
originally $264.2 \mu \mathrm{m} * 264.2 \mu \mathrm{m}$, with $2048 * 2048$ grid size, as presented in Fig. 8 and Fig. 9.

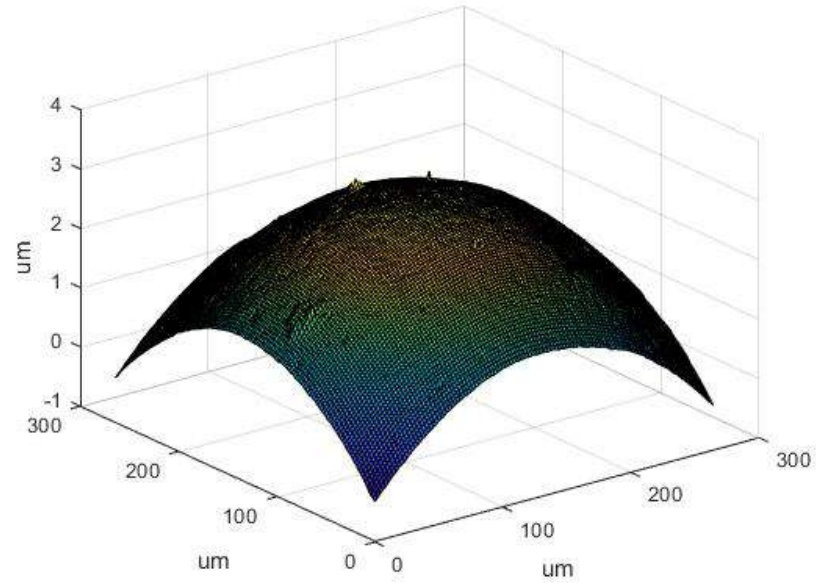

(a)

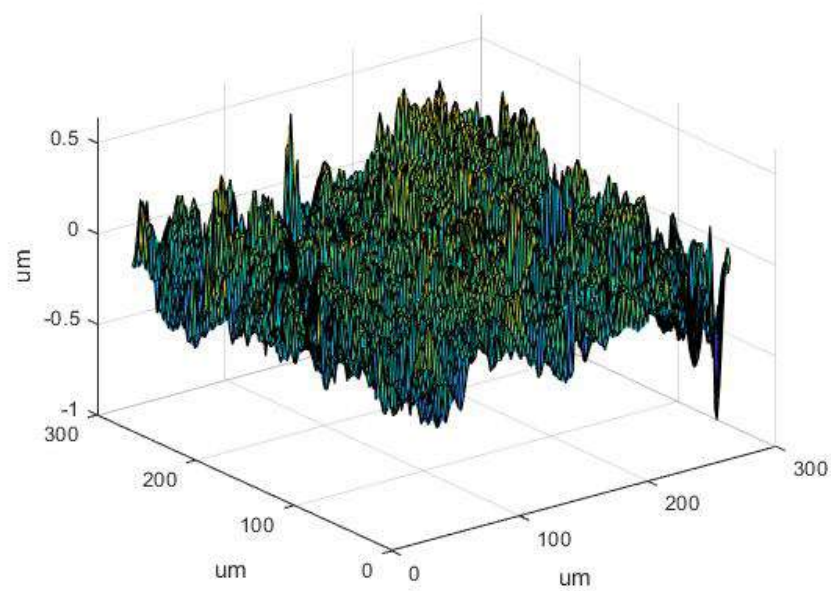

(b)

Fig. 8. Surface profiles of samples before running in: (a) the ball; (b) the ring. 


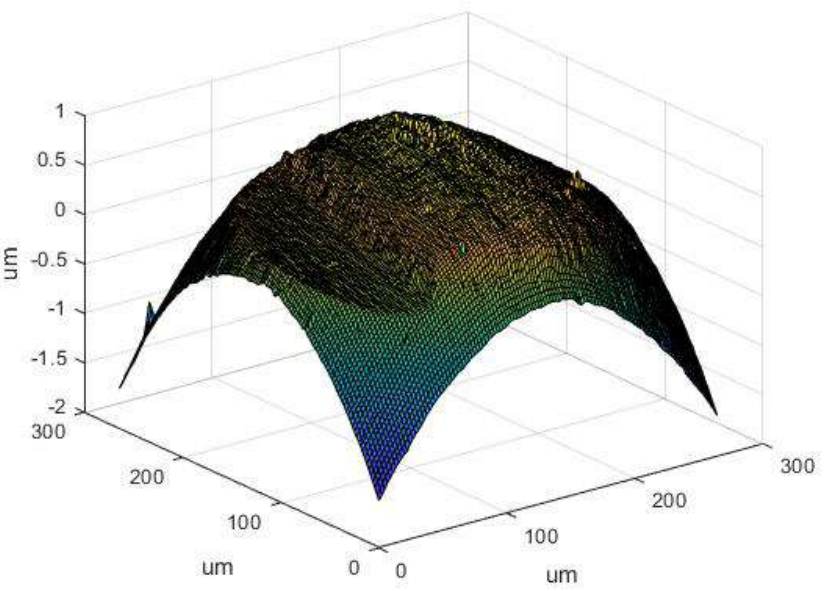

(a)

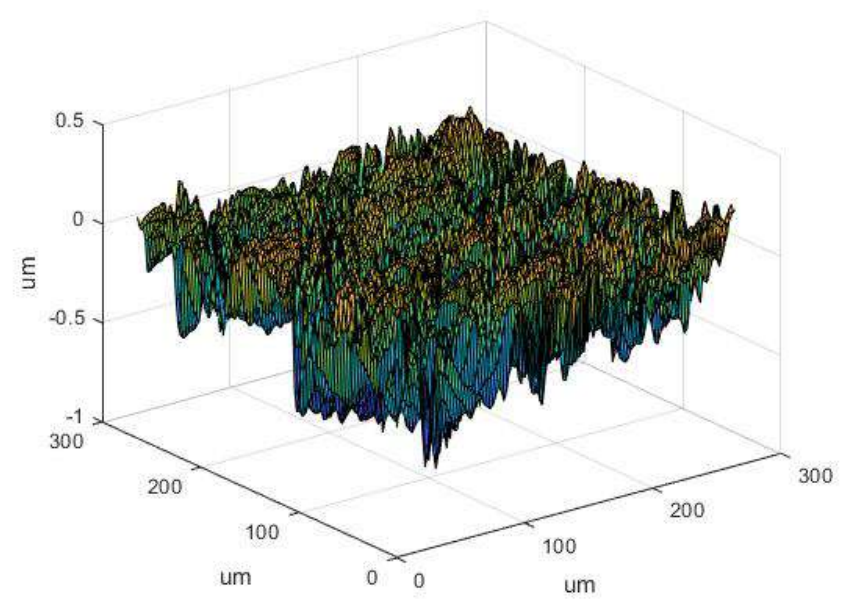

(b)

Fig. 9. Surface profiles of samples after running in: (a) the ball; (b) the ring.

In order to investigate the effect of resolution, the surface data are scaled into five different lower resolutions $64 * 64,128 * 128,256 * 256,512 * 512,1024 * 1024$ by the bilinear scaling algorithm. Together with the original $2048 * 2048$ data, six different resolutions of input are acquired. Three different normal loads are applied, which are $2.5 \mathrm{~N}, 5 \mathrm{~N}$ and $10 \mathrm{~N}$ respectively. Considering the elasto-plastic property of the 
material, the maximum contact pressure is set to $6 \mathrm{GPa}$, the same as the hardness. A single elastic-perfect-plastic curve is employed in the calculation.

The calculated real contact areas with different surface input grid sizes are shown in Fig. 10. The results indicate that, for the samples before running in, the real contact areas converge relatively well while, for the samples after running in, the results show substantial differences even with high input resolutions. Therefore, the accuracies of the calculated real contact area values remain questionable.

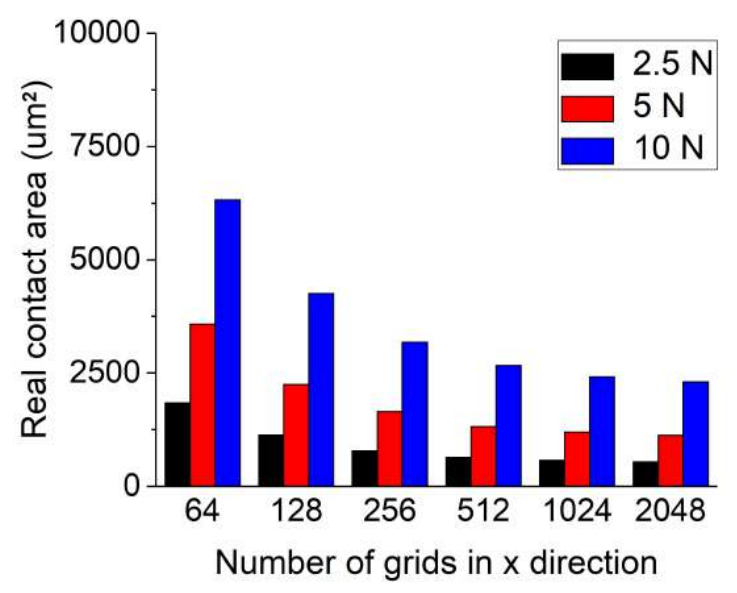

(a)

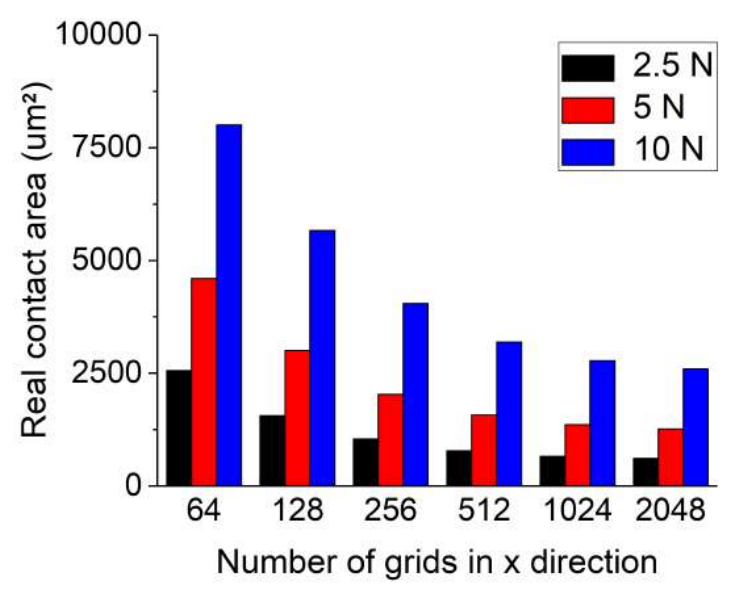

(b)

Fig. 10. Real contact areas calculated by SAM with different resolutions of input:

(a) before running in and (b) after running in. 
The real contact areas are predicted by the fitting technique proposed in Subsection 3.1, see Fig. 11. The results show that, for both cases, the results from the different input resolutions fall on the exponential curves nicely. The predicted results are compared with the SAM results with the highest resolution (2048*2048) in Tab. 5.

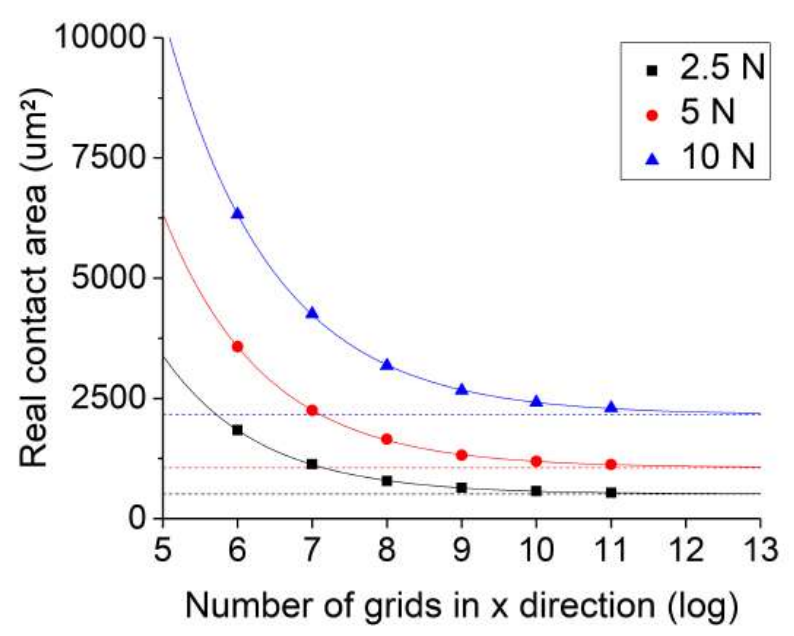

(a)

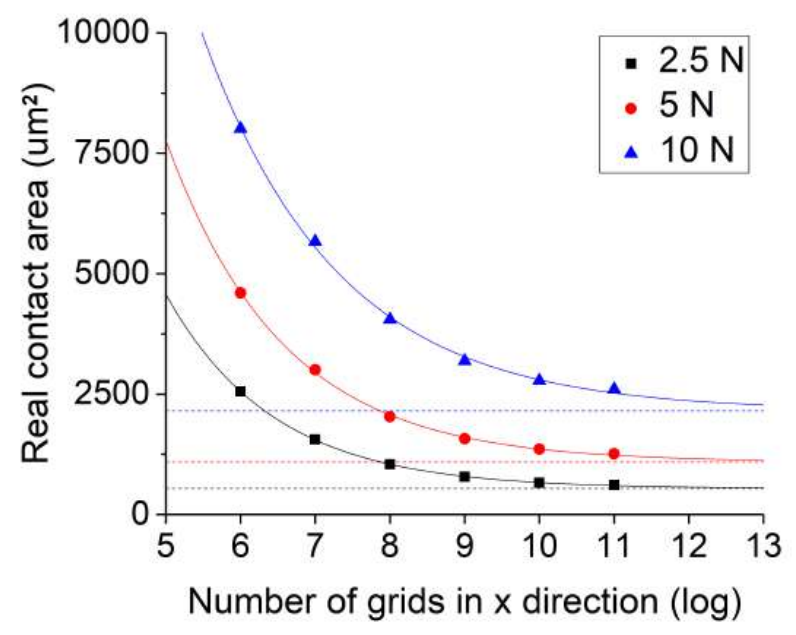

(b)

Fig. 11. Exponential extrapolation of real contact areas of the samples: (a) before running in; (b) after running in. 
Tab. 5. Comparison of real contact areas: SAM results and extrpolation results of samples.

\begin{tabular}{ccccc}
\hline Samples & Normal load (N) & $\begin{array}{c}\text { SAM results } \\
(2048 * 2048)\end{array}$ & $\begin{array}{c}\text { Extrapolation } \\
\text { results }\end{array}$ & Error (\%) \\
\hline $\begin{array}{c}\text { Before running } \\
\text { in }\end{array}$ & 2.5 & 540.1 & 509.9 & 5.9 \\
& 5 & 1126.2 & 1063.1 & 5.9 \\
\hline \multirow{2}{*}{ After running in } & 10 & 2301.2 & 2159.6 & 6.6 \\
\hline & 5 & 613.9 & 538.3 & 14.1 \\
& 10 & 1262.4 & 1086.9 & 16.2 \\
\hline
\end{tabular}

The extrapolation results confirm the concerns: for the case after the running in, the SAM results do not converge well even with the highest input resolution. This issue is particularly severe for the contact with $10 \mathrm{~N}$ normal load: the data point is obviously deviated from the converging line. A $20.8 \%$ of difference is observed between the SAM result and the predicted result of extrapolation.

This phenomenon can also be explained by the over-covering effect. The contact pressure distributions of both cases with $10 \mathrm{~N}$ normal load are plotted in Fig. 12 (2048*2048 in resolution). For the contact of samples after running in, the contact pressure distribution is more dispersed. This leads to more local contacts with very small contact areas. For these small local contact areas, the grid sizes being used are not small enough to precisely cover the boundary and therefore lead to a severe over-covering effect. 


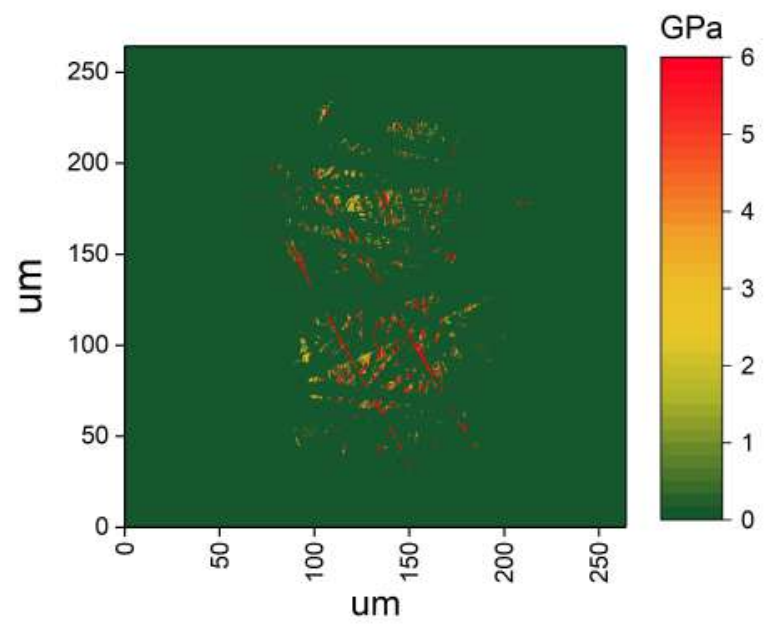

(a)

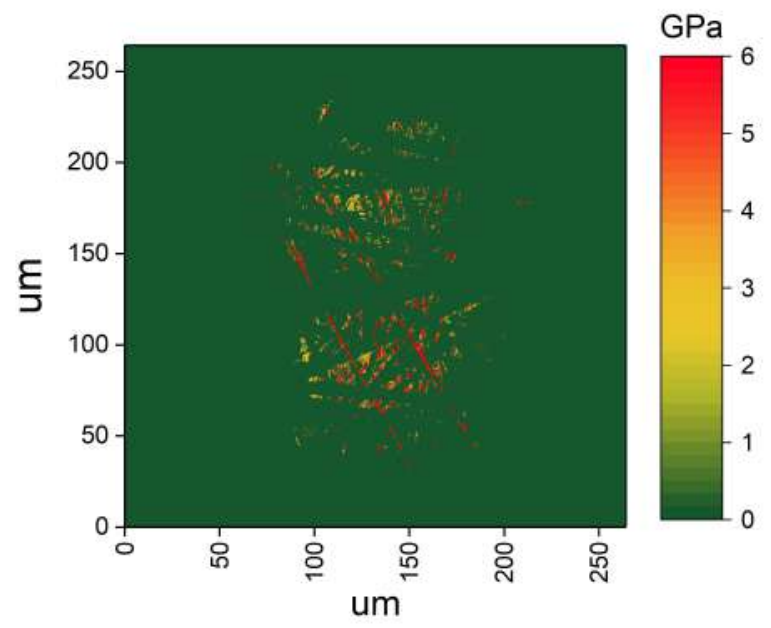

(b)

Fig. 12. Contact pressure distribution of the samples: (a) before running in; (b) after running in.

A close look at one of the local contacts is taken in Fig. 13. The calculated results with different input resolutions $(256 * 256,512 * 512,1024 * 1024,2048 * 2048)$ are shown. These two adjacent contact areas are small in area $(\sim 1 \mu \mathrm{m} * 1 \mu \mathrm{m})$. For the $256 * 256$ 
case (Fig.13a), only two grids are included, which leads to a very coarse covering. A severe over-covering effect occurs and a very large error will occur. With an increase of input grid size, a finer and finer covering is achieved (Fig.13b, 13c and 13d). As can be easily seen, the calculated contact areas are decreasing. However, even in the highest resolution $(2048 * 2048)$ case, there is still only a very limited number of grids included in the contact area, and the boundaries of contact are still relatively coarse. Therefore, the over-covering effect is probably still substantial. This causes the $20.8 \%$ difference between the SAM result and predicted result of extrapolation.

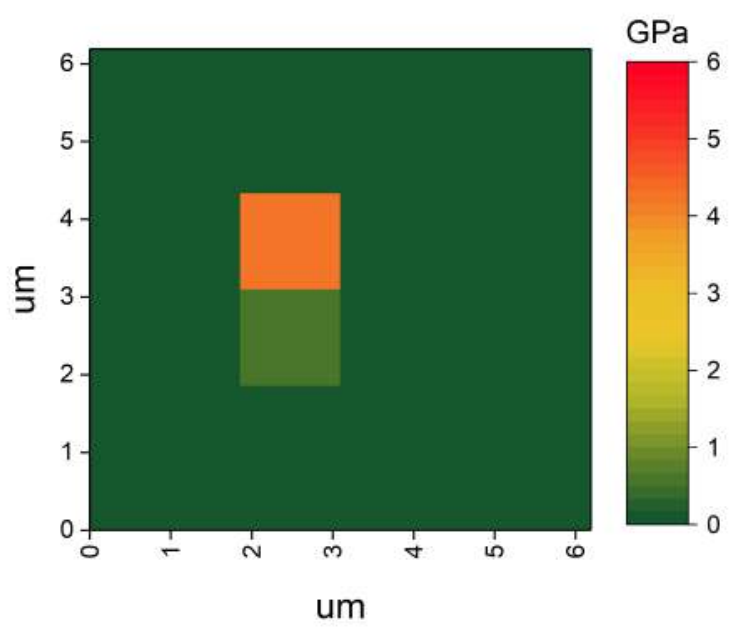

(a)

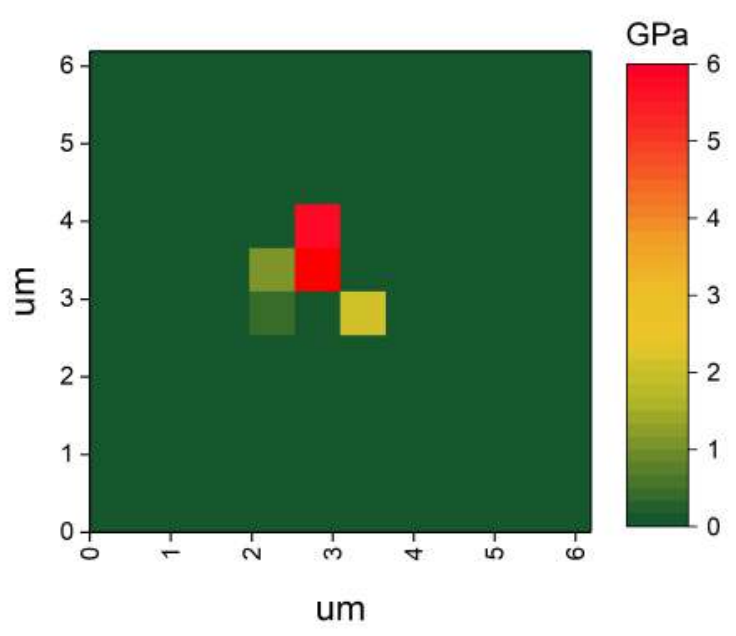

(b) 


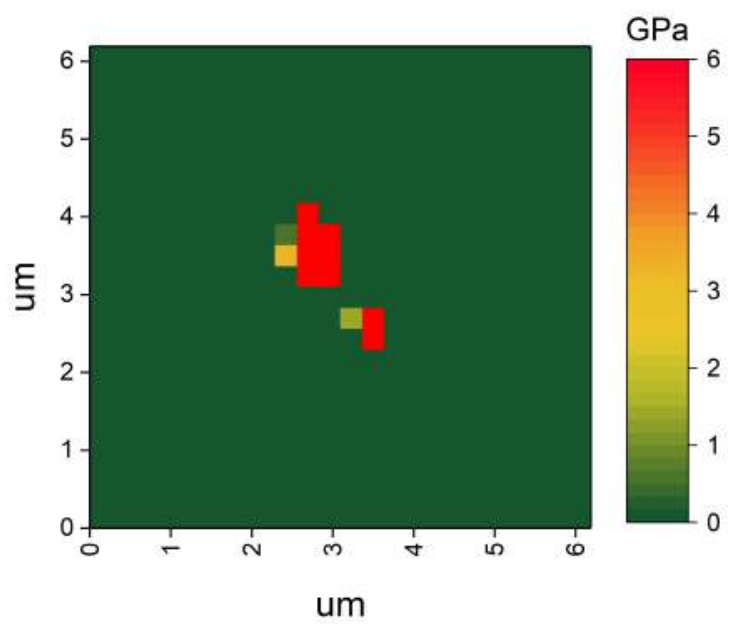

(c)

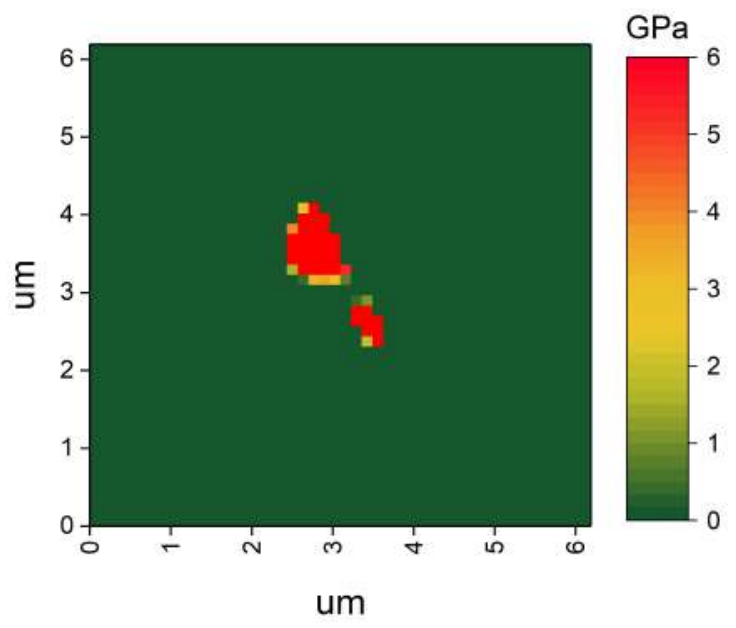

(d)

Fig. 13. Local contact results calculated with different input resolutions: (a) 256*256; (b) 512*512; (c) 1024*1024; (d) 2048*2048.

4. Conclusions 
We have demonstrated, for the first time, the sensitivity of SAM results of rough surface contact to the resolutions of input. The primary conclusions are summarized below.

(1) Compared to the maximum contact pressure, the real contact area results calculated by SAM are particularly sensitive to the input grid size of the surfaces. The converging rates of the real contact area results are significantly lower than the maximum contact pressure results.

(2) An over-covering effect occurs when SAM calculates the real contact area. This is the main reason for the high sensitivity of the real contact area results to the resolutions of input. The rectangle shaped grids usually overly cover the boundaries of the contact area. The larger the size of the grid is, the more severe this effect will be.

(3) In real rough surface contacts, the "over-covering" effect of SAM can be severe, especially in the cases where the contact pressures are distributed dispersedly. In these cases, local contact areas are quite small: similar or even smaller than the size of the grids. As a consequence, the calculated real contact area may significantly deviate from the real values.

(4) The exponential extrapolation can be employed to predict the real contact area, when further improvement the grid size of input is difficult. This method depends solely on the inputs, i.e. the calculation results from coarser meshes. Therefore, it is suggested that feasibly finer meshes should be used.

\section{References}

[1] Mo, Y., Turner, K. T., \& Szlufarska, I. (2009). Friction laws at the nanoscale. Nature, 457(7233), 1116.

[2] Stachowiak, G. W. (Ed.). (2006). Wear: materials, mechanisms and practice. John Wiley \& Sons.

[3] Renouf, M., Massi, F., Fillot, N., \& Saulot, A. (2011). Numerical tribology of a dry contact. Tribology International, 44(7-8), 834-844.

[4] Polonsky, I. A., \& Keer, L. M. (1999). A numerical method for solving rough contact problems based on the multi-level multi-summation and conjugate gradient techniques. Wear, 231(2), 206-219.

[5] Love, A. E. H., Mathematical Theory of Elasticity (Cambridge, 1927), 193. 
[6] Bjorklund, S., Andersson, S., 1994. A numerical method for real elastic contacts subjected to normal and tangential loading. Wear 179, 117-122.

[7] O'Sullivan, T. C., \& King, R. B. (1988). Sliding contact stress field due to a spherical indenter on a layered elastic half-space. Journal of Tribology, 110(2), 235-240.

[8] Nogi, T., \& Kato, T. (1997). Influence of a hard surface layer on the limit of elastic contact-Part I: Analysis using a real surface model. Journal of Tribology, 119(3), 493-500.

[9] Cai, S., \& Bhushan, B. (2005). A numerical three-dimensional contact model for rough, multilayered elastic/plastic solid surfaces. Wear, 259(7-12), 14081423.

[10] Yu, C., Wang, Z., \& Wang, Q. J. (2014). Analytical frequency response functions for contact of multilayered materials. Mechanics of Materials, 76, 102-120.

[11] Jacq, C., Nelias, D., Lormand, G., \& Girodin, D. (2002). Development of a threedimensional semi-analytical elastic-plastic contact code. Journal of Tribology, 124(4), 653-667.

[12] Chiu, Y. P. (1977). On the stress field due to initial strains in a cuboid surrounded by an infinite elastic space. Journal of Applied Mechanics, 44(4), 587-590.

[13] Chiu, Y. P. (1978). On the stress field and surface deformation in a half space with a cuboidal zone in which initial strains are uniform. Journal of Applied Mechanics, 45(2), 302-306.

[14] Liu, S., \& Wang, Q. (2005). Elastic fields due to eigenstrains in a half-space. Journal of Applied Mechanics, 72(6), 871-878.

[15] Jin, X., Keer, L. M., \& Wang, Q. (2011). A closed-form solution for the Eshelby tensor and the elastic field outside an elliptic cylindrical inclusion. Journal of Applied Mechanics, 78(3), 031009.

[16] Zhou, Q., Jin, X., Wang, Z., Wang, J., Keer, L. M., \& Wang, Q. (2015). Numerical implementation of the equivalent inclusion method for 2D arbitrarily shaped inhomogeneities. Journal of Elasticity, 118(1), 39-61.

[17] Bosman, R., Hol, J., \& Schipper, D. J. (2011). Running-in of metallic surfaces in the boundary lubrication regime. Wear, 271(7-8), 1134-1146.

[18] Bosman, R., \& Schipper, D. J. (2011). Running-in of systems protected by additive-rich oils. Tribology Letters, 41(1), 263-282.

[19] Bosman, R., \& Schipper, D. J. (2011). Transition from mild to severe wear including running in effects. Wear, 270(7-8), 472-478.

[20] Rajasekaran, R., \& Nowell, D. (2006). Fretting fatigue in dovetail blade roots: Experiment and analysis. Tribology International, 39(10), 1277-1285. 
[21] Done, V., Kesavan, D., Chaise, T., \& Nelias, D. (2017). Semi analytical fretting wear simulation including wear debris. Tribology International, 109, 1-9.

[22] Gallego, L., Fulleringer, B., Deyber, S., \& Nelias, D. (2010). Multiscale computation of fretting wear at the blade/disk interface. Tribology International, 43(4), 708-718.

[23] Chen, W. W., \& Wang, Q. J. (2008). A numerical model for the point contact of dissimilar materials considering tangential tractions. Mechanics of Materials, 40(11), 936-948.

[24] Chen, P., \& Chen, S. (2013). Partial slip contact between a rigid punch with an arbitrary tip-shape and an elastic graded solid with a finite thickness. Mechanics of Materials, 59, 24-35.

[25] Wang, Z., Jin, X., Keer, L. M., \& Wang, Q. (2013). Novel model for partial-slip contact involving a material with inhomogeneity. Journal of Tribology, 135(4), 041401.

[26] Wang, Z., Jin, X., Zhou, Q., Ai, X., Keer, L. M., \& Wang, Q. (2013). An efficient numerical method with a parallel computational strategy for solving arbitrarily shaped inclusions in elastoplastic contact problems. Journal of Tribology, 135(3), 031401.

[27] Johnson, K. L., Greenwood, J. A., \& Higginson, J. G. (1985). The contact of elastic regular wavy surfaces. International Journal of Mechanical Sciences, 27(6), 383-396. 



\title{
Paper C
}

\author{
Wang, C., Gojzewski, H., \& Schipper, D. J. (2020). A multi-technique \\ characterization of the tribofilm formed by a fully formulated CVT fluid. \\ Tribology International, 146, 106201.
}





\title{
A multi-technique characterization of the tribofilm formed by a fully formulated CVT fluid
}

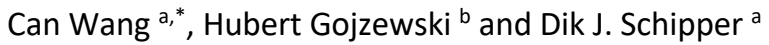 \\ a Laboratory for Surface Technology and Tribology, Department of Engineering Technology, \\ University of Twente, 7522 NB Enschede, The Netherlands \\ ${ }^{\mathrm{b}}$ Materials Science and Technology of Polymers, Faculty of Science and Technology, \\ University of Twente, Drienerlolaan 5, 7522 NB Enschede, The Netherlands \\ *Correspondence: c.wang-1@utwente.nl
}

\begin{abstract}
In this work, the morphology, thickness, chemical composition and mechanical properties of the tribofilm formed by a fully formulated CVT fluid are investigated by multiple techniques and linked to the frictional and wear characteristics of a pin-ondisc tribosystem. It is found that the tribosystem shows higher friction and wear when tested at $150{ }^{\circ} \mathrm{C}$ than at $80{ }^{\circ} \mathrm{C}$. The main reason is that although the morphology and thickness are similar, the tribofilms formed at different temperatures have different chemical compositions and mechanical properties. The tribofilm formed at $150{ }^{\circ} \mathrm{C}$ is Fe richer and has greater hardness and shear strength, which leads to a higher tribochemical wear rate and a greater interfacial shear force.

Keywords: tribofilm; chemical composition; mechanical properties; AFM
\end{abstract}

\section{Nomenclature}

$\begin{array}{lll}\boldsymbol{A}_{\boldsymbol{c}} & \text { Contact area (hertzian) } & \mathrm{m}^{2} \\ \boldsymbol{A}_{\boldsymbol{p}} & \text { Projected area of the remaining deformation } & \mathrm{m}^{2} \\ \boldsymbol{E} & \text { Young's modulus of tribofilm } & \mathrm{Pa} \\ \boldsymbol{E}_{\boldsymbol{c}} & \text { Young's modulus of cantilever } & \mathrm{Pa}\end{array}$




\begin{tabular}{|c|c|c|}
\hline $\boldsymbol{F}_{\boldsymbol{a d v}}$ & Adhesion force & $\mathrm{N}$ \\
\hline$F_{D M T}$ & Applied load based on DMT model & $\mathrm{N}$ \\
\hline $\boldsymbol{F}_{\boldsymbol{l}}$ & Lateral load & $\mathrm{N}$ \\
\hline $\boldsymbol{F}_{\boldsymbol{n}}$ & Normal force & $\mathrm{N}$ \\
\hline $\boldsymbol{G}_{\boldsymbol{c}}$ & Shear modulus of cantilever & $\mathrm{Pa}$ \\
\hline $\boldsymbol{h}$ & Height of tip & $\mathrm{m}$ \\
\hline $\mathrm{H}$ & Hardness of tribofilm & $\mathrm{Pa}$ \\
\hline $\boldsymbol{k}_{\boldsymbol{l}}$ & Cantilever spring constant (lateral) & $\mathrm{N} / \mathrm{m}$ \\
\hline $\boldsymbol{k}_{n}$ & Cantilever spring constant (normal) & $\mathrm{N} / \mathrm{m}$ \\
\hline $\boldsymbol{l}$ & $\begin{array}{l}\text { Length of cantilever (from the end of the chip to the apex of the } \\
\text { tip) }\end{array}$ & $\mathrm{m}$ \\
\hline $\boldsymbol{R}$ & Radius of tip & $\mathrm{m}$ \\
\hline$S_{l}$ & Sensitivity of AFM optical system (lateral) & $\mathrm{V} / \mathrm{m}$ \\
\hline$S_{n}$ & Sensitivity of AFM optical system (normal) & $\mathrm{V} / \mathrm{m}$ \\
\hline $\boldsymbol{t}$ & Thickness of cantilever & $\mathrm{m}$ \\
\hline$V_{l}$ & Photo-potential of PSPD (lateral) & V \\
\hline$V_{n}$ & Photo-potential of PSPD (normal) & V \\
\hline $\boldsymbol{w}$ & Width of cantilever & $\mathrm{m}$ \\
\hline $\boldsymbol{v}$ & Poisson's ratio & 1 \\
\hline$\delta$ & Indentation depth & $\mathrm{m}$ \\
\hline$\tau$ & Shear strength of tribofilm & $\mathrm{Pa}$ \\
\hline
\end{tabular}

\section{Introduction}

Tribofilms have long been known to be crucial in the friction and wear control of boundary lubrication, where film thicknesses are not sufficient to separate surfaces 
and asperity to asperity contacts occur. Generated by chemical reactions between additives and contact surfaces, tribofilms are constantly formed and removed in boundary lubrication and serve as self-sacrificing layers. A variety of additives are applied for the formation of tribofilms with different friction and wear performances, while Zinc dithiophosphates (ZDDPs) are arguably the most commonly used additive. In the past decades, a huge number of research papers has been published investigating the role that tribofilms play in the tribosystem and the mechanisms of their friction and wear control.

Morphology, thickness and chemical composition of the tribofilms have been investigated by researchers for decades, due to their close linkage to the frictional and wear characteristics of the tribosystem. Various surface analytical techniques have been applied in this field. Scanning electron microscopy (SEM) [1]-[6] and atomic force microscopy (AFM) [7]-[10] are extensively employed for the morphology of the tribofilms. Gosvami et al. [11] even performed in-situ AFM observations of the ZDDP tribofilm formed under different contact pressures and temperatures. A focused ion beam microscope (FIB) is frequently used to measure the thickness of the tribofilms [12]-[16], by providing straightforward cross-section profile images. As to the chemical composition of tribofilms, energy dispersive X-ray spectroscopy (EDX) is commonly employed to carry out the qualitative analysis in combination with SEM [17]-[21] X-ray photoelectron spectroscopy (XPS) and X-ray absorption near edge structure (XANES) also serve as powerful tools to analyze the chemical state of elements in tribofilms [22]-[28].

Further, the mechanical properties of the tribofilms are also of great concern. In microscale and nanoscale tribology, it is proposed that the friction is controlled by the effective shear strength of the contacting surface layers and the real contact area [29][30][31]. In boundary lubrication with existing tribofilms, this theory is also widely supported by literature. Mortier et al. [32] suggested that some tribofilms have low shear strength properties and allowed shearing within the film structure itself, and therefore led to lower friction. The shear strength of the ZDDP tribofilm were linked to their friction modifying effects by different research groups [33]-[36]. Therefore, investigating the mechanical properties of tribofilms helps understanding the friction in layered boundary lubrication. However, due to the small thicknesses (usually 50-200 nm) and the non-uniform distribution of the tribofilms, research in this area has been rather limited. The nano-hardness of tribofilms, serving as an indirect indication of the shear strength of tribofilms, has been investigated by researchers. Bec et al. [37] performed in-situ imaging of different tribofilms and 
found that the hardness of the tribofilms varied in depth. For ZDDP, the hardness started from about $1 \mathrm{GPa}$ on the top surface and increased to $3 \mathrm{GPa}$ at $20 \mathrm{~nm}$ depth. Aktary et al. [38] reported that the hardness of ZDDP tribofilms formed on AISI 52100 steel ranged from 2.3 to $5.0 \mathrm{GPa}$. Pereira et al. [39] measured the hardness of ZDDP tribofilms at different temperatures and found a 2-4.5 GPa range. Recently, Ueda et al. [40] reported hardness values of ZDDP films of 2.4 and $3.3 \mathrm{GPa}$ after $25 \mathrm{~min}$ and $6 \mathrm{~h}$ rubbing. As to the researches on shear strength of the tribofilms, Pidduck and Smith [41] proposed the idea of employing AFM and lateral force microscopy (LFM) to study the lateral force of the tribofilm. Kano and Yasuda [42] employed the AFM to study the shearing forces of the ZDDP tribofilm. However, only the electrical signals were presented, without being translated into forces.

In this work, the connection between the friction and wear characteristics of the tribosystem and the properties of the tribofilm (morphological, chemical, mechanical, etc.) are investigated. The tribofilm being studied is formed by the fully formulated Shell NS-3 oil. First, the friction and wear characteristics of the system are studied by pin-on-disc tests. The post-test surfaces are subsequently analyzed by AFM, FIB-SEM and XPS for the morphology, thickness and chemical composition of the tribofilms respectively. Nanoscale hardness and Young's modulus are calculated employing a mechanical contact model, based on the evaluation of collected AFM images and force-indentation depth curves. The shear strengths of the tribofilms are further measured by the AFM running under LFM mode.

\section{Experimental design}

\subsection{Pin-on-disc tribometer}

The lubricated metal to metal sliding contacts at a temperature range of $80-150{ }^{\circ} \mathrm{C}$ were evaluated by a pin-on-disc tribometer, CSM HT 3-110 (CSM Instruments, United States). The ball was immersed in fully formulated oil Shell NS-3, held and loaded by the holder. The disc was heated by the base and rotated at a predefined speed. The tribological data, including lateral force and coefficient of friction, were monitored and recorded by the sensors.

The steel ball was $10 \mathrm{~mm}$ in diameter and made of AISI 52100 with a hardness of 6266 HRC. The chemical compositions are listed in Tab. 1. The centre-line average 
roughness $R_{a}$ is $130 \mathrm{~nm}$. The disc is of the same material of the ball (AISI 52100), with a centre-line average roughness $R_{a}$ of $20 \mathrm{~nm}$, see Fig. 1.

Tab. 1. Chemical composition of AISI 52100.

\begin{tabular}{cc}
\hline Element & Content (\%) \\
\hline $\mathrm{C}$ & $0.980-1.10$ \\
$\mathrm{Cr}$ & $1.30-1.60$ \\
$\mathrm{Fe}$ & $96.5-97.32$ \\
$\mathrm{Mn}$ & $0.250-0.450$ \\
$\mathrm{P}$ & $\leq 0.0250$ \\
$\mathrm{~S}$ & $\leq 0.0250$ \\
$\mathrm{Si}$ & $0.150-0.300$ \\
\hline
\end{tabular}

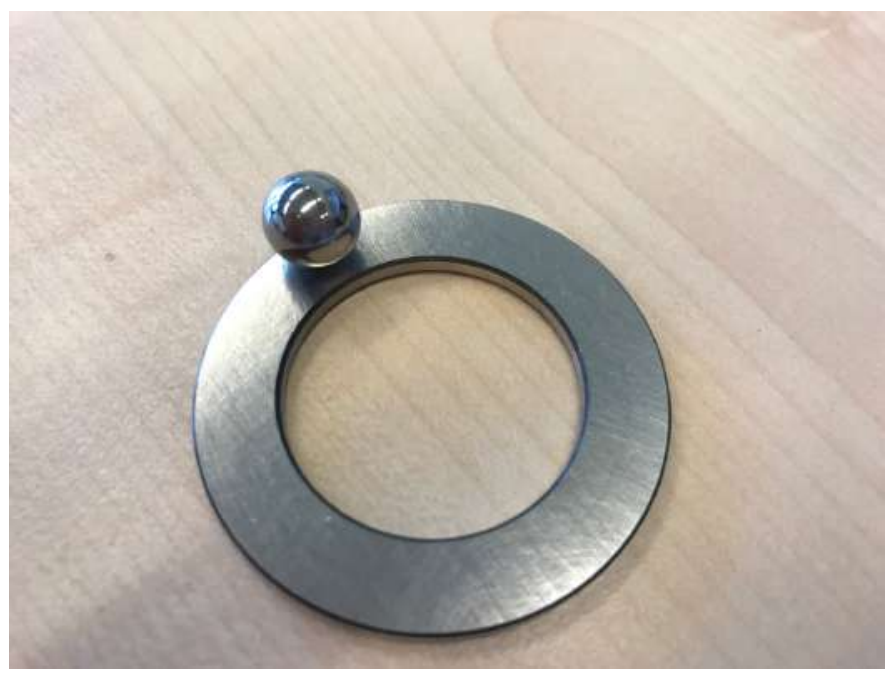

Fig. 1. Ball and disc samples used in the pin-on-disc test.

Both the ball and the disc were ultrasonically cleaned by 2-propanol for $10 \mathrm{~min}$ prior to the test. After the ball and disc were assembled in the test rig, $10 \mathrm{~mL}$ lubricant was applied and the temperature was raised to the set value without applying any load. When the system reached the test temperature, the normal load was then 
applied and the test started. Friction forces were recorded for 12 hours, while the initial running-in periods were excluded. The detailed tests conditions are listed in Tab. 2.

Tab. 2. Running conditions of the pin-on-disc test.

\begin{tabular}{cc}
\hline Parameter & Value \\
\hline Load $(\mathrm{N})$ & 10 \\
Max. Hertzian pressure $(\mathrm{GPa})$ & 1.01 \\
Avg. Hertzian pressure $(\mathrm{GPa})$ & 0.67 \\
Hertzian contact diameter $(\mu \mathrm{m})$ & 138 \\
Sliding velocity $(\mathrm{m} / \mathrm{s})$ & 0.1 \\
Oil temperature $\left({ }^{\circ} \mathrm{C}\right)$ & 80 to 150 \\
Duration $(\mathrm{h})$ & 12 \\
\hline
\end{tabular}

The lateral forces and coefficient of friction data were recorded automatically during the test by the friction sensor at a rate of $5 \mathrm{~Hz}$. The tests were repeated three times for each condition.

\subsection{Post-test surface characterizations}

An optical microscope was used to measure the size of the wear scars. AFM, FIB-SEM and X-ray photoelectron spectroscopy were employed to characterize the morphology, thickness and chemical compositions of the tribofilm respectively. Prior to the surface analysis, the samples were ultrasonically cleaned by 2-propanol for 10 $\mathrm{min}$ in order to remove the residual lubricant and wear debris. The samples were further heated to $60{ }^{\circ} \mathrm{C}$ in a vacuum oven for $30 \mathrm{~min}$ to dissipate the residual detergent of the surfaces.

A Park NX-10 AFM (Park Systems, South Korea) was used to investigate the tribofilm morphology. For the morphology measurement, the AFM ran under the tapping mode, equipped with a beam-shaped, non-contact cantilever (PPP-NCHR) with a nominal resonance frequency of $330 \mathrm{kHz}$. The central parts of the wear scar on the post-test surface were scanned over a $50 * 50 \mu \mathrm{m}^{2}$ area at a scanning rate of $10 \mu \mathrm{m} / \mathrm{s}$. The z-height data in the scanning area were collected at a resolution of $256 * 256$ pixels. 
A Nova 600 Nanolab Dualbeam SEM/FIB (FEI, United States) was used to observe the thicknesses of the tribofilm. For each sample, four $3^{*} 20 \mu \mathrm{m}^{2}$ areas of the surface were milled using a gallium ion beam. The depth of the sputtered area was about 4 $\mu \mathrm{m}$. This process revealed the cross-section profile of the tribofilm and the substrate material beneath. The micrograph of the cross-sections of the film (and substrate) were then recorded by a secondary electron detector located at an angle of $52^{\circ}$ to the direction vertical to the surface of the sample.

The chemical composition of the wear scars were studied by an X-ray photoelectron spectroscopy PHI Quantera SXM (Physical Electronics, United States), with a monochromatic Al Ka X-ray (1486.6 eV) source. The size of the X-ray beam was 100 $\mu \mathrm{m}$. The chemical bonding conditions of the tribofilm were analyzed by detecting the kinetic energy of emitted photoelectrons. The measured spectra were shifted with respect to aliphatic carbon $\mathrm{C} 1 \mathrm{~s}$ at $284.8 \mathrm{eV}$. During the measurement, the residual pressure remained below $5 * 10^{-7} \mathrm{~Pa}$. Survey scans were firstly carried out between 50 and $1100 \mathrm{eV}$ binding energy under a $25 \mathrm{~W}$ constant-analyzer-energy mode, to identify the elements present. The survey scans were performed in three cycles. Next, narrow scans of the selected elements together with the etching technique were conducted in order to identify the chemical compositions in depth profile. For each measuring step, the sample was sputtered with a $3 \mathrm{kV}, 3 * 3 \mathrm{~mm}^{2}$ argon ion beam, removing around $14.5 \mathrm{~nm}$ of material, after which narrow element scans were carried out. This process was repeated nine times for each sample, adding up to 130 $\mathrm{nm}$ in depth.

\subsection{Nanoscale hardness and elastic modulus by AFM}

The hardness and elastic modulus measurements of the tribofilm were conducted in force spectroscopy mode using Dimension 3100 AFM retrofitted with NanoScope IIIa controller (Bruker, USA). Stiff silicon-made rectangular-shaped cantilevers with polycrystalline diamond-coated tips were used to allow a low-wear testing, namely DT-NCHR from Nanosensors (Switzerland). Prior to and after the nanomechanical testing, height images of the surface topography were collected in contact mode using the same tip at the deflection setpoint of 0.5-1.0 V. Imaging and nanomechanical testing were performed using NanoScope software version 8.15. Cantilever (normal) spring constant values, $\boldsymbol{k}_{\boldsymbol{n}}$, were obtained based on their geometry, using formula [43]: 


$$
k_{n}=\frac{E_{c} w t^{3}}{4 l^{3}}
$$

where $\boldsymbol{E}_{\boldsymbol{c}}$ is Young's modulus of the cantilever, $\boldsymbol{w}$ is width of the cantilever, $\boldsymbol{t}$ is the thickness of the cantilever, and $\boldsymbol{l}$ is the length of the cantilever (measured from the end of the chip to the apex of the tip). We assumed the Young's modulus of the cantilever to be $165 \mathrm{GPa}$ [44][45]. The geometry of the cantilever and the tips were measured by SEM imaging after each experiment by JEOL JSM-7610-LA microscope using a low voltage. The radii of the AFM tips, $\boldsymbol{R}$, were determined by calculating the radius of the circle that coincides with the tip apex profile. The range of the obtained dimensions of several cantilevers used is presented in Tab. $\mathbf{3}$.

Tab. 3. Dimensions of the AFM cantilevers used.

\begin{tabular}{cc}
\hline Parameter & Value $(\mu \mathrm{m})$ \\
\hline Length $(\boldsymbol{l})$ & $116-120$ \\
Width $(\boldsymbol{w})$ & $32-42$ \\
Thickness $(\boldsymbol{t})$ & $4-4.3$ \\
Height of the tip $(\boldsymbol{h})$ & $10-11$ \\
Radius of the tip $(\boldsymbol{R})$ & $0.08-0.27$ \\
\hline
\end{tabular}

Thus, based on Eq. (1) $\boldsymbol{k}_{\boldsymbol{n}}$, values ranging from 48.9 - $88.3 \mathrm{~N} / \mathrm{m}$ were obtained. The (normal) sensitivity of the AFM optical system, $\boldsymbol{S}_{\boldsymbol{n}}$, was measured on a quartz reference, fitting the extended part of the force-distance curve.

The NanoScope Analysis software version 1.8 was used to process and evaluate plastic (hardness by indentation) and elastic (Young's modulus by elastic sample response) events obtained by AFM tip-to-surface loading under different normal forces, $\boldsymbol{F}_{\boldsymbol{n}}$. In the case of the hardness calculation (average), around 30 indentations were performed at different spots on the specific sample. The surface topography was imaged before and after to inspect the indentation hole. The projected area of the remaining deformation (hole), $\boldsymbol{A}_{\boldsymbol{p}}$, was calculated by three-dimensional analysis of the hole profile by means of dedicated software. The $\boldsymbol{A}_{\boldsymbol{p}}$ ranged from 8000 to $33000 \mathrm{~nm}^{2}$. The hardness, $\boldsymbol{H}$, was then calculated using the following formula [46][47]: 


$$
H=\frac{F_{n}}{A_{p}}=\frac{k_{n} S_{n} V_{n}}{A_{p}}
$$

where $\boldsymbol{V}_{\boldsymbol{n}}$ is the photo-potential measured vertically with a position sensitive photo detector (PSPD) that is proportional to the (normal) deflection of the cantilever. The applied $\boldsymbol{F}_{\boldsymbol{n}}$ ranged from 19 to $75 \mu \mathrm{N}$.

The Young's moduli values were obtained by evaluating around 50 force-distance curves collected at different spots on the specific sample. The Derjaguin, Muller and Toporov (DMT) model was employed to quantify the tribofilm elasticity. In this model the deformed contact profile is the same as in the Hertz model, however with a higher applied load, $\boldsymbol{F}_{\boldsymbol{D M} \boldsymbol{M}}$, due to an additional adhesion interaction. The following formula was used [48][49]:

$$
F_{D M T}=\frac{4}{3} \frac{E}{(1-v)} \sqrt{R} \delta^{\frac{3}{2}}+F_{a d h}
$$

where $\boldsymbol{E}$ is the Young's modulus of the tested sample, $\boldsymbol{v}$ is the Poisson ratio of the tested sample, $\delta$ is the indentation depth, $\boldsymbol{F}_{\boldsymbol{a d} \boldsymbol{h}}$ is the adhesion force (pull-off force) and $\boldsymbol{F}_{\boldsymbol{n}}=\boldsymbol{F}_{\boldsymbol{D M T}}-\boldsymbol{F}_{\boldsymbol{a d h}}$. We assumed the $\boldsymbol{v}$ to be 0.3 [50]. The $\boldsymbol{F}_{\boldsymbol{n}}, \boldsymbol{\delta}$ and $\boldsymbol{F}_{\boldsymbol{a d h}}$ were calculated directly from the force-distance curves. Due to small indentation depth, up to $8 \mathrm{~nm}$, a "spherical indenter" in the DMT model and a pure elastic material respond could be assumed. The Young's modulus was determined by fitting the slope of the extended part of force-distance curves (fitting parameter). The fitting used a nonlinear least squares fit that goes through the contact point.

\subsection{Lateral force and shear strength by AFM}

The shear strengths of the tribofilm were measured by the Park NX-10 AFM running under LFM mode using the method proposed by Pietrement and Troyon [51]. Data of large scanning areas $\left(50 * 50 \mu \mathrm{m}^{2}\right.$, located in the centre of the wear scars) were 
captured to minimize the effects of local inhomogeneity. The DT-NCHR (Nanosensors) cantilevers were used in this experiment as well.

In LFM mode, the cantilever moves laterally parallel to the surface and it is deflected sideways due to tip-sample interaction (friction). This deflection is recorded by the PSPD as the photo-potential measured laterally, $\boldsymbol{V}_{\boldsymbol{l}}$. The main point of concern - in the calculation of the lateral force and the shear strength - is the determination of the (lateral) sensitivity of the AFM optical system, $\boldsymbol{S}_{\boldsymbol{l}}$, because it cannot be calculated directly from a typical (vertical) force-distance curve [52][53][54] However, when one knows the $\boldsymbol{S}_{\boldsymbol{n}}$, the $\boldsymbol{S}_{\boldsymbol{l}}$ can be calculated from the following formula [52] assuming that the PSPD is rotationally symmetric:

$$
S_{l}=\frac{E_{c}(h+t / 2)}{2 G l} S_{n}
$$

where $\boldsymbol{h}$ is the height of the tip (see Tab. 2) and $\boldsymbol{G}$ is the shear modulus of the cantilever.

The lateral force, $\boldsymbol{F}_{l}$, can then be calculated using the formula:

$$
F_{l}=k_{l} S_{l} V_{l}
$$

where $\boldsymbol{k}_{\boldsymbol{l}}$ is the cantilever (torsional) spring constant given by:

$$
k_{l}=\frac{G w t^{3}}{3 l(h+t / 2)^{2}}
$$

Using Eq. (4) and (6), Eq. (5) can then be rewritten as: 


$$
F_{l}=\frac{E_{c} w t^{3}}{6 l^{2}(h+t / 2)} S_{n} V_{l}
$$

The contact area, $\boldsymbol{A}_{\boldsymbol{c}}$, and the mean contact pressure of the cantilever tip and the surface, $\overline{\boldsymbol{p}}$, can be calculated by the Hertzian contact model [55]:

$$
\begin{gathered}
A_{c}=\pi a^{2} \\
\bar{p}=\frac{F_{n}}{\pi a^{2}}
\end{gathered}
$$

where $\boldsymbol{a}$ is the contact radius, which can be expressed as:

$$
a=\sqrt[3]{\frac{3 F_{n} R\left(1-v^{2}\right)}{4 E}}
$$

The shear strength can then be calculated as:

$$
\tau=\frac{F_{l}}{A_{c}}=\frac{1}{\pi a^{2}} * \frac{E_{c} w t^{3}}{6 l^{2}(h+t / 2)} S_{n} V_{l}
$$

\section{Experimental results and discussions}

\subsection{Friction and wear characteristics of the pin-on-disc samples}

The friction and wear of the pin-on-disc sample pair were measured at different temperatures ( 80 to $150{ }^{\circ} \mathrm{C}$, with $10^{\circ} \mathrm{C}$ interval). An example result (at $80^{\circ} \mathrm{C}, 0$ to 1 h) is shown in Fig. 2. A running-in period occurs from 0 to $0.15 \mathrm{~h}$, where the coefficient of friction changes. Afterwards, the system enters the steady-state, 
indicating by the stable coefficient of friction values. In this study, the running-in periods are trimmed and only the coefficient of friction of the steady-state are considered.

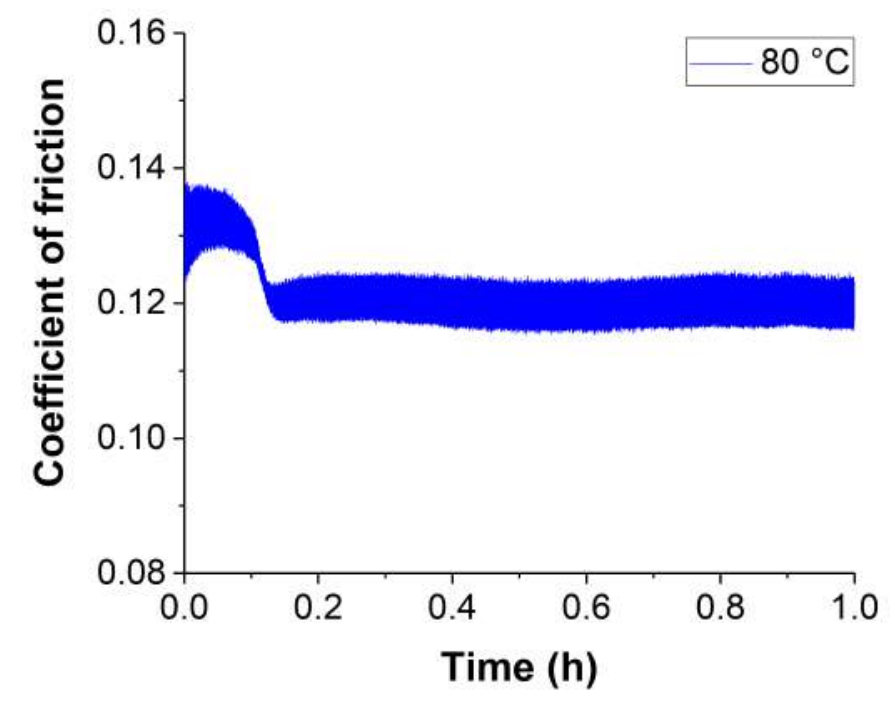

Fig. 2. The running-in and steady-state periods of the test.

The coefficients of friction as a function of temperature are shown in Fig. 3. It is evident that in the temperature range between 80 and $150{ }^{\circ} \mathrm{C}$, the coefficient of friction increases with increasing temperature. At $80^{\circ} \mathrm{C}$, a COF of 0.12 is observed. The COF rises smoothly with temperature. While at $150{ }^{\circ} \mathrm{C}$, the COF is 0.14 , more than $15 \%$ higher than at $80^{\circ} \mathrm{C}$. 


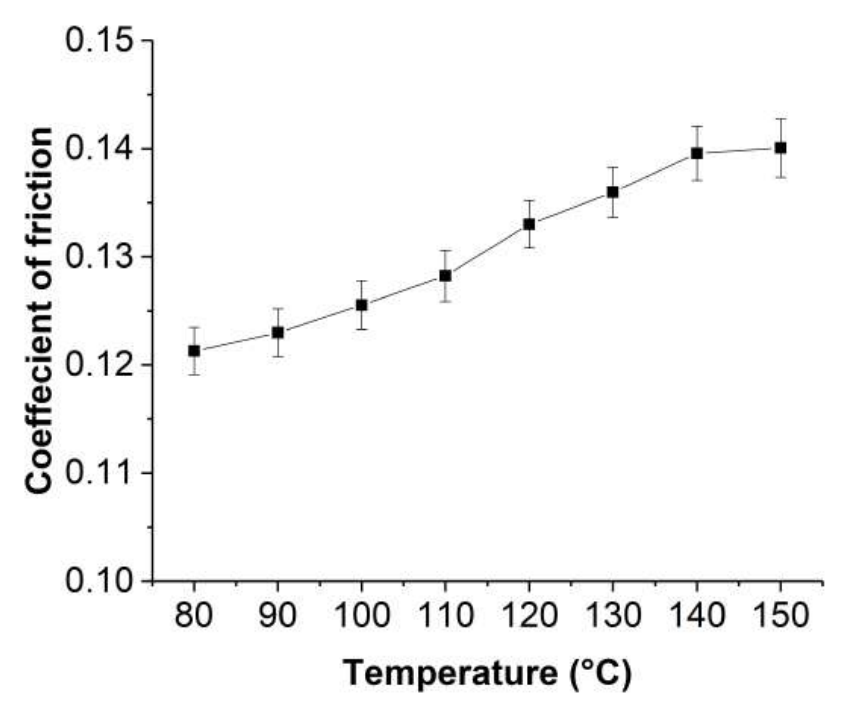

Fig. 3. The coefficient of friction at different temperatures.

The wear of the samples also increases with temperature. The optical microscope images of the wear scars on ball samples tested at 80 and $150^{\circ} \mathrm{C}$ are shown in Fig. 4. For the ball tested at $80^{\circ} \mathrm{C}$, a wear scar of around $200 \mu \mathrm{m}$ diameter is observed, with a wear volume of around $2.51 * 10^{-13} \mathrm{~m}^{3}$. When tested at $150^{\circ} \mathrm{C}$, however, more wear occurs: the diameter of the wear scar significantly increases to around $300 \mu \mathrm{m}$, and the wear volume is around $1.27 * 10^{-12} \mathrm{~m}^{3}$.

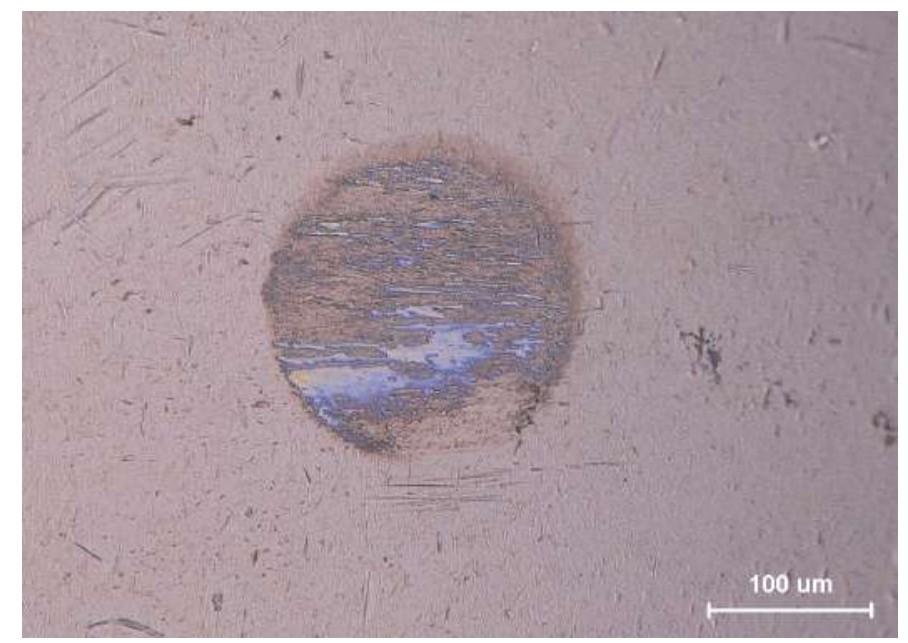

(a) 


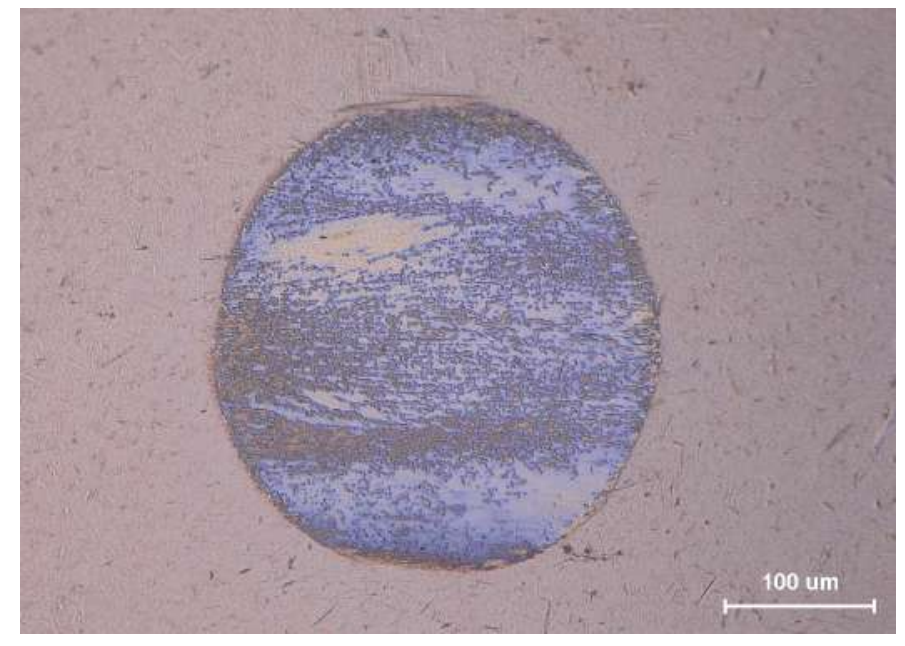

(b)

Fig. 4. Optical images of the wear scars: (a) $80^{\circ} \mathrm{C}$ and (b) $150^{\circ} \mathrm{C}$.

The differences in friction and wear at different temperatures suggest that the temperature plays a role in this tribological process. The reason may be attributed to the fact that the tribofilms formed at different temperatures differ in morphology, thickness, chemical contents and mechanical properties. To better understand this tribological process, further investigation on the tribofilms was carried out and is presented in the following subsections.

\subsection{Morphology, thickness and chemical composition of the tribofilm}

To better understand the origin of the different tribological performances, the tribofilms formed on the wear scars were studied by AFM, SEM-FIB and XPS.

Representative z-height AFM images of post-test ball samples are shown in Fig. $\mathbf{5}$. The rubbing direction is along the y axis. The corresponding cross-section profiles taken perpendicular to the sliding direction - are also presented for comparison. 

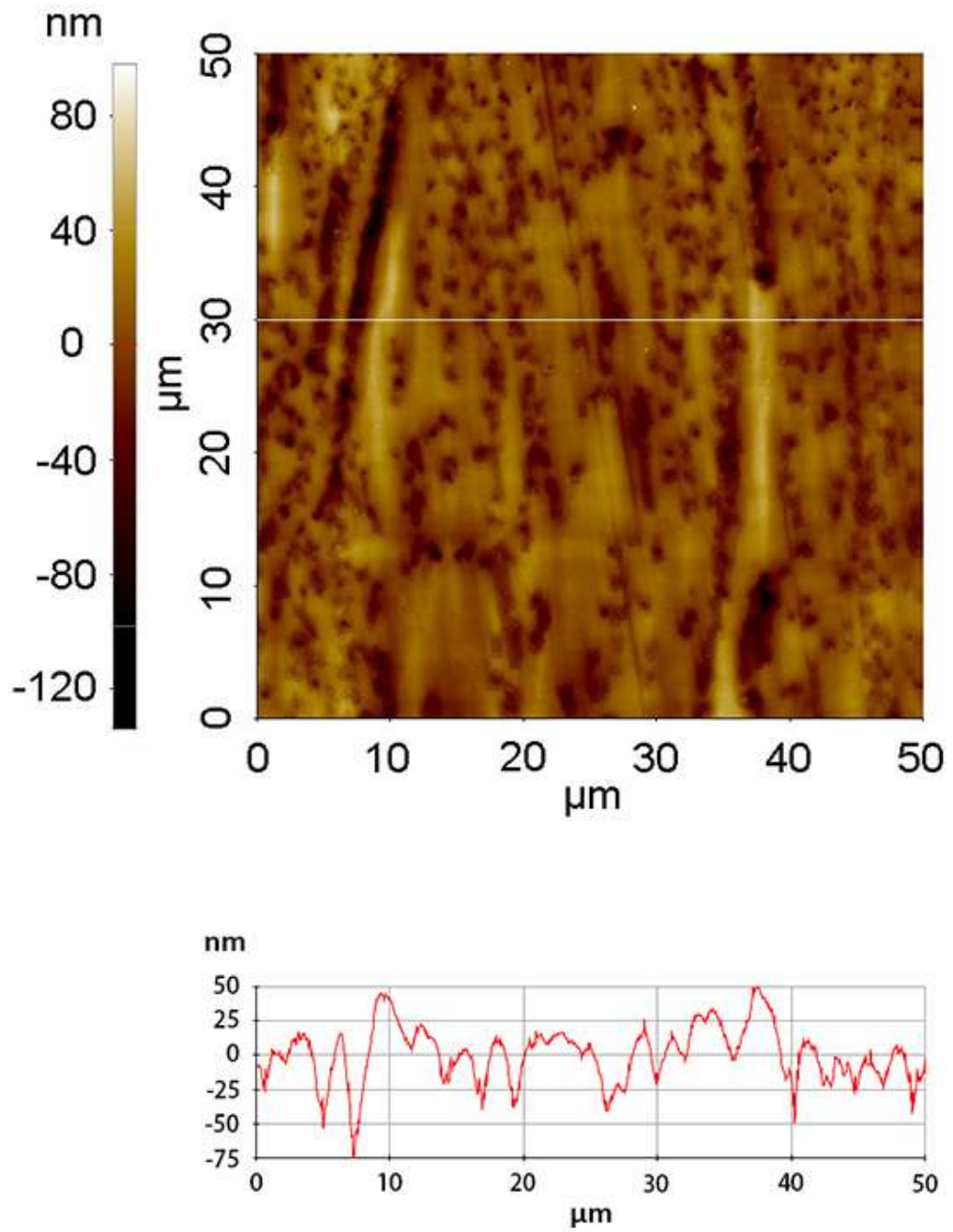

(a) 

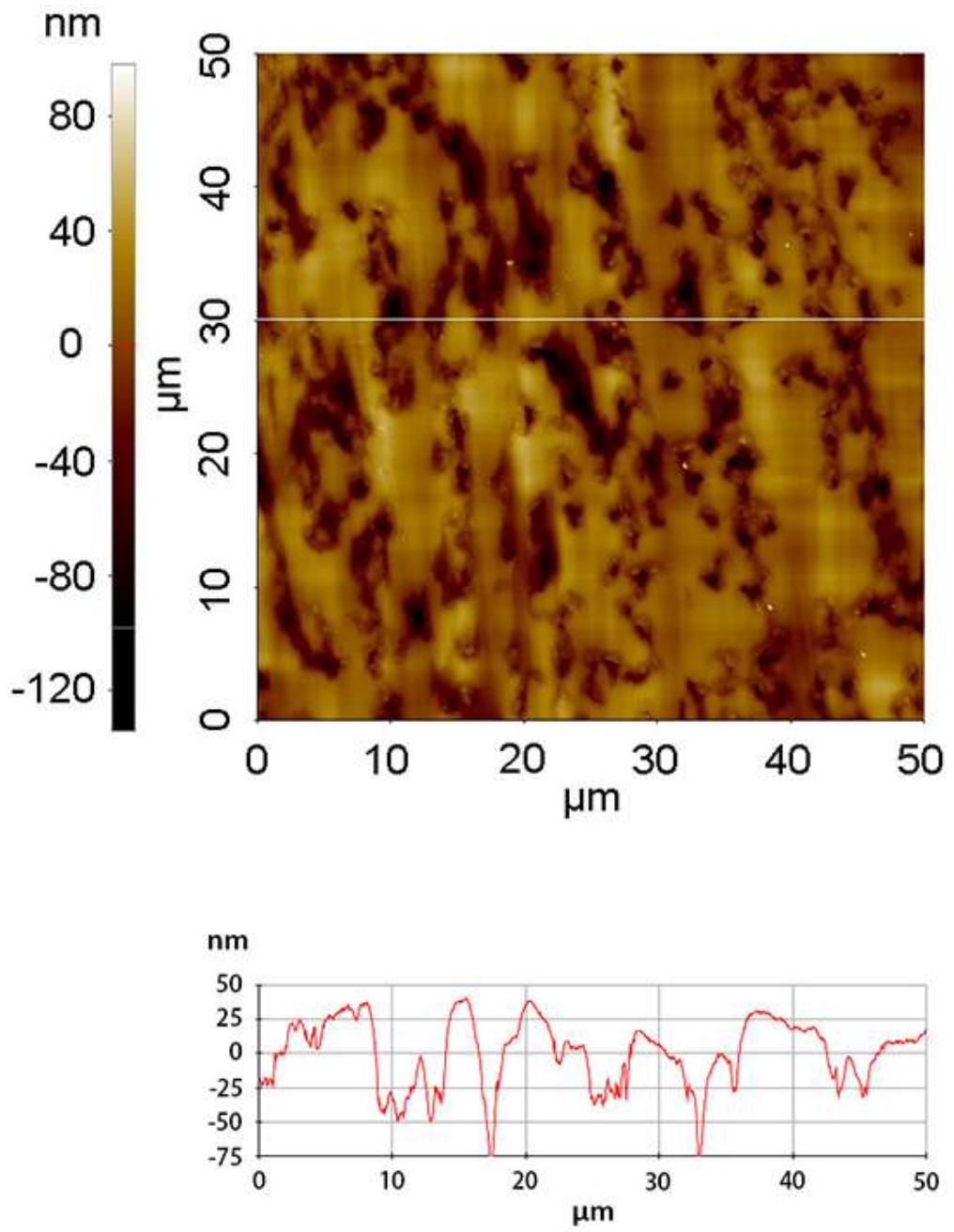

(b)

Fig. 5. AFM height images (tapping mode) and topography profiles (along the section lines) of the tribofilms formed at: (a) $80^{\circ} \mathrm{C}$ and (b) $150{ }^{\circ} \mathrm{C}$.

A similarity can be identified in the morphology of the tribofilms formed at different temperatures. The post-test surfaces are inhomogeneous and pad-like structures are observed along the sliding direction. The bright, elevated pads are tribofilms and the dark valleys are due to the steel substrate not being covered (this is further confirmed by the SEM-FIB images below). Tribofilm pads with the size of 10-20 $\mu \mathrm{m}$ form and elongate in the sliding direction, while tribofilm pads with the size of 1-5 
$\mu \mathrm{m}$ are also observed between the substrate valleys. For both samples, the height differences between peaks and valleys are around 100 to $125 \mathrm{~nm}$, which provide hints of the tribofilm thicknesses. The reason for the minor differences in the tribofilm morphology formed at 80 and at $150^{\circ} \mathrm{C}$ is that larger and more continuous tribofilm pads are formed on the wear scar at $150{ }^{\circ} \mathrm{C}$ than at $80{ }^{\circ} \mathrm{C}$. This can be identified by both the z-height images and the cross-section profiles.

A focused ion beam microscope was used to generate images of the cross-section of the tribofilm. Representative SEM images are presented in Fig. $\mathbf{6}$. It can be identified that the morphology and thickness of the tribofilms formed at different temperatures are rather similar. Most of the surfaces are covered with a darkcoloured tribofilm, a small ratio of the surfaces are steel substrate without tribofilm. This is in good agreement with the AFM topography results. The thicknesses of the tribofilms are measured and labelled in Fig. 6 . For both samples, the thicknesses of the tribofilms are around 120 to $135 \mathrm{~nm}$. No significant differences are observed.

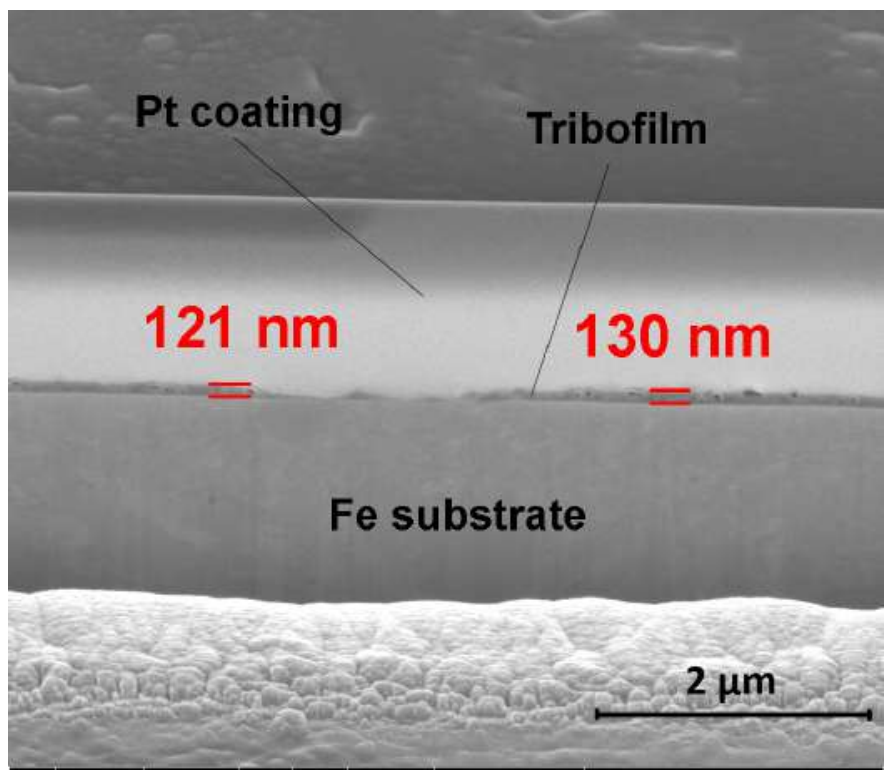

(a) 


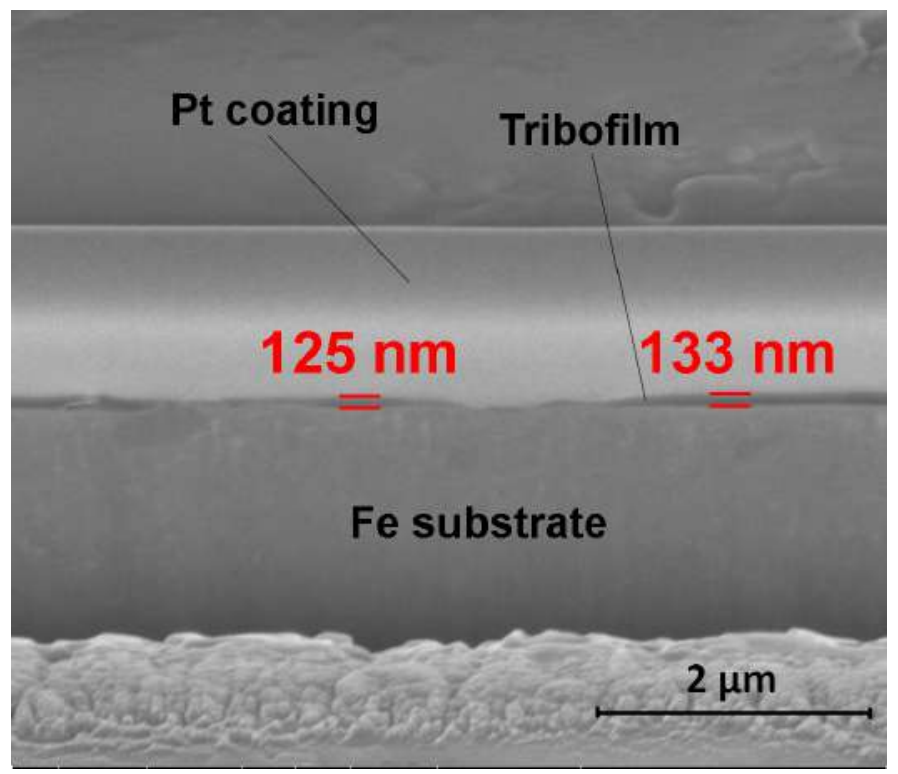

(b)

Fig. 6. FIB-SEM images of the tribofilms: (a) $80^{\circ} \mathrm{C}$ and (b) $150^{\circ} \mathrm{C}$.

The results of the z-height AFM measurements and the SEM-FIB images indicate the continuity of the tribofilms for the samples tested at both 80 and $150^{\circ} \mathrm{C}$. The surfaces are well covered by tribofilms, which prevents direct metal to metal contact. In addition, there is little variation between the thickness of the tribofilms formed at different temperatures. Therefore, the morphology and thickness of the tribofilms are not likely to be the main reason for the different tribological performances. Other factors should be considered.

XPS was employed to analyze the chemical composition of the tribofilms on posttest surfaces. The XPS depth profiling of the tribofilms on the post-test ball samples is shown in Fig. $\mathbf{7}$ and Fig. 8. In the phosphorus spectrum of the samples tested at $80{ }^{\circ} \mathrm{C}$, a sharp peak at $133.0 \mathrm{eV}$ is observed, which corresponds to iron pyrophosphate $\left(\mathrm{Fe}_{4}\left(\mathrm{P}_{2} \mathrm{O}_{7}\right)_{3}\right)$. Therefore, the tribofilm generated under this test condition $\left(80^{\circ} \mathrm{C}\right)$ is composed mainly of short chain iron phosphate. In the calcium spectrum, a sharp peak at $347.2 \mathrm{eV}$ is found. This peak corresponds to the calcium carbonate $\left(\mathrm{CaCO}_{3}\right)$ and calcium oxide $(\mathrm{CaO})$. 


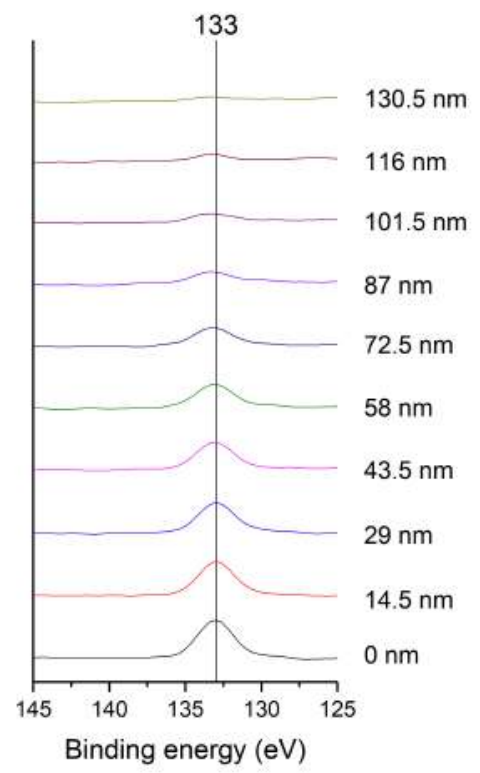

(a)

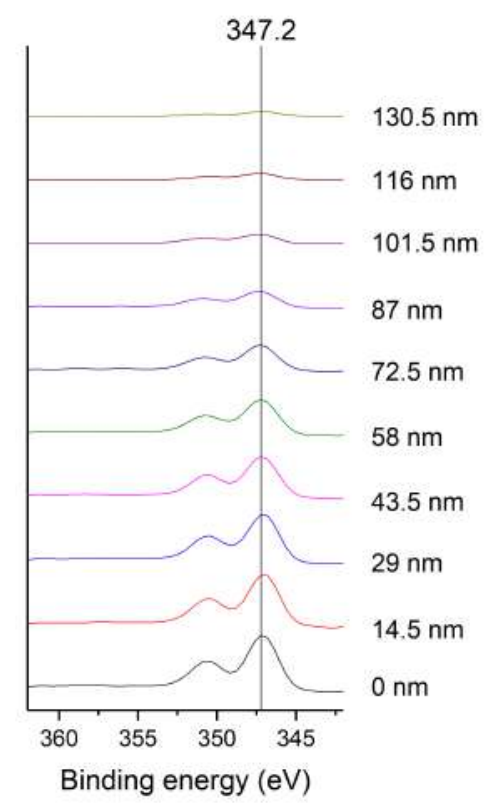

(b)

Fig. 7. XPS spectra of the tribofilm tested at $80^{\circ} \mathrm{C}$ : (a) phosphorus and (b) calcium.

For the $150{ }^{\circ} \mathrm{C}$ case, the XPS depth profiles are shown in Fig. 8. The phosphorus spectrums observed are similar to those for the $80^{\circ} \mathrm{C}$ case: the peaks appear at 133.0 $\mathrm{eV}$, which corresponds to $\mathrm{Fe}_{4}\left(\mathrm{P}_{2} \mathrm{O}_{7}\right)_{3}$. Therefore, it is evident that the similar phosphorus compound is generated at the higher temperature. In the calcium spectrum, however, the peaks occur at $346.8 \mathrm{eV}$ instead of $347.2 \mathrm{eV}$ in the $80^{\circ} \mathrm{C}$ case. This suggests the existence of more $\mathrm{CaO}$ alongside of $\mathrm{CaCO}_{3}$. 


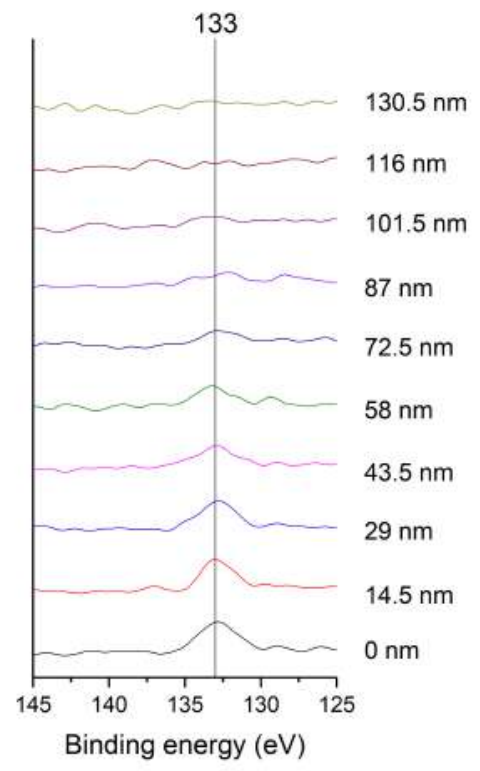

(a)

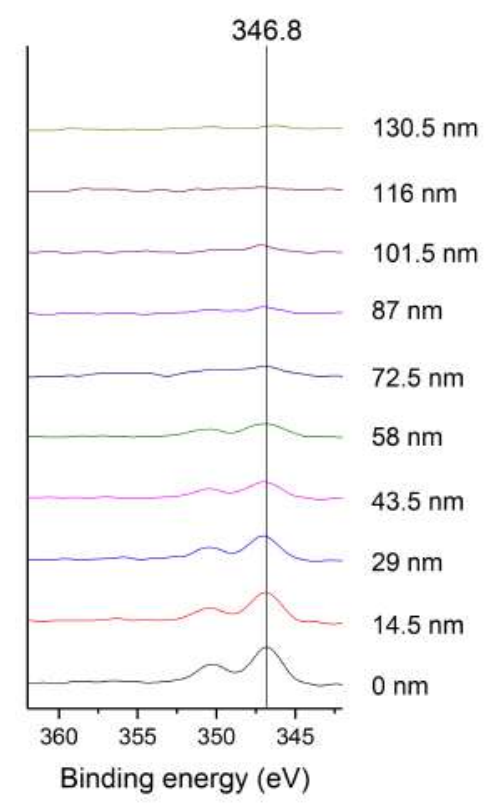

(b)

Fig. 8. XPS spectra of the tribofilm tested at $150^{\circ} \mathrm{C}$ : (a) phosphorus and (b) calcium.

More information about the tribofilms generated at different temperatures was revealed by atom concentrations along the depth, see Fig. 9. The atom concentrations follow a similar trend: $\mathrm{P}$ and $\mathrm{Ca}$ decreases with depth and the $\mathrm{Fe}$ concentration increases with depth. At a depth of $125 \mathrm{~nm}, \mathrm{P}$ and Ca almost vanish in both cases, while the Fe concentrations go beyond $80 \%$. This suggests the tribofilm thickness for both $80^{\circ} \mathrm{C}$ and $150{ }^{\circ} \mathrm{C}$ is around $130 \mathrm{~nm}$, which is in good agreement with the AFM and FIB-SEM results. However, the element concentrations vary for the two samples tested at different temperatures. It can be seen that at the surface the tribofilm generated at $80^{\circ} \mathrm{C}$ contains around $12.5 \%$ of $\mathrm{Fe}$, and almost $40 \%$ in the tribofilm generated at $150^{\circ} \mathrm{C}$. The concentrations of $\mathrm{P}$ and $\mathrm{Ca}$ are lower in the $150^{\circ} \mathrm{C}$ case (both around $5 \%$ ), in contrast to the $80^{\circ} \mathrm{C}$ case (over $10 \%$ ). This clearly suggests that even though the chemical compositions of the tribofilms generated at $80^{\circ} \mathrm{C}$ and $150^{\circ} \mathrm{C}$ are comparable, the concentrations of the elements are rather different. The tribofilm generated at $150^{\circ} \mathrm{C}$ is more Fe rich than that generated at $80^{\circ} \mathrm{C}$. This will 
probably lead to different mechanical properties of the tribofilm and further affect the friction and wear performances.

The mechanical properties of the tribofilms are investigated in the following subsections.

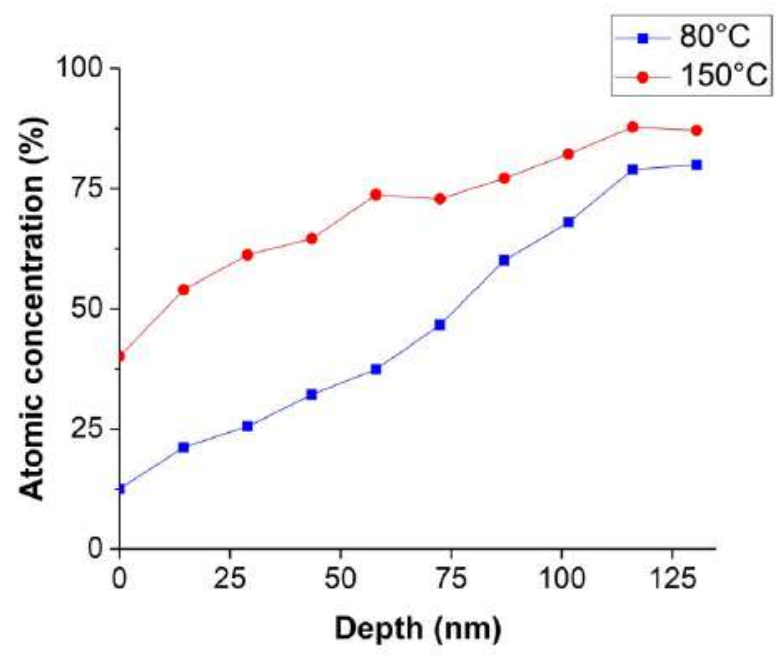

(a)

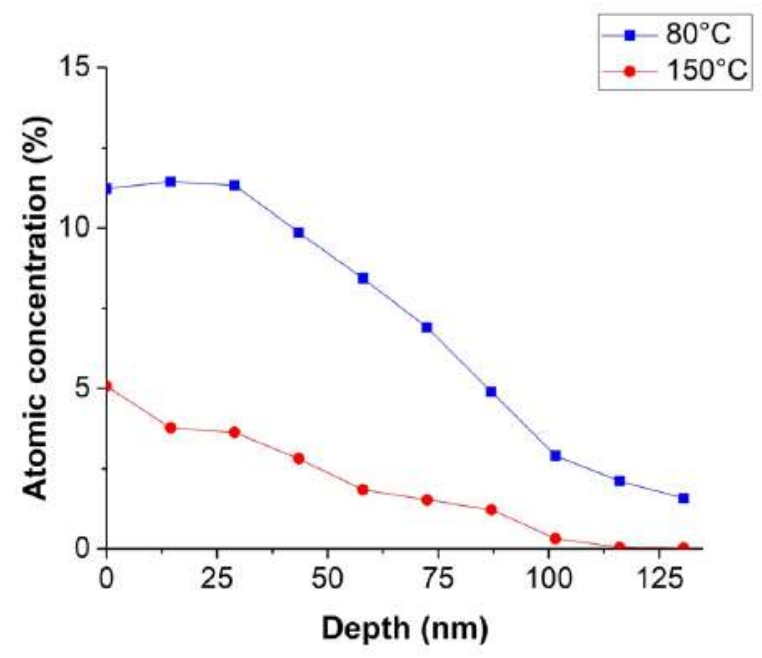

(b) 


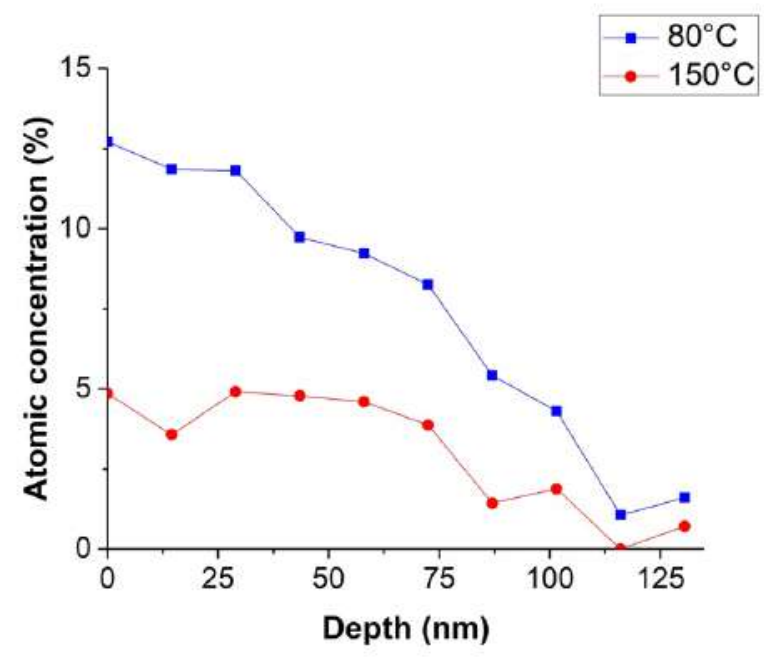

(c)

Fig. 9. XPS depth profile of atomic concentrations: (a) Fe, (b) Ca and (c) P.

3.3. Hardness and Young's modulus of the tribofilm

Fig. 10 represents an example of submicron scale visualization (AFM, contact mode) of tribofilm surfaces with residual plastic impression after an AFM-tip indentation (dark area in the images). Due to the diamond AFM-tip coating the geometry of the indenter varies from one cantilever to another, resulting in differences in the shape of the indentation profile (projected area). The tribofilm formed at $150^{\circ} \mathrm{C}$ turned out to be of a high hardness; high normal loads needed to be applied to obtain a decent indentation with the used AFM setup. The calculated average nanoindentation hardness, $\boldsymbol{H}$, is $4.7 \pm 1.1 \mathrm{GPa}$ for this sample. Differently, for the tribofilm formed at $80{ }^{\circ} \mathrm{C}$ the calculated average $\boldsymbol{H}$ is $2.7 \pm 0.7 \mathrm{GPa}$; this sample can evidently be deformed under lower normal loads. These values fall into similar ranges as those of the hardness of ZDDP tribofilms in [56][57]. 


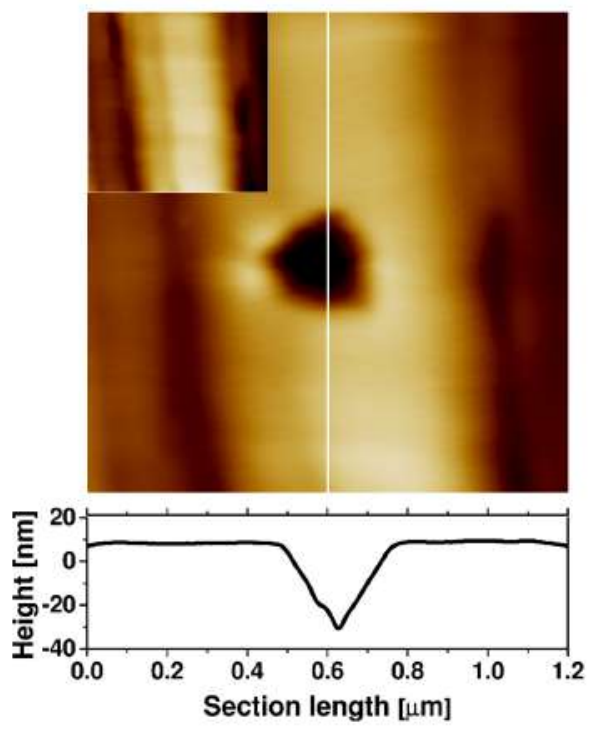

(a)

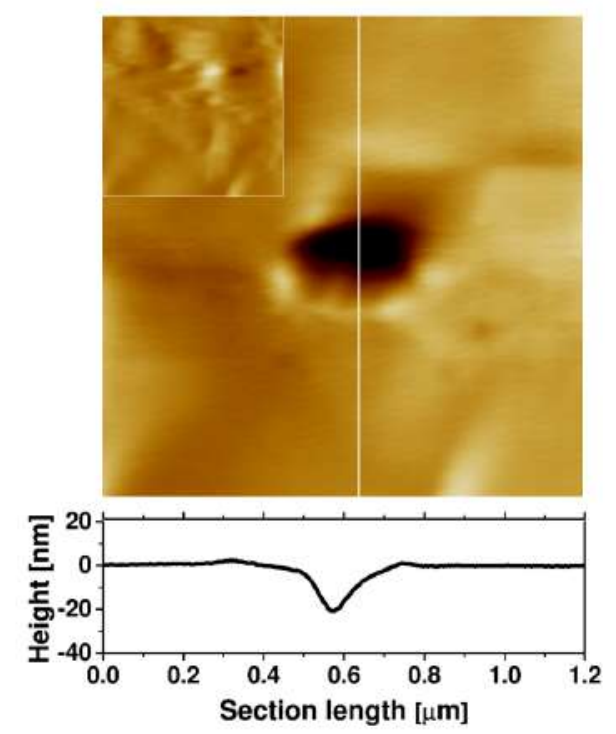

(b)

Fig. 10. AFM height images (contact mode) of the post-indented tribofilm surfaces tested at normal loads of (a) $52 \mu \mathrm{N}$ (film formed at $80^{\circ} \mathrm{C}$ ) and (b) $74 \mu \mathrm{N}$ (film formed at $150{ }^{\circ} \mathrm{C}$ ). The insets represent the investigated area before indentation. Cross-section are plotted along the section lines.

The values of the Young's modulus follow the trend of the hardness result. For the tribofilm formed at $150{ }^{\circ} \mathrm{C}$ the average $\boldsymbol{E}$ is $75 \pm 16 \mathrm{GPa}$, while for the tribofilm formed at $80^{\circ} \mathrm{C}$ it is $56 \pm 14 \mathrm{GPa}$. These values are similar to the Young's modulus of ZDDP tribofilms reported in literature [42][43]. The $\boldsymbol{E}$ value distributions are shown in Fig. 11. 


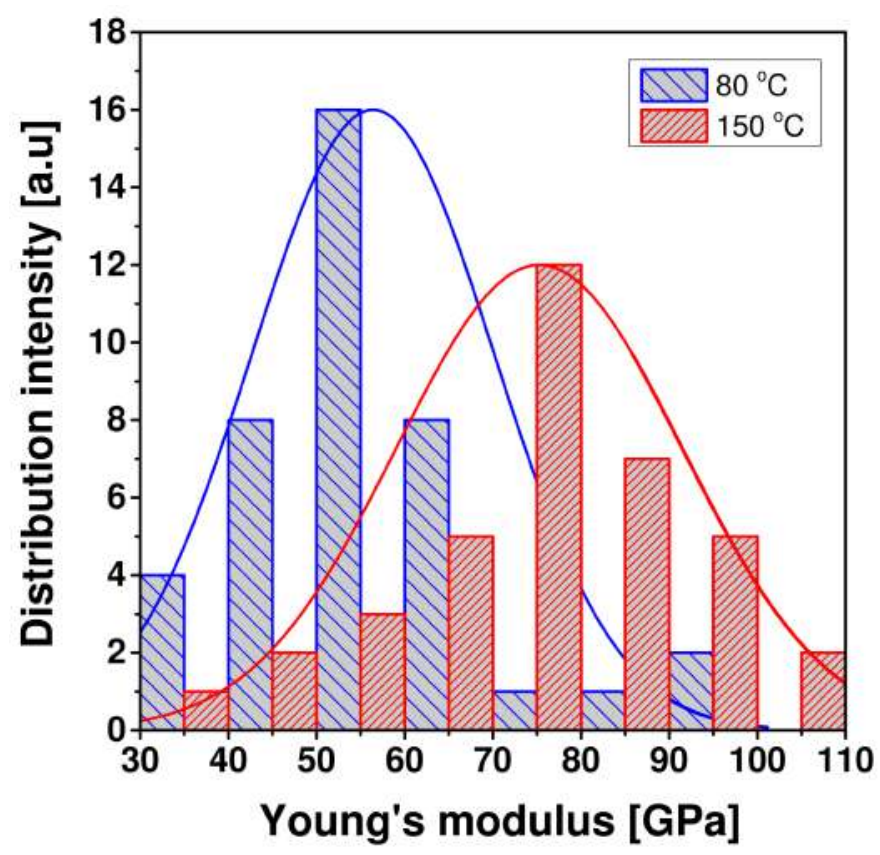

Fig. 11. Young's modulus values histogram for tribofilms formed at 80 and $150{ }^{\circ} \mathrm{C}$. The curves (fit of the normal curve to the distribution) are to guide the eye.

Note that we assumed that the Poisson's ratio of the tribofilm is 0.3 . The results of the elastic modulus results are dependent on the Poisson's ratio being taken, see Eq. (3). Potential errors could arise from this assumption: if the real Poisson's ratio of the tribofilms is in the range of 0.2 to 0.4 , an error range of $86 \%$ to $114 \%$ will be caused in comparison with the value reported.

\subsection{Lateral force and shear strength of the tribofilm}

In the lateral force measurements, the interfacial shearing force of the tribofilms was measured for the further calculations of the shear strength. Three different normal loads $\boldsymbol{F}_{\boldsymbol{n}}$, were applied: $1 \mu \mathrm{N}, 2 \mu \mathrm{N}$ and $5 \mu \mathrm{N}$. These loads were chosen so as to ensure that the top surface of the tribofilm was effectively "sheared" during the measurement instead of just elastically deformed. In addition, the shear forces were measured at three different sliding velocities: $0.01 \mathrm{~mm} / \mathrm{s}, 0.05 \mathrm{~mm} / \mathrm{s}$ and $0.25 \mathrm{~mm} / \mathrm{s}$. 
To illustrate the shearing of the surface, the topography of the tribofilm before and after a lateral force measurement is shown in Fig. $\mathbf{1 2}$ for comparison. The inner area was first tested by the AFM under LFM mode with $2 \mu \mathrm{N}$ normal load. Then the topography of the larger area was imaged for comparison under the tapping mode. It can be seen that the topography of the tribofilm measured by the LFM mode is clearly altered, indicating the occurrence of shear deformation.

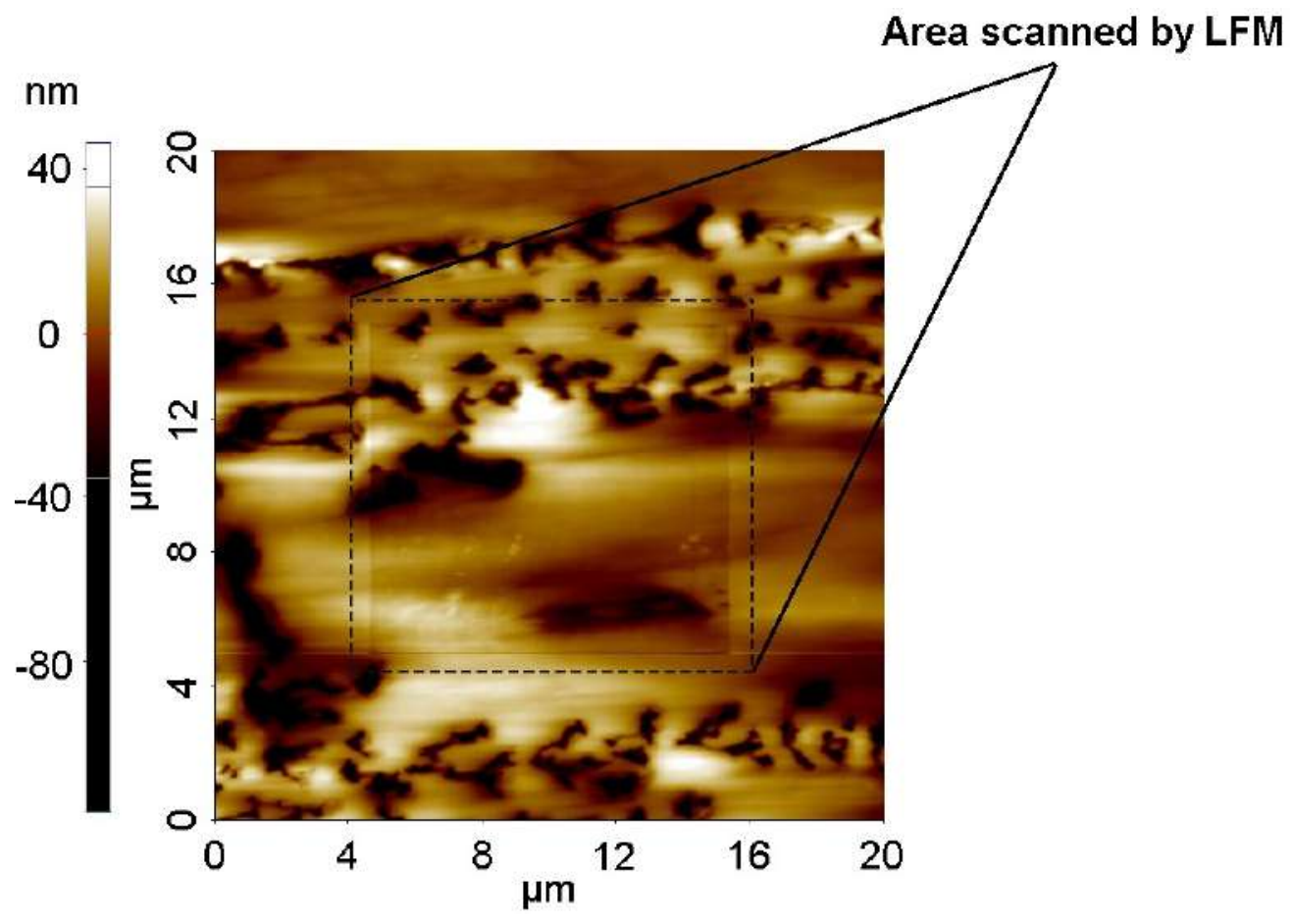

Fig. 12. AFM height image (sample tested at $150^{\circ} \mathrm{C}$ ) obtained after the LFM experiment. The square-marked area indicates the surface of shear deformation under a normal load of $2 \mu \mathrm{N}$.

The raw data of the lateral force measurements are electrical signals with the unit of volts. They are equal to $V_{l}$, representing the lateral torsion. An example image is presented in Fig. 13b. The z-height image of the sample acquired at the same time is also presented for comparison. 


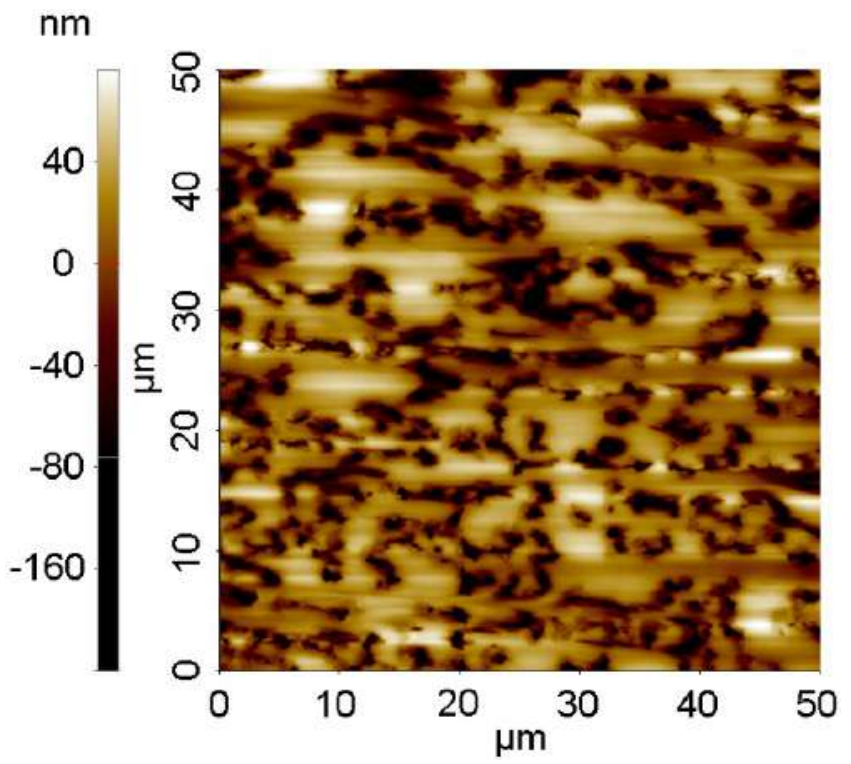

(a)

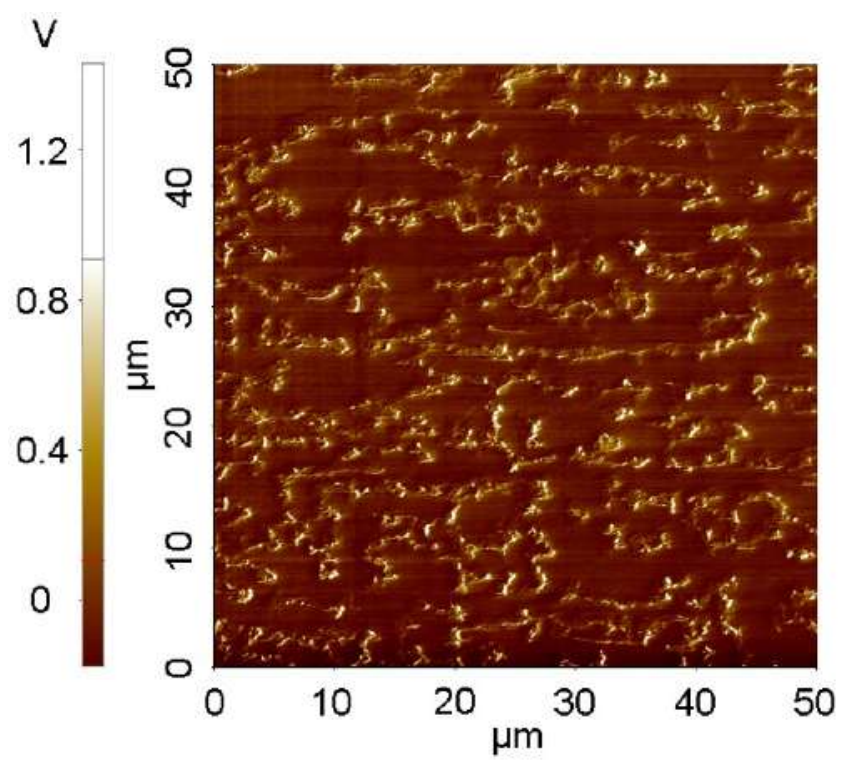

(b)

Fig. 13. AFM results of lateral force measurement (sample tested at $150^{\circ} \mathrm{C}$ ). The normal load is $5 \mu \mathrm{N}$ and the shearing velocity is $0.25 \mathrm{~mm} / \mathrm{s}$.

(a) height image (tapping mode) and (b) representative PSPD lateral force (LFM) image. 
It can be noticed that the lateral force results show an identical pattern to the zheight result, implying that the lateral forces are influenced by the surface roughness. This is anticipated because when the tip travels through bumps or grooves the tilting angle changes due to the surface tilting. However, in the relative flat areas the signals are rather steady. This indicates that the lateral force on these areas is almost constant and can therefore be interpreted as the force for shearing the surface. A line profile of a trace and retrace lateral forces for $80^{\circ} \mathrm{C}$ and $150^{\circ} \mathrm{C}$ can be seen in Fig. 14.

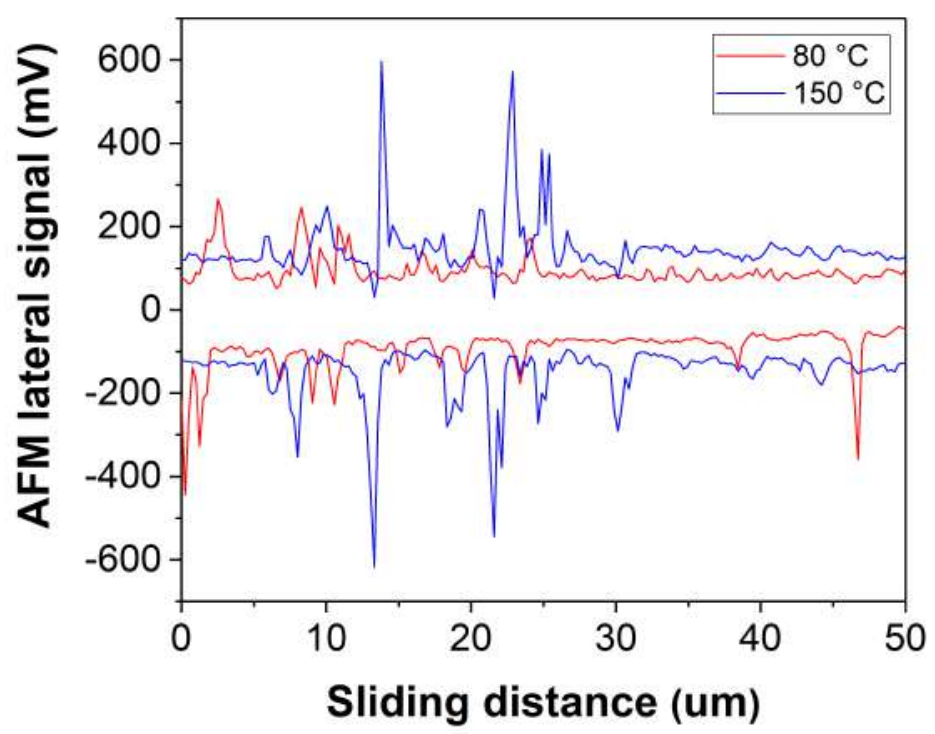

Fig. 14. Line profiles of the lateral force signal. Both cases are measured with $5 \mu \mathrm{N}$ in normal load and $0.25 \mathrm{~mm} / \mathrm{s}$ shearing velocity.

It can be found in Fig. 14 that despite the surface roughness affects the lateral forces, the "base line" of the lateral forces for both the $80^{\circ} \mathrm{C}$ and $150{ }^{\circ} \mathrm{C}$ cases is clearly recognizable. The tribofilm formed at $150{ }^{\circ} \mathrm{C}$ presents a larger lateral force when being sheared, implying a higher shearing force than for the tribofilm formed at $80^{\circ} \mathrm{C}$.

To perform the calculation of the shear strength (see Eq. (11)) the Young's modulus of the tribofilms is taken from the results presented in Subsection 3.3. 
The average results along the scanning areas and the standard deviations are plotted in Fig. 15.

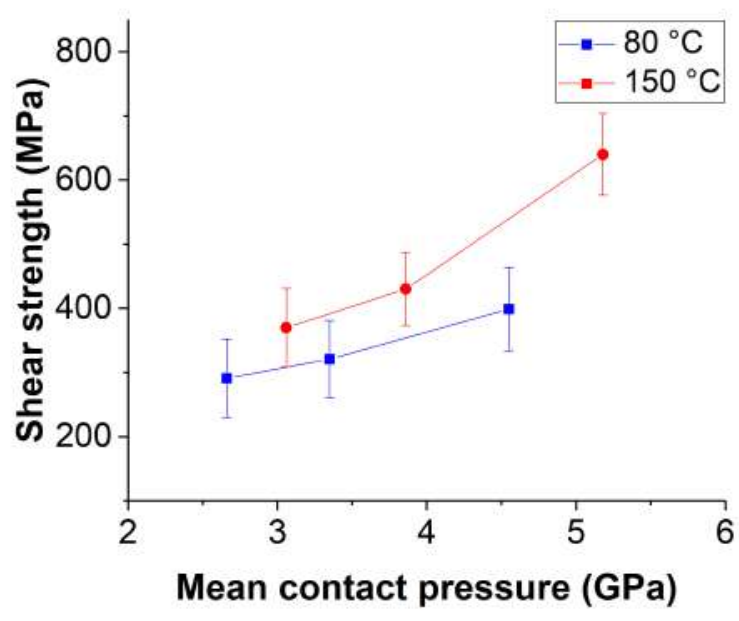

(a)

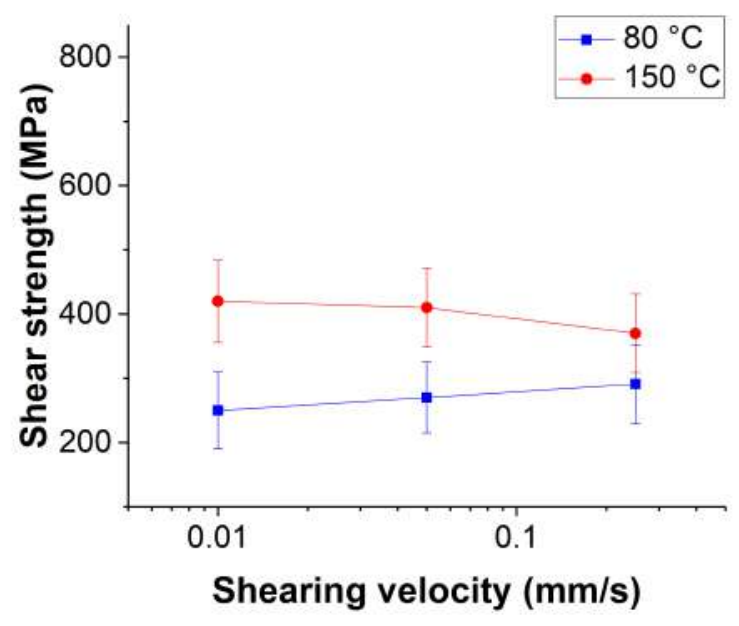

(b)

Fig. 15. AFM shear strength of the tribofilms under different test conditions: (a) Shear strength vs. mean contact pressure (with the shearing velocity of 0.25 $\mathrm{mm} / \mathrm{s}$ ) and (b) Shear strength vs. shearing velocity (with the normal load of $1 \mu \mathrm{N}$ ). 
In general, the results present noticeable standard deviations, which are caused by the surface roughness and the material inhomogeneity. However, the trends are rather clear. The tribofilms formed at $150^{\circ} \mathrm{C}$ show a higher shear strength than the $80^{\circ} \mathrm{C}$ cases under all testing conditions. This is because of the different chemical compositions, which are shown in Subsection 3.2. This higher shear strength may be connected to the higher coefficient of friction observed in Fig. $\mathbf{3}$.

In addition, it can be concluded that the shear strength of the tribofilm is affected by the average contact pressure, but very little affected by the shearing velocity in the range of $0.01 \mathrm{~mm} / \mathrm{s}$ to $0.25 \mathrm{~mm} / \mathrm{s}$.

\section{Discussion}

The results in this work reveal that the frictional and wear properties of the tribosystem are related to the chemical composition and mechanical properties of the tribofilm. The friction and the wear mechanisms are discussed below.

\subsection{Friction mechanisms}

The testing conditions of the LFM for shear strength of the tribofilm are already similar to the real applications $(0.25 \mathrm{~mm} / \mathrm{s}$ shearing velocity and $2.5-5 \mathrm{GPa}$ mean contact pressure, for instance), and the shear strengths acquired in this work can be assumed to be close to reality. The results reveal the correlation between the friction and the shear strength of the tribofilm, as has been suggested in literature.

For the case tested under $0.25 \mathrm{~mm} / \mathrm{s}$ shearing velocity and $\sim 3 \mathrm{GPa}$ mean contact pressure, the shear strength of the tribofilm formed at $150{ }^{\circ} \mathrm{C}$ is $370 \mathrm{MPa}$, while at $80^{\circ} \mathrm{C}$ the shear strength is $291 \mathrm{MPa}, 21 \%$ smaller than the $150^{\circ} \mathrm{C}$ case. This difference is very similar to the difference in the coefficients of friction, which are 0.14 at $150^{\circ} \mathrm{C}$ and 0.12 at $80^{\circ} \mathrm{C}$, i.e. $14.3 \%$ smaller. This similarity supports the equation proposed in literature for the friction in boundary lubrication:

$$
\mathbf{F}=\boldsymbol{\tau} * A_{\text {real }}
$$


Because the post-test surface topography at different temperatures is similar, the real contact areas are comparable. Therefore, the higher shear strength $\boldsymbol{\tau}$ at $150{ }^{\circ} \mathrm{C}$ contributes to the higher friction force and coefficient of friction.

\subsection{Wear mechanisms}

In this work, the testing duration of the pin-on-disc test is 12 hours, which is long enough for the system to reach the steady, mild wear state. During this stage, the main wear mechanism is tribochemical wear, see Fig. 4.

The XPS atomic concentration results show that the Fe content in the tribofilm formed at $150{ }^{\circ} \mathrm{C}$ is richer than that formed at $80{ }^{\circ} \mathrm{C}$. The reason might be that at elevated temperature the chemical reactivity of the Fe with the additive package is higher and that more Fe atoms reacted with the additives, form the tribofilm and are sheared during sliding. This is in line with the fact that the wear volume is higher at $150{ }^{\circ} \mathrm{C}$ than at $80^{\circ} \mathrm{C}$.

\section{Conclusions}

In this work, pin-on-disc sliding tests lubricated by the fully formulated oil Shell NS-3 are carried out. The friction observed is linked to the morphology, thickness, chemical compositions and shear strength of the tribofilm that are measured by a variety of characterizing techniques. The findings are summarized as follows.

(1) The AISI 52100 friction pair lubricated by Shell NS-3 shows different friction levels when running at different temperatures. The coefficient of friction increases when the testing temperatures rise from $80^{\circ} \mathrm{C}$ to $150^{\circ} \mathrm{C}$. The wear scar also increases with higher temperature, indicating a higher wear rate at higher temperature.

(2) The tribofilms formed at $80^{\circ} \mathrm{C}$ and $150^{\circ} \mathrm{C}$ are similar in morphology and thickness but differ in chemical composition. The tribofilm formed at $150^{\circ} \mathrm{C}$ contains higher Fe in all depths than the tribofilm formed at $80{ }^{\circ} \mathrm{C}$. It is assumed that the chemical reactivity of the Fe substrate is higher at elevated temperatures. It leads to higher tribochemical wear rate, which explains the larger wear scar observed at $150{ }^{\circ} \mathrm{C}$.

(3) The tribofilms showed a noticeable difference in hardness and Young's modulus. The film formed at $150{ }^{\circ} \mathrm{C}$ reveals high hardness ( $4.7 \mathrm{GPa}$ ) and Young's modulus ( $75 \mathrm{GPa}$ ). We attribute this to the high Fe content in the tribofilm formed at $150{ }^{\circ} \mathrm{C}$. Differently, the tested nanomechanical properties of film formed at $80^{\circ} \mathrm{C}$ indicate a 
drop in hardness ( $43 \%$ ) and Young's modulus ( 25\%) in comparison with the tribofilm formed at $150{ }^{\circ} \mathrm{C}$.

(4) Different shear strengths of the tribofilms formed at different temperatures are observed. The tribofilm formed at $150{ }^{\circ} \mathrm{C}$ shows higher shear strength than its counterpart at $80^{\circ} \mathrm{C}$. In addition, it is found that the shear strengths of the tribofilm are dependent on the mean contact pressure but are very little affected by the shearing velocity in the range of 0.01 to $0.25 \mathrm{~mm} / \mathrm{s}$.

(5) The different mechanical properties of the tribofilms formed at different temperatures may be linked to the higher Fe content in the tribofilm formed at $150{ }^{\circ} \mathrm{C}$. This could also be the reason for the higher coefficient of friction that is observed in the pin-on-disc test at $150{ }^{\circ} \mathrm{C}$.

\section{Acknowledgement}

The authors would like to express their deepest appreciation to Robert Jan Meijer and Erik G. de Vries for their valuable help and discussions of the LFM tests.

\section{References}

[1] Larsson, P., Axen, N., \& Hogmark, S. (1999). Tribofilm formation on boron carbide in sliding wear. Wear, 236(1-2), 73-80.

[2] Kato, H., \& Komai, K. (2007). Tribofilm formation and mild wear by tribosintering of nanometer-sized oxide particles on rubbing steel surfaces. Wear, 262(1-2), 36-41.

[3] Equey, S., Roos, S., Mueller, U., Hauert, R., Spencer, N. D., \& Crockett, R. (2008). Tribofilm formation from ZnDTP on diamond-like carbon. Wear, 264(3-4), 316321.

[4] Tomala, A., Vengudusamy, B., Ripoll, M. R., Suarez, A. N., Remškar, M., \& Rosentsveig, R. (2015). Interaction between selected MoS 2 nanoparticles and ZDDP tribofilms. Tribology Letters, 59(1), 26.

[5] Aboua, K. A. M., Umehara, N., Kousaka, H., Tokoroyama, T., Murashima, M., Tasdemir, H. A., ... \& Higuchi, T. (2018). Effect of ZnDTP tribofilm's morphology on friction behaviors of DLC coatings: Tribofilm characterization by 3D 
scanning electron microscope observation. Journal of Advanced Mechanical Design, Systems, and Manufacturing, 12(7), JAMDSM0129-JAMDSM0129.

[6] Gosvami, N. N., Lahouij, I., Ma, J., \& Carpick, R. W. (2019). Nanoscale in situ study of ZDDP tribofilm growth at aluminum-based interfaces using atomic force microscopy. Tribology International, 106075.

[7] Zhang, Z., Yamaguchi, E. S., Kasrai, M., \& Bancroft, G. M. (2005). Tribofilms generated from ZDDP and DDP on steel surfaces: Part 1, growth, wear and morphology. Tribology Letters, 19(3), 211-220.

[8] Narita, K., \& Priest, M. (2009). Friction Characteristics and Topography of Tribofilms from Anti-Wear Additives Applied to Metal V-Belt Type CVT Fluids. Tribology Letters, 35(1), 45-56.

[9] Tomala, A., Vengudusamy, B., Ripoll, M. R., Suarez, A. N., Remškar, M., \& Rosentsveig, R. (2015). Interaction between selected MoS 2 nanoparticles and ZDDP tribofilms. Tribology Letters, 59(1), 26.

[10] Shimizu, Y., \& Spikes, H. A. (2016). The influence of slide-roll ratio on ZDDP tribofilm formation. Tribology Letters, 64(2), 19.

[11] Gosvami, N. N., Bares, J. A., Mangolini, F., Konicek, A. R., Yablon, D. G., \& Carpick, R. W. (2015). Mechanisms of antiwear tribofilm growth revealed in situ by single-asperity sliding contacts. Science, 348(6230), 102-106.

[12] Minfray, C., Martin, J. M., Esnouf, C., Le Mogne, T., Kersting, R., \& Hagenhoff, B. (2004). A multi-technique approach of tribofilm characterisation. Thin Solid Films, 447, 272-277.

[13] Ito, K., Martin, J. M., Minfray, C., \& Kato, K. (2006). Low-friction tribofilm formed by the reaction of ZDDP on iron oxide. Tribology International, 39(12), 1538-1544.

[14] Qu, J., Meyer III, H. M., Cai, Z. B., Ma, C., \& Luo, H. (2015). Characterization of ZDDP and ionic liquid tribofilms on non-metallic coatings providing insights of tribofilm formation mechanisms. Wear, 332, 1273-1285.

[15] Dawczyk, J., Ware, E., Ardakani, M., Russo, J., \& Spikes, H. (2018). Use of FIB to study ZDDP tribofilms. Tribology Letters, 66(4), 155.

[16] Duan, Z., Zhao, X., Qiao, L., Zhao, Y., Fu, E., Wang, P., \& Liu, W. (2019). Structural evolution and wear resistance of MoS2-based lubricant films irradiated by heavy ions. Surface and Coatings Technology, 125077.

[17] Morina, A., Green, J. H., Neville, A., \& Priest, M. (2003). Surface and tribological characteristics of tribofilms formed in the boundary lubrication regime with application to internal combustion engines. Tribology Letters, 15(4), 443-452. 
[18] Morina, A., \& Neville, A. (2007). Understanding the composition and low friction tribofilm formation/removal in boundary lubrication. Tribology International, 40(10-12), 1696-1704.

[19] Mourhatch, R., \& Aswath, P. B. (2009). Nanoscale properties of tribofilms formed with zinc dialkyl dithiophosphate (ZDDP) under extreme pressure condition. Journal of Nanoscience and Nanotechnology, 9(4), 2682-2691.

[20] Soltanahmadi, S., Morina, A., van Eijk, M. C., Nedelcu, I., \& Neville, A. (2017). Experimental observation of zinc dialkyl dithiophosphate (ZDDP)-induced iron sulphide formation. Applied Surface Science, 414, 41-51.

[21] Gachot, C., Hsu, C., Suárez, S., Grützmacher, P., Rosenkranz, A., Stratmann, A., \& Jacobs, G. (2016). Microstructural and chemical characterization of the tribolayer formation in highly loaded cylindrical roller thrust bearings. Lubricants, 4(2), 19.

[22] Martin, J. M., Grossiord, C., Le Mogne, T., Bec, S., \& Tonck, A. (2001). The twolayer structure of Zndtp tribofilms: Part I: AES, XPS and XANES analyses. Tribology International, 34(8), 523-530.

[23] De Barros, M. I., Bouchet, J., Raoult, I., Le Mogne, T., Martin, J. M., Kasrai, M., \& Yamada, Y. (2003). Friction reduction by metal sulfides in boundary lubrication studied by XPS and XANES analyses. Wear, 254(9), 863-870.

[24] Ma, H., Li, J., Chen, H., Zuo, G., Yu, Y., Ren, T., \& Zhao, Y. (2009). XPS and XANES characteristics of tribofilms and thermal films generated by two P-and/or Scontaining additives in water-based lubricant. Tribology International, 42(6), 940-945.

[25] Wu, X., Gong, K., Zhao, G., Lou, W., Wang, X., \& Liu, W. (2018). Surface modification of MoS2 nanosheets as effective lubricant additives for reducing friction and wear in poly- $\alpha$-olefin. Industrial \& Engineering Chemistry Research, 57(23), 8105-8114.

[26] Gachot, C., Hsu, C., Suárez, S., Grützmacher, P., Rosenkranz, A., Stratmann, A., \& Jacobs, G. (2016). Microstructural and chemical characterization of the tribolayer formation in highly loaded cylindrical roller thrust bearings. Lubricants, 4(2), 19.

[27] Wu, X., Gong, K., Zhao, G., Lou, W., Wang, X., \& Liu, W. (2018). Surface modification of MoS2 nanosheets as effective lubricant additives for reducing friction and wear in poly- $\alpha$-olefin. Industrial \& Engineering Chemistry Research, 57(23), 8105-8114.

[28] Zhou, Y., Weber, J., Viola, M. B., \& Qu, J. (2019). Is more always better? Tribofilm evolution and tribological behavior impacted by the concentration of ZDDP, ionic liquid, and ZDDP-Ionic liquid combination. Wear, 432, 202951. 
[29] Carpick, R. W., \& Salmeron, M. (1997). Scratching the surface: Fundamental investigations of tribology with atomic force microscopy. Chemical Reviews, 97(4), 1163-1194.

[30] Mo, Y., Turner, K. T., \& Szlufarska, I. (2009). Friction laws at the nanoscale. Nature, 457(7233), 1116.

[31] Stachowiak, G., \& Batchelor, A. W. (2013). Engineering tribology. ButterworthHeinemann.

[32] Mortier, R. M., Orszulik, S. T., \& Fox, M. F. (Eds.). (2010). Chemistry and technology of lubricants (Vol. 107115). New York: Springer.

[33] Tripaldi, G., Vettor, A., \& Spikes, H. (1996). Friction behaviour of ZDDP films in the mixed, boundary/EHD regime. SAE Transactions, 1819-1830.

[34] McQueen, J. S., Gao, H., Black, E. D., Gangopadhyay, A. K., \& Jensen, R. K. (2005). Friction and wear of tribofilms formed by zinc dialkyl dithiophosphate antiwear additive in low viscosity engine oils. Tribology International, 38(3), 289-297.

[35] Green, J. H., Morina, A., Priest, M., \& Neville, A. (2003). Evolution of tribofilms under lubrication conditions experienced in engine valve trains. In Tribology Series (Vol. 43, pp. 97-108). Elsevier.

[36] Morina, A., \& Neville, A. (2007). Tribofilms: aspects of formation, stability and removal. Journal of Physics D: Applied Physics, 40(18), 5476.

[37] Bec, S., Tonck, A., Georges, J. M., \& Roper, G. W. (2004). Synergistic effects of MoDTC and ZDTP on frictional behaviour of tribofilms at the nanometer scale. Tribology Letters, 17(4), 797-809.

[38] Aktary, M., McDermott, M. T., \& McAlpine, G. A. (2002). Morphology and nanomechanical properties of ZDDP antiwear films as a function of tribological contact time. Tribology Letters, 12(3), 155-162.

[39] Pereira, G., Munoz-Paniagua, D., Lachenwitzer, A., Kasrai, M., Norton, P. R., Capehart, T. W., ... \& Cheng, Y. T. (2007). A variable temperature mechanical analysis of ZDDP-derived antiwear films formed on 52100 steel. Wear, 262(34), 461-470.

[40] Ueda, M., Kadiric, A., \& Spikes, H. (2019). On the Crystallinity and Durability of ZDDP Tribofilm. Tribology Letters, 67(4), 123.

[41] Pidduck, A. J., \& Smith, G. C. (1997). Scanning probe microscopy of automotive anti-wear films. Wear, 212(2), 254-264.

[42] Kano, M., Yasuda, Y., \& Ye, J. P. (2004). The effect of ZDDP and MoDTC additives in engine oil on the friction properties of DLC-coated and steel cam followers. Lubrication Science, 17(1), 95-103. 
[43] Wolter, O., Bayer, T., \& Greschner, J. (1991). Micromachined silicon sensors for scanning force microscopy. Journal of Vacuum Science \& Technology B: Microelectronics and Nanometer Structures Processing, Measurement, and Phenomena, 9(2), 1353-1357.

[44] Sadeghian, H., Goosen, H., Bossche, A., Thijsse, B., \& Van Keulen, F. (2011). On the size-dependent elasticity of silicon nanocantilevers: impact of defects. Journal of Physics D: Applied Physics, 44(7), 072001.

[45] Zhang, W. W., Yu, H., Lei, S. Y., \& Huang, Q. A. (2011). Modelling of the elastic properties of crystalline silicon using lattice dynamics. Journal of Physics D: Applied Physics, 44(33), 335401.

[46] Butt, H. J., Cappella, B., \& Kappl, M. (2005). Force measurements with the atomic force microscope: Technique, interpretation and applications. Surface Science Reports, 59(1-6), 1-152.

[47] Fischer-Cripps, A., \& Nicholson, D. (2004). Nanoindentation. Mechanical Engineering Series.

[48] Derjaguin, B. V., Muller, V. M., \& Toporov, Y. P. (1975). Effect of contact deformations on the adhesion of particles. Journal of Colloid and Interface Science, 53(2), 314-326.

[49] Gojzewski, H., Sadej, M., Andrzejewska, E., \& Kokowska, M. (2017). Nanoscale Young's modulus and surface morphology in photocurable polyacrylate/nanosilica composites. European Polymer Journal, 88, 205-220.

[50] Wang, C., \& Schipper, D. (2018). On an elastoplastic sliding model for a coated single asperity. Lubricants, 6(4), 96.

[51] Pietrement, O., \& Troyon, M. (2001). Study of the interfacial shear strength pressure dependence by modulated lateral force microscopy. Langmuir, 17(21), 6540-6546.

[52] Liu, W., Bonin, K., \& Guthold, M. (2007). Easy and direct method for calibrating atomic force microscopy lateral force measurements. Review of Scientific Instruments, 78(6), 063707.

[53] Sader, J. E., Larson, I., Mulvaney, P., \& White, L. R. (1995). Method for the calibration of atomic force microscope cantilevers. Review of Scientific Instruments, 66(7), 3789-3798.

[54] Neumeister, J. M., \& Ducker, W. A. (1994). Lateral, normal, and longitudinal spring constants of atomic force microscopy cantilevers. Review of Scientific Instruments, 65(8), 2527-2531.

[55] Johnson, K. L., \& Johnson, K. L. (1987). Contact mechanics. Cambridge University Press. 
[56] Nicholls, M. A., Norton, P. R., Bancroft, G. M., Kasrai, M., Do, T., Frazer, B. H., \& De Stasio, G. (2004). Nanometer scale chemomechanical characterization of antiwear films. Tribology Letters, 17(2), 205-216.

[57] Nicholls, M. A., Do, T., Norton, P. R., Kasrai, M., \& Bancroft, G. M. (2005). Review of the lubrication of metallic surfaces by zinc dialkyl-dithiophosphates. Tribology International, 38(1), 15-39. 


\section{Paper D}

Wang, C., \& Schipper, D. J. (2021). A sliding dry friction model considering surface plastic deformation and adhesion. To be submitted 



\title{
A sliding dry friction model considering surface plastic deformation and adhesion
}

\author{
Can Wang ${ }^{*}$ and Dik J. Schipper \\ Laboratory for Surface Technology and Tribology, Department of Engineering Technology, \\ University of Twente, 7522 NB Enschede, The Netherlands \\ *Correspondence: c.wang-1@utwente.nl
}

\begin{abstract}
In this paper, a sliding dry friction model was proposed and validated by a pin-ondisc tribo-system under different loads and at different temperatures. Two sources of friction were considered, namely plastic deformation and adhesion. The contribution of plastic deformation was modelled by a semi-analytical method (SAM) based single asperity model, while the adhesion was calculated based on the real contact area and surface shear strengths. The results suggest that for the tribosystem being investigated, adhesion dominates the mechanism of friction.

Keywords: friction model; single asperity; surface deformation; adhesion
\end{abstract}

\section{Introduction}

Due to the significant role it plays in day-to-day life, dry sliding friction has been studied for a long time, dating back to the ancient Greek time [3]-[5]. In 1616, Amonton [8] proposed that the force of friction is directly proportional to the applied load, while independent of the apparent area of contact. This empirical law was considered valid and is still applied widely till today. However, the pursuits of a deeper perception of dry sliding friction have never ended. Numerous models have been proposed to understand the mechanisms of sliding friction. In 1950s, Bowden and Tabor [14] states that the frictional force arises from two main mechanisms: the force to supply the energy of deformation and the force needed to shear the adhered junctions. Since then, both mechanisms have been modelled extensively via various approaches, as summarized below. 
Friction contributed by surface deformation have been investigated on different scales. At an atomic level, molecular dynamics (MD) are employed extensively. MD allows all atoms inside the calculation domain to move simultaneously, and the interactions between these atoms are calculated by Newton's law. Position information of all atoms at a given time is therefore trackable, which makes MD particularly suitable for solving contact mechanics on the nanoscale [27]-[32]. However, the computational costs of MD in larger size and time scale are unacceptable and limit the application of MD. In the more practical single asperity level, the finite element method (FEM) is a common approach. Plenty of works [33][36] have been carried out to investigate the friction generated by elasto-plastic deformation during single asperity sliding. However, it should be noted that performing FEM contact analysis is time consuming since a very fine mesh is needed in contact areas to ensure convergence and accurate results, while the domain needs to be large enough to avoid edge effects. In addition, the contact condition itself (no penetration of the two bodies) is not inherently incorporated in the FEM method and requires additional degrees of freedom (DOF) for a solution to be reached, which could cause unreliable results in certain scenarios. Alternatively, the semi-analytical method (SAM) provides a new approach to solve elastic and elastoplastic contact more efficiently. Various problems including single and layered material contact have been solved by SAM [37]-[40]. Also, techniques have been developed [41] to accelerate the calculation and improve the accuracy, which extend the application of SAM.

Bowden and Tabor [14] reported that adhesion affects the friction force independent of the deformation of surface asperities. The adhesion originates from a chemical bond between two surfaces. To allow relative sliding, energy input is needed for debonding, and dissipated as (part of) friction. The strength and number of bonds to be broken are controlled by shear strength and real contact area, respectively. As an experimental approaches, a surface force apparatus (SFA) has been employed for decades [42]-[45] to explore the adhesion and friction, with the advantage that contact areas can be directly measured. However, the lateral spatial resolution of SFA is rather limited [46]. In this context, modelling approaches were considered by researchers in the light of their ability to calculate real contact areas. Multiple modelling techniques have been employed. MD dominates the nanoscale friction simulation, and contact area can be calculated in straightforward ways in single asperity contact. But due to the limitations of MD on domain size, multi asperity calculation is not feasible. In this regime, a statistical model can only provide non-deterministic evaluations; FEM can perform a real contact area calculation but 
the computational costs are usually very high. SAM, however, can evaluate real contact area efficiently [41][47][48].

In this paper, a sliding dry friction model was established to calculate the friction of a pin-on-disc tribological system. Both the contributions of both the plastic deformation of asperities and the surface adhesion were considered and evaluated. A semi-analytical method (SAM) based single asperity sliding model was used to evaluate the friction generated by plastic deformation. The real contact areas were calculated by a rough surface contact model. Finally, the modelling outputs were compared with the experimental results from a pin-on-disc test-rig.

\section{Theory and Methodology}

In this work, dry sliding friction was modelled considering two contributors: elastoplastic deformation of surfaces and adhesion. See Eq. (1).

$$
F=F_{d}+F_{a}
$$

where $\boldsymbol{F}$ is the total friction, $\boldsymbol{F}_{\boldsymbol{d}}$ is the friction caused by surface plastic deformation, $\boldsymbol{F}_{\boldsymbol{a}}$ is the friction caused by adhesion.

To model the total friction $\boldsymbol{F}$, firstly a pin-on-disk sliding contact tribo-system was setup and measured at different temperatures to evaluate the total friction levels, as well as to provide surface profile input for modelling. This experimental work is described in Section 2.1. Afterwards, $\boldsymbol{F}_{\boldsymbol{d}}$ is calculated by a SAM based single asperity model, which is briefly introduced in Section 2.2. In Section 2.3, $\boldsymbol{F}_{\boldsymbol{a}}$ is evaluated based on shear strength and real contact area. The calculation of real contact area is elaborated.

\subsection{Pin-on-disc test}

Metal to metal sliding contacts at temperature of 80 and $150{ }^{\circ} \mathrm{C}$ were evaluated by a pin-on-disc tribometer, CSM HT 3-110 (CSM Instruments, Switzerland). The samples used are shown in Fig. 1. The steel ball was $10 \mathrm{~mm}$ in diameter and made of AISI 52100 with a hardness of 62-66 HRC. The centre-line average roughness $R_{a}$ is 20 $\mathrm{nm}$. The disc is of the same material as the ball (AISI 52100), with a centre-line 
average roughness $R_{a}$ of $130 \mathrm{~nm}$. The chemical compositions of AISI 52100 can be found in Tab. 1.

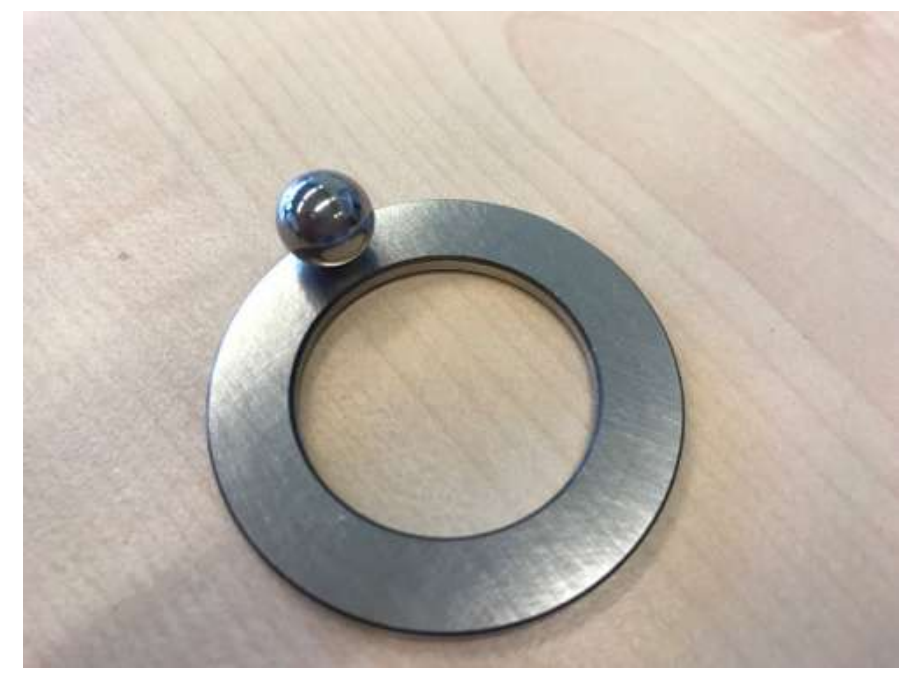

Fig. 1. Ball and disc samples used in the pin-on-disc test.

Tab. 1. Chemical composition of AISI 52100.

\begin{tabular}{cc}
\hline Element & Content (\%) \\
\hline $\mathrm{C}$ & $0.980-1.10$ \\
$\mathrm{Cr}$ & $1.30-1.60$ \\
$\mathrm{Fe}$ & $96.5-97.32$ \\
$\mathrm{Mn}$ & $0.250-0.450$ \\
$\mathrm{P}$ & $\leq 0.0250$ \\
$\mathrm{~S}$ & $\leq 0.0250$ \\
$\mathrm{Si}$ & $0.150-0.300$ \\
\hline
\end{tabular}

Both the ball and the disc were ultrasonically cleaned by 2-propanol for 10 min prior to the test. After the ball and disc were assembled in the test rig, $10 \mathrm{~mL}$ fully formulated oil Shell NS-3 was applied and the temperature was raised to the set value without applying any load. After the system reached the test temperature, the normal load was then applied and the test started. Friction forces were recorded for 12 hours, while the initial running-in periods were excluded. The detailed tests conditions are listed in Tab. 2. Sliding velocity was set slow enough $(0.1 \mathrm{~m} / \mathrm{s})$ to avoid 
hydrodynamic effects. The lateral forces and coefficient of friction data were recorded automatically during the test by the friction sensor at a rate of $5 \mathrm{~Hz}$. The tests were repeated three times for each condition.

Tab. 2. Running conditions of the pin-on-disc test.

\begin{tabular}{cc}
\hline Parameter & Value \\
\hline Load $(\mathrm{N})$ & $2,5,10$ \\
Max. Hertzian pressure $(\mathrm{GPa})$ & $0.59,0.80,1.01$ \\
Avg. Hertzian pressure $(\mathrm{GPa})$ & $0.39,0.53,0.67$ \\
Hertzian contact diameter $(\mu \mathrm{m})$ & 138 \\
Sliding velocity $(\mathrm{m} / \mathrm{s})$ & 0.1 \\
Oil temperature $\left({ }^{\circ} \mathrm{C}\right)$ & 80 and 150 \\
Duration $(\mathrm{h})$ & 12 \\
\hline
\end{tabular}

The surface profiles were measured by a confocal microscope SENSOFAR S Neox. The measuring domains are originally $264.2 \mu \mathrm{m} * 264.2 \mu \mathrm{m}$, with grid size $2048 * 2048$. The surface profiles are further used as the inputs for modelling.

\subsection{Single asperity sliding model}

In this part, a single asperity sliding model is briefly introduced. Firstly, the concept of "asperity" is firstly defined, followed by the brief introduction of the single asperity model itself.

(1) Asperity recognition and quantification

An asperity is defined as a point with a local surface height greater than its neighbouring points. Variety of definitions, including five-point summits and ninepoint summits can be used to characterize the surface asperities. In this work, the nine-point definition is adapted, to minimize the probability of detecting false summit [49]. The definition can be expressed as:

$$
z_{x, y}>z_{x-1, y-1}, z_{x-1, y}, z_{x-1, y+1}, z_{x, y-1}, z_{x, y+1}, z_{x+1, y-1}, z_{x+1, y}, z_{x, y+1}
$$

With the summit points decided, the summit radius $\boldsymbol{\beta}_{\boldsymbol{i}}$ in $\mathrm{x}$ and $\mathrm{y}$ directions are calculated by quadratic interpolation: 


$$
\begin{aligned}
& \beta_{x, i}=\frac{r_{x}^{2}}{z_{x-1, y}-2 z_{x, y}+z_{x+1, y}} \\
& \beta_{y, i}=\frac{r_{y}^{2}}{z_{x, y-1}-2 z_{x, y}+z_{x, y+1}}
\end{aligned}
$$

where $\boldsymbol{z}_{\boldsymbol{x}, \boldsymbol{y}}$ is the local surface height at the summit location $(\mathrm{x}, \mathrm{y})$, and $\boldsymbol{r}_{\boldsymbol{x}}, \boldsymbol{r}_{\boldsymbol{y}}$ denote the interferometer pixel size in $\mathrm{x}$ and $\mathrm{y}$ direction respectively.

(2) Single asperity sliding

To quantify the tangential force caused by plastic deformation during single asperity sliding, a SAM based method is employed. The schematic of contacting asperities is shown in Fig. 2. The sliding asperity contact was idealized as a normal contact between two hemispheres, following the work of Boucly [50]. The two hemispheres with radius of $\boldsymbol{R}_{\mathbf{1}}$ and $\boldsymbol{R}_{\mathbf{2}}$ are aligned with the distance between two basis planes being held constant as $\boldsymbol{h}$, while the interference depth is $\boldsymbol{d}_{\mathbf{0}}$. During the sliding, the upper hemisphere moves, while the lower hemisphere remains still. Contact force $\boldsymbol{F}_{\text {contact }}$ will be built during this process. This force can be split into horizontal and vertical components $\boldsymbol{F}_{\boldsymbol{x}}$ and $\boldsymbol{F}_{\boldsymbol{z}}$, which can be regarded as the frictional force and the normal force, respectively.

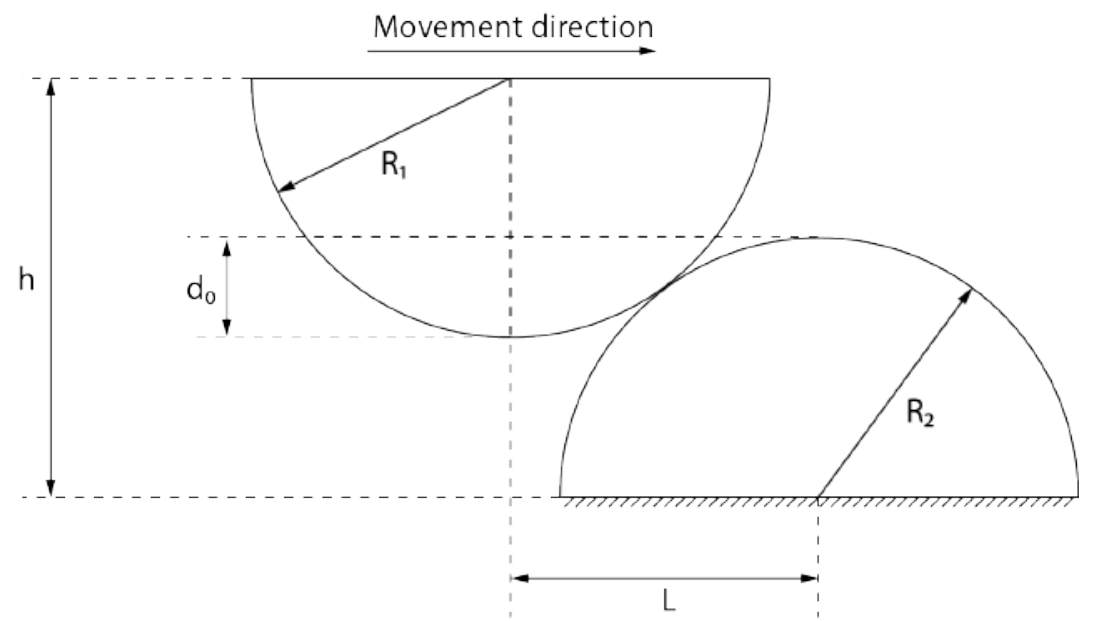

Fig. 2. Schematic of single asperity contact setup. 
To calculate $\boldsymbol{F}_{\boldsymbol{x}}$ and $\boldsymbol{F}_{\boldsymbol{Z}}$ during the whole sliding process, a discretization approach is employed. The whole sliding contact process is discretized into $\mathrm{n}$ equally distributed incremental steps. In the $\mathrm{i}^{\text {th }}$ step (see Fig.3), observe the triangle $\boldsymbol{C}_{\mathbf{2} \text {,original }}-\boldsymbol{C}_{\boldsymbol{1}}-$ $\boldsymbol{C}_{2}, \boldsymbol{d}$ is the sliding distance of the upper asperity from its original position, and $\boldsymbol{C}_{\mathbf{1}} \boldsymbol{C}_{\mathbf{2}}$ is the distance between the two centers of the asperities, which can be calculated easily by the law of Cosines. After that, the interference depth can be calculated as:

$$
\delta_{i}=R_{1}+R_{2}-C_{1} C_{2, i}
$$

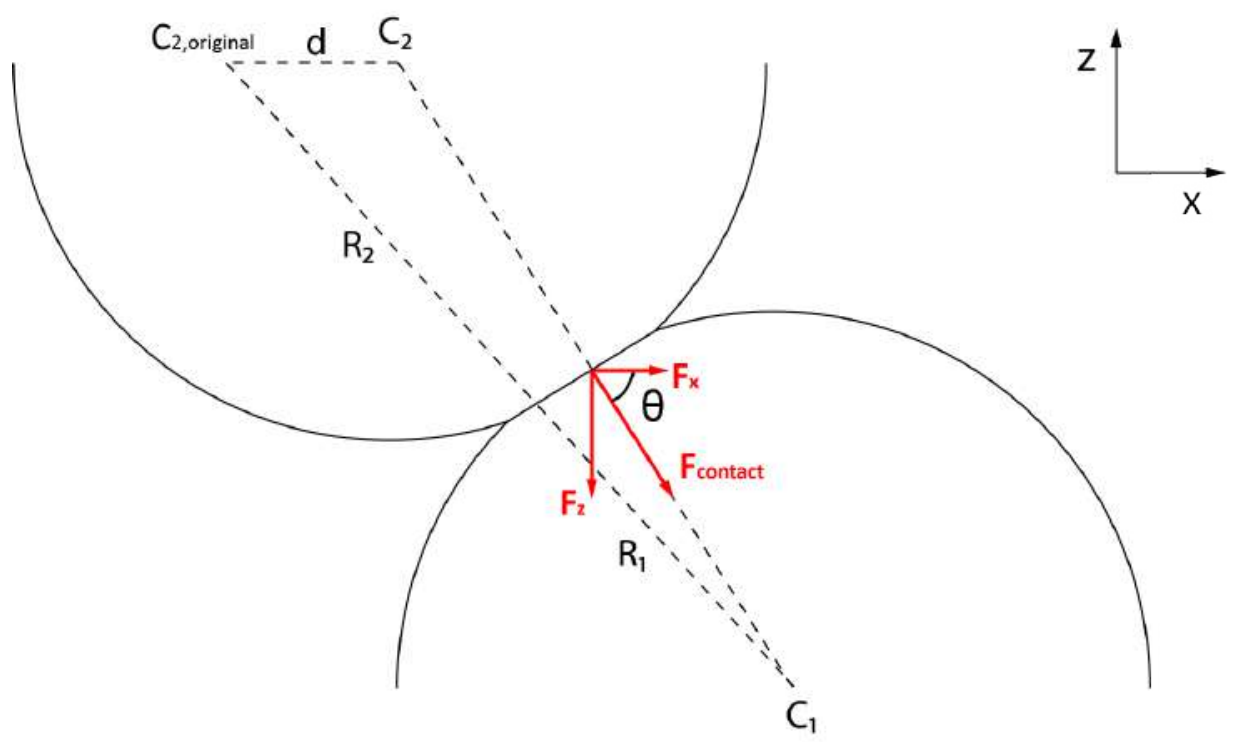

Fig. 3. Discretization of the asperity contact process.

With $\boldsymbol{\delta}_{\boldsymbol{i}}$ calculated, the contact force in each step $\boldsymbol{p}_{\text {contact }, i}$ can be calculated by a SAM based displacement-driven contact model elaborated in literature [51], which is briefly summarized in Appendix 1. The frictional force and normal force in each step then can be further calculated as:

$$
\boldsymbol{F}_{x, i}=\sum \boldsymbol{p}_{\boldsymbol{i}} * \cos \theta
$$




$$
F_{z, i}=\sum p_{i} * \sin \theta
$$

where $\theta$ is the angle between $\boldsymbol{F}_{\text {contact }}$ and the horizontal plane.

If the two asperities are not perfectly aligned as shown in Fig. 3, however, a side force $\boldsymbol{F}_{\boldsymbol{y}}$ that perpendicular to the sliding direction will be generated, while $\boldsymbol{F}_{\boldsymbol{z}}$ will not be affected. Assuming the alignment angle is $\gamma$ (ranging from $-\pi / 2$ to $\pi / 2$, while $\gamma=0$ indicates perfect alignment), the new $\boldsymbol{F}_{\boldsymbol{x}}$ and $\boldsymbol{F}_{\boldsymbol{y}}$ would be:

$$
\begin{aligned}
& \boldsymbol{F}_{x}=\cos \gamma * \boldsymbol{F}_{x, \gamma=0} \\
& \boldsymbol{F}_{y}=\sin \gamma * \boldsymbol{F}_{x, \gamma=0}
\end{aligned}
$$

Considering the global average of all the asperities, average force $\overline{\boldsymbol{F}_{\boldsymbol{x}}}$ and $\overline{\boldsymbol{F}_{\boldsymbol{y}}}$ can be calculated by integration:

$$
\begin{gathered}
\overline{F_{x}}=F_{x, \gamma=0} * \int_{-\frac{\pi}{2}}^{\frac{\pi}{2}} \cos \gamma=\frac{2}{\pi} * F_{x, \gamma=0} \\
\overline{F_{y}}=F_{x, \gamma=0} * \int_{-\frac{\pi}{2}}^{\frac{\pi}{2}} \sin \gamma=0
\end{gathered}
$$

This indicates that asperity misalignment will lead to a penalty of $2 / \pi$ on the global average tangential force in sliding direction, while in the direction perpendicular to the sliding direction, the total impact is zero.

For the whole sliding process, the coefficient of friction (COF) is defined as: 


$$
C O F=\sum_{i=1}^{n} \overline{F_{x, l}} / \sum_{i=1}^{n} F_{z, i}
$$

2.3. Calculation of friction caused by adhesion

The friction caused by adhesion is evaluated by

$$
\boldsymbol{F}_{\boldsymbol{a}}=\boldsymbol{\tau} * \boldsymbol{A}_{\boldsymbol{r}}
$$

where $\boldsymbol{\tau}$ is the shear strength of the surface film. The value is taken from literature [52]. $\boldsymbol{A}_{\boldsymbol{r}}$ is the real contact area, which is evaluated numerically by SAM in this work. The process is briefly described below.

First, the whole domain including both the contact and non-contact area, is firstly discretized into $m^{*} n$ grids, where each grid has a size of $d x^{*} d y$ (Fig. 4).

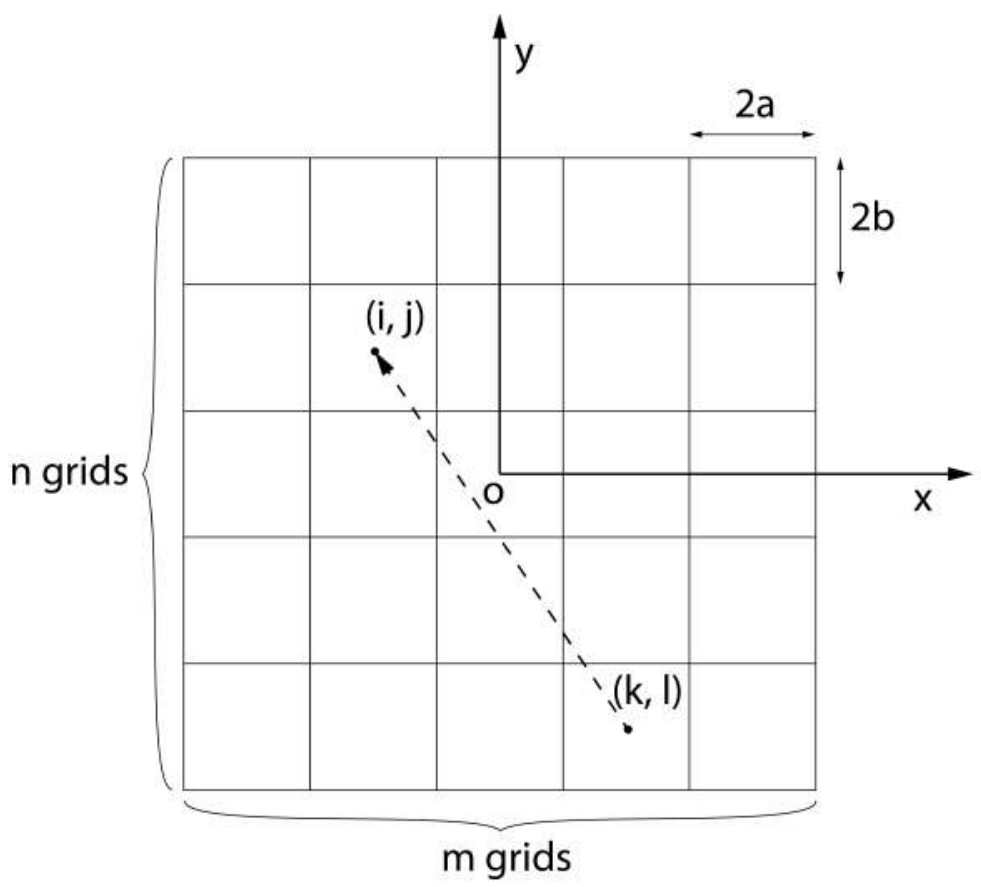

Fig. 4. Schematic of domain discretization. 
The geometry equations hold (Eq. (A.1) and (A.2) in Appendix A) and can be further re-written in discretized manner:

$\forall(\boldsymbol{i}, \boldsymbol{j}) \in \boldsymbol{I}$,

$$
u_{i j}+h_{i j}=\delta_{z} \quad(i, j) \in I_{c}
$$

and

$$
u_{i j}+h_{i j}>\delta_{z} \quad(i, j) \notin I_{c}
$$

where $\boldsymbol{u}_{\boldsymbol{i} \boldsymbol{j}}$ and $\boldsymbol{h}_{\boldsymbol{i}}$ are the displacement and geometry gap at location $(\boldsymbol{i}, \boldsymbol{j}), \boldsymbol{\delta}_{\boldsymbol{z}}$ is the rigid body movement, $\boldsymbol{I}$ represents the whole calculating domain and $\boldsymbol{I}_{\boldsymbol{c}}$ represents the real contact area where $\boldsymbol{p}_{\boldsymbol{i j}}>\mathbf{0}$.

This is a set of linear equations with the pressure distribution being explicitly included as unknowns, which can be efficiently solved by the conjugate gradient method proposed in literature [41].

First, a guess for the pressure distribution is made, and then the following iteration steps from (1) to (5) will be performed:

(1) update the gap:

$$
\begin{gathered}
g_{i j}=-\sum_{(i, j) \in I} u_{i j}-h_{i j} \\
\bar{g}=\frac{1}{N_{c}} \sum_{(k, l) \in I_{c}} g_{k l} \\
g_{i j}=g_{i j}-\bar{g} \quad(i, j) \in I
\end{gathered}
$$

(2) calculate the new conjugate direction: 


$$
\begin{gathered}
G=\sum_{(k, l) \in I_{c}} g_{i j}{ }^{2} \\
t_{i j}=g_{i j}+\delta\left(\frac{G}{G_{\text {old }}}\right) t_{i j} \quad(i, j) \in I_{c} \\
t_{i j}=0 \quad(i, j) \notin I_{c}
\end{gathered}
$$

(3) calculate the new descending step length:

$$
\begin{gathered}
r_{i j}=\sum_{(k, l) \in \Gamma} u\left(t_{k l}\right) \quad(i, j) \in I \\
\bar{r}=\frac{1}{N_{c}} \sum_{(k, l) \in I_{c}} r_{k l} \\
r_{i j}=r_{i j}-\bar{r} \quad(i, j) \in I \\
\tau=\frac{\sum_{(k, l) \in I_{c}} g_{i j} t_{i j}}{\sum_{(k, l) \in I_{c}} r_{i j} t_{i j}}
\end{gathered}
$$

(4) update the pressure distribution, enforce the inequalities and force balance:

$$
p_{i j}=p_{i j}-\tau * t_{i j} \quad(i, j) \in I_{c}
$$




$$
\begin{gathered}
\boldsymbol{I}_{o l}=\left\{(\boldsymbol{i}, \boldsymbol{j}) \in \boldsymbol{I}: \quad \boldsymbol{p}_{i j}=\mathbf{0}, \boldsymbol{g}_{i j}<\mathbf{0}\right\} \\
\boldsymbol{p}_{i j}=\boldsymbol{p}_{i j}-\boldsymbol{\tau} * \boldsymbol{g}_{i j} \quad(\boldsymbol{i}, \boldsymbol{j}) \in \boldsymbol{I}_{o l} \\
\boldsymbol{F}^{\prime}=\boldsymbol{d} \boldsymbol{x} * \boldsymbol{d y} * \sum_{(\boldsymbol{k}, \boldsymbol{l}) \in \boldsymbol{I}} \boldsymbol{p}_{i j} \\
\boldsymbol{p}_{i j}=\left(\frac{\boldsymbol{F}^{\prime}}{\boldsymbol{F}}\right) * \boldsymbol{p}_{i j}
\end{gathered}
$$

(5) check the relative error:

$$
\varepsilon=d x * d y * \sum_{(i, j) \in I}\left|p_{i j}-p_{i j}{ }^{o l d}\right|
$$

If $\varepsilon>\varepsilon_{0}$, where $\varepsilon_{0}$ is the preset tolerance, another iteration will be performed starting from step (1). Otherwise, the iteration stops and the current $\boldsymbol{p}_{\boldsymbol{i}}$ is the desired pressure distribution desired. The real contact area can also be acquired by counting the area where $\boldsymbol{p}_{\boldsymbol{i} j}>\mathbf{0}$.

Once the $\boldsymbol{p}_{i j}$ is acquired, the real contact area $\left(\boldsymbol{A}_{\boldsymbol{r}}\right)$ can be calculated as:

$$
A_{r}=\sum_{(i, j) \in I}\left(p_{i j}>0\right) * d x * d y
$$

Surface shear strength $\boldsymbol{\tau}$ can be determined by experiments. With both $\boldsymbol{\tau}$ and $\boldsymbol{A}_{r}$ acquired, the friction caused by adhesion can be calculated by Eq. (12).

\section{Results and Discussion}

The coefficient of friction acquired from the pin-on-disc test is listed in Tab. 3. The friction at $150^{\circ} \mathrm{C}$ is higher than at $80^{\circ} \mathrm{C}$. Fluctuations of the COF during the 
measurements were relatively small, yielding roughly 0.002 to 0.003 in standard deviations.

Tab. 3. Coefficient of friction acquired from pin-on-disc test.

\begin{tabular}{ccc}
\hline Temperature $\left({ }^{\circ} \mathrm{C}\right)$ & Normal load $(\mathrm{N})$ & Coefficient of friction \\
\hline \multirow{2}{*}{80} & 2 & $0.120 \pm 0.002$ \\
& 5 & $0.119 \pm 0.002$ \\
& 10 & $0.120 \pm 0.003$ \\
\hline \multirow{2}{*}{150} & 2 & $0.141 \pm 0.002$ \\
& 5 & $0.140 \pm 0.002$ \\
& 10 & $0.140 \pm 0.002$ \\
\hline
\end{tabular}

Optical microscopy and focused ion beam (FIB) measurements revealed that tribofilms were generated on post-test surfaces, ranging from 100 to $150 \mathrm{~nm}$ in thickness, see Fig. 5. Clearly, tribo-chemical reactions took place during the sliding tests. The mechanical properties of the tribofilms differ from the substrate steel, and will therefore affect the tribological behavior of the system. The properties of these tribofilms were measured and reported in literature [52]. The existence and effects of the tribofilms were considered in modelling.

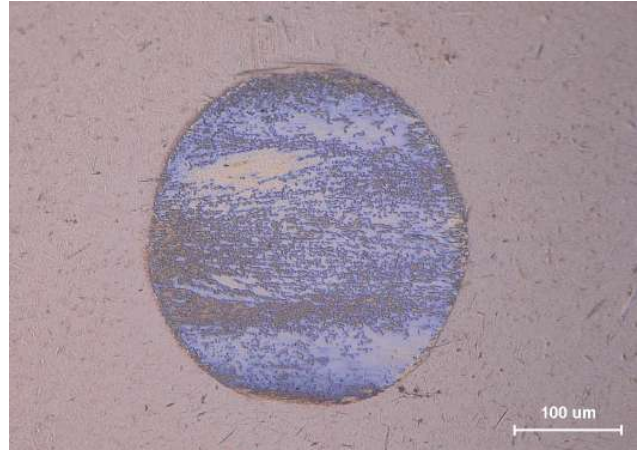

(a)

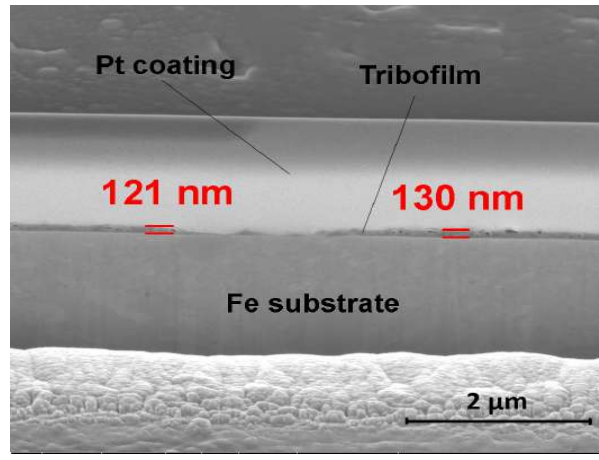

(b)

Fig. 5. Tribofilm formed on post-test surfaces: (a) optical image and (b) FIB image. 
Post-test surfaces were feed into the model as inputs. The modelling results of frictions caused by surface deformation and adhesion are summarized below in Section 3.1 and 3.2.

\subsection{Friction caused by plastic deformation}

(1) Radii of asperities

Surface profile samples of the asperities on the ball and the disc surfaces are presented in Fig. 6. The asperities on the ball surfaces are more evenly distributed with similar shapes, while the asperities on the disc surfaces vary in size and distribution.

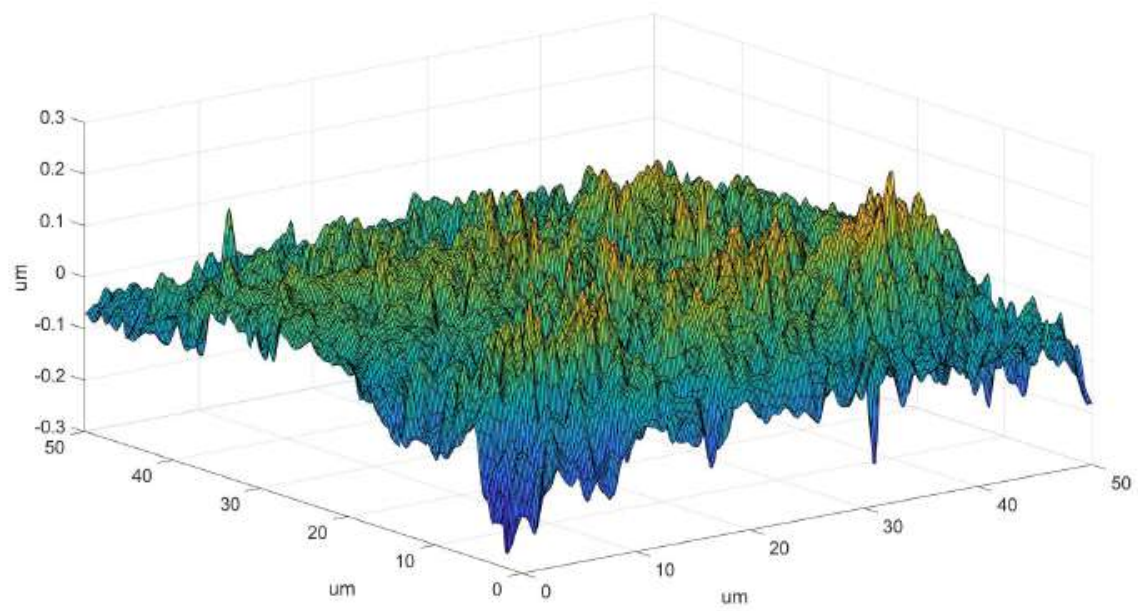

(a) 


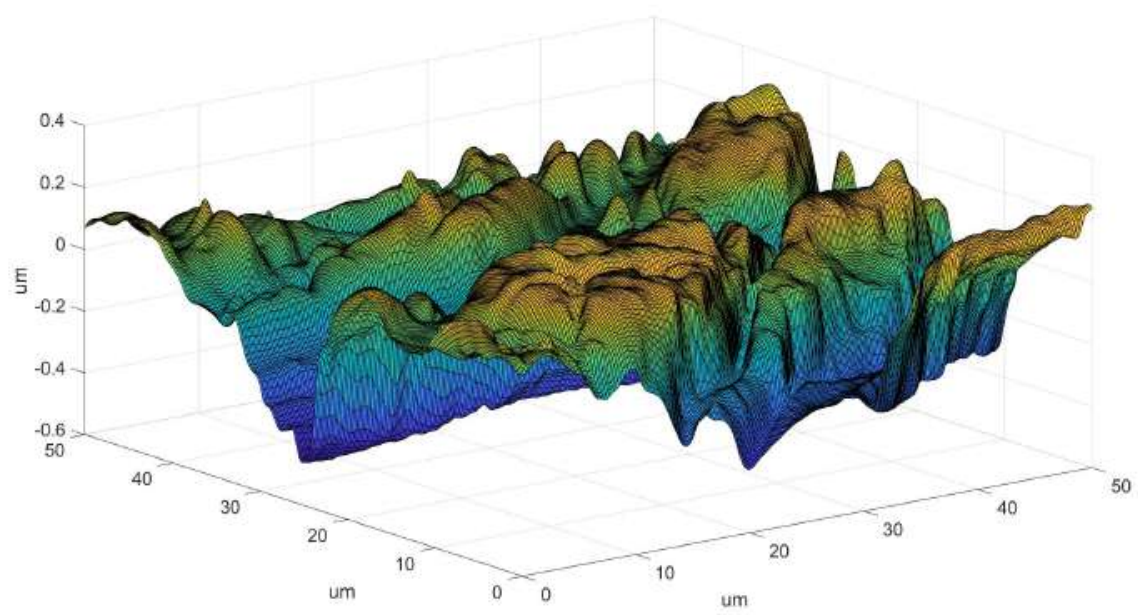

(b)

Fig. 6. Surface profiles of pin-on-disc samples (tested at $150^{\circ} \mathrm{C}$ ): (a) ball and (b) disc.

The average asperity radii of the ball and disc surfaces tested at different temperatures were calculated by Eq. (3) and (4) and given in Tab. 4. Despite the friction and wear levels are different, the asperity sizes (average radii) are comparable.

Tab. 4. Average asperity radii of ball and disc surfaces.

\begin{tabular}{ccc}
\hline \multirow{2}{*}{ Temperature $\left({ }^{\circ} \mathrm{C}\right)$} & Sample & Avg. radius $(\mu \mathrm{m})$ \\
\hline \multirow{2}{*}{80} & Disc & 6.1 \\
& Ball & 15.7 \\
\hline \multirow{2}{*}{150} & Disc & 6.6 \\
& Ball & 18.1 \\
\hline
\end{tabular}

(2) Average interference depth

In order to calculate sliding friction by using the model proposed in Section 2.2, the interference depth of asperities need to be defined, which requires mechanical 
properties of the tribofilms. AFM nano-indentations were performed to determine the elastic modulus and hardness of the tribofilms at 80 and $150^{\circ} \mathrm{C}$, as shown in Fig. 7 and 8. At 80 and $150^{\circ} \mathrm{C}$, the average elastic modulus of the tribofilms are $56 \pm 14$ and $75 \pm 16 \mathrm{GPa}$, while the average nanoindentation hardness are $2.7 \pm 0.7$ and 4.7 $\pm 1.1 \mathrm{GPa}$, respectively. Readers are referred to literature [52] for more details.

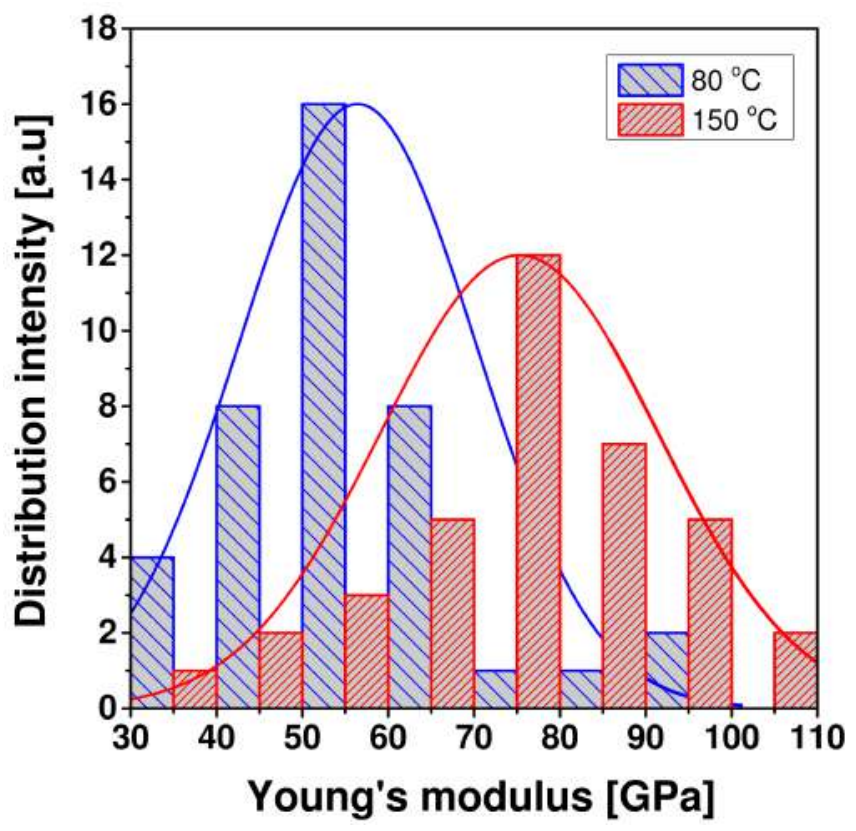

Fig. 7. Young's modulus values histogram for tribofilms formed at 80 and $150{ }^{\circ} \mathrm{C}$. The curves (fit of the normal curve to the distribution) are to guide the eye. 


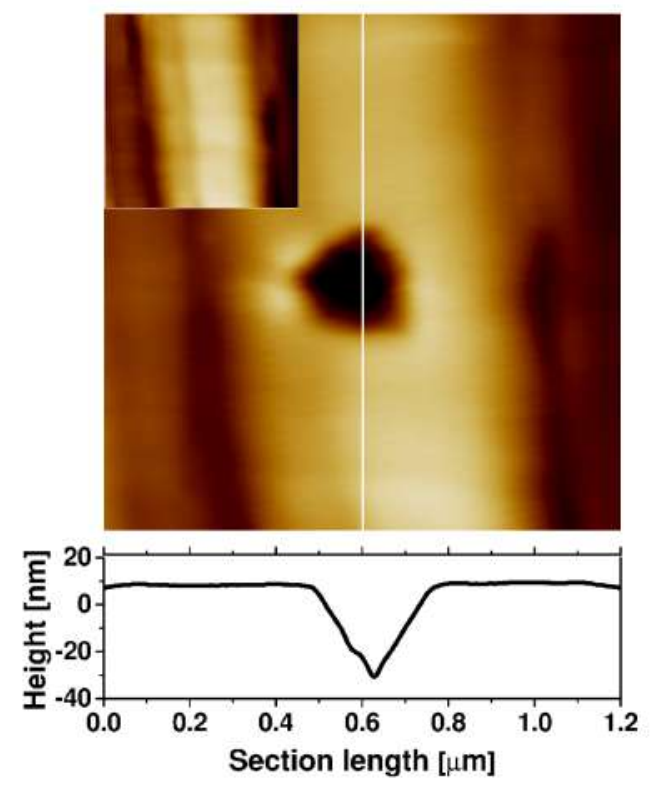

(a)

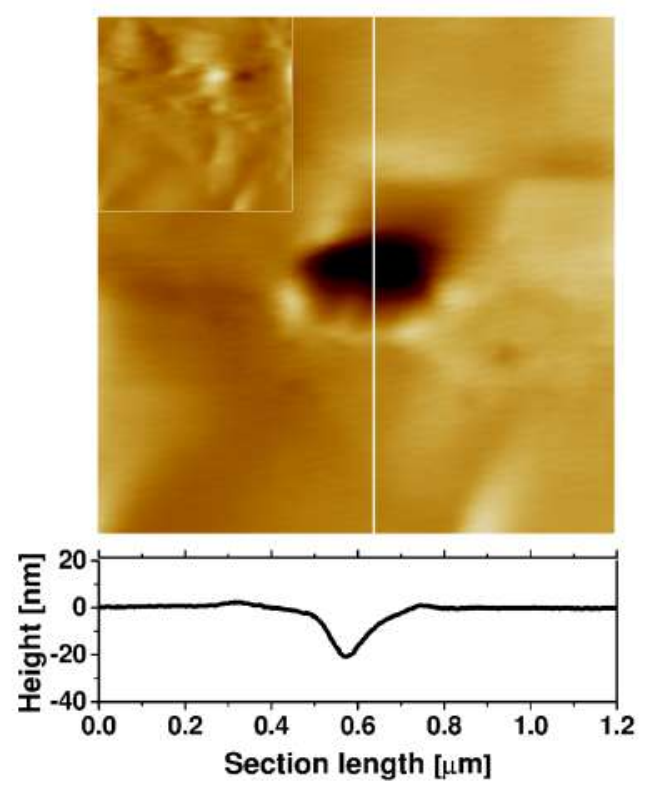

(b)

Fig. 8. AFM height images (contact mode) of the post-indented tribofilm surfaces tested at normal loads of (a) $52 \mu \mathrm{N}$ (film formed at $80^{\circ} \mathrm{C}$ ) and (b) $74 \mu \mathrm{N}$ (film formed at $150{ }^{\circ} \mathrm{C}$ ). The insets represent the investigated area before indentation. Cross-section are plotted along the section lines.

The surface deformations under different loads were calculated by the method proposed in Appendix A, and the deformation terms $\left(\boldsymbol{u}_{\boldsymbol{e}}+\boldsymbol{u}_{\boldsymbol{r}}\right)$ were taken. Results are shown in Fig. $\mathbf{9}$ and 10. It can be clearly seen that the total surface deformation consists of two parts: (1) macro-deformation which occurs globally, caused by the macro-shape of ball and flat, and (2) local micro-deformations that occur solely at the real contact areas, caused by the asperity contact. 


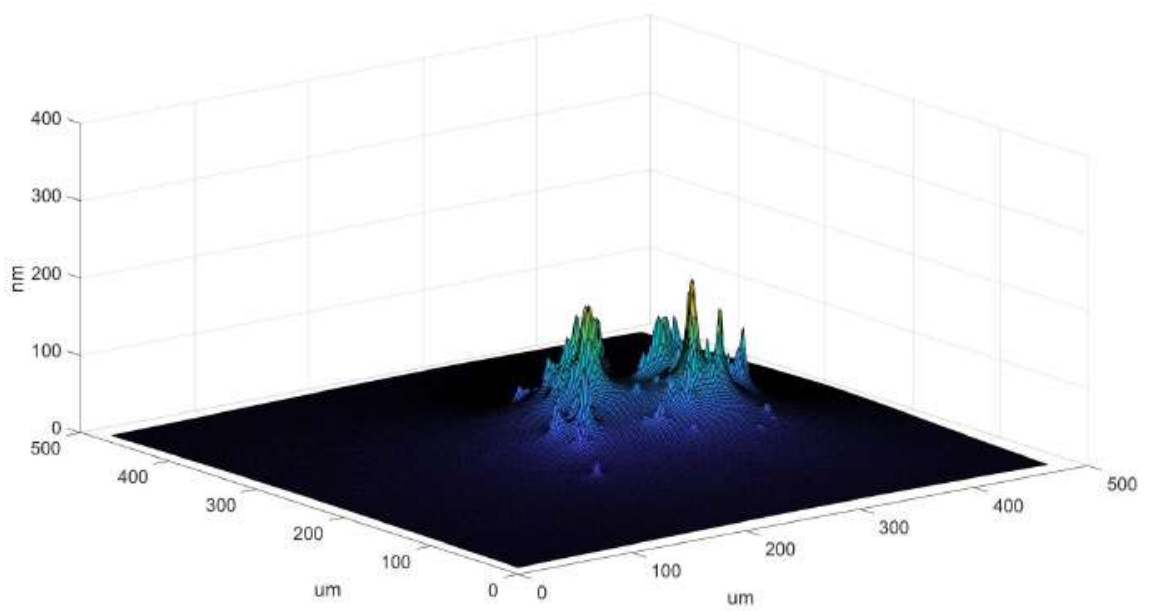

(a)

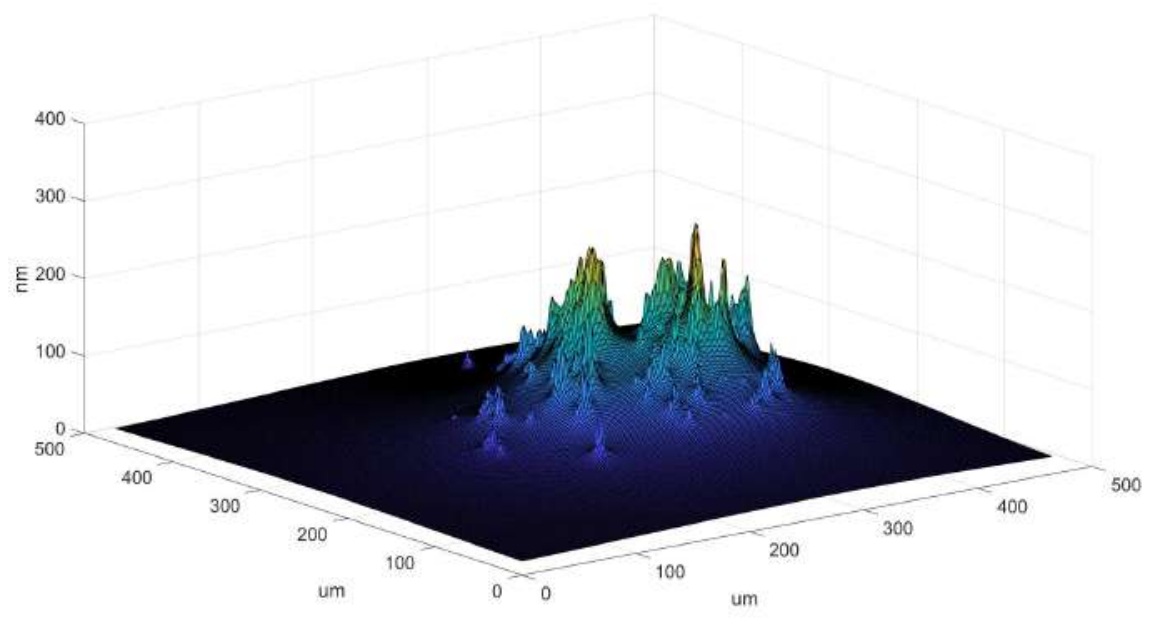

(b)

Fig. 9. Surface deformation of samples tested under different loads (both tested at $\left.150^{\circ} \mathrm{C}\right)$ : (a) $2 \mathrm{~N}$ and (b) $5 \mathrm{~N}$. 


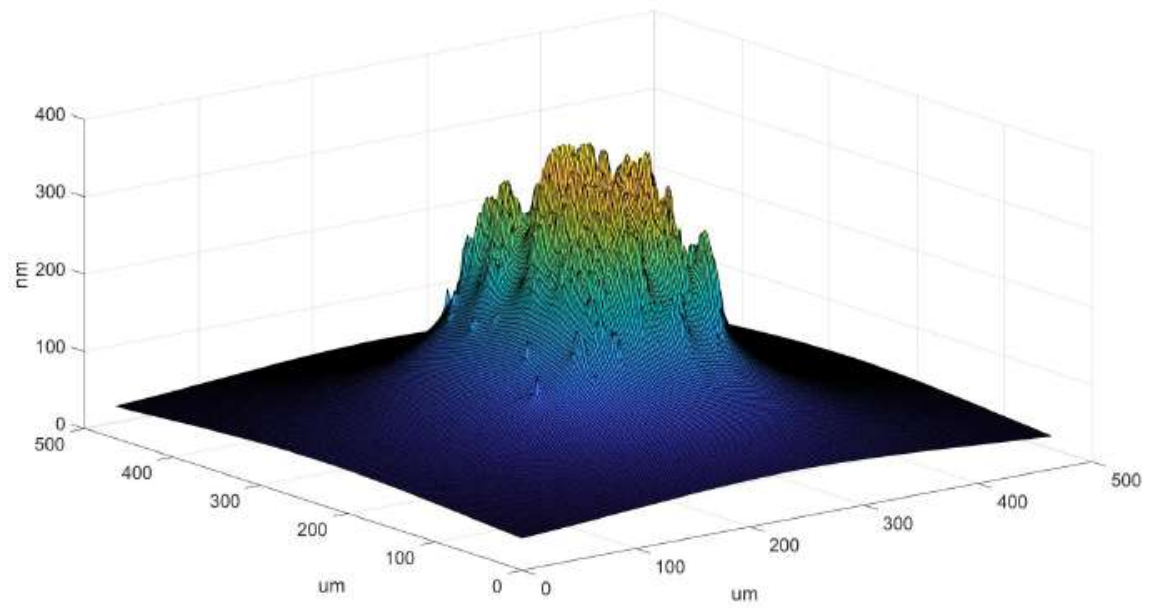

(a)

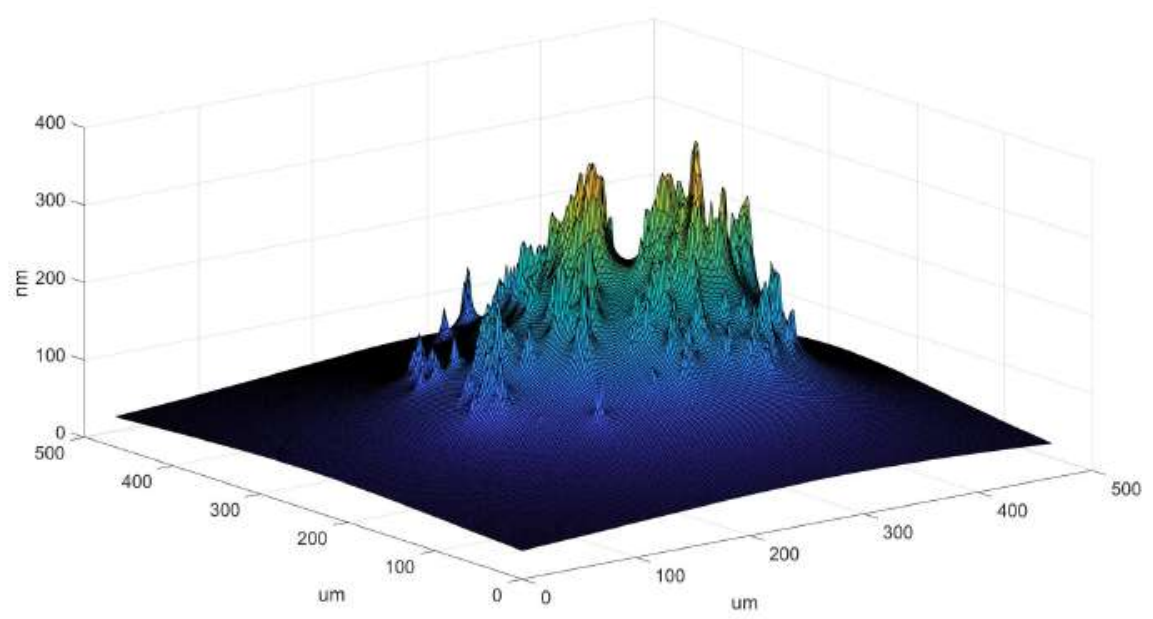

(b)

Fig. 10. Surface deformation of samples tested at different temperatures (both under $10 \mathrm{~N}$ normal load): (a) $80^{\circ} \mathrm{C}$ and (b) $150^{\circ} \mathrm{C}$.

From Fig. 9 and $\mathbf{1 0}$ it appears that the normal load affects the macro-deformation more than the micro-deformation. This is due to the elasto-plastic nature of the surface. The macro-deformations are determined mainly by the elastic properties of 
the surface. The micro-deformation, however, is strongly affected by the plastic flow, due to the very high local pressure. In this case, local asperities will be plastically deformed and will alter the surface shape, and the local micro-deformation will be reduced. This means the micro-deformations will not grow linearly with the normal load due to the non-linear nature of plasticity.

For the sliding single asperity, the interference depth is related only to the local micro-deformation. Note that because the two contact surfaces undergo similar deformations, the interference depth value should be taken as twice that of the micro-deformation value of either surface. The calculated results are listed in Tab. $\mathbf{5 .}$

Tab. 5. Average interference depths of asperities.

\begin{tabular}{ccc}
\hline \multirow{2}{*}{ Temperature $\left({ }^{\circ} \mathrm{C}\right)$} & Normal load $(\mathrm{N})$ & Avg. depth $(\mathrm{nm})$ \\
\hline \multirow{2}{*}{80} & 2 & 101 \\
& 5 & 120 \\
& 10 & 144 \\
\hline \multirow{2}{*}{150} & 2 & 160 \\
& 5 & 187 \\
& 10 & 211 \\
\hline
\end{tabular}

(3) Friction forces of single asperity sliding

With the radii of the asperities and the interference depths determined, and the mechanical properties of the tribofilm known, the sliding friction forces can be calculated by the single asperity sliding model as proposed in Section $\mathbf{2 . 2}$.

Detailed coefficients of friction results were calculated and listed in Tab. 6. The results show that the asperity deformation of the samples tested at $150{ }^{\circ} \mathrm{C}$ contributes more to friction than for the $80^{\circ} \mathrm{C}$ case due to the larger interference depths. However, for both cases, the contribution of the elasto-plastic deformation of asperities on the coefficient of friction due to deformation is fairly small, in the range of approximately 0.005 to 0.015 . 
Tab. 6. Average coefficient of friction due to plastic deformation.

\begin{tabular}{ccc}
\hline \multirow{2}{*}{ Temperature $\left({ }^{\circ} \mathrm{C}\right)$} & Normal load $(\mathrm{N})$ & COF \\
\hline \multirow{2}{*}{80} & 2 & 0.003 \\
& 5 & 0.004 \\
& 10 & 0.006 \\
\hline \multirow{2}{*}{150} & 2 & 0.007 \\
& 5 & 0.008 \\
& 10 & 0.013 \\
\hline
\end{tabular}

Therefore, it is concluded that the asperity deformation accounts for only a small fraction of the total deformation. The friction contributed by shearing the interfaces is of greater interest for the cases studied more of interest.

\subsection{Friction caused by surface adhesion}

In this part, the friction caused by the adhesion force of the pin-on-disc tribosystem is considered. First, the real contact areas are calculated by the method proposed in Section 2.3. The adhesion friction forces are subsequently calculated by Eq. (12).

(1) Real contact areas of the tribo-system

The real contact area of the pin-on-disc samples were calculated by the SAM model. The surface profiles of the ball and five sites on the disc are measured by a confocal microscope; they serve as the input for the SAM model. Example results are shown in Fig. 11 and 12 (calculated with a 1024*1024 mesh).
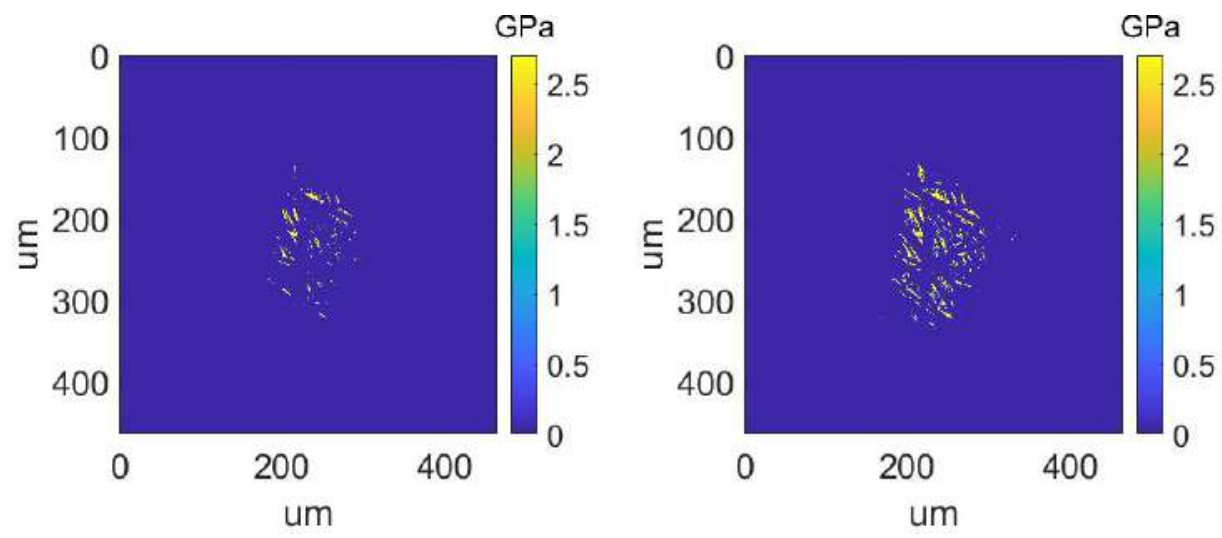
(a)

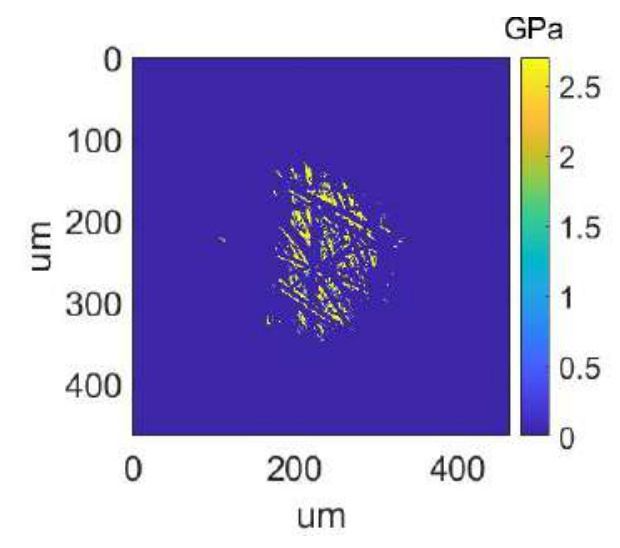

(c)

c) (b)

Fig. 11. Contact area and local pressure of samples tested at $80^{\circ} \mathrm{C}$ :

(a) $2 \mathrm{~N}$; (b) $5 \mathrm{~N}$ and (c) $10 \mathrm{~N}$.

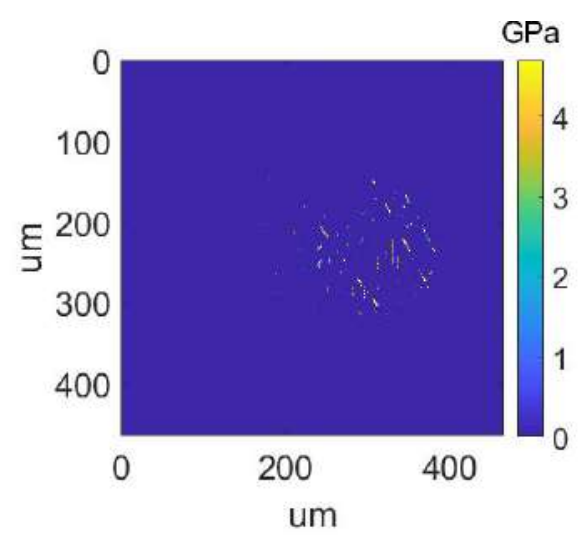

(a)

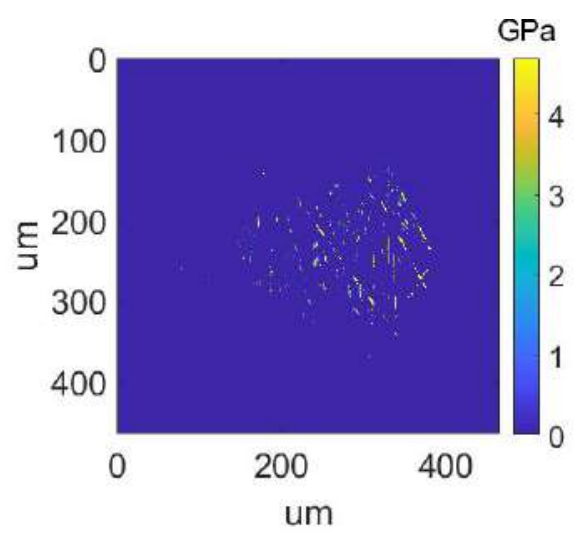

(b) 


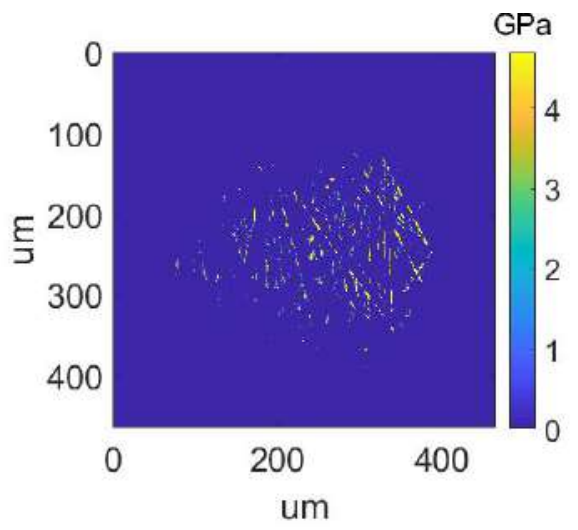

(c)

Fig. 12. Contact area and local pressure of samples tested at $150^{\circ} \mathrm{C}$ :

(a) $2 \mathrm{~N}$; (b) $5 \mathrm{~N}$ and (c) $10 \mathrm{~N}$.

The results show the contact pressures as well as the real contact areas. It can be noticed that the macro contact areas are larger for the $150^{\circ} \mathrm{C}$ case than the $80^{\circ} \mathrm{C}$ cases. This is due to the fact that more wear occurs at $150^{\circ} \mathrm{C}$, and consequently the wear scar sizes are larger. As a result, the pressure distributions of the $150^{\circ} \mathrm{C}$ cases are more disperse. This is caused by the tribofilm properties. The hardness of the tribofilm at $150{ }^{\circ} \mathrm{C}$ is greater than at $80{ }^{\circ} \mathrm{C}$, as indicated in Section 3.1. A softer tribofilm means more plastic flow. The surface shapes will be flattened more for the $80^{\circ} \mathrm{C}$ case, which leads to more continuous pressure distributions.

The friction forces were calculated by Eq. (12) and the resulting coefficients of friction are listed in Tab. 7.

Tab. 7. Average coefficient of frictions due to adhesion under different test conditions.

\begin{tabular}{ccc}
\hline Temperature $\left({ }^{\circ} \mathrm{C}\right)$ & Normal load $(\mathrm{N})$ & Coefficient of friction \\
\hline \multirow{2}{*}{80} & 2 & $0.124 \pm 0.011$ \\
& 5 & $0.131 \pm 0.015$ \\
& 10 & $0.127 \pm 0.020$ \\
\hline \multirow{2}{*}{150} & 2 & $0.152 \pm 0.011$ \\
& 5 & $0.160 \pm 0.014$
\end{tabular}


The modelling results are compared with the experiments in Fig. 13. For the $80^{\circ} \mathrm{C}$ case, the modelling results fit relatively well with the experiments; for the $150{ }^{\circ} \mathrm{C}$ case, however, noticeable differences are found. This can be attributed to the fact that the tribofilm properties are all measured at room temperature. The shear strengths of the tribofilm at $150{ }^{\circ} \mathrm{C}$ may be overestimated, leading to higher calculated friction forces.

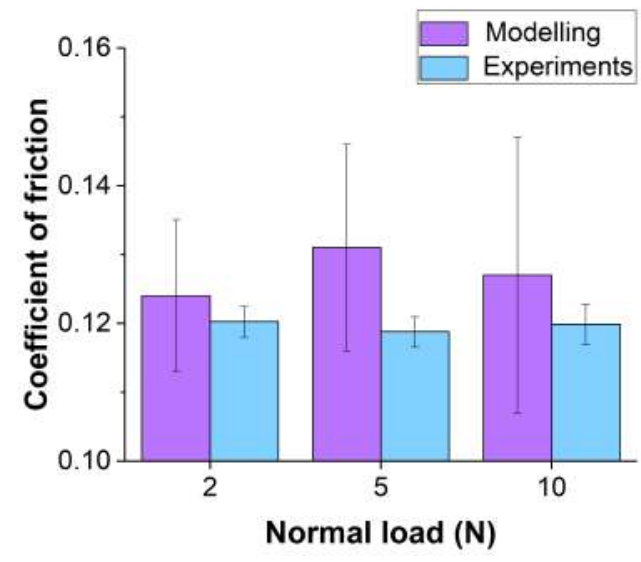

(a)

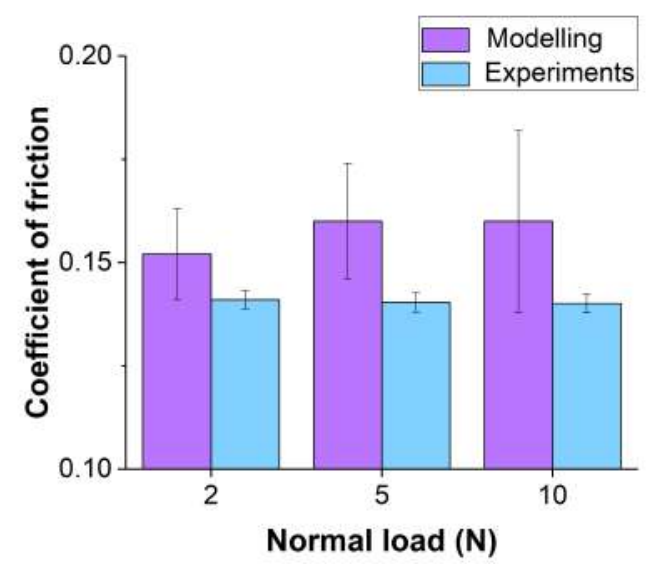

(b)

Fig. 13. Comparison of modelling and experimental results:

(a) $80^{\circ} \mathrm{C}$ and (b) $150^{\circ} \mathrm{C}$. 


\section{Conclusion}

In this paper, the friction of a pin-on-disc tribo-system is investigated. The contributions of the asperity deformation forces and the adhesion forces are calculated separately. The findings are summarized below.

(1) The radii of the asperities of ball and disc samples are approximately at the 10 $\mu \mathrm{m}$ level, while the interference depths of asperities are around the $100-200 \mathrm{~nm}$ level. The results from the single asperity sliding model shows that with these given values, the deformation forces generated account for only a small fraction of the total friction level: the deformation forces result in a coefficient of friction of around 0.003 to 0.013 level, which is only less than $10 \%$ of the total measured coefficient of friction (0.12-0.14).

(2) The adhesion forces of the pin-on-disc samples tested at 80 and $150{ }^{\circ} \mathrm{C}$ are calculated. The results show that the real contact areas of samples tested at $150^{\circ} \mathrm{C}$ are smaller and more widely distributed than in the $80^{\circ} \mathrm{C}$ case, mainly due to the greater hardness of the tribofilm. The friction calculated by the model achieve fair agreement with the experimental data.

(3) It is proposed that in the current pin-on-disc tribosystem, the main mechanism of the sliding friction is the tribofilm adhesion. The shear strength of the tribofilm together with the real contact area is dominant with respect to the sliding friction level. The deformations of asperities do not play a significant role in this tribosystem with the given test conditions. 


\section{Appendix A}

As a complement to Section 2.2, a displacement driven contact model is built in this appendix. The input of the model is the surface geometry and indentation depth, and the output is the contact pressure distribution.

(1) Setup of the contact

Elasto-plastic contact between two surfaces is illustrated in schematic form (Fig. A.1).

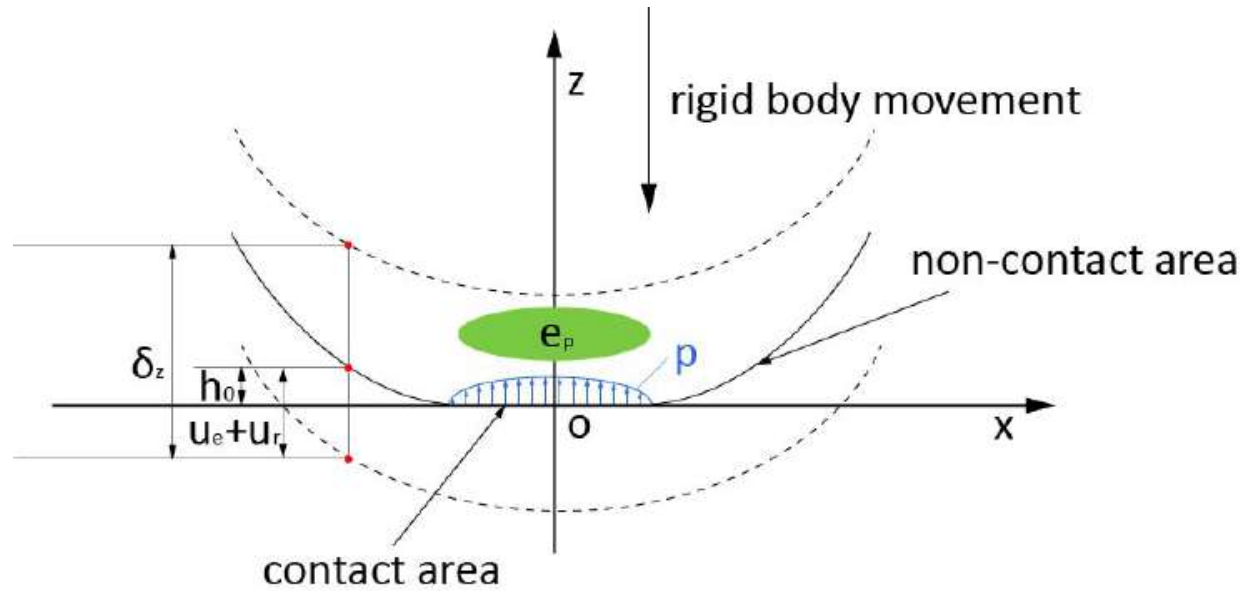

Fig A.1. Schematic of contact setup.

Geometry relations are:

$$
u_{e}+u_{r}+h_{0}=\delta_{z} \quad(p>0)
$$

and

$$
u_{e}+u_{r}+h_{0} \geq \delta_{z} \quad(p=0)
$$

where:

$\boldsymbol{p}$ is the contact pressure ( $\boldsymbol{p}>\mathbf{0}$ indicates in contact, while $\boldsymbol{p}=\mathbf{0}$ indicates out of contact), $\boldsymbol{u}_{\boldsymbol{e}}$ is the elastic displacement, $\boldsymbol{u}_{\boldsymbol{r}}$ is the plastic displacement, $\boldsymbol{h}_{\mathbf{0}}$ is the surface geometry gap and $\boldsymbol{\delta}$ is the rigid body movement, i.e. the indentation depth. 
Note that although $\boldsymbol{h}_{\mathbf{0}}$ and $\boldsymbol{\delta}_{\boldsymbol{z}}$ are known as input, $\boldsymbol{u}_{\boldsymbol{e}}$ and $\boldsymbol{u}_{\boldsymbol{r}}$ are as yet unknown yet. In reality, they are both a function of the contact pressure $\boldsymbol{p}$. In order to solve $\boldsymbol{p}$, these two functions $\left(\boldsymbol{u}_{\boldsymbol{e}}(\boldsymbol{p})\right.$ and $\left.\boldsymbol{u}_{\boldsymbol{r}}(\boldsymbol{p})\right)$ need to be defined, as given in the following section.

(2) Expression of $\boldsymbol{u}_{\boldsymbol{e}}$

By employing the Papkovich-Neuber potential and Fourier transformation, O'Sullivan and King [53] derived the frequency response of the elastic displacement and stress fields in a layered material:

$$
\begin{gathered}
\widetilde{u}_{i}^{(k)}=\frac{1}{2 G_{k}} F T\left[\varphi_{, i}^{(k)}+x \psi_{1, i}^{(k)}+z_{k} \psi_{3, i}^{(k)}-\left(3-4 v_{k}\right) \psi_{i}^{(k)}\right] \quad(A .3) \\
\widetilde{\sigma}_{i}^{(k)}=F T\left[\varphi_{, i j}^{(k)}-2 v_{k}\left(\psi_{1,1}^{(k)}+\psi_{3,3}^{(k)}\right) \delta_{i j}-\left(1-2 v_{k}\right)\left(\psi_{i, j}^{(k)}+\psi_{j, i}^{(k)}\right)+x \psi_{1, i j}^{(k)}+z_{k} \psi_{3, i j}^{(k)}\right]
\end{gathered}
$$

while

$$
\begin{aligned}
& \widetilde{\varphi}^{(k)}=A^{(k)} e^{-\alpha z_{k}}+\bar{A}^{(k)} e^{\alpha z_{k}} \\
& \widetilde{\psi}_{1}^{(k)}=B^{(k)} e^{-\alpha z_{k}}+\bar{B}^{(k)} e^{\alpha z_{k}} \\
& \widetilde{\psi}_{3}^{(k)}=C^{(k)} e^{-\alpha z_{k}}+\bar{C}^{(k)} e^{\alpha z_{k}}
\end{aligned}
$$

where $\mathrm{k}=1$ indicates coating material and $\mathrm{k}=2$ indicates substrate material, the tilde sign on top of variables stands for 2D Fourier transformation. $\boldsymbol{A}, \boldsymbol{B}$, and $\boldsymbol{C}$ are functions of surface tractions (both normal and tangential). Readers are referred to literature [53] for the detailed expressions.

Eq. (A.3) and (A.4) are essentially $\widetilde{\boldsymbol{u}_{\boldsymbol{e}}}(\boldsymbol{p})$ and $\widetilde{\boldsymbol{\sigma}}(\boldsymbol{p})$. Once these frequency responses are acquired, the displacement and subsurface stress can be calculated by DC-FFT:

$$
u_{e}=\operatorname{IFFT}\left(\widetilde{u_{z}}(p)\right)
$$

and

$$
\boldsymbol{\sigma}=\boldsymbol{I F F T}(\tilde{\boldsymbol{\sigma}}(\boldsymbol{p}))
$$




\section{(3) Expression of $\boldsymbol{u}_{r}$}

When plasticity comes into play, subsurface stress $\boldsymbol{\sigma}$ caused by contact pressure $\boldsymbol{p}$ will lead to plastic strain $\boldsymbol{e}_{\boldsymbol{p}}$, and will generate plastic displacement $\boldsymbol{u}_{r}$. The link between $\boldsymbol{\sigma}$ and $\boldsymbol{p}$ is already built by Eq. (A.6). Next, $\boldsymbol{e}_{\boldsymbol{p}}$ caused by $\boldsymbol{\sigma}$ shall be defined. In this work, the plasticity in the layered structure is considered to follow the J2 flow theory [54] and solved by the return mapping loop in Fig. A.2.

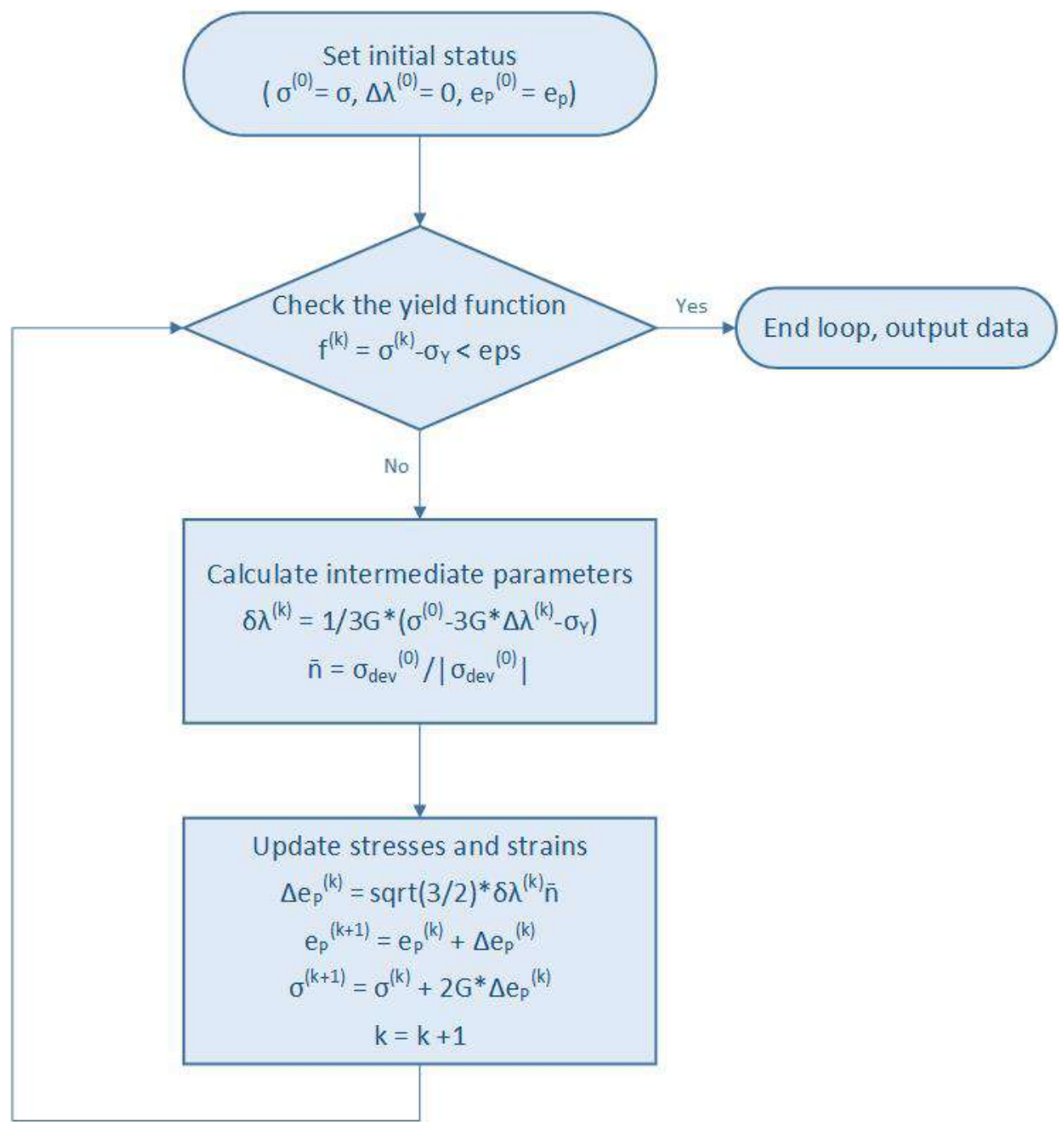

Fig A.2. Return mapping loop for calculating plastic strains. 
Next, the connection between plastic displacement and plastic strains is needed, i.e. the function of $\boldsymbol{u}_{\boldsymbol{r}}\left(\boldsymbol{e}_{\boldsymbol{p}}\right)$. Wang et. al. proposed a theory to calculate plastic displacement fields caused by plastic strains in [39]. Readers are referred to this literature for the details of solving $\boldsymbol{u}_{\boldsymbol{r}}\left(\boldsymbol{e}_{\boldsymbol{p}}\right)$ numerically.

\section{(4) Solving technique of the model}

With $\boldsymbol{u}_{\boldsymbol{e}}$ and $\boldsymbol{u}_{\boldsymbol{r}}$ being defined, the pressure distribution $\boldsymbol{p}$ is now linked to the inputs (rigid body movement $\boldsymbol{\delta}_{\boldsymbol{z}}$ and initial surface geometry $\boldsymbol{h}_{\mathbf{0}}$ ) by Eq. (A.1) and (A.2), which is in essence a linear system. However, it should be noted that the subsurface stress generated by the surface contact pressure causes plastic strains. These plastic strains cause residual displacement and stresses, which in turn alter the geometry and change the surface contact pressure. In other words, $\boldsymbol{p}$ and $\boldsymbol{e}_{\boldsymbol{p}}$ are coupled. Thus, a direct solution is difficult to acquire.

Therefore, an iteration loop is proposed to address the problem. Firstly, an initial status is set. The pressure and subsurface stress are solved first, followed by the inner plastic loop (the return mapping loop in Fig. A.2) to determine plastic deformation for the current loop. Then, the residual displacement is calculated and surface geometry is updated. Finally, the pressure distribution is recalculated based on the new surface geometry. This loop will keep running till a convergence in pressure distribution is achieved (Fig. A.3). 


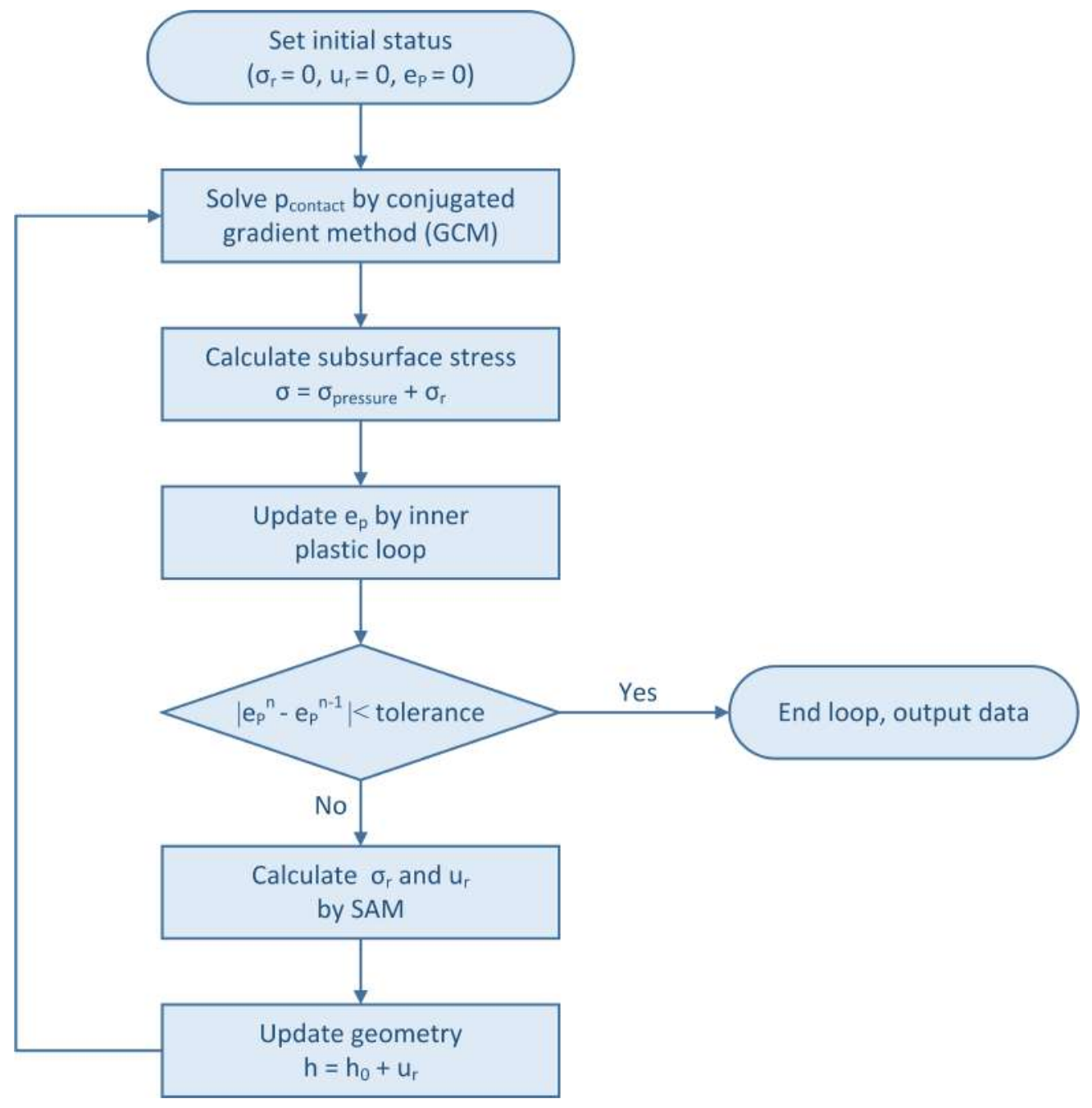

Fig A.3. Flowchart of iteration loop. 


\section{References}

[22] Chatterjee, S. (2008). Tribological properties of pseudo-elastic nickel-titanium. University of California, Los Angeles.

[23] Tipler, P. A., and Mosca, G. (2007). Physics for scientists and engineers. Macmillan.

[24] Sambursky, S. (2014). The physical world of late antiquity. Princeton University Press.

[25] Hutchings, I., and Shipway, P. (2017). Tribology: friction and wear of engineering materials. Butterworth-Heinemann.

[26] Bowden, F. P., Bowden, F. P., and Tabor, D. (2001). The friction and lubrication of solids (Vol. 1). Oxford University Press.

[27] Zhang, L. C., Johnson, K. L., and Cheong, W. C. D. (2001). A molecular dynamics study of scale effects on the friction of single-asperity contacts. Tribology Letters, 10(1), 23-28.

[28] Cha, P. R., Srolovitz, D. J., and Vanderlick, T. K. (2004). Molecular dynamics simulation of single asperity contact. Acta Materialia, 52(13), 3983-3996.

[29] Szlufarska, I., Chandross, M., and Carpick, R. W. (2008). Recent advances in single-asperity nanotribology. Journal of Physics D: Applied Physics, 41(12), 123001.

[30] Yang, C., and Persson, B. N. J. (2008). Molecular dynamics study of contact mechanics: contact area and interfacial separation from small to full contact. Physical review letters, 100(2), 024303.

[31] Anciaux, G., and Molinari, J. F. (2009). Contact mechanics at the nanoscale, a 3D multiscale approach. International journal for numerical methods in engineering, 79(9), 1041-1067.

[32] Mo, Y., Turner, K. T., and Szlufarska, I. (2009). Friction laws at the nanoscale. Nature, 457(7233), 1116-1119.

[33] Liu, G., Zhu, J., Yu, L., and Wang, Q. J. (2001). Elasto-plastic contact of rough surfaces. Tribology transactions, 44(3), 437-443.

[34] Kogut, L., and Etsion, I. (2002). Elastic-plastic contact analysis of a sphere and a rigid flat. Journal of Applied Mechanics, 69(5), 657-662.

[35] Jackson, R. L., Duvvuru, R. S., Meghani, H., and Mahajan, M. (2007). An analysis of elasto-plastic sliding spherical asperity interaction. Wear, 262(1-2), 210-219.

[36] Mulvihill, D. M., Kartal, M. E., Nowell, D., and Hills, D. A. (2011). An elasticplastic asperity interaction model for sliding friction. Tribology International, 44(12), 1679-1694.

[37] Liu, S., Wang, Q., and Liu, G. (2000). A versatile method of discrete convolution and FFT (DC-FFT) for contact analyses. Wear, 243(1-2), 101-111.

[38] Jacq, C., Ne' lias, D., Lormand, G., and Girodin, D. (2002). Development of a three-dimensional semi-analytical elastic-plastic contact code. Journal of Tribology, 124(4), 653-667. 
[39] Wang, Z., Yu, H., and Wang, Q. (2016). Analytical solutions for elastic fields caused by eigenstrains in two joined and perfectly bonded half-spaces and related problems. International Journal of Plasticity, 76, 1-28.

[40] Boucly, V., Nélias D., and Green, I. (2007). Modeling of the rolling and sliding contact between two asperities. Journal of Tribology, 129(2), 235-245.

[41] Polonsky, I. A., and Keer, L. M. (1999). A numerical method for solving rough contact problems based on the multi-level multi-summation and conjugate gradient techniques. Wear, 231(2), 206-219.

[42] Tabor, D., and Winterton, R. S. (1969). The direct measurement of normal and retarded van der Waals forces. Proceedings of the Royal Society of London. A. Mathematical and Physical Sciences, 312(1511), 435-450.

[43] Israelachvili, J. N., and Tabor, D. (1972). The measurement of van der Waals dispersion forces in the range 1.5 to $130 \mathrm{~nm}$. Proceedings of the Royal Society of London. A. Mathematical and Physical Sciences, 331(1584), 19-38.

[44] Luan, B., and Robbins, M. O. (2006). Contact of single asperities with varying adhesion: Comparing continuum mechanics to atomistic simulations. Physical Review E, 74(2), 026111.

[45] Cheng, S., and Robbins, M. O. (2010). Defining contact at the atomic scale. Tribology letters, 39(3), 329-348.

[46] Carpick, R. W., Agrait, N., Ogletree, D. F., and Salmeron, M. (1996). Measurement of interfacial shear (friction) with an ultrahigh vacuum atomic force microscope. Journal of Vacuum Science and Technology B: Microelectronics and Nanometer Structures Processing, Measurement, and Phenomena, 14(2), 1289-1295.

[47] Qazi, A., Yin, H., Sebès, M., Chollet, H., and Pozzolini, C. (2020). A semianalytical numerical method for modelling the normal wheel-rail contact. Vehicle System Dynamics, 1-19.

[48] Chen, W., Wang, Q., Huan, Z., and Luo, X. (2011). Semi-analytical viscoelastic contact modeling of polymer-based materials. Journal of Tribology, 133(4).

[49] Handzel-Powierza, Z., Klimczak, T., and Polijaniuk, A. (1992). On the experimental verification of the Greenwood-Williamson model for the contact of rough surfaces. Wear, 154(1), 115-124.

[50] Boucly, V., Nelias, D., and Green, I. Modeling of the rolling and sliding contact between two asperities. J. Tribol. 2007, 129, 235-245.

[51] Wang, C., and Schipper, D. J. (2018). On an elastoplastic sliding model for a coated single asperity. Lubricants, 6(4), 96.

[52] Wang, C., Gojzewski, H., and Schipper, D. J. (2020). A multi-technique characterization of the tribofilm formed by a fully formulated CVT fluid. Tribology international, 146, 106201.

[53] King, R. B., and O'sullivan, T. C. (1987). Sliding contact stresses in a twodimensional layered elastic half-space. International Journal of Solids and Structures, 23(5), 581-5. 
[54] Simo, J. C., and Taylor, R. L. (1985). Consistent tangent operators for rateindependent elastoplasticity. Computer methods in applied mechanics and engineering, 48(1), 101-118. 